\title{
AN INVESTIGATION OF PHYSICAL CLEANING OPERATIONS FOR FOULING ATTENUATION OF HOLLOW FIBER MEMBRANES IN DRINKING WATER TREATMENT
}

\author{
A thesis submitted to \\ the Faculty of Graduate and Postdoctoral Affairs \\ in Partial Fulfillment of the requirements for the degree \\ Master of Applied Science in Environmental Engineering
}

by

Nigel P. De Souza

(B. Eng.)

Department of Civil and Environmental Engineering Carleton University

Ottawa-Carleton Institute of Civil and Environmental Engineering

April 2012

(C2012 Nigel P. De Souza 
Library and Archives

Canada

Published Heritage

Branch

395 Wellington Street

Ottawa ON K1A ON4

Canada
Bibliothèque et

Archives Canada

Direction du

Patrimoine de l'édition

395 , rue Wellington

Ottawa ON K1A ON4

Canada
Your file Votre référence

ISBN: 978-0-494-91581-3

Our file Notre référence

ISBN: $978-0-494-91581-3$
NOTICE:

The author has granted a nonexclusive license allowing Library and Archives Canada to reproduce, publish, archive, preserve, conserve, communicate to the public by telecommunication or on the Internet, loan, distrbute and sell theses worldwide, for commercial or noncommercial purposes, in microform, paper, electronic and/or any other formats.

The author retains copyright ownership and moral rights in this thesis. Neither the thesis nor substantial extracts from it may be printed or otherwise reproduced without the author's permission.
AVIS:

L'auteur a accordé une licence non exclusive permettant à la Bibliothèque et Archives Canada de reproduire, publier, archiver, sauvegarder, conserver, transmettre au public par télécommunication ou par l'Internet, prêter, distribuer et vendre des thèses partout dans le monde, à des fins commerciales ou autres, sur support microforme, papier, électronique et/ou autres formats.

L'auteur conserve la propriété du droit d'auteur et des droits moraux qui protege cette thèse. $\mathrm{Ni}$ la thèse ni des extraits substantiels de celle-ci ne doivent être imprimés ou autrement reproduits sans son autorisation.
In compliance with the Canadian Privacy Act some supporting forms may have been removed from this thesis.

While these forms may be included in the document page count, their removal does not represent any loss of content from the thesis.
Conformément à la loi canadienne sur la protection de la vie privée, quelques formulaires secondaires ont été enlevés de cette thèse.

Bien que ces formulaires aient inclus dans la pagination, il n'y aura aucun contenu manquant. 


\section{ABSTRACT}

A number of factorial design experiments were used to investigate the combined effect of the physical cleaning operations of air scouring, backwashing and relaxation on the reduction of fouling of a submerged hollow fiber membrane ultrafiltration system treating simulated source water. The ANOVA model for a factorial design experiment as well as linear regression were used to identify important operation effects and interactions that contributed to fouling reduction. Interaction plots were used to identify and explain the antagonistic and synergistic effects of operations and interactions between operations on fouling reduction. Overall, the combination of all three cleaning operations was found to be efficient in reducing fouling. Optimal regimes for fouling reduction based on the level of filtration flux were identified. Unified as well as flux based models were also developed for the prediction of fouling over time. However, further evaluation of the efficiency of these models is required. 


\section{ACKNOWLEDGMENTS}

I would like to thank the Natural Science and Research Council (NSERC) and the Lewar Ontario Graduate Scholarship in Science and Technology (OGSST) for funding this project.

I owe my deepest gratitude to my supervisor Dr. Onita Basu. Thank you so much for the amazing opportunity and for your continuous support and guidance throughout this project. It has truly been a pleasure working with you.

I would also like to show my utmost appreciation to Dr. Marie Tudoret Chow for all her help and support in the laboratory. A big thank you also goes out to Stanley Conley, Jason Arnott and Pierre Trudel for all the technical help and good laughs.

The success of this project would not have been possible without the code writing skills of Brian Alder to whom I owe many thanks. I would also like to thank Kelly Alder for helping me save a ton of time with the data filtering macro and also for allowing Brian to stay out late to work on Operation Flux 2.0.

I would like to thank Mohammad El Resheedi for assisting me with my final experiments.

I cannot thank my family enough for their never ending love and support. Mom, Dad, Leona, Quintin and Orion. Thank you.

Finally, I would like to thank Nadia D'Lima for her incredible love and support over the years. Thank you so much for your patience and understanding. I could not have come this far without your support. 


\section{TABLE OF CONTENTS}

ABSTRACT

ACKNOWLEDGMENTS __ iii

TABLE OF CONTENTS __ iv

LIST OF TABLES ___ ix

LIST OF FIGURES __ xii

NOMENCLATURE _

CHAPTER 1 - Introduction

1.1 Rationale of the Study__ 3

1.2 Research Objectives 5

1.2.1 Preliminary Study Phase Objectives

1.2.2 Main Phase Objectives ___ 7

1.2.3 Model Development Phase ___ 8

CHAPTER 2 - Literature Review __ 9

2.1 Membrane Filtration Basics ___ 9

2.2 Membrane Filtration in Water Treatment

2.2.1 Operational Concepts ___ 13

2.2.2 UF - Membrane Configurations

2.2.3 Application of UF in Water Treatment

2.3 Membrane Fouling 20 
2.3.1 Source Water Characteristics

2.3.1.1 NOM Fouling __ 23

2.3.1.2 Inorganic Particulate Fouling __ 25

2.3.2 Membrane characteristics

2.3.3 Filtration flux and flow modes ___ 26

2.4 Fouling Attenuation 27

2.4.1 Feed Pre-treatment

2.4.2 Chemical Cleaning ___ 28

2.4.3 Physical Cleaning ___ 29

2.4.3.1 Backwashing _ 30

2.4.3.2 Relaxation $\quad 34$

2.4.3.3 Air Scouring ___ 36

CHAPTER 3 - Methodology __ 38

3.1 Materials and Methods ___ 38

3.1.1 Apparatus Setup __ 38

3.1.2 Simulated Test Water ___ 43

3.2 Experimental Design _ 45

3.2.1 Preliminary Study Phase ___ 47

3.2.1.1 Preliminary Study Phase A _ 47

3.2.1.2 Preliminary Study Phase B _ 49

3.2.2 Main Phase Experiments ___ 51

3.2.2.1 $2^{4}$ Factorial Design of Experiments ___ 51

3.2.2.2 Cleaning Operation Exclusion Tests __ 54

3.3 Cleaning Procedure

3.4 TMP/ TMP curves

3.5 Data analysis _ 57 
3.5.1 Factorial ANOVA

3.5.2 General Linear Modelling ___ 63

3.5.3 Forward Stepwise Regression

3.5.4 Prediction Model Development 67

CHAPTER 4 - Preliminary Study Phase Results _ 69

4.1 Preliminary Phase A Results

$\begin{array}{lll}\text { 4.1.1 Relaxation Duration vs. Interval } & 70\end{array}$

4.1.2 Phase A Water Quality Assessment

4.2 Preliminary Phase B Results __ 75

4.2.1 $\Delta \mathrm{TMP} \longrightarrow 76$

4.2.1.1 Relaxation vs. No Relaxation 77

4.2.1.2 Dead-end Flow Operations ___ 78

4.2.1.3 Continuous Flow Operations ___ 82

4.2.1.4 Dead-end Flow vs. Continuous Flow _ 84

4.2.2 Water Quality Assessment

4.3 Preliminary Study Phase Conclusions

CHAPTER 5 - Main Phase Experiments Results __ 92

5.1 General Observations ___ 93

5.2 Main Effects and Interactions __ 96

5.3 Flux Dependent Parameter Effects __ 106

5.3.1 Parameter Effects at a Low Filtration Flux __ 106

5.3.2 Parameter Effects at a High Filtration Flux ___ 109

5.4 Important Effects

5.5 Water Quality Assessment 
5.7 Main Phase Conclusions 122

CHAPTER 6 - Model Development Phase 124

6.1 The Original GLM 125

6.2 Time Based Prediction Model 127

6.3 Simplified Prediction Model Evaluation 134

6.4 Conclusions 137

CHAPTER 7 - Final Conclusions 139

7.1 Preliminary Phase Conclusions 140

7.2 Main Phase Conclusions 140

7.3 Model Development Phase 144

7.4 Future Studies 145

REFERENCES 146

APPENDICES 152

APPENDIX A - Zenon ZW-1 membrane specifications 153

APPENDIX B - Detailed schematics of flow lines (air, feed and permeate) 154 APPENDIX C - Operation Flux 2.0 Visual Basic Code 160 APPENDIX D - Wiring Diagram of Dual Experimental Setup 193 APPENDIX E - Trouble Shooting for Operation Flux 2.0 and WinLIN 194

APPENDIX F - Simulated Test Water Ingredients/ Organic Component Calibration Curve 199 
APPENDIX G - Macro code for filtering backwash and relaxation data points from TMP curves 200

APPENDIX H - ANOVA MODEL for a $2^{4}$ factorial design 203

APPENDIX I - Similarities between ANOVA parameters and regression coefficients 205 APPENDIX J - Water quality data from Preliminary Study Phase A 206

APPENDIX K - Water quality data from Preliminary Study Phase B 207

APPENDIX L - Main Phase Experiments: $2^{4}$ factorial design replicates 209

APPENDIX M - 4-way ANOVA model with TMP (Main Phase Experiments) 211

APPENDIX N - 3-way Interaction Plots - Main Phase Experiments 212

APPENDIX O - Stepwise Linear Regression Output using JMP 9.0 by SAS 214

APPENDIX P - Water Quality from Main Phase Low Filtration Flux experiments 215

APPENDIX Q - Water Quality from Main Phase High Filtration Flux Experiments 218

APPENDIX R - Daily Regression coefficients for 5-day $2^{4}$ factorial Main Phase Experiments 221

APPENDIX S - Regression coefficient estimation for 5 day prediction model 222 APPENDIX T - Half Normal Plot for the identification of important effects 223 


\section{LIST OF TABLES}

Table 2.1 - Characterization of membrane filters (adapted from AWWA, 2005 and Judd, 2011) 10

Table 2.2 - Summary of findings from studies investigating the effect of backwashing (BW) and air assisted backwashing on fouling. 32

Table 2.3 - Summary of findings from studies investigating the effect of relaxation on fouling 35

Table 3.1 $-2^{2}$ factorial design used in the Preliminary Study Phase to investigate the effect of relaxation duration and interval on membrane fouling. 47

Table 3.2 - Operational parameters used in Preliminary Study Phase A - Relaxation duration vs, Frequency 48

Table 3.3 - Operational parameters used in Preliminary Study Phase B - Dead-end vs. Continuous Flow Operations 50

Table $3.4-2^{4}$ factorial design used to investigate the effect of filtration flux, air flow rate, backwashing duration and relaxation duration on membrane fouling 53

Table 3.5 - Fractional factorial design used to investigate the impact of cleaning parameters exclusion on membrane fouling. Filtration flux $=38$ LMH for 15 minutes 
Table 3.6 - ANOVA model for a $2^{2}$ factorial design. 59

Table 4.1 - $\Delta$ TMP (mbar) observed for Preliminary Study Phase A experiments _ 72 Table 4.2 - Main and Interaction effects for Preliminary Study Phase A experiments 73

Table 4.3 - $\triangle$ TMP (mbar) observed for Phase B dead-end flow experiments 80

Table 4.4 - Main and interaction effects for Phase B dead-end flow experiments._ 81 Table 4.5 - $\triangle$ TMP (mbar) observed for Phase B continuous flow experiments 83

Table 4.6 - Main and interaction effects for Phase B continuous flow experiments. 83 Table 4.7 - Effects for the comparison of Continuous flow vs. Dead-end flow operations 85

Table 4.8 - Comparison of Turbidity, COD and Temperature in the reactor tank for Phase B experiments 89

Table 5.1 - TMP change and permeate volume produced at the end of 5 day long Main Phase Experiments. 94

Table 5.2 - Main effects and interactions estimated using a 4-way ANOVA model and general linear modeling. 97

Table 5.3 - Important effects identified by stepwise linear regression 115 
Table 5.4 - Water quality assessment of least (Exp. 3 and 15) and most (Exp. 2 and 6) fouling experiments.

Table 5.5 - Changes in water quality parameters noticed during cleaning operation exclusion tests 121

Table 6.1 - Comparison of fouling predicted by the Original and Reduced GLMs for each experiment and the corresponding Sum of Squares of Residuals. 127

Table 6.2 - Comparison of preliminary prediction models for the prediciton of fouling after 5 days.

Table 6.3 - Summary of important effects (regression coefficients) identified in the study.

Table 6.4 - Comparison of TMP prediction by the Simplified Prediction Model (SPM) when using three different Regression Estimation Models (REM).

Table 6.5 - Regenerated equations for the estimation of regression coefficients over time by Option 1 and 2 134 


\section{LIST OF FIGURES}

Figure 2.2 - Schematic of a) Reversible and b) Irreversible fouling 22

Figure 3. 1 - Schematic of the bench scale membrane filtration setup 39

Figure 3.2 - Research performed at the Three Study Phases 46

Figure 3.3 - Interaction plots: a) no interaction; b) antagonistic interaction; c) synergistic interaction (adapted from Kutner et al. (2005)) 61

Figure 4.1 - Fouling over time during Preliminary Study Phase A experiments 71

Figure 4.2 - Effect of duration vs. interval on $\triangle T M P$ in Preliminary Phase A experiments 73

Figure 4.3 - The effect of relaxation exclusion on fouling under continuous flow (CF) and dead-end flow (DE) operations 78

Figure 4.4 - TMP changes for Preliminary Phase B experiments employing dead-end flow operation 79

Figure 4.5 - Effect of duration vs. interval on $\triangle T M P$ in Phase B dead-end flow experiments 81

Figure 4.6 - TMP changes for Phase B experiments employing a continuous flow (80\% recovery) operation 82 
Figure 4.7 - Effect of duration vs. interval on $\triangle T M P$ in Phase B continuous flow experiments

Figure 4.8 - Interactions between a) Flow operation and relaxation duration, b)

Flow operation and relaxation interval and c) Relaxation duration and interval from Preliminary Phase B experiments.

Figure 4.9 - Comparison of TMP changes in preliminary phase B experiments 87

Figure 5.1- State of membrane surface of a) a clean membrane, b) after Exp. 2 (most fouling) and c) after Exp. 5 (least fouling) 95

Figure 5.2 - Two way interactions between Filtration flux and a) Air flow rate, b) Backwash duration and c) Relaxation duration. 99

Figure 5.3 - Two way interactions between Air flow rate and a) Filtration flux, b) Backwash duration and c) Relaxation duration. 100

Figure 5.4 - Two way interactions between Backwash duration and a) Filtration flux, b) Air flow rate and c) Relaxation duration. 101

Figure 5.5 - Two way interactions between Relaxation duration and a) Filtration flux, b) Air flow rate and c) Backwash duration. 102

Figure 5.6 - TMP change due to low filtration flux (19 LMH) and short backwash duration (10 sec.) 107

Figure 5.7 - TMP change due to low filtration flux (19 LMH) and long backwash duration (20 sec.) 108 
Figure 5.8 - Identification of the most and least efficient cleaning regimes for low filtration fux operations.

Figure 5.9 - TMP change due to high filtration flux (38 LMH) and short backwash duration (10 sec.)

Figure 5.10 - TMP change due to high filtration flux (38 LMH) and long backwash duration (20 sec.) (Note: Curves for combinations involving 15 min. relaxation durations overlap)

Figure 5.11 - Identification of the most and least efficient cleaning regimes for high filtration fux operations.

Figure 5.12 - Sum of Squares of Residuals comparison for the identification of important effects

Figure 5.13 - Effect of cleaning operation exclusion under high fouling conditions.

Figure 5.14 - Membrane surface conditions due to cleaning operation exclusion tests (From left to right - With air scouring, backwashing and relaxation (5 days);

Without relaxation (2 days); Without backwashing (2 days); Without air scouring (2 days)) 120

Figure 6.1 - Evaluation of Simplified Prediction Models for the prediction of fouling over 10 days (Flux $\rightarrow 38$ LMH). 136 


\section{NOMENCLATURE}

\begin{tabular}{|c|c|}
\hline ANOVA & Analysis of variance \\
\hline CEB & Chemically enhanced backwash \\
\hline CIP & 'Clean in place' chemical cleaning operation \\
\hline COD & Chemical oxygen demand \\
\hline FA & Fulvic acid \\
\hline GFM & Gas mass flow meter \\
\hline GLM & General linear model \\
\hline HA & Humic acid \\
\hline$\overline{\mathrm{i}, \mathrm{j}, \mathrm{k}, \mathrm{l}}$ & Levels of factors A, B, C and D respectively in a $2^{4}$ factorial design \\
\hline $\mathbf{J}$ & Filtration flux (LMH) \\
\hline LMH & Standard unit for membrane flux - Litres/meter $/$ hour \\
\hline LPM & Liters per minute \\
\hline MF & Microfiltration \\
\hline $\mathrm{m}^{\mathrm{k}}$ & General notation of a factorial design ( $\mathrm{m} \rightarrow$ levels $\& \mathrm{k} \rightarrow$ factors) \\
\hline$\overline{\mathrm{NF}}$ & Nanofiltration \\
\hline NOM & Natural organic matter \\
\hline PPM & \begin{tabular}{|l} 
Preliminary Prediction Model \\
\end{tabular} \\
\hline REM & Regression Estimation Model \\
\hline RO & Reverse Osmosis \\
\hline sMBR & Submerged membrane bioreactor \\
\hline SMP & Soluble microbial products \\
\hline SPM & Simplified Prediction Model \\
\hline SSR & Sum of Squares of Residuals \\
\hline TC, TOC & Total Carbon, Total Organic Carbon \\
\hline THMs & Trihalomethanes \\
\hline TMP, $\Delta \mathrm{TMP}_{\mathrm{day} 5}$ & Transmembrane pressure, Change in TMP after 5 days \\
\hline UF & Ultrafiltration \\
\hline$\alpha, \beta, \gamma, \sigma$ & $\begin{array}{l}\text { Main effects corresponding to factors A, B, C and D respectively in the } \\
\text { ANOVA model of a } 2^{4} \text { factorial design of experiments }\end{array}$ \\
\hline$\overline{\beta_{x}}$ & Regression coefficient of a GLM representing a $2^{4}$ factorial design \\
\hline$\mu_{\mathrm{ijk} \mathbf{1}}$ & $\begin{array}{l}\text { Mean response for a treatment combination in the ANOVA model for a } 2^{4} \\
\text { factorial design of experiments }\end{array}$ \\
\hline$\mu_{\mathrm{i} \ldots}, \mu_{\mathrm{j} . .}, \mu_{. \mathrm{k} .}, \mu_{\ldots 1}$ & $\begin{array}{l}\text { Factor level means for factors A, B, C and D respectively in the ANOVA } \\
\text { model for a } 2^{4} \text { factorial design of experiments }\end{array}$ \\
\hline
\end{tabular}




\section{CHAPTER 1 - Introduction}

The continuously increasing need for clean drinking water has resulted in the search for more economic and efficient means of treating source waters. Over the past two decades, the use of membrane filtration has emerged as a sound alternative to conventional methods of source water treatment due to the higher quality of water produced, the smaller footprint required as well as the decreasing costs of operating and maintaining membrane treatment facilities (Tian et al., 2010).

Membrane filtration is a process in which particulate matter in source water is separated from solution mainly by size exclusion. The extremely small pore sizes $(0.001$ $\mu \mathrm{m}-10 \mu \mathrm{m}$ ) of membranes aid in the provision of a high quality of water due to the effective removal of turbidity and organic matter from the feed water (Gao et al., 2011). Studies have also shown that membranes are effective in removing bacteria and viruses (Konieczny, 1998: Jacangelo et al., 2005) as well as cryptosporidium and giardia (USEPA, 2005, Gao et al., 2011) and thus provide a level of disinfection during treatment. As a result, smaller amounts of disinfection chemicals are required accompanied by a corresponding reduction of operational costs. Furthermore, the costs of membrane filtration continue to decrease over time with advancements in membrane technology as newer and cheaper membrane materials are developed (Tian et al., 2010). 
While membranes provide a higher quality of treated water along with the reduction in the use of chemicals, operation costs and facility footprint, the unavoidable shortcoming of membrane fouling is a major concern. The purpose of membrane filtration is to remove colloids and suspended particles in the source water to provide clean water for drinking purposes. However these particles contribute to the clogging of membrane pores along with the buildup of a cake layer on the membrane surface. Studies have shown that turbidity (Tian et al., 2010) as well as natural organic matter (NOM) (Sasaki et al., 2008) play a significant role in the fouling of membranes.

Fouling of membranes is extremely unfavourable as it results in an increase in the transmembrane pressure (TMP) along with a potential decrease in the productivity of the membrane (Bottino et al., 2009). A high TMP is a threat to the service life of the membrane while extra pump costs might be inferred to compensate for potential losses in membrane productivity (Guo et al., 2008). Fouling cannot be stopped, but the rate of fouling can be slowed down using various source water pre-treatment methods and membrane cleaning operations.

Pre-treatment methods have proved to be effective in decreasing membrane fouling and improving the quality of the treated water. However, the costs associated with pre-treatment are high and the expected reduction in fouling may not always be a guarantee (Kabsch-Korbutowicz et al., 2006). On the other hand, cleaning operations are fairly low in cost yet have not been given as much attention in literature (Porcelli and Judd, 2010). To help fill in some of the gaps in literature pertaining to the effects of cleaning methods on membrane fouling, this study focused on the combined effect of the 
physical cleaning methods of air scouring, backwashing and relaxation on reducing fouling in drinking water treatment.

\subsection{Rationale of the Study}

Although there has been much advancement in membrane technology over the past two decades, membrane fouling continues to be a major issue limiting the widespread applicability of membrane filtration in water treatment (Gao et al., 2011). While membrane fouling cannot be eliminated, measures can be taken to help ensure that the membranes do not reach a level of irreversible fouling at which permeability cannot be recovered. The main operational criteria to ensure maximum life and productivity of a membrane is to operate the system while maintaining the flux at or below the critical flux value thus ensuring only reversible fouling (Van der Marel et al., 2009). Wherein the critical flux is defined as the flux above which irreversible fouling requiring chemical cleaning occurs (Judd, 2011).

Since the critical flux is an indicator of the onset of irreversible fouling, a number of mathematical models also known as flux step models have been developed over the years to determine the critical flux of a membrane (Le Clech et al., 2003; Van der Marel et al., 2009). These models suited for membranes in wastewater treatment, incorporate various physical cleaning operations such as backwashing, air scouring and even relaxation to ensure only reversible fouling occurs. These models were developed mainly for the prediction of the onset of fouling and do not emphasize the effect of the various cleaning operations on the reduction of fouling (Porcelli and Judd, 2010). While 
mathematical models like those developed by Le Clech (2003) and Van der Marel (2009) might not be directly applicable to drinking water treatment due to the large differences in water quality parameters of raw water and wastewater, the physical cleaning operations employed in these studies can be extremely beneficial.

Backwashing is an operation in which permeate is made to flow in the opposite direction through the membrane. As a result, a backward force is applied on the fouling layer thus pushing away foulants from the surface of the membrane and preventing any strong attachment between the foulants and the membrane surface (Gao et al., 2011). The use of air bubbles to scour the surface of the membranes and thus displace foulants off the membrane surface has also been effective for the purpose of fouling reduction (Tian et al., 2010). Furthermore, Tian et al. (2010) found that fouling was reduced with help of relaxation in which permeate production was paused for brief time intervals during operation.

While all three physical cleaning operations have been found to be effective in reducing membrane fouling, there is a lack of literature in which the effect of the combination of the three operations has been studied. Furthermore, with the growing popularity of membranes in drinking water treatment, it is pertinent that models determining the effect of natural source water qualities and operational regimes on fouling in drinking water treatment be established. Such models are currently lacking but are essential for understanding the fouling phenomenon due to natural source water constituents. Thus, this study investigated the effect of the physical cleaning operations backwashing, air scouring and relaxation on the minimization of membrane fouling of a submerged hollow fiber membrane ultrafiltration system treating simulated source water. 
A statistical model was also developed and tested to aid in the determination of fouling of a submerged membrane system when the desired physical cleaning parameters are known.

\subsection{Research Objectives}

The main objectives of the research study were as follows:

- To investigate the combined effect of physical cleaning operations (air scouring, backwashing and relaxation) on the minimization of fouling of a bench scaled submerged hollow fiber ultrafiltration membrane system.

- To develop a statistical model based on the results of a $2^{4}$ factorial design of experiments for the estimation of fouling over time of a submerged hollow fiber ultrafiltration membrane.

In order to meet these research objectives, the study was carried out in three phases; the Preliminary Study Phase, the Main Phase Experiments and the Model Development Phase. The objectives of these phases are elaborated in the following sections.

\subsubsection{Preliminary Study Phase Objectives}

Prior to conducting the Main Phase Experiments, a number of preliminary experiments were performed to verify some of the test parameters and operating conditions to be used in the Main Phase Experiments. This preliminary phase consisted of 
two $2^{2}$ factorial design of experiments. The objectives of these preliminary experiments were as follows:

- To analyze the impact of relaxation parameters (relaxation duration vs. relaxation interval) on the fouling of a submerged hollow fiber membrane.

- To examine the effect of flow operations (dead-end vs. continuous) on the fouling of a submerged hollow fiber membrane.

Of the three physical cleaning operations investigated in this study, relaxation was found to be the operation used the least in past studies. Due to the lack of accepted values pertaining to relaxation regimes, the first $2^{2}$ preliminary factorial design experiments were performed to identify the most favorable fouling relaxation parameter to be used in the Main Phase Experiments.

The second $2^{2}$ preliminary factorial design experiments were performed to identify the most practical type of flow operation to be used in 5 day long Main Phase Experiments. The two flow operations tested were a dead-end flow operation and a continuous flow operation. In the dead-end flow operation, the feed flow and permeate flow were set to be identical resulting in an accumulation of foulants in the reactor tank over time. In the case of continuous flow operations the feed flow was set at a higher rate than the permeate flow thus allowing $80 \%$ recovery of permeate in the system as well as a continuous recycling of water in the feed tank.

A detailed explanation of the experimental design for these Preliminary Study Phase experiments can be seen in CHAPTER 3 Section 3.2.1. The results and analysis of the results of these experiments are discussed in CHAPTER 4. 


\subsubsection{Main Phase Objectives}

Taking into account the results from the Preliminary Study Phase experiments, the Main Phase Experiments were performed to fulfill the main objectives of this research project. The objectives of these experiments were as follows:

- To investigate the effect of physical cleaning operations on the reduction of membrane fouling.

- To identify possible synergistic relationships between air scouring, backwashing, relaxation and filtration flux from the results of a $2^{4}$ factorial design of experiments.

- To examine the effect of cleaning operation exclusion on fouling reduction.

The Main Phase Experiments were performed to investigate the effect of air scouring, backwashing and relaxation along with filtration flux on the fouling of a submerged hollow fiber membrane system while employing a $2^{4}$ factorial design of experiments. A fractional $2^{3}$ factorial design of experiments was also performed during this phase to examine the effect of the exclusion of individual cleaning operations from a cleaning regime involving air scouring, backwashing and relaxation on the reduction of fouling. The experimental design of the $2^{3}$ and $2^{4}$ factorial design experiments can be seen in CHAPTER 3 Section 3.2.2. The results and statistical analysis of the results including the effect of important cleaning operations and the interactions between operations on fouling are discussed in CHAPTER 5. A discussion on the impact of cleaning operation exclusion on fouling reduction is also included in CHAPTER 5 Section 5.6. 


\subsubsection{Model Development Phase}

The Model Development Phase was performed as the final phase of this research project. The objectives of this phase were as follows:

- To develop a statistical model based on the results of the $2^{4}$ factorial design of experiments.

- To evaluate the efficiency of the statistical model for the prediction of fouling by $2^{4}$ factorial design experiments performed in the Main Phase Experiments.

The results of the $2^{4}$ factorial design experiments used in the Main Phase Experiments were used to develop a statistical model for the determination of fouling of a submerged hollow fiber membrane given the operation/cleaning parameters for a fixed source water. The validity of the model was tested by comparing the level of fouling achieved during a 10 day long fouling experiment with the level of fouling predicted by the statistical model. Details on the development and evaluation of the statistical model are provided in CHAPTER 6. 


\section{CHAPTER 2 - Literature Review}

\subsection{Membrane Filtration Basics}

Stringent regulations requiring high quality of water for drinking purposes has resulted in the emergence of membrane filtration technologies as an effective treatment option for drinking water (Laine et al., 2000, Kabsch-Korbutowicz et al., 2006). Membrane filtration is a pressure driven process in which a pressure differential across a semi permeable membrane results in the separation of particulate and dissolved material from a solution such that the solution passes through the membrane while rejected materials stays at the opposing membrane surface. Rejection is selective and based mainly on the shape, size and charge of the matter needed to be filtered out of solution (Van der Bruggen et al., 2003). The filtered solution that passes through the membrane is called the permeate while the concentrated solution due to rejection of filtered material at the membrane surface is called the retentate (sometimes also called the concentrate) (Judd, 2011).

Membranes are characterized into four types based on the pore size of the membrane, the size of the material to be filtered as well as the pressure required to perform the filtration process (Van der Bruggen et al., 2003). Table 2.1 shows the characterization of membranes into microfiltration (MF), ultrafiltration (UF), nanofiltration (NF) and reverse osmosis (RO) based on the size of the membrane pores. 
Due to the different pore sizes, each membrane type is suited for the removal of specific particles such as small or large organic molecules, micro-organisms and even salts.

Table 2.1 - Characterization of membrane filters (adapted from AWWA, 2005 and Judd, 2011)

\begin{tabular}{|c|c|c|c|}
\hline $\begin{array}{c}\text { Membrane } \\
\text { type }\end{array}$ & Pore size & Rejection Capabilities & $\begin{array}{c}\text { Relative } \\
\text { Pressure } \\
\text { Requirement }\end{array}$ \\
\hline $\begin{array}{c}\text { Microfiltration } \\
\text { (MF) }\end{array}$ & $0.1-10 \mu \mathrm{m}$ & $\begin{array}{c}\text { Sand, cysts, bacteria and large (high } \\
\text { molecular weight) colloids }\end{array}$ & Lowest \\
\hline $\begin{array}{c}\text { Ultrafiltration } \\
\text { (UF) }\end{array}$ & $\begin{array}{c}1 \mathrm{~nm}-100 \\
\mathrm{~nm}\end{array}$ & $\begin{array}{c}\text { MF capabilities + viruses, small (low } \\
\text { molecular weight) colloids and } \\
\text { macromolecular dissolved organics }\end{array}$ & Low \\
\hline $\begin{array}{c}\text { Nanofiltration } \\
\text { (NF) }\end{array}$ & $\begin{array}{c}0.5 \mathrm{~nm}-1 \\
\mathrm{~nm}\end{array}$ & $\begin{array}{c}\text { UF capabilities + micro molecular } \\
\text { dissolved organics and some salts }\end{array}$ & High \\
\hline $\begin{array}{c}\text { Reverse } \\
\text { Osmosis (RO) }\end{array}$ & $<0.5 \mathrm{~nm}$ & $\begin{array}{c}\text { NF capabilities + free atoms, salts and } \\
\text { micro molecular dissolved organics }\end{array}$ & Highest \\
\hline
\end{tabular}

Microfiltration (MF) membranes have the largest pore size $(0.1-10 \mu \mathrm{m})$ of the four membrane types and thus have the highest permeability. As a result low pressures are sufficient enough to filter macro molecules such as suspended solids, protozoa and even bacteria from solution. The primary mechanism of filtration in MF processes is sieving through macropores in which particles larger than the pore size of the membrane are rejected at the membrane surface while smaller particles pass through (Van der Bruggen et al., 2003).

Ultrafiltration (UF) membranes have smaller pore sizes $(1 \mathrm{~nm}-100 \mathrm{~nm})$ than MF membranes and are capable of removing a larger range of macromolecules than MF. UF processes have proven to be useful for the removal of suspended solids from solution along with protozoa, bacteria and even viruses (Konieczny, 1998; Jacangelo et al., 2005; 
Arnal et al., 2009). Similar to the case of MF processes, UF filtration takes place via sieving through mesopores and requires low pressures for filtration (Van der Bruggen et al., 2003; Gao et al., 2011).

Due to the even smaller pore sizes $(0.5 \mathrm{~nm}-1 \mathrm{~nm})$ of nanofiltration (NF) membranes in comparison with UF, NF has the filtration capability of rejecting an even larger range of particle sizes than UF and have shown to be extremely efficient in removing micro pollutants like smaller sized organics (Costa and de Pinho, 2006) as well as pharmaceutically active compounds and pesticides (Van der Bruggen et al., 2008). The smaller pore sizes of NF membranes explain the low permeability of NF membranes compared to MF and UF membranes thus creating the need for higher pressures for the purpose of filtration. NF works by means of sieving through micropores as well as solution diffusion and particle rejection at the membrane surface via ionic interactions due to the presence of a surface charge on NF membranes (Judd 2011). As a result of the surface charge, NF can be used for the removal of ionic particles smaller than the pore size from solution (Van der Bruggen et al., 2003).

Compared to MF, UF and NF membranes, reverse osmosis (RO) membranes do not have predefined pores and thus sieving does not take place with RO membranes. Instead, these dense membranes filter dissolved particles out of solution via a solution diffusion process which depends on the solubility and diffusion rates of the particles and water through the membrane (Judd, 2011). Extremely high pressures are required for the purpose of RO and thus RO processes are extremely costly due to high energy consumptions (Van der Bruggen et al., 2003). RO processes produce extremely high quality of water by removing turbidity, microbial pathogens and all minerals from water 
(SDWF, 2009). Due to the high costs of producing high quality of water, RO filtration is mainly used in desalination plants for the production of drinking water from salt water.

While all four filtration processes are useful for separation of various constituents from source water, the rejection of these constituents ultimately results in the unavoidable phenomenon of membrane fouling which limits the practical use of these technologies Judd 2011). Membrane fouling (discussed further in Section 2.3) caused by the accumulation of constituents in source water on the membrane surface results in the reduction of permeability of the membrane and lower production of water. As a consequence, higher costs of treatment are incurred. Furthermore, production of higher qualities of water in turn results in higher costs of treatment. Thus in order to save costs, it is pertinent that appropriate processes are chosen based on the quality of source water to be treated as well as the specific regulatory requirements for the production of the treated water.

\subsection{Membrane Filtration in Water Treatment}

Valued at US \$1.4 billion in 2011 (BBC Research, 2011), membrane technology for the treatment of municipal drinking water has undergone significant advancements over the past two decades. While MF, UF, NF and RO, all produce high qualities of treated water, the low pressure MF and UF membranes are commonly used for surface water treatment due to the improvements of membrane materials, lower energy requirements and corresponding lower costs of treatment (Tian et al., 2010; Gao et al., 2011). MF membranes are very capable of removing turbidity causing particles, colloids 
as well as pathogenic bacteria and protozoa from surface waters. However, UF processes provide the added advantage of completely removing bacteria as well as most viruses from water (Van der Bruggen et al., 2003; Tian et al., 2010). The continuous progression of membrane fabrication and operating techniques has resulted in the rapid advancement and growing popularity of UF filtration of natural surface waters (Li et al., 2009). Due to this increasing popularity of UF treatment for the production of drinking water, UF technology in drinking water treatment will be the main focus of discussion for the remainder of this literature review.

\subsubsection{Operational Concepts}

An understanding of a number of operational concepts is necessary when dealing with low pressure membrane filtration in drinking water treatment. Firstly, the filtration flux $(J)$ is a measure of the rate of permeate flow through a membrane and is defined as the amount of permeate passing through unit area of a membrane per unit time $\left(\mathrm{m}^{3} / \mathrm{m}^{2} / \mathrm{s}\right.$ or $\mathrm{L} / \mathrm{m}^{2} / \mathrm{h}$ ) as shown in Equation 2.1. Membranes are often operated below a 'critical flux' as irreversible fouling is said to occur above this flux (Judd, 2011).

$$
J=\frac{Q_{\text {permeate }}}{A_{\text {membrane }}}
$$

Where,

$\mathbf{J}$

Qpermeate

Amembrane $\rightarrow \quad$ Filtration flux $\left(\mathrm{m}^{3} / \mathrm{m}^{2} / \mathrm{s}\right.$ or $\left.\mathrm{L} / \mathrm{m}^{2} / \mathrm{h}\right)$

$\rightarrow \quad$ Permeate flow $\left(\mathrm{m}^{3} / \mathrm{s}\right.$ or $\left.\mathrm{L} / \mathrm{h}\right)$

$\rightarrow \quad$ Surface area of the membrane $\left(\mathrm{m}^{2}\right)$ 
The transmembrane pressure (TMP) refers to the pressure differential between the feed side and permeate side of a membrane and is usually the driving force behind low pressure membranes (USEPA, 2005). Permeate flow through membranes can be achieved by operating the membrane system at either a constant membrane flux or a constant membrane pressure. During constant flux operations, constant flow through the membrane is maintained while the transmembrane pressure increases. The increase in transmembrane pressure occurs due to the reduced permeability and increased resistance experienced by the membrane as particles filtered out of solution accumulate on the membrane surface and foul the membrane. The opposite phenomenon takes place during constant pressure operations where the flux decreases over time due to membrane fouling while constant pressure is maintained through the membrane (AWWA, 2005).

Low pressure operations also make use of two main flow modes namely the deadend flow mode and the cross-flow mode. These flow modes are based on the 'recovery' of the system which is calculated as the ratio of permeate to feed flow of the system (Judd, 2011). During the dead-end flow mode, flow rate of feed provided to a membrane reactor tank is the same as the flow rate of permeate produced during treatment. As a result $100 \%$ recovery is achieved during dead-end flow mode (AWWA, 2005). However, the lack of a retentate stream when utilizing dead-end flow modes results in the accumulation of solids in the reactor tank over time. Thus system purges are required every so often to reduce the concentration of foulants in the reactor tank.

During crossflow mode, feed flow allowed to flow parallel to a membrane surface is greater than that of the permeate flow. As a result a retentate stream with a concentrated solution continuously flows out the membrane reactor (Judd, 2011). 
Permeate recovery when using a continuous flow mode typically ranges from 80 to $95 \%$ (Van der Bruggen et al., 2003; AWWA, 2005). The concentrated retentate in the crossflow mode is either recycled or needs to be disposed of. In drinking water treatment the components in the retentate stream are not usually toxic and thus a possible disposal technique is the discharge into water bodies if no toxic compounds are present. If the retentate has high organic content, the retentate can be evaporated and the remaining content incinerated (Van der Bruggen et al., 2003). A continuous flow (feed and bleed) operation is commonly used during a crossflow mode to regulate the rate of continuous waste stream (retentate) flowing out of the membrane reactor (Choi et al., 2005; USEPA 2005).

\subsubsection{UF - Membrane Configurations}

UF filtration makes use of a number of configurations made up of different kinds of membrane modules for the treatment of drinking water including tubular, flat sheet and hollow fiber membrane modules. Tubular membrane configurations involve a number of ceramic or polymeric membrane tubes with large inner diameters (up to 2 inches or 5 cm.) contained in a stainless steel or fiberglass re-enforced plastic casing (AWWA, 2005; USEPA 2005). Filtration is achieved via 'inside-out' permeate flow such that feed is pumped through the membrane tubes thus forcing permeate through the membrane pores and into the chamber between the membranes and inner wall of the steel/plastic casing (AWWA, 2005). A high velocity of feed is required to minimize particle deposition on the membrane surface and prevent membrane fouling. In tubular membranes with higher 
inner diameters, the higher velocities could result in high cost of treatment (Wagner, 2001).

In flat sheet membranes, also known as plate and frame membranes, a membrane module is made up of a long polymeric flat membrane sheet attached to a plate such that a permeate collection chamber is created between the membrane and plate. A number of flat sheet membranes modules are then assembled side by side using feed-flow spacers into one large unit such that an adequate channel space is present between each module for crossflow of feed solution along the membrane surface (Hendricks, 2011). The unit is submerged in feed solution and filtration is performed by creating a vacuum in the collection chamber of each module thus causing permeate to flow through the membrane pores into the collection chamber. This flow of permeate from outside the membrane to the inside of the membrane module is known as an 'outside-in' permeate flow regime. Coarse bubble aeration can be provided between channels to ensure scouring of the membrane surface and prevent fouling. While this UF membrane configuration has good fouling control, the membranes cannot be backwashed and rely on chemical cleaning for fouling removal (Judd, 2002).

Hollow fiber membrane configurations make use of a large number $(10,000$ 20,000 per unit process on a commercial scale) of long narrow polymeric tubes called hollow fiber membranes bundled together and potted in epoxy or resin at both ends (AWWA, 2005; Hendricks, 2011). Permeate flow through hollow fiber membranes can be achieved by either applying pressure or vacuum within the module i.e. both 'outsidein' and 'inside-out' permeate flow regimes are possible (AWWA, 2005). The production of hollow fiber membranes is much less expensive compared to flat sheet membranes. 
While the hollow fiber membrane configuration is prone to fouling, the useful fouling reducing technique of backwashing and air scouring can be performed on hollow fiber membranes (Judd, 2002).

The use of these three membrane modules in water treatment is governed by costs of production as well as costs associated with reducing fouling of the membrane modules. While flat sheet membranes experience less fouling due to the low filtration fluxes used for treatment, these membranes require chemical cleaning (Judd, 2002) or shearing stresses using high crossflow velocities (Shon et al., 2006) to remove fouling, both of which are expensive. As previously mentioned, operational costs for tubular membranes are high due to the high energy required to provide crossflow velocities to ensure membrane permeability and fouling reduction. Furthermore, the efficiency of both treatment systems are compromised due to the poor membrane surface area to volume ratio and thus are not as popular in the treatment of water treatment (USEPA, 2005). Hollow fiber membranes on the other hand have the highest surface area to volume ratio of the three aforementioned membranes. Hollow fiber membranes are also cheap to produce and can be cleaned using physical methods such as air scouring and backwashing thus making them the most commonly used membrane configuration for the treatment of water (AWWA, 2005). While physical cleaning methods could be expensive, cost can be reduced by optimizing the frequency and duration of these cleaning techniques. The high efficiency of treatment along with the decreasing costs of energy for treatment of water has resulted in the recognition of hollow fiber UF technology in water treatment as the 'Key technology of the $21^{\text {st }}$ Century' (Tian et al., 2010). 


\subsubsection{Application of UF in Water Treatment}

UF treatment is capable of consistently producing high quality waters regardless of changes in source water quality (Green and Tylla, 1998; Arnal et al, 2009). However, the quality of source water does determine if the water can be treated using UF directly or if UF needs to be combined with other processes to optimize the removal of contaminants from the water as well help control membrane fouling (AWWA, 2005). Direct UF treatment has been found to be effective in removing macro molecules and turbidity causing particles as well as pathogenic microorganisms like bacteria, viruses and protozoa (Cryptosporidium and Giardia) without the need of any pre-treatment (Green and Tylla, 1998; Van der Bruggen et al., 2003). In such cases where the requirement is only the removal of turbidity and pathogenic microorganisms from a high quality source water, conventional drinking water treatment processes including coagulation, flocculation, sedimentation, filtration and even disinfection can all be replaced by a single UF treatment process (Van der Bruggen et al., 2003; Tian et al., 2010). Since fewer units are required for treatment, the use of UF treatment reduces the complexity of the treatment system and consequentially improves system operability (Daigger et al., 2006).

Along with the advantage of a reduced footprint due to the compactness of the treatment facility required, UF treatment holds numerous advantages over conventional treatment including the production of a higher quality of water produced as well as the reduced amount of chemicals required during treatment (Nakatsuka et al., 1996; Arnal et al, 2009). A comparison of the particle removal efficiency using a conventional treatment system (micro-straining followed by rapid sand filtration) and UF showed that while the conventional treatment was able to remove $90 \%$ of particles from the source water, UF 
was able to remove $99 \%$ of the particles from the water (Lipp et al., 1998). Also, as previously mentioned UF treatment is efficient in removing pathogenic micro-organisms from surface waters and thus provides a level of disinfection. As a result fewer chemicals are required for the disinfection of water after treatment resulting in lower treatment costs as well as the possibility of fewer disinfection by-products in the produced water. The use of UF to treat water directly also eliminates the need for chemical coagulants and flocculants used during conventional treatment and thus reduces costs.

While UF can effectively remove turbidity causing particles and microbial pathogens from surface water, the technology is not efficient in removing micro molecular organics such as low molecular weight humic substances, pesticides and chlorinated solvents from source waters (Anselme et al., 1994; Tomaszewska and Mozia, 2002). On the other hand, macro molecular organics that can be removed using UF such as high molecular weight humic substances and soluble microbial products (SMP) are known to foul membranes (Chen et al., 2007; Katsoufidou et al., 2008). Thus, for source waters with high organic content, treatment is usually performed by integrating UF processes with conventional treatment processes such as coagulation (Chen et al., 2007), powdered activated carbon (Tomaszewska and Mozia, 2002) and/or biofiltration (Mosqueda-Jiminez et al., 2008) such that pre-treatment of the source water is performed before UF to enhance the removal of organics as well as minimize organic fouling of the membranes (AWMA, 2005).

Another application of UF in drinking water is the application of UF along with biological processes in submerged membrane bioreactors (sMBR) for the effective removal of high ammonia and organic levels from highly polluted sources waters. This 
new emerging treatment using UF has been gaining popularity due to a number of advantages over conventional biological treatment including the effective removal of organic disinfection by-product (DBP) precursors along with the consequential reduction of DBPs like trihalomethanes (THMs) while requiring a simpler flow configuration and smaller footprint for the production of drinking water (Li and Chu, 2003; Tian et al., 2009). However, as with all membrane treatment systems, the application of submerged MBRs for the treatment of drinking water is limited by membrane fouling.

\subsection{Membrane Fouling}

The use of UF in drinking water continues to show great promise for the removal of contaminants such as turbidity causing particles, organic matter as well as pathogenic microorganisms in source waters (Hagen, 1998; Hillal et al., 2005; Gao et al., 2011). However, during the filtration process, an unavoidable phenomenon known as membrane fouling takes place. Contaminants rejected by the membrane during filtration tend to accumulate on the membrane surface and within pores resulting in a reduction of the membrane permeability and an increase in the resistance to permeate flow (Nakatsuka $e t$ al., 1996; Bottino et al., 2009; Gao et al., 2011). Consequently, the production of permeate suffers and higher energy is required to compensate for losses in permeate production due to fouling (Nakatsuka et al., 1996; Yamamura et al., 2007). Furthermore, permeate production losses are also experienced due to the water requirements of physical fouling control techniques like backwashing ( $\mathrm{Li}$ and $\mathrm{Chu}, 2003$ ). 
Two main mechanisms of fouling exist in the UF treatment of drinking water namely pore blocking and cake formation (Hilal et al., 2005; Gao et al., 2011). Pore blocking is the initial fouling mechanism during which particles smaller than the pore size of the membrane adhere to the walls of the membrane pores via adsorption. Due to the membrane particle interactions causing adsorption, this mechanism of fouling is usually physically irreversible i.e. the fouling cannot be removed using physical cleaning method of backwashing (Hilal et al., 2005; Guo et al., 2009). Irreversible fouling (Figure 2.1b) explains the gradual increase in TMP over time even when physical cleaning methods are employed for fouling reduction (Gao et al., 2011). Chemical cleaning is thus required to recover permeate flux caused by irreversible fouling (Hilal et al., 2005).

Cake formation usually follows pore blocking and involves the deposition of larger particles on the surface of the membrane due to concentration polarization.(Hilal et $a l ., 2005)$. In concentration polarization, larger particles drawn to the membrane surface during filtration tend to remain near the surface after rejection. Over time, the concentration of these particles increases in the immediate vicinity of the membrane surface resulting in the precipitation and accumulation of particles on the membrane surface (Judd, 2011). Since the formation of cake layer is mostly governed by filtration and not charge interactions, this mechanism of fouling is reversible and can be removed using physical cleaning techniques as illustrated in Figure 2.1a (Aoustin et al., 2001; Hilal et al., 2005; Guo et al., 2009). 
Figure 2.1 - Schematic of a) Reversible and b) Irreversible fouling

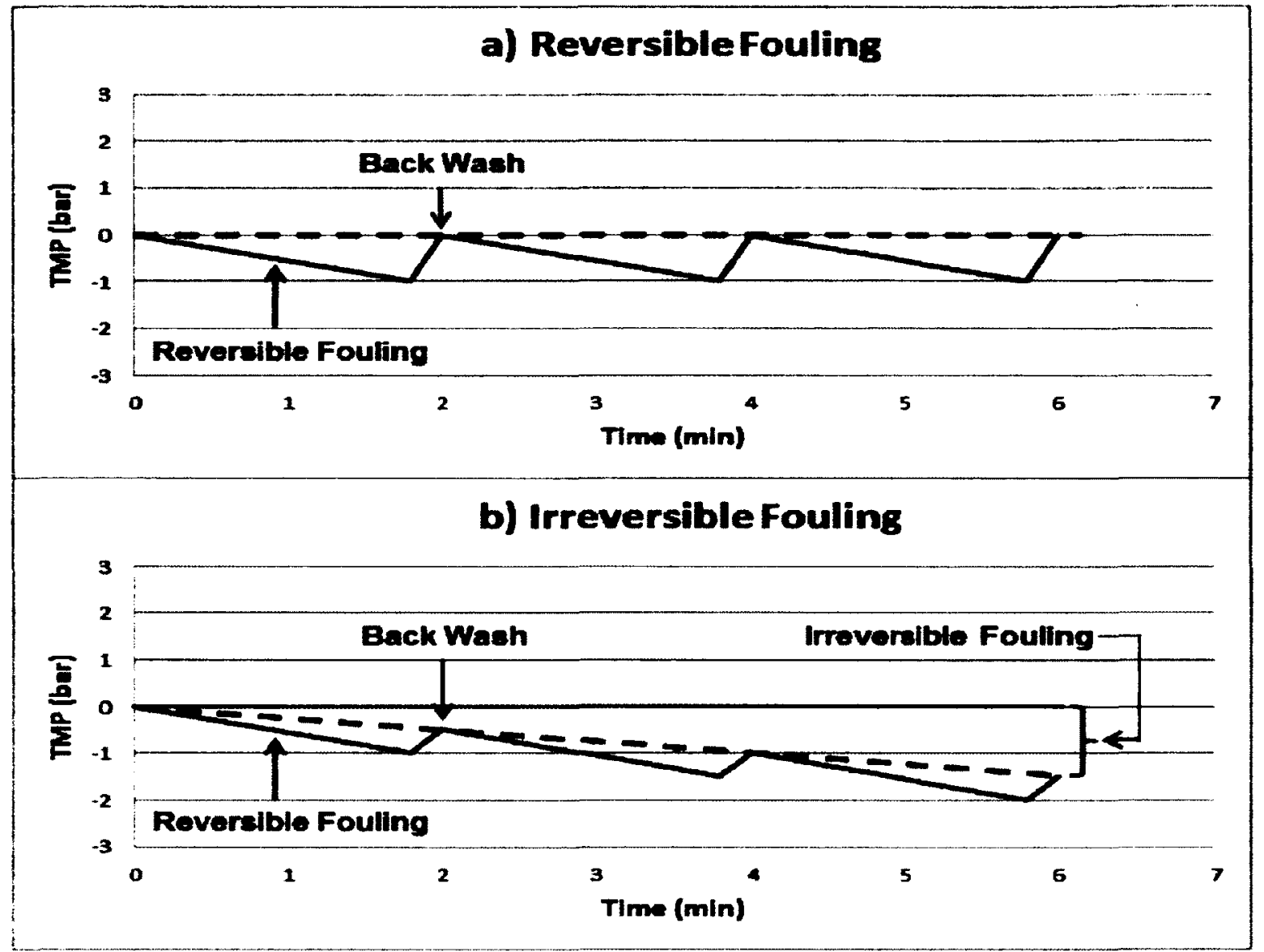

UF membrane fouling depends on a number of factors including the characteristics of source water constituents, the characteristics of the membrane itself as well as interactions between the membrane surface and source water constituents (Li et al., 2010). Filtration flux (Amy, 2008; Yigit et al., 2009) and flow modes (Choi et al., 2005; Mendret et al., 2009) have also been found to effect fouling behavior. The various factors effecting fouling in the treatment of drinking water are discussed in Sections 2.3.1 $-2.3 .3$ 


\subsubsection{Source Water Characteristics}

Common constituents of natural source waters include turbidity causing inorganic particles, natural organic matter (NOM) and microorganisms which provide major contributions to particle fouling, organic fouling and biofouling respectively (Hilal et al., 2005; Guo et al., 2009). Studies have also shown that ions present in source water such as $\mathrm{Ca}^{2+}$ and $\mathrm{Na}^{+}$contribute to fouling by creating a bridging effect while attaching foulants to the membrane surface (Aoustin et al., 2001; Katsoufidou et al., 2008; Li et al., 2009).

\subsubsection{NOM Fouling}

Natural organic matter (NOM) in surface waters is a highly complex matrix of organics made up of humic substances, proteins, carbohydrates and fatty acids and is considered as a primary contributor to fouling in drinking water treatment (Yamamura $e t$ al., 2007; Guo et al., 2009). As such, the prediction of fouling by NOM is extremely difficult (Zularism et al., 2011). For the purpose of understanding fouling, NOM is fractionated into a hydrophobic fraction consisting of humic substances and a hydrophilic fraction which includes carbohydrates, proteins and fatty acids (Yamamura et al., 2007; Zularism et al., 2011).

Humic substances are a result of biodegradation processes in nature and are highly complicated heterogeneous structured organic molecules, the characterisation of which is extremely difficult (Domany et al., 2002). Humic substances which make up a major fraction of NOM are further classified into humic acids (HA), fulvic acids (FA) and humin based on molecular weight and solubility in water (Aoustin et al., 2001, 
Domany et al., 2002, Zularism et al., 2006). While FA are generally soluble in water, HA precipitates out of water at a low $\mathrm{pH}(<2)$ and humin are non-soluble. Of the three humic substances, HA are of great concern during fouling due to the hydrophobic nature of HA as well as the large size (2000 to 5000 Daltons) associated with the hydrophobicity compared to FA (Aoustin et al., 2001, Li et al., 2010).

Most interactions between membranes and foulants are hydrophobic in nature (Matosic et al., 2005). As such, the hydrophobic nature of HA promotes the adsorption of HA onto the typically hydrophobic membrane surface and within membrane pores resulting in significant fouling (Aoustin et al., 2001). Li et al. (2010) investigating the effect of HA on membrane fouling found that backwashing was unable to recover the TMP of the membrane showing that HA was responsible for irreversible fouling due to pore blocking by adsorption and gel layer formation. Similar pore blocking by HA causing irreversible fouling was noticed in studies by Aoustin et al. (2001) and Yamamura et al. (2007). In addition, HA fouling via adsorption was thought to be responsible for high fouling caused by hydrophilic NOM. Yamamura et al. (2007) postulated that the high fouling by hydrophilic NOM (carbohydrates) was due to the blocking of HA constricted pores by the NOM. This pore blocking was thought to be irreversible. HA can also cause cake layer fouling by aggregation and accumulation of material at the membrane surface. This effect is noted under high ionic strengths and is usually reversible (Aoustin et al., 2001).

The hydrophilic fraction of NOM consisting of soluble microbial products (SMP) such as carbohydrates, proteins and fatty acids are created by microbial activity (Metzger et al., 2007; Amy, 2008; Judd, 2011). While the hydrophobic fraction of NOM was 
thought to be the dominant foulant of drinking water treatment, studies have shown that the hydrophilic fraction results in more fouling than hydrophobic fraction of NOM (Zularism et al., 2006; Yamamura et al., 2007; Zularism et al., 2011). SMP fouling occurs by the formation of a gel layer on the membrane or via hydrophilic interactions between the hydrophilic surface of membrane fouled by hydrophobic NOM (Metzger $e t$ al., 2007; Yamamura et al., 2007).

\subsubsection{Inorganic Particulate Fouling}

Inorganic turbidity causing particulates in surface waters are comprised mainly of clays that are larger than the pore sizes of the membranes. As a result, these inorganic particles are rejected at the membrane surface due to size exclusion. Fouling of membranes by inorganic particles is thus due to accumulation of the particles on the membrane surface resulting in the formation of a cake layer. While increases in turbidity result in a faster rate of fouling, fouling contribution by inorganic particles is less than that produced by organic particles and is reversible (Li et al., 2010; Zularism et al., 2011). Furthermore, Li et al. (2010) found that the presence of inorganics in solution helped lower the HA fouling by reducing the compactness of cake layer formation.

\subsubsection{Membrane characteristics}

With respect to the membrane itself, fouling is usually dependent on the pore size of the membrane as well as the hydrophobicity of the membrane surface. Membranes with smaller pore sizes are known to be more susceptible to irreversible fouling than 
membranes with larger pore sizes (Amy, 2008). UF membranes for the treatment of water are usually made of hydrophobic material like polyvinylidene fluoride (PVDF) (Van der Bruggen et al., 2003; Shobana et al., 2010). However, such membranes are prone to fouling due to interactions between the hydrophobic membrane surface and hydrophobic

foulants like HA and other humic substances (Matosic et al., 2008). Furthermore, hydrophilic membranes are known to exhibit reduced fouling effects and increased fouling reversibility (Katsoufidou et al., 2008). Thus, fouling effects of hydrophobic membranes are usually reduced by lowering the hydrophobicity of the membrane surface using physical and chemical means of surface modifications such as surface coating with hydrophilic polymers and grafting using hydrophilic monomers respectively (Van der Bruggen et al., 2003; Hilal et al., 2005; Shobana et al., 2010).

\subsubsection{Filtration flux and flow modes}

As logic would dictate, increases in the filtration flux results in a higher rate of fouling via foulant accumulation on the membrane surface. This increase in filtration flux causing fouling is the basis of flux step models used to determine the onset of irreversible fouling known as the critical flux of membranes (Le Cleche et al., 2003). The duration of filtration flux is also known to increase fouling as reported by Yigit et al. (2009) where the fouling effects of long filtration fluxes were found to outweigh the fouling reducing effects by the cleaning operation of backwashing.

With regards to the effect of flow modes on fouling behavior, the use of a deadend flow mode of operation results in the continuous accumulation of particles on the 
membrane surface. As such, continuous fouling is accompanied by an increasing thickness of the fouling layer which is responsible for decreases in productivity of the membrane (Hong et al., 2005; Mendret et al., 2009). While the dead-end flow mode is the most economical flow mode used in drinking water treatment (Mendret et al., 2009), the continuous accumulation of foulants on the membrane surface requires frequent backwashing which may not be entirely effective at reducing fouling due to the enhanced concentration polarization of foulants at the surface of membranes after backwashing (Choi et al., 2005; Mendret et al., 2009). Alternatively, the use of a crossflow mode with continuous flow operation has shown positive effects on fouling reduction mainly due to the minimization of concentration polarization at the membrane surface (Choi et al., 2005).

\subsection{Fouling Attenuation}

While physical and chemical modifications have been effective in reducing the hydrophobicity of membrane surfaces thus making the membrane less prone to fouling, membrane fouling still occurs (Hilal et al., 2005). Thus additional fouling attenuation methods have been developed over the years to further reduce fouling and increase the efficiency of treatment using UF membranes. These methods of fouling reduction include pre-treatment of the source water prior to UF, chemical cleaning of the membranes specifically for the removal of irreversible fouling and physical cleaning techniques such as backwashing, relaxation and air scouring which mainly target reversible fouling (Hilal et al.; 2005, Gao et al., 2011). 


\subsubsection{Feed Pre-treatment}

Pre-treatment of the source water prior to UF is commonly performed to remove particulates and organic matter that might contribute to fouling of the UF membrane during drinking water treatment (Hilal et al., 2005; Kabsch-Korbutowicz et al., 2006). One of the more commonly used methods of pre-treatment used is inline coagulation ( $\mathrm{Li}$ et al., 2010). While coagulation has been useful in removing organics and particles during pre-treatment, the process may not always be appropriate as a pre-treatment technique. Studies have reported that coagulation is not efficient in the removal of hydrophilic NOM that may cause irreversible fouling in UF (Kabsch-Korbutowicz et al., 2006; Gao et al., 2011). Furthermore, a large amount of waste sludge with high concentrations of aluminum and iron are produced due to periodic backwashing of UF membranes. Treatment of the waste sludge before being disposed is required and thus high costs are incurred (Li et al., 2010).

\subsubsection{Chemical Cleaning}

Chemical treatment is essential for all membrane treatment processes specifically for the removal of irreversible fouling and the consequential recovery of membrane permeability (Hilal et al., 2005, Porcelli and Judd, 2010). Chemical cleaning typically makes use of chemicals like sodium hypochlorite and citric acid for the removal of organic and inorganic fouling respectively. Cleaning is performed by either introducing these chemical in a chemically enhanced backwash (CEB) or by using a clean in place (CIP) treatment step (Judd, 2011). While chemical cleaning is necessary for fouling 
attenuation, frequent usage of chemical cleans can cause irreversible damage to the membranes and shorten their life span (Van der Bruggen et al., 2003; D'Souza and Mawson, 2005; Wang et al., 2010). Cleaning using sodium hypochlorite as a cleaning agent has been known to increase the effective pore size of the membranes (Wolff and Zydney, 2004; Lu et al., 2011). Sodium hypochlorite has also been responsible for removal of surface modifications and thus changes to the overall membrane properties (Puspitasari et al., 2010; Wang et al., 2010). Furthermore, hypochlorite cleaning weakens and makes the membranes more flexible (Wang et al., 2010). Of course, the negative effect of sodium hypochlorite on membranes is dependent on the concentration and amount of exposure used during cleaning as well as the membrane material (Hilal et al, 2005).

Since physical cleaning usually precedes chemical cleaning, the proper optimization of physical cleaning operations might prove to be beneficial for the purpose of reducing the frequency of chemical cleaning and maintaining the wellbeing of the membrane. Furthermore the lower use of chemicals is ideal from a cost benefit point of view (Gao et al., 2011).

\subsubsection{Physical Cleaning}

A number of physical cleaning operations have been developed over the years to reduce fouling in membranes, the two most common of which are air scouring and backwashing. Relaxation is a passive cleaning step and while its use is rarely mentioned during the treatment of drinking water, it is a staple process in wastewater treatment 
using membrane bioreactors (Wu et al., 2008). Sections 2.4.3.1 to 2.4.3.3 discuss the useful fouling removal mechanisms brought about by the three physical cleaning methods.

\subsubsection{Backwashing}

Backwashing is a common practice employed during membrane filtration due to its efficiency in restoring membrane permeability by removing most reversible pore blocking as well as dislodging the cake layer off the membrane surface (Katsoufidou et al., 2008; Yigit et al., 2009). Backwashing involves the periodic backflow of permeate through the membrane over a short time frame such that the reversible foulants are flushed out of the membrane pores, off the membrane surface and back into the feed solution. While backwashing helps to restore membrane permeability, it can also be the cause of additional fouling by removing the cake layer and promoting pore blocking (Metzger et al., 2007). Small particles are known to diffuse towards the membrane surface at a faster rate than larger particles (Aoustin et al., 2001). The constant removal of the cake layer by backwashing thus allows for the continuous adsorption of smaller particles on the membrane surface and in membrane pores at the start of filtration periods causing pore blocking and irreversible fouling (Metzger et al., 2007).

Backwash efficiency depends on a number of factors including backwash duration, backwash interval and backwash flux (Wu et al., 2008). In addition air scouring with backwashing (air assisted backwashing) has been shown to improve fouling mitigation (Serra et al., 1999; Remize et al., 2010). Table 2.2 shows a summary of 
findings from various studies investigating the effect of backwashing on fouling reduction in water treatment as well as wastewater treatment. While the quality of water treated during wastewater treatment is quite different from source waters for drinking water treatment, the general findings from these studies are insightful and may apply in either scenario.

The proper frequency and duration of backwashing are extremely important to prevent additional fouling of the membrane by instantaneous fluxes used after backwashing to compensate for losses in permeate productivity (Braak et al., 2011). However, on viewing Table 2.2 it can be seen that no clear cut standard for the duration and interval between backwashes exist in the treatment of water or wastewater. Furthermore, different feed qualities and backwash parameters resulted in contradicting effects of backwash duration, interval and flux on fouling. A commonality in most cases was the efficiency of backwashing in removing fouling from membranes especially when assisted by air scouring. 
Table 2.2 - Summary of findings from studies investigating the effect of backwashing (BW) and air assisted backwashing on fouling.

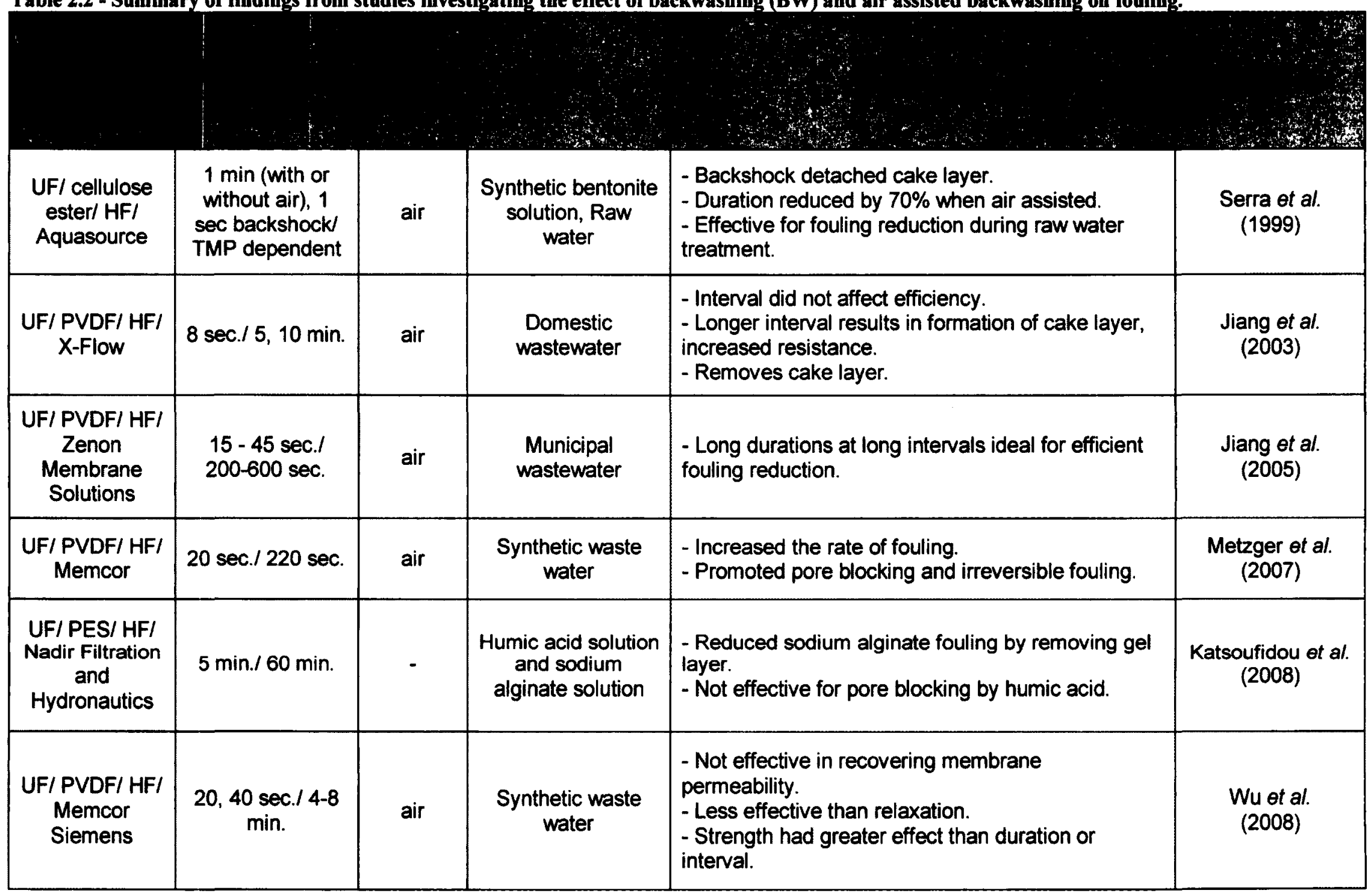




\begin{tabular}{|c|c|c|c|c|c|}
\hline $\begin{array}{l}\text { UF/ PVC/ HFI } \\
\text { Litree } \\
\text { Company }\end{array}$ & $\begin{array}{l}3 \mathrm{~min} \text {. and } 2.75 \\
\text { min. (when } \\
\text { assisted by } \\
\text { flushing)/ end of } \\
\text { fouling }\end{array}$ & $\begin{array}{l}\text { hydraulic } \\
\text { flushing }\end{array}$ & $\begin{array}{l}\text { Hydrophobic DOM } \\
\text { solutions (Fulvic } \\
\text { acid, Tannin and } \\
\text { Aniline sols.) }\end{array}$ & $\begin{array}{l}\text { - Efficient in removing cake formation and pore } \\
\text { blocking by fulvic acid and analine when assisted by } \\
\text { flushing. } \\
\text { - Not effective in removing pore blocking by tannin. }\end{array}$ & $\begin{array}{c}\text { Guo et al. } \\
(2009)\end{array}$ \\
\hline $\begin{array}{l}\text { MF/ ceramicl } \\
\text { Tubular/ Orelis } \\
\text { Co. }\end{array}$ & $\begin{array}{l}2,4,8 \min . / 15 \\
\min .\end{array}$ & - & $\begin{array}{l}\text { Polymethyl } \\
\text { methacrylatede } \\
\text { (5um) in de-ionized } \\
\text { water }\end{array}$ & $\begin{array}{l}\text { - Eliminates particle fouling, increases productivity. } \\
\text { - Durations }>2 \text { min. may decrease productivity. } \\
\text { - Effective for the removal of cake layer. } \\
\text { - Increase in strength and duration improves fouling } \\
\text { reduction. }\end{array}$ & $\begin{array}{c}\text { Hwang et al. } \\
(2009)\end{array}$ \\
\hline $\begin{array}{l}\text { UF/ PVDF/ HF/ } \\
\text { Zenon } \\
\text { Membrane } \\
\text { Solutions }\end{array}$ & $\begin{array}{c}5-20 \mathrm{sec} . / 4.75 \\
-59.75 \mathrm{~min} .\end{array}$ & air & $\begin{array}{l}\text { Screened raw } \\
\text { domestic waste } \\
\text { water }\end{array}$ & $\begin{array}{l}\text { - Effective in reducing fouling. } \\
\text { - Interval has a greater impact than duration. } \\
\text { - Shorter intervals resulted in less fouling. }\end{array}$ & $\begin{array}{l}\text { Yigit et al. } \\
\text { (2009) }\end{array}$ \\
\hline $\begin{array}{c}\text { UF/ polymeric/ } \\
\text { HFI } \\
\text { Aquasource }\end{array}$ & $\begin{array}{l}40 \mathrm{sec} .(20 \mathrm{sec} \text {. } \\
\text { air assisted }+20 \\
\text { sec. no air }) / 30 \text {. } \\
20 \mathrm{~min} .\end{array}$ & air & $\begin{array}{l}\text { Clay suspension; } \\
\text { Natural surface } \\
\text { water }\end{array}$ & $\begin{array}{l}\text { - Effective in reducing fouling. } \\
\text { - Particles remaining after backwashing were } \\
\text { pushed away from membrane surface by air. }\end{array}$ & $\begin{array}{l}\text { Remize et al. } \\
\qquad(2010)\end{array}$ \\
\hline $\begin{array}{l}\text { UF/ PVDF/ HF/ } \\
\text { Siemens } \\
\text { Water } \\
\text { Technology }\end{array}$ & $\begin{array}{c}30 \mathrm{sec} . / 59.50 \\
\mathrm{~min} .\end{array}$ & air & $\begin{array}{c}\text { Bentonite + } \\
\text { alginate solution }\end{array}$ & $\begin{array}{l}\text { - Cannot remove foulant layer by itself. } \\
\text { - Longer duration required if unassisted by air. } \\
\text { - Slightly better than relaxation in reducing fouling. } \\
\text { - Only loosens foulants, air required to remove } \\
\text { foulants off surface. }\end{array}$ & $\begin{array}{l}\text { Ye et al. } \\
\text { (2011) }\end{array}$ \\
\hline
\end{tabular}




\subsubsection{Relaxation}

While commonly used in membrane bioreactors for the treatment of wastewater (Wu et al., 2008), the use of relaxation in drinking water is less documented. Relaxation involves the periodic pausing of filtration for a set time period to allow for back transport of foulants off the membrane surface and into the feed due to the presence of a concentration gradient (Hong et al., 2002; Braak et al., 2011). As in the case of backwashing, the efficiency of relaxation depends on the duration and interval of relaxation (Wu et al., 2008). Short and less frequent relaxations have been found to help reduce fouling while long and frequent relaxations have proved to be ineffective for fouling reduction due to the required instantaneous fluxes that usually negate the fouling reducing effect of relaxation (Wu et al., 2008; Braak et al., 2011). Relaxation is also greatly impacted by air scouring (Hong et al., 2002; Ye et al., 2011). Table 2.3 summarizes key findings from recent studies investigating the effect of relaxation on fouling control albeit in a mostly wastewater treatment context.

Comparing the findings in Table 2.3, it can be seen that air assisted relaxation is capable of reducing reversible fouling by controlling cake layer formation. However, relaxation is not the primary provider of the fouling control mechanism and only helps loosen the cake layer from the membrane surface (Ye et al., 2011). It should also be noted that the use of air assisted relaxation resulted in similar and even better fouling reduction than the use of air assisted backwashing (Shon et al, 2006; Metzger et al, 2007). As in the case of backwashing, relaxation whether air assisted or not, cannot remove irreversible fouling (Hong et al., 2002). 
Table 2.3 - Summary of findings from studies investigating the effect of relaxation on fouling

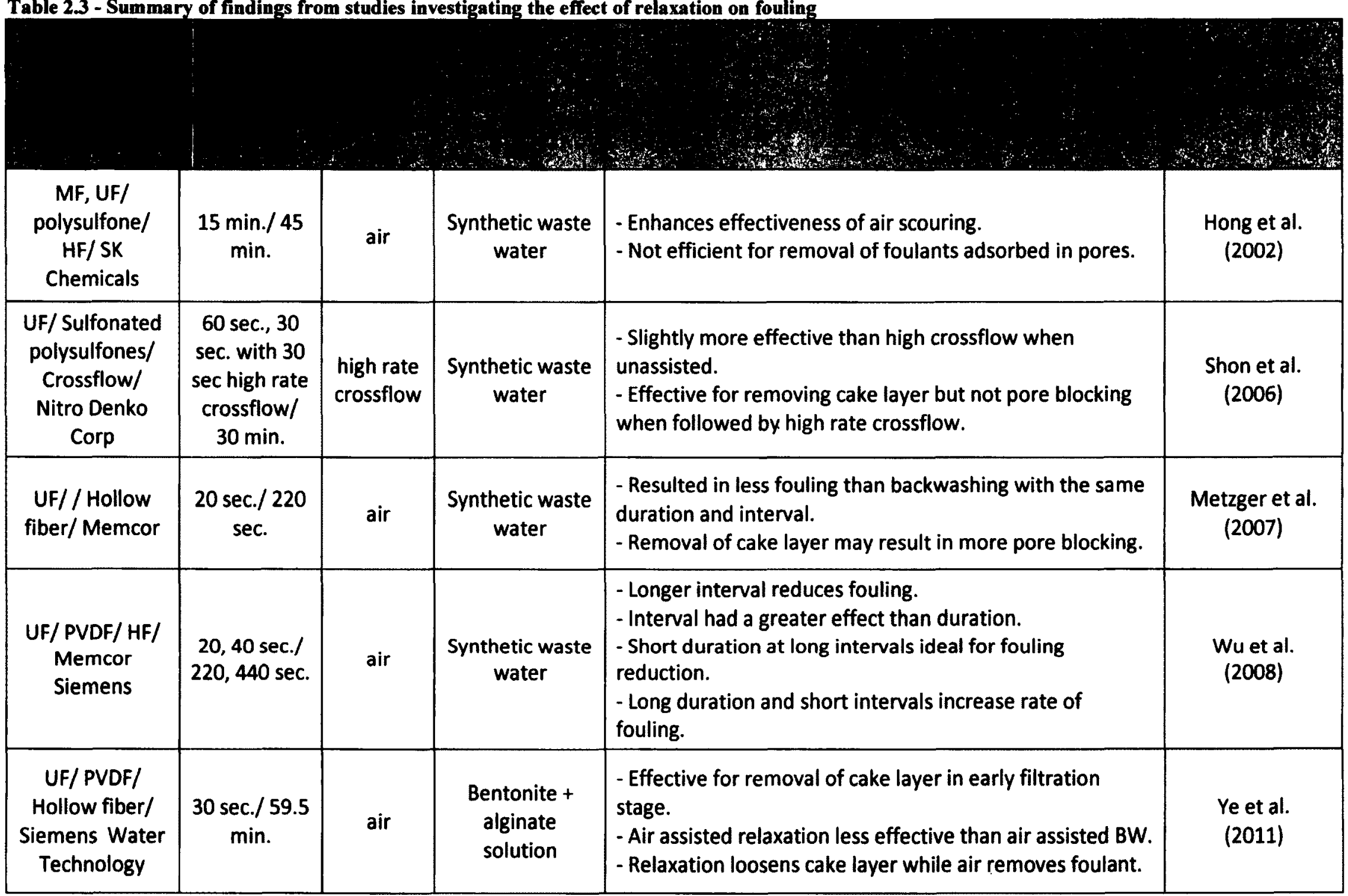




\subsubsection{Air Scouring}

Of the three physical cleaning regimes discussed in this review, air scouring is the most effective method of controlling cake layer formation on the membrane surface and plays a dominant role in the reduction of fouling by air assisted backwashing and air assisted relaxation (Ye et al., 2011). In air assisted relaxation and backwashing, air scouring is responsible for the movement of foulants away from the membrane surface once they are loosened by relaxation and backwashing and thus reduces fouling by controlling the cake layer formation (Cabassud et al., 2001).

Air scouring makes use of a number of mechanisms to reduce fouling on the membrane surface. The main mechanism of fouling control is the surface shearing by air bubbles as they rise along the membrane surface resulting in the removal of cake layer off the surface (Hong et al., 2002; Cui et al., 2003; Gao et al., 2011). Air flow rate plays a key role during surface shearing as increasing flow rates result in higher fouling reduction (Hong et al., 2002; Tian et al., 2010). Also, continuous air scouring was found to be more efficient at controlling fouling than intermittent air scouring (Tian et al., 2010). However the use of intermittent fouling is more cost effective (Cui et al., 2003).

Along with the shear from the bubbles, movement of bubbles along the surface results in a two phase flow as water is dispersed by the bubbles promoting back transport of foulants from the membrane surface (Cui et al., 2003; Braak et al., 2011). Back transport is also influenced by the movement of membrane fibers due to the turbulent flow produced (Braak et al., 2011). The extent of the secondary flow produced and its effect on fouling reduction is highly dependent on the size of bubbles used for air 
scouring (Cui et al., 2003). While Cui et al. (2003) reported that coarse (1.5 - $15 \mathrm{~mm})$ ellipsoidal bubbles were ideal for fouling attenuation using air scouring, smaller bubbles were found to be effective for the purpose of fouling mitigation (Yeo et al., 2006; Tian et al., 2010). 


\section{CHAPTER 3 - Methodology}

A bench scale hollow fiber membrane filtration set up was used for this research. A schematic of the experimental setup as well as information on the various instruments used in the setup is provided in Section 3.1.1 of this chapter. The constituents of the simulated test water used for all experiments are included in Section 3.1.2. The various study phases carried out during the research study presented in a flow chart in Section 3.2 while the various data analysis procedures including the analysis of variance (ANOVA) model, interaction plots, general linear modelling and step wise linear regression used in the study are presented in Section 3.5.

\subsection{Materials and Methods}

\subsubsection{Apparatus Setup}

The bench scaled hollow fiber membrane filtration setup used for all experiments made use of a ZeeWeed ZW-1 (GE/Zenon) hollow fiber membrane module which is ideal for bench scaled fouling/cleaning studies. These polyvinylidene fluoride (PVDF) membranes have a membrane chemistry suited for the filtration of source water for drinking water treatment. The membranes are reported to have a nominal pore size of $0.04 \mu \mathrm{m}$ and an effective membrane surface area of $0.047 \mathrm{~m}^{2}$ per module. The membrane module also featured a built in air scouring system in which air in the form of coarse 
bubbles rise up from the base of the membrane module between membrane fibers to help prevent contaminants from settling on the membrane surface

Additional membrane specifications can be seen in APPENDIX A. A schematic of the apparatus used in this study can be seen in Figure 3.1. The set up featured three flow lines; an Air flow line, a Feed flow line and a Permeate flow line. Initially a single setup (i.e. one membrane) was used for preliminary experiments. Due to the successful working of the apparatus in the preliminary tests, a second setup was installed to maximise the number of experiments under the study. The second setup was identical to the initial setup except for the model of the flow meter used in the Air flow line. At the time of duplication of the experimental setup, the electronic flow meter by Differential Pressure Technologies used in the initial setup was discontinued. Thus an electronic flow meter by Aalborg with similar flow measuring capabilities was used in its place.

Figure 3.1 - Schematic of the bench scale membrane filtration setup

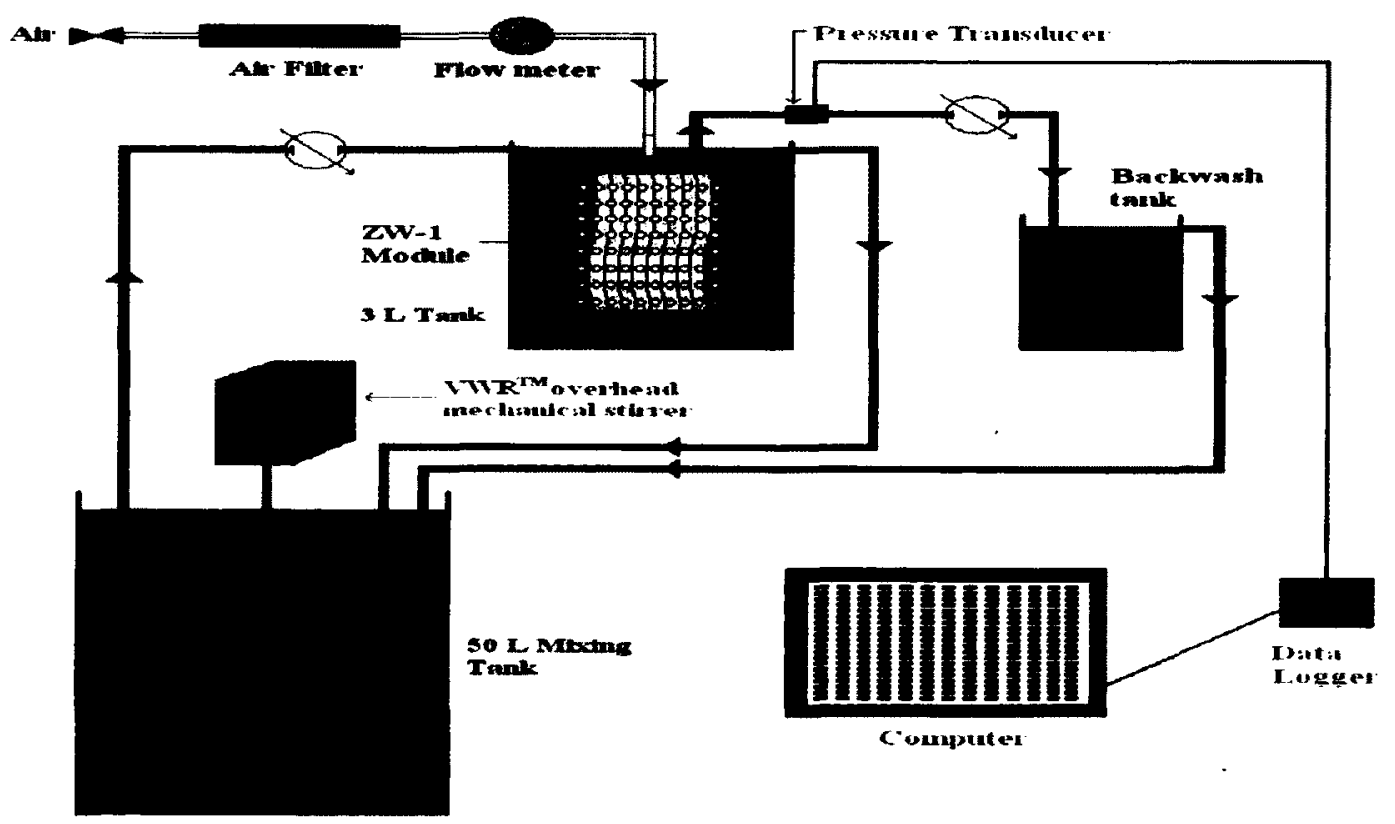


Materials and instruments incorporated into the Air flow line included a flow modulator, a Whatman HEPA-VENT ${ }^{\mathrm{TN}}$, an electronic flow meter with a digital LCD readout (Differential Pressure Technologies or Aalborg GFM), Cole-Parmer tubing (Cflex and FEP rigid tubing), Cole-Parmer polypropylene straight and reducing unions, a plastic valve, a Boyu flexible air curtain (Shenzhen Vakind Technology Co., Ltd) and the built in air scouring system of the ZW-1 module. A detailed schematic of the air flow line can be seen in APPENDIX B. Compressed air was used for the purpose of providing air bubbles for scouring the membrane surface during the study. The flow rate of the air was measured using the digital readout on the electronic flow meter and the desired flow rate (5 or 15 LPM) for experimental purposes was achieved using the flow modulator. The air was filtered using the HEPA-VENT ${ }^{\mathrm{TN}}$ at the source to avoid any contamination of the flow line and air supplied to the reactor tank and also to preserve the working condition of the flow meter.

While the membrane module had a built in air scouring system, it was observed that the air bubbles created by the system did not completely scour the surface of all membrane fibers. More specifically, the visible surface membrane fibers on the module did not seem to be scoured due to the localized nature of air bubbles provided by the built in scouring system. To ensure that the entire effective membrane surface was scoured by the air during experiments, a flexible Boyu air curtain diffuser was set up around the base of the membrane strands to provide coarse bubbles for the scouring of the visible surface of the membrane strands. Air was provided to the ZW-1 built in air scouring system and the Boyu air diffuser simultaneously during experiments such that the total air flow provided for air scouring was equal to the desired air flow for the experiment performed. 
A valve was installed prior to the Boyu air diffuser to balance the flow supplied by both air systems.

The Feed flow line made use of a $50 \mathrm{~L}$ Nalgene heavy duty cylindrical tank as a feed tank to store the feed water. A Cole-Parmer submersible pump (max flow of 3.5 GPM) was initially used to continuously mix and maintain the homogeneity of the feed water in the tank but the heat generated by the pump increased the water temperature over time. Intermittent mixing resolved the temperature issue however subsequent to this, an oil leak from the submersible pump occurred. As such mixing was switched to the $\mathrm{VWR}^{\mathrm{TM}}$ overhead mechanical stirrer (Power Max Elite Dual Speed Meter) and a 3-blade propeller to continuously mix the feed water and maintain homogeneity. Feed water was continuously supplied from the feed tank to a $3 \mathrm{~L}$ plexiglass cylindrical reactor tank using a Masterflex peristaltic pump (model \# 7024-20) during experiments. Any overflow from the reactor tank was directed back into the feed tank. Cole-Parmer C-flex tubing, Masterflex high performance precision tubing and Masterflex polypropylene straight connectors were used in the Feed flow line. A detailed schematic of the Feed flow line can be seen in APPENDIX B.

The Permeate flow line was the most complex line of the three flow lines and incorporated an air trap/line flushing system. Suction required to produce permeate from the ZW-1 module was provided by a Masterflex L/S digital peristaltic pump system with an Easy-Load 3 pump head for high-performance precision tubing. The use of the digital pump allowed for the automation of the flow rate and flow direction of permeate and thus was an integral component in the bench scale apparatus. Permeate was directed from the membrane module to a $1 \mathrm{~L}$ backwash tank during forward filtration periods and vice versa 
during backwash periods. Over flow from the backwash tank was directed back to the feed tank. A Cole-Parmer high accuracy ( $+/-0.25 \%$ full scale) differential pressure transducer was installed between the membrane module and digital pump and was used to measure the transmembrane pressure during experiments.

An air trap/line flushing system was installed between the transducer and digital pump. The main purpose of the trap was to catch air bubbles and prevent the build-up of bubbles in the permeate flow line. The air trap was made up of a $100 \mathrm{~mL}$ glass tank with two openings installed above the line of permeate flow. While one of the openings was connected to the permeate line, the other opening was capped with a Teflon pierce-able cap. To activate the air trap, air in the tank was sucked out using a syringe through the pierce-able cap during the course of trial experiments thus creating a vacuum in the tank. As a result permeate flowed into the tank filling up the tank. The air trap then relied on gravity as permeate in the tank displaced air bubbles as they passed the entrance to the tank. The setup of the air trap along with the setup of the entire permeate flow line can be seen in APPENDIX B. While the air trap worked well in trapping air bubbles during experiments it was found that the fluctuating pressures within the air trap affected the transmembrane pressure readings during the experiments. Thus the use of the tank as an air trap system was discontinued. However, a valve was placed between the tank and permeate flow line and the tank was used to aid in flushing the permeate line during the cleaning of the apparatus between experiments.

The pressure transducers and flow meters used in both experimental setups were connected to a computer where data from these instruments were recorded using a data acquisition program Operation Flux 2.0 written specifically for the recording of data for 
the study. Operation Flux 2.0 allowed the recording of data received from the flow meters and pressure transducers over time intervals programmed by the user for the various experimental flow operation regimes employed in the study. The operations of the Operation Flux 2.0 included the categorization of data received and the storage of data in the form of a simple text file to be later analyzed by the user. The code for the program is included in APPENDIX $\mathrm{C}$ of this report.

While the electronic flow meter by Differential Pressure Technologies was connected directly to the computer via a USB to serial convertor, the electronic flow meter by Aalborg and both high accuracy pressure transducers were connected to the computer via the ADAM-4019+ universal analog input module and the ADAM-4561 isolate USB to RS-232/422/485 converter by Advantech. The wiring diagram representing the circuitry connecting the instruments to the ADAM modules can be seen in APPENDIX D. The Masterflex L/S digital peristaltic pumps used in the permeate flow line were also connected to the computer using a USB to serial converter. The permeate flow rate and direction was controlled and automated using the Masterflex linkable instrument control software WinLIN. All forward filtration, backwash and relaxation intervals and durations used in the study were programmed and controlled using WinLIN. Common problems faced and trouble shooting for WinLIN and Operation Flux 2.0 are included in APPENDIX E.

\subsubsection{Simulated Test Water}

Simulated river water was used as the test water for all experiments. This particularly high fouling water was made up of distilled water, an organic mix of Sigma 
Aldrich humic acid ( $3 \mathrm{mg} / \mathrm{L}$ TOC) and Sigma Aldrich cellulose $(3 \mathrm{mg} / \mathrm{L}$ TOC), an inorganic component in kaolin clay (20 NTU) and hardness chemicals sodium bicarbonate $\left(75 \mathrm{mg} / \mathrm{L}\right.$ as $\left.\mathrm{CaCO}_{3}\right)$ and calcium sulphate $\left(75 \mathrm{mg} / \mathrm{L}\right.$ as $\left.\mathrm{CaCO}_{3}\right)$. The main reason for using such a fouling water was to ensure that fouling occurred during experiments. Humic acid known for its high fouling potential (Oschmann et al., 2005) was used to represent the dissolved organic particles in source waters while cellulose (20 $\mu \mathrm{m})$ was used to represent organic particulates in source water.

A humic acid and cellulose organic concentrate was usually prepared 24 hours prior to the start of experimental runs by vigorously mixing humic acid and cellulose in $2 \mathrm{~L}$ of a $50 \mathrm{ppm}$ sodium hydroxide solution $(\mathrm{pH} 10)$. The high $\mathrm{pH}$ used ensured that the solid humic acid dissolved in solution and represented dissolved organics in the test waters. Due to the complex structure of humic acid, the amount of humic acid required to obtain $3 \mathrm{ppm}$ of total organic carbon (TOC) in solution was determined by performing liquid and solid TC analysis using the TOC-V CPN unit and SSM 5000-A unit of a SHIMADZU TOC-VCPH/CPN total organic carbon analyzer respectively. Results from the liquid TOC test of humic acid were found to be higher than that of the solid TC indicating the possibility of contamination in the TOC-V CPN unit. Thus, results from the solid TC test were used to identify the amount of humic acid (shown in APPENDIX F) needed to supply $3 \mathrm{ppm}$ of TOC in the feed solution. In the case of identifying the amount of cellulose to be added in solution, similar liquid TOC and solid TC tests were performed in which results from the liquid TOC tests were 3 fold lower than the solid TC tests. The large discrepancy in results was thought to be due to the in ability of the TOCV CPN unit to maintain the cellulose in suspension during sampling. As a result it was 
found that the liquid TOC analyser was not suitable for measuring the carbon content caused by cellulose in solution and solid TC results were used to identify the amount of cellulose (Shown in APPENDIX F) needed to add 3ppm of TOC in feed solution.

On the day of experimentation the humic acid - cellulose concentrate was added to distilled water along with kaolin clay and the hardness chemicals to form the simulated feed water. $42 \mathrm{~L}$ and $50 \mathrm{~L}$ of test solutions were prepared for each experiment during the Preliminary Study Phase and Main Phase Experiments respectively. The required amounts of kaolin clay and hardness chemicals used to prepare the simulated water can be seen in APPENDIX F. On preparation of the test water, the solution was initially mixed at $700 \mathrm{rpm}$ ( $\mathrm{G}$ value of 324/s) for approximately $1 \mathrm{~min}$ to ensure complete mixing of all ingredients in solution. The test water was then continuously mixed at $350 \mathrm{rpm}$ (G value of 229/s) for the entire duration of an experimental run.

\subsection{Experimental Design}

A number of two level factorial experimental designs were employed under this study. In two level factorial experimental designs, combinations of ' $k$ ' independent variables at two levels are tested to determine the effect of the independent variables on a measured dependent variable. A major advantage of performing two way factorial designs is the reduced number of experiments required $\left(2^{k}\right.$ number of combinations) compared to the number of experiments required when individually testing the effect of each factor, factor levels and the sequential combinations on the dependant variable. Another advantage of factorial experimental designs is the identification of the most influential factors (positive or negative influence) effecting the dependent variable. These 
designs also highlight existing interactions between factors thus allowing the determination of symbiotic or synergistic relationships between factors and the effect of these relations on the dependent variable.

Factorial experimental designs are particularly beneficial when investigating numerous factors since a general linear model can be applied to the data providing an exact representation of the data. This linear model can also be used to identify potential highly influential factors as well as eliminate factors and interactions with the least influence. The remaining model can then be used to determine a time based model for the prediction of fouling under the tested conditions over time.

The study was divided into three phases; the Preliminary Study Phase, The Main Phase Experiments and the Follow-Up Experiments. The sequence of phases and the research included in each phase can be seen in Figure 3.2. The following sections describe the various experimental designs and procedures used in the three study phases.

Figure 3.2 - Research performed at the Three Study Phases

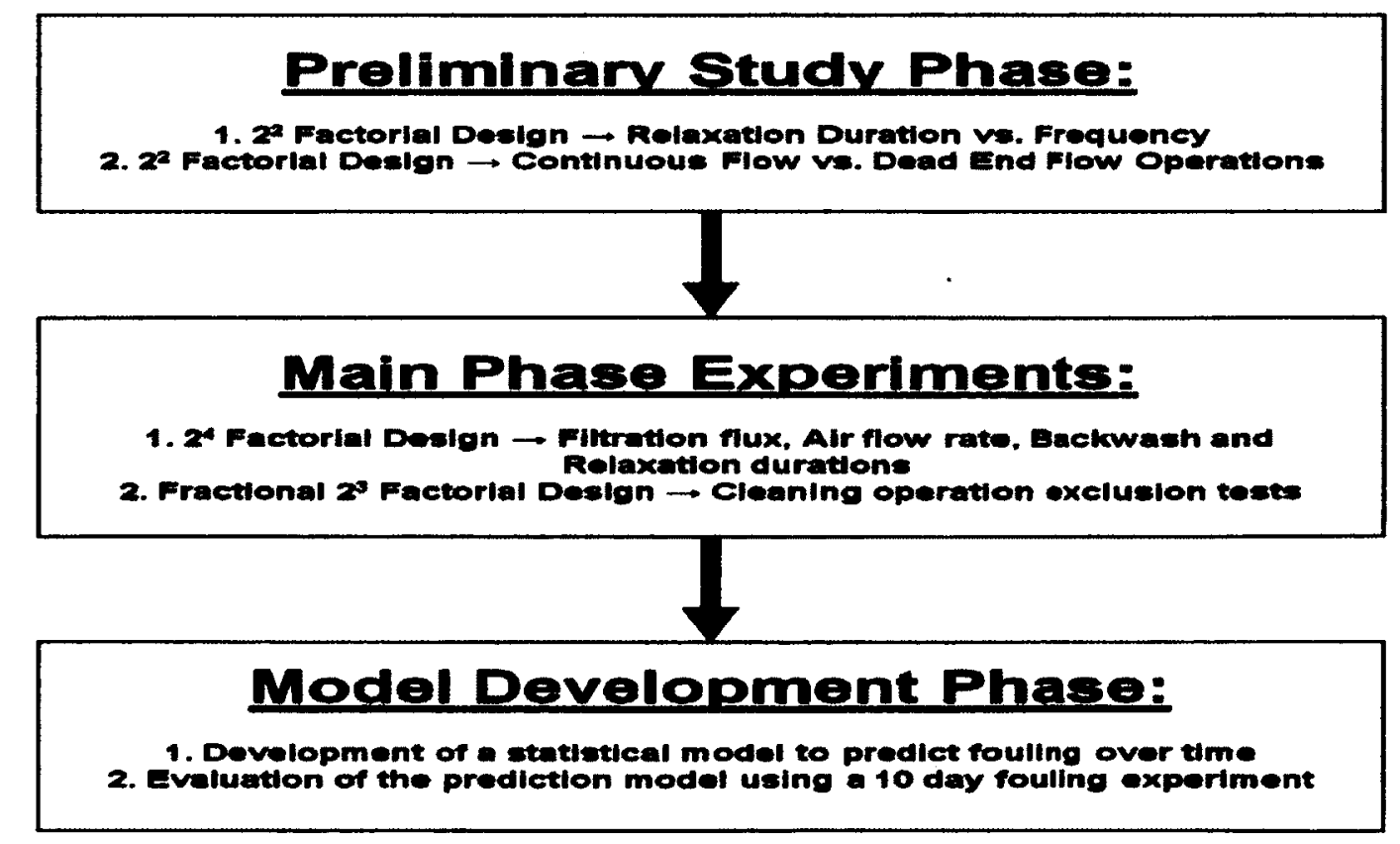




\subsubsection{Preliminary Study Phase}

A number of studies employing a $2^{2}$ factorial design of experiments were completed during the Preliminary Study Phase. These studies investigated two relaxation duration periods ( 5 and 15 minutes) as well as two interval periods ( 2 and 4 hours) between relaxation modes as shown in Table 3.1. The effect of dead-end flow operations and continuous flow operations were also investigated during this phase.

Table 3.1 $-2^{2}$ factorial design used in the Preliminary Study Phase to investigate the effect of relaxation duration and interval on membrane fouling.

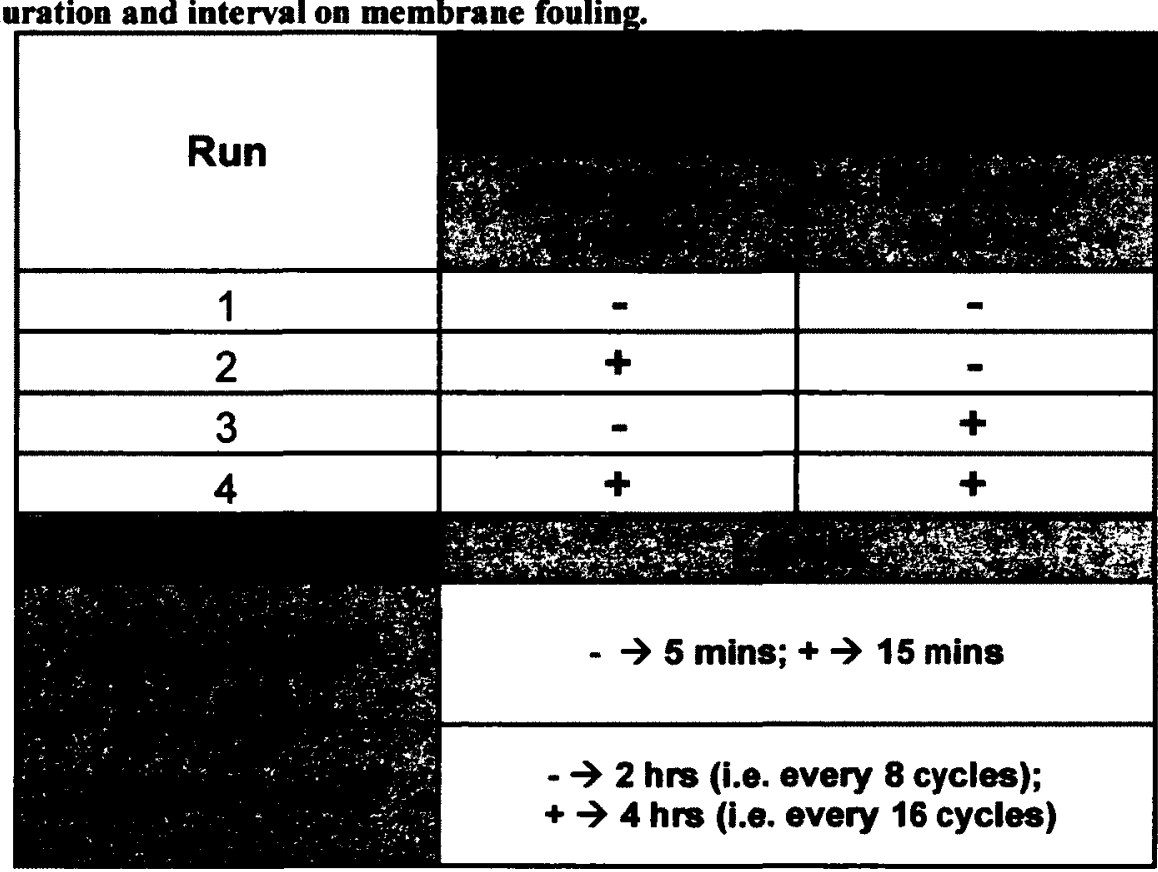

*Note: 1 cycle $=$ forward flow + backwash flow

\subsubsection{Preliminary Study Phase A}

Preliminary Study Phase A, made use of the $2^{2}$ factorial design of experiments (Table 3.1) to investigate the effect of relaxation duration and relaxation frequency on the reduction of fouling in the submerged hollow fiber bench scale apparatus. A dead-end operation in which the filtration flow was the same as the feed flow was employed for 12 
hr. long experiments with constant forward flow, backwash flow and air flow as seen in Table 3.2.

Membrane fouling was measured in terms of transmembrane pressure (TMP). TMP readings and air flow readings were logged every 15 seconds during the filtration and relaxation period and every second during backwash periods throughout each $12 \mathrm{hr}$ experimental run using the data acquisition program Operation Flux 2.0. The feed water used during these experiments was similar to that described in Section 3.1.2 with the exception that $3.36 \mathrm{~g}$ of kaolin clay was used in 42 litres of feed water.

Table 3.2 - Operational parameters used in Preliminary Study Phase A - Relaxation duration vs, Frequency

\begin{tabular}{|l|c|}
\hline Forward flow & $60 \mathrm{ml} / \mathrm{min}(76.6 \mathrm{LMH})$ for $15 \mathrm{mins}$ \\
\hline Backwash flow & $60 \mathrm{ml} / \mathrm{min}(76.6 \mathrm{LMH})$ for $10 \mathrm{secs}$ \\
\hline Air flow rate & $5 \mathrm{LPM}$ throughout experimental run \\
\hline Relaxation & \\
\hline Experiment 1 & Duration $5 \mathrm{mins}$; Interval 2 hrs. (i.e. every 8 cycles) \\
Experiment 2 & Duration $15 \mathrm{mins}$ interval 2 hrs. (i.e. every 8 cycles) \\
Experiment 3 & Duration 5 mins; Interval 4 hrs. (i.e. every 16 cycles) \\
Experiment 4 & Duration 15 mins; Interval 4 hrs. (i.e. every 16 cycles) \\
\hline
\end{tabular}

Note: 1 cycle = forward flow + backwash flow

Samples of the feed and permeate were taken at the beginning and end of each experimental run and tested for $\mathrm{pH}$, turbidity and $\mathrm{COD}$. These water quality parameters were measured to identify any significant changes in water quality over each $12 \mathrm{hr}$. experimental run that might be of concern for future experiments. Water quality analysis initially included liquid TOC measurements, however, as previously mentioned, due to the inefficiency of the TOC analyzer when measuring cellulose in solution compounded 
by the inefficiency due to the presence of suspended solids in solution, TOC measurements were abandoned. The $\mathrm{pH}$, turbidity and $\mathrm{COD}$ for the Preliminary Phase A experiments and all proceeding experiments were measured using an Oakton $\mathrm{pH}$ meter (pH 11 series), an Orion $\mathrm{AQ} 3010$ turbidimeter and $\mathrm{HACH}$ low range COD digestion vials (Method 8000) respectively.

\subsubsection{Preliminary Study Phase B}

Preliminary Study Phase B investigated the effect of dead-end operation and continuous flow operation on the fouling of hollow fiber membranes. Two sets of five experiments were employed during this investigation. Each set of experiments involved the $2^{2}$ factorial design of experiment investigating relaxation duration vs. relaxation frequency (Table 3.1) as well as a fifth experiment investigating the effect of physical cleaning regimes without relaxation on membrane fouling. The first set of experiments in this phase employed dead-end operation whereas the second set of experiments employed continuous flow operation. During dead-end operations the filtration flow from the reactor tank was the same as the feed flow into the reactor tank. Continuous flow operations were set such that an $80 \%$ recovery was obtained while the feed flow into the reactor tank was higher than the filtration flow from the reactor tank. Based on the results of Preliminary Study Phase A, a few changes were made to the operational parameters, water quality parameters and also the apparatus setup.

With regards to the modification of the apparatus setup, most of the permeate line initially made up of high performance yet soft tubing was replaced by hard FEP tubing to

minimize the effects of tubing failure on TMP readings and permeate flow.. In terms of 
water quality parameters, only $1.6 \mathrm{~g}$ of kaolin clay was used in $42 \mathrm{~L}$ of feed water to provide a turbidity value of 20 NTU without settling. A $30 \mathrm{ml} / \mathrm{min}$ flow was used for forward and backwash permeate flow for both experimental sets which is much closer to the suggested maximum membrane capability of $25 \mathrm{ml} / \mathrm{min}$ than the flow used in Preliminary Study Phase A experiments. The operational parameters and relaxation mode regimes for experiments employing dead-end operation and continuous flow operation can be seen in Table 3.3.

Table 3.3 - Operational parameters used in Preliminary Study Phase B - Dead-end vs. Continuous Flow Operations

\begin{tabular}{|c|c|}
\hline \multicolumn{2}{|l|}{ Permeate } \\
\hline Forward flow & $30 \mathrm{ml} / \mathrm{min}(38.3 \mathrm{LMH})$ for $15 \mathrm{mins}$ \\
\hline Backwash flow & $30 \mathrm{ml} / \mathrm{min}(38.3 \mathrm{LMH})$ for 10 secs \\
\hline \multicolumn{2}{|l|}{ Feed } \\
\hline Forward flow & $37 \mathrm{ml} / \mathrm{min}$ for continuous flow, $30 \mathrm{ml} / \mathrm{min}$ for dead-end \\
\hline \multicolumn{2}{|l|}{ Air Scouring } \\
\hline Air flow rate & 5 LPM throughout experimental run \\
\hline \multicolumn{2}{|l|}{ Relaxation } \\
\hline Experiment 1 & Duration 5 mins; Interval 2 hrs (i.e. every 8 cycles) \\
\hline Experiment 2 & Duration 15 mins; Interval 2 hrs (i.e. every 8 cycles) \\
\hline Experiment 3 & Duration 5 mins; Interval 4 hrs (i.e. every 16 cycles) \\
\hline Experiment 4 & Duration 15 mins; Interval 4 hrs (i.e. every 16 cycles) \\
\hline Experiment 5 (Base) & No relaxation \\
\hline
\end{tabular}

Note: 1 cycle $=$ filtration flow + backwash flow

TMP readings and air flow readings were logged every 15 seconds during the forward flow and relaxation period and every second during backwash periods for each Phase 2 experimental run. All Preliminary Study Phase B experiments were run for 24 hrs. Samples of the solution in the feed tank, reactor and permeate were taken at the start 
and end of each experiment as well as at the $12 \mathrm{hr}$. mark of each run. These samples were tested for $\mathrm{pH}$, turbidity and COD to identify changes in water quality over the $24 \mathrm{hr}$. run. Temperature readings of the solution in the reactor tank were also taken mostly at the start and end of experimental runs.

\subsubsection{Main Phase Experiments}

Two sets of experiments were performed under the Main Phase Experiments. A $2^{4}$ factorial design of experiments was employed to investigate the effect of air flow rate, backwash duration, relaxation duration as well as filtration flux on membrane fouling. A fractional $2^{3}$ factorial design of experiment was also performed to study the effect of cleaning operation exclusion on fouling reduction. Findings from the Preliminary Study Phase were taken into account during this phase and thus a continuous flow operation with 80\% recovery was used for all Main Phase Experiments. Sections 3.2.2.1 and 3.2.2.2 further discuss the experimental designs and operational parameters used during these Main Phase Experiments.

\subsubsection{1 $2^{4}$ Factorial Design of Experiments}

A $2^{4}$ factorial design of experiments was employed during the Main Phase Experiments to fulfil the main research objective of investigating the effect of air scouring, backwashing and relaxation as well as filtration flux on the minimization of fouling of a bench scaled submerged hollow fiber membrane system. The 16 experiments belonging to the factorial design shown in Table 3.4 were thus performed in random to analyze the effect of filtration flux (19 and $38 \mathrm{LMH})$, air scour flow rate (5 and $15 \mathrm{LPM}$ ), 
backwash duration (10 and $20 \mathrm{sec}$.) and relaxation duration ( 5 and $15 \mathrm{~min}$.) on fouling. In addition to the 16 planned experiments, a number of the 16 experiments were duplicated to ensure reproducibility of the results as well as confirm results thought to be suspicious. These duplicated experiments included Experiments 2, 6, 10 and 16 from Table 3.4.

Membrane fouling during the $2^{4}$ factorial design of experiments was measured in terms of TMP logged every 15 seconds during filtration and relaxation periods and every second during backwash periods over the 5 day experimental run using Operation Flux 2.0. The feed water used in the experiments was the simulated test water discussed in Section 3.1.2. Water quality assessment of the feed reactor and permeate were monitored for each experimental run to identify any significant changes in water quality that may affect fouling. While $\mathrm{pH}$ and turbidity readings were monitored daily, the COD of the three solutions was monitored at the beginning of the each experiment, after 48 hours and finally at the end of each experiment. The flow rate of the permeate was also monitored at the beginning and end of experiments as well as after 48 hours. Chemical cleaning as described in Section 3.3 was also performed after every 5 day experiment.

A number of membrane modules were employed over the duration of the Main Phase Experiments. Fouled membrane modules were replaced with new virgin membrane modules when chemical cleaning was found to be ineffective in restoring the permeability of the membrane. As a result of the permeability loss, the expected initial productivity of the membrane during the high flux (38 LMH) experiments was not achievable thus requiring the use of a new membrane to maintain the consistency of experimental results. 
Table $3.4-2^{4}$ factorial design used to investigate the effect of filtration hux, air flow rate, backwashing duration and relaxation duration on membrane fouling

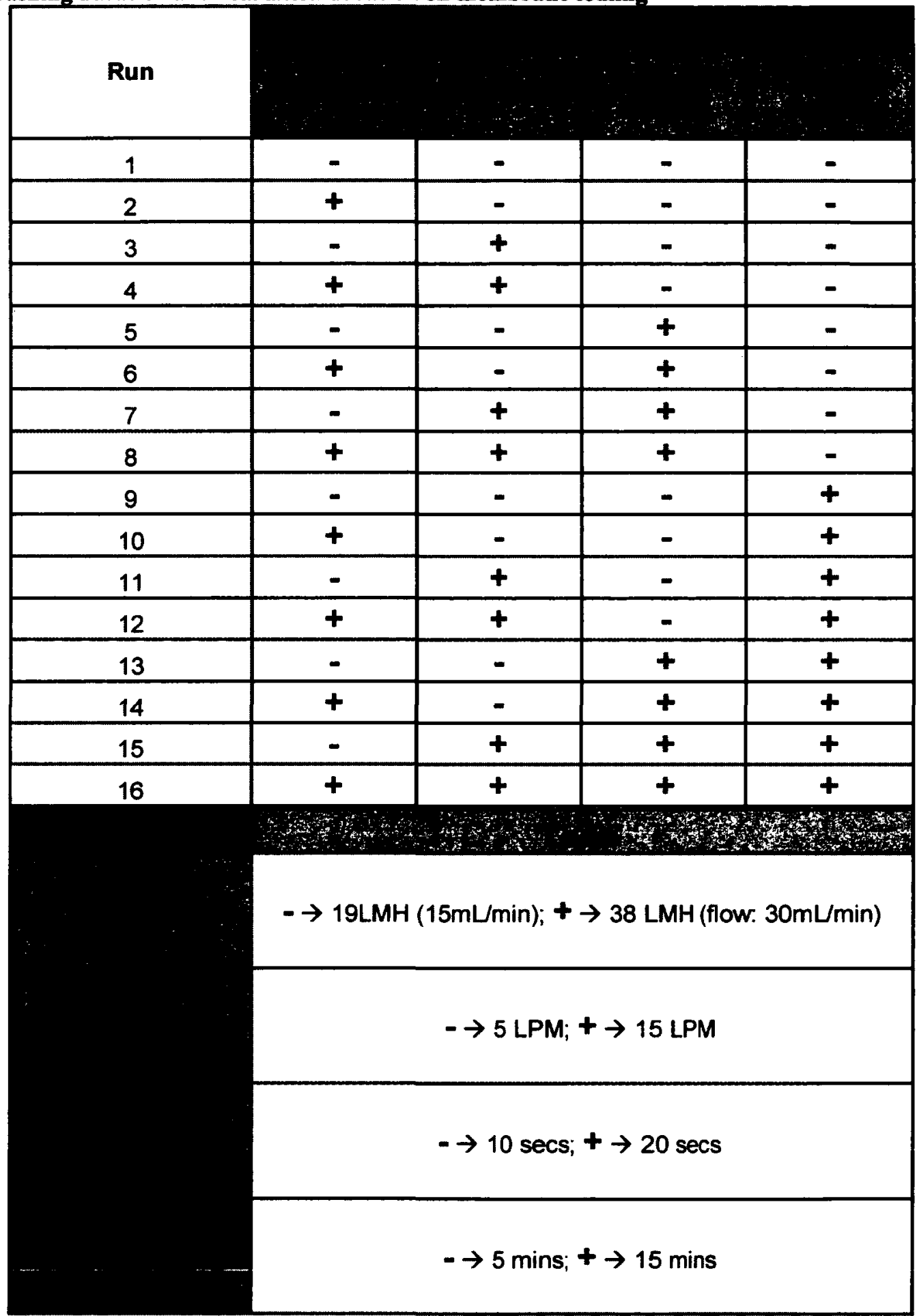

*Note: 1 cycle = filtration flow + backwash flow 


\subsubsection{Cleaning Operation Exclusion Tests}

Cleaning operation exclusion tests made use of a fractional $2^{3}$ factorial design of experiments (seen in Table 3.5) to investigate the effect of individual exclusion of cleaning operations on fouling. These experiments were based on Experiment \# 2 of the $2^{4}$ factorial design due to the high fouling nature of the experiment that was thought to better highlight the effects of operation exclusion on fouling.

Table 3.5 - Fractional factorial design used to investigate the impact of cleaning parameters exclusion on membrane fouling. Filtration flux $\rightarrow 38$ LMH; Filtration duration $\rightarrow 15$ minutes

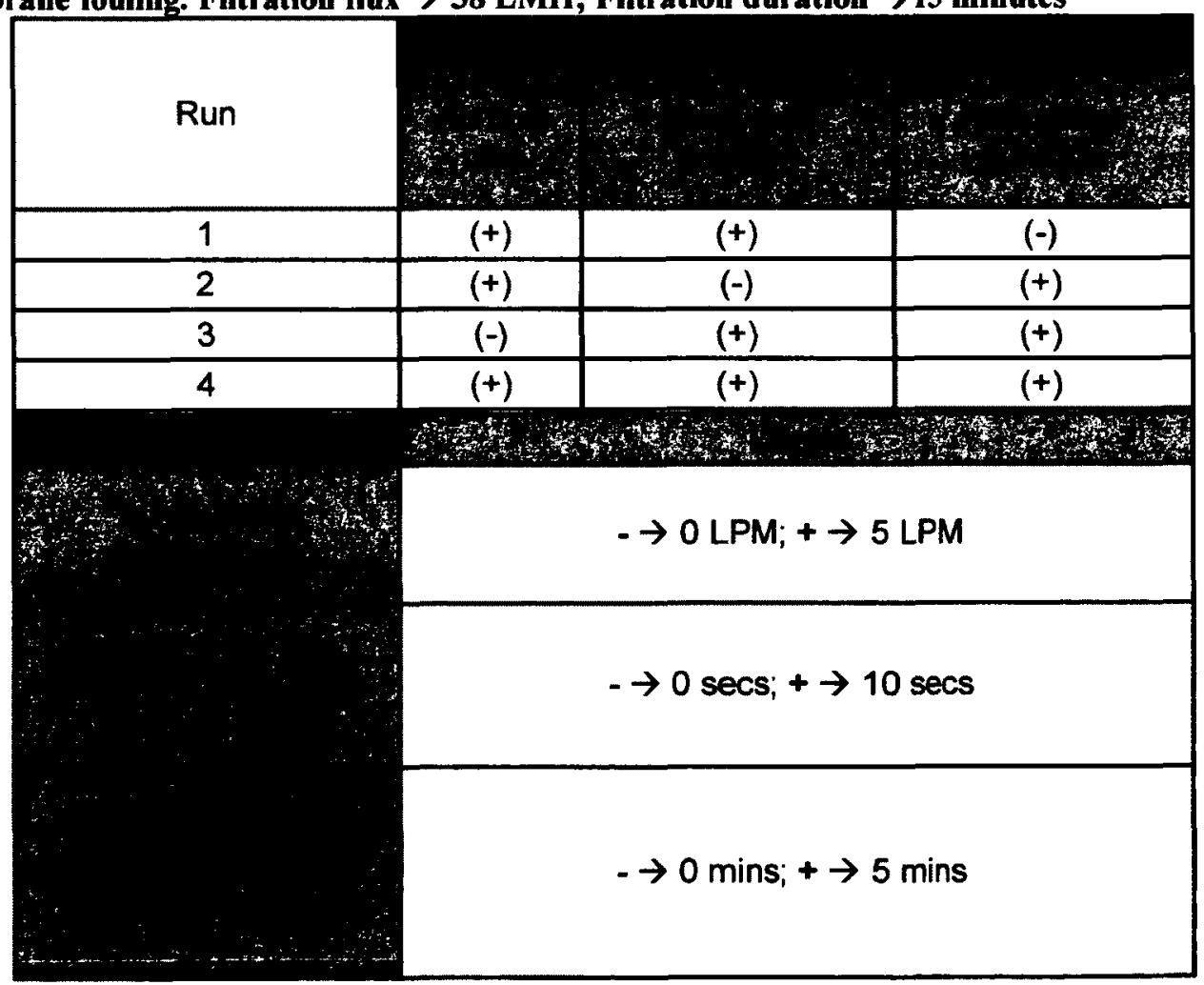

*Note: 1 cycle $=$ filtration flow + backwash flow

Fouling was measured in terms of TMP measured every 15 seconds during filtration and relaxation periods and every second during backwash periods using over the 48 hour experiments using Operation Flux 2.0. The feed water used in these exclusion 
experiments can be seen in Section 3.1.2. Water quality assessment ( $\mathrm{pH}$, turbidity, COD) was performed at the beginning of the experiments as well as at the $24 \mathrm{hr}$ and $48 \mathrm{hr}$ mark. The permeate flow was also measured daily to monitor for losses of productivity due to high fouling. Chemical cleaning was performed at the end of the 48 hour experiments as described in Section 3.3.

\subsection{Cleaning Procedure}

Chemical cleaning of the membranes was provided following every experimental run in attempts to restore the permeability of the membrane before the proceeding experiment. At the end of an experimental run, prior to chemical cleaning, the surface of the membrane was sprayed with warm tap water $\left(<40^{\circ} \mathrm{C}\right)$ to remove any cake layer from the membrane surface. The membrane module was then allowed to soak in warm tap water $\left(<40^{\circ} \mathrm{C}\right)$ for a short duration of $1 \mathrm{hr}$. during which short spurts of periodic backwashing at a flux of $76 \mathrm{LMH}$ were applied to promote the dislodging of foulants from the membrane surface and membrane pores. The TMP was monitored during the periodic backwashes and was not permitted to rise above 0.25 bar.

Following the $1 \mathrm{hr}$. warm water soak, the membrane was allowed to soak in a 200 ppm solution of sodium hypochlorite (Javex 6\%) for a minimum of $18 \mathrm{hrs}$. After $18 \mathrm{hrs}$ the module was soaked in distilled water for a short period of time during which periodic backwashes were continued to further promote the removal of any fouling remnants off the surface of the membrane. If a cloud of foulants was noticed off the surface of the membrane after backwash period, the membrane was put through an additional 5 hour sodium hypochlorite soak. If no foulant cloud was noticed off the surface of the 
membrane, the module was put through a soak in citric acid (Sigma Aldrich) solution for a minimum of 18 hours. At the end of the cirtic acid soak, the membrane was flushed with distilled water and allowed to soak in distilled water till the start of the next scheduled experiment.

The feed tank, reactor tank and backwash tank were all thoroughly washed with warm water and cleansing detergent before every experiment. The tubing used in the feed and permeate flow lines were also cleaned before every experiment. The tubing was first flushed with a $100 \mathrm{ppm}$ sodium hypochlorite solution followed by distilled water. The line flushing tank with the pierce-able cap and a syringe was used to help with the flushing of the permeate flow line. Tubing used in the pump drives were replaced on a biweekly basis to ensure optimal performance of the tubing when creating the required suction for the feed flow and permeate flow.

\subsection{TMP/ TMP curves}

Fouling during the Preliminary Study Phase and Main Phase Experiments was measured in terms of TMP (mbar). The change in fouling for each experiment was calculated as the difference between the lowest TMP observed during the first and final 15 minutes of each experiment. For Main Phase Experiments that were duplicated, TMP readings used in the analysis of the factorial design results were the averages of the duplicated experiments.

TMP graphs were vital for the evaluation of cleaning operational behavior during the experiments. For the purposes of better comparison of TMP trends over time, 
backwash and relaxation data points were filtered from the data using a filtering macro in Excel written specifically for the purpose of the project. The code for the macro can be seen in APPENDIX G.

\subsection{Data analysis}

A number of factorial designs were employed under this study to investigate the effect of relaxation parameters on reducing fouling as well as investigate the fouling reducing effects of relaxation, backwashing, air scouring and filtration flux when included in a cleaning operational regime promoting fouling reduction. Factorial designs have proved to be very economic and effective designs of experiments when the effect of a number of independent variables on a dependant variable is sought after (Mukerjee and $\mathrm{Wu}, 2006$ ). In factorial designs, various levels of factors (independent variables) are tested in combination while monitoring the effect of the treatment on a specific response (dependent variable).

The number of treatment combinations $\left(\mathrm{m}^{\mathrm{k}}\right)$ under a factorial design is dependent on the number of factors $(\mathrm{k})$ and factor levels $(\mathrm{m})$. Where $\mathrm{k}$ represents the independent variable (i.e. flow rate) while $\mathrm{m}$ represents the values we perturb the variable against (i.e. $15 \mathrm{~mL} / \mathrm{min}$ and $30 \mathrm{~mL} / \mathrm{min}$ ). For example, a full factorial design involving 2 factors, each having 2 factor levels would have a total of $2^{2}=4$ combinations while a design with 4 factors and 2 factor levels would have a total of $2^{4}=16$ combinations. Fractional factorial designs are a popular alternative to full factorial designs when running full factorial designs are not practical (Mukerjee and Wu, 2006). Fractional factorial designs involve selected subsets of full factorial designs and the number of treatment combinations may 
vary depending on the amount of data required. Half-fractional factorial designs in which half of the combinations of a full factorial design are selected are usually denoted as $\mathrm{m}^{\mathrm{k}-1}$ designs (Burn, 2010). Factorial designs with 2 factor levels are known as two-level factorial designs and usually incorporate a high $(+)$ and low (-) value of a factor as factor levels. Two-level factorial designs were used for all factorial experiment designs used in this study.

Results from factorial design experiments can be analysed using linear regression or the analysis of variance (ANOVA) approach to linear regression. While similar, both methods complement each other and thus were used when analysing the results from the various two-level factorial design experiments performed in this study. The following sections inform on the factorial ANOVA and regression methods used during data analysis in this study.

\subsubsection{Factorial ANOVA}

Analysis of variance (ANOVA) is a beneficial preliminary tool used to analyze results of factorial experiments as it allows the investigation of the effect of factors on response variables while identifying the presence of interaction effects of factors on the response. Furthermore, the ANOVA model allows for the easy setup of interaction plots which are extremely useful when assessing the existence of interactions and the importance of main and interaction effects. Table 3.6 shows the ANOVA model for a simple $2^{2}$ factorial design with factors $A$ and $B$ having levels $i$ and $j$ respectively. This model was used to analyze the results of the $2^{2}$ factorial design experiments used in the Preliminary Study Phase. 
Table 3.6 - ANOVA model for $\mathbf{2} 2^{2}$ factorial design.

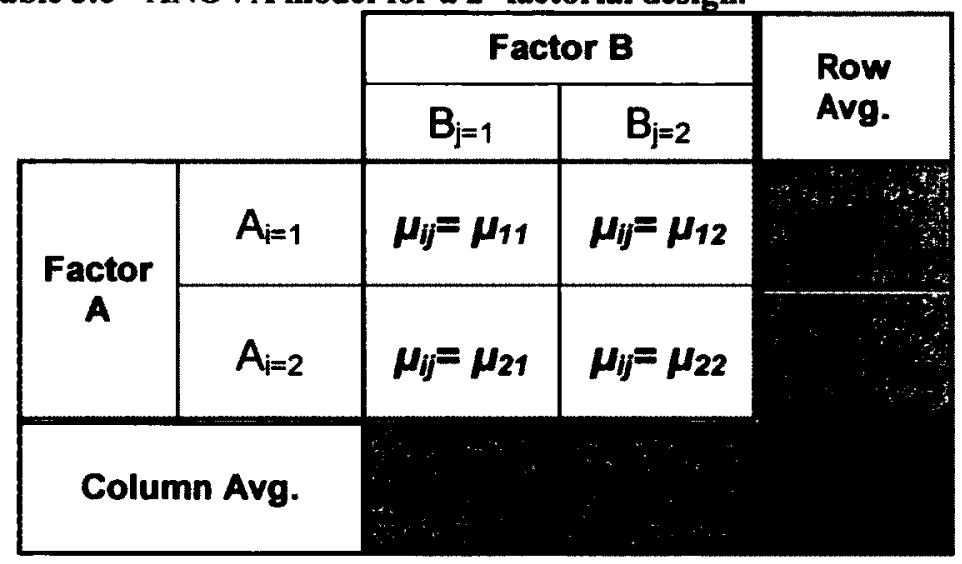

In the model, the mean response for a treatment combination is denoted as ' $\boldsymbol{\mu}_{\mathrm{ij}}$ ' while factor level means are denoted as ' $\boldsymbol{\mu}_{\mathbf{i}}$ ' and ' $\boldsymbol{\mu}_{\mathrm{j}}$ ' for factor A and B respectively. The main effects of factors were obtained by comparing the factor level means with the overall mean ' $\boldsymbol{\mu}$. .. . These main effects of factor $\mathrm{A}$ and $\mathrm{B}$, denoted as ' $\boldsymbol{\alpha}$ ' and ' $\boldsymbol{\beta}$ ' respectively, can be seen in Equations 3.1 and 3.2. The importance of the effect of one factor over the other was determined by comparing the magnitude of the factor effects, the greater effect being of more importance. While factor effects closer to 0 may imply no change from the overall mean and infer minimal effect by the factor level on the response, these main effects cannot be taken at face value as interaction effects of the factors on the response may exist.

$$
\begin{aligned}
& \alpha_{i}=\mu_{i .}-\mu_{. .} \quad\left(\text { Such that } \sum_{i} \alpha_{i}=0\right) \\
& \beta_{j}=\mu_{. j}-\mu_{. .} \quad\left(\text { Such that } \sum_{j} \beta_{j}=0\right)
\end{aligned}
$$

(Equation 3.1)

(Equation 3.2) 
Main factor effects are said to be additive such that the mean response for a certain treatment can be calculated by adding the respective factor effects to the overall mean as portrayed in Equation 3.3. The presence of interactions was thus determined by performing the additive test. If the mean response could not be expressed as Equation 3.3, interaction effects were said to be present. Interaction effects ' $\alpha \beta_{i j}$ ' were then calculated using Equation 3.4.

$$
\begin{aligned}
& \mu_{i j}=\mu_{. .}+\alpha_{i}+\beta_{j} \\
& \alpha \beta_{i j}=\mu_{i j}-\left(\alpha_{i}+\beta_{j}+\mu_{. .}\right)
\end{aligned}
$$

While Equation 3.4 provided the interaction effects, a graphical representation of the interactions in the form of interaction plots was necessary to help visually identify the importance of the interactions. Figure $3.3 \mathrm{a}, \mathrm{b}$ and $\mathrm{c}$ presents interaction plots for $2^{2}$ factorial designs involving factors $\mathrm{A}$ and $\mathrm{B}$, each with a low and high level and a response. As can be seen, the X-axis in each plot contains the two levels of factor $\mathrm{A}$ while the Y-axis contains the response value (for these experiments TMP was used as the response variable). The points corresponding to each level of factor B are connected by a straight line thus the two levels for factor B are included in the plot as separate lines. Each of the three plots provides a different scenario to aid in future interpretation of interaction plots in Chapter 4 and 5. 
Figure 3.3 - Interaction plots: a) no interaction; b) antagonistic interaction; c) synergistic interaction (adapted from Kutner et al. (2005))
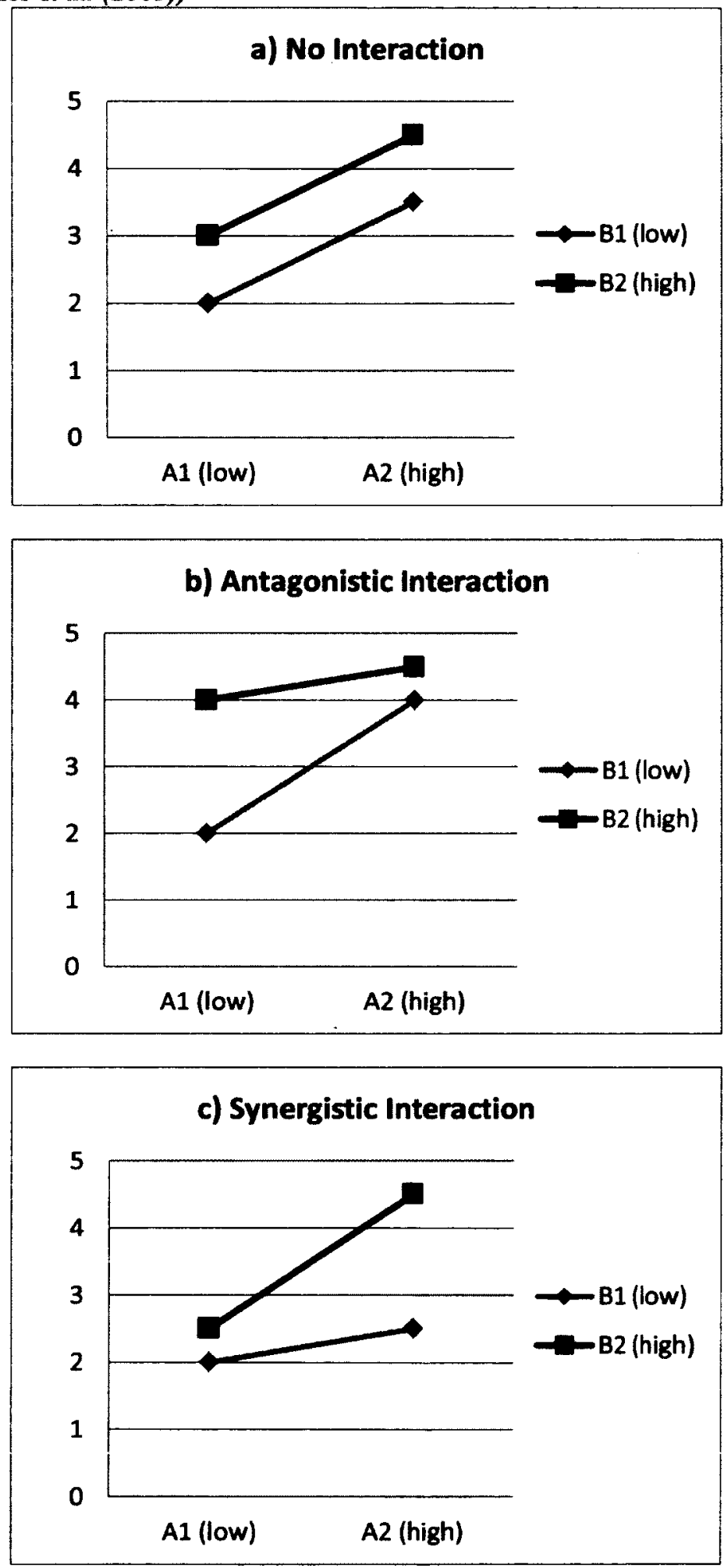
The interaction plot in Figure 3.3a presents a scenario where no interaction exists. While both factor A and factor B have an effect on the response variable, since both lines are parallel, neither factor is dependent on the other. The scenario in Figure $3.3 \mathrm{~b}$ represents the presence of an antagonistic interaction or interference in which an increase of factor B results in a smaller change in response i.e. factor B interferes with the effect of factor A on the response. The opposite scenario is presented in Figure 3.3c where an increase in factor B along with an increase in factor A results in a large increase in the response. Thus factor $\mathrm{A}$ and $\mathrm{B}$ interact synergistically resulting in the increase of the response.

An idea of the importance of existing interactions was also obtained from interaction plots. Interaction plots in which the slopes of the lines are different yet the lines are closely parallel were considered as unimportant interactions while those in which the lines were far from parallel were considered as important interactions. The importance of interactions was further analyzed by testing the nature of main effects (additive, multiplicative etc.). Based on the nature of the main effects, necessary transformations of mean responses (logarithmic, square root etc.) were performed. Interaction plots of the transformed response identified the unimportance of the interaction by removing the interactions effects from the plot. If the interaction effects still existed after transformation then these effects were considered as important effects and were interpreted with care prior to making any conclusions about the main effects.

Similar to the ANOVA model for a $2^{2}$ factorial design presented in Table 3.6, an ANOVA model for a $2^{4}$ factorial design was used for the preliminary analysis of the Main Phase Experiments. The model for the $2^{4}$ factorial design can be seen in 
APPENDIX $H$ while the various equations for the main effects $\left(\boldsymbol{\alpha}_{\mathbf{i}}, \boldsymbol{\beta}_{j}, \mathbf{Y}_{\mathbf{k}}, \boldsymbol{\delta}_{\mathbf{l}}\right)$, two factor

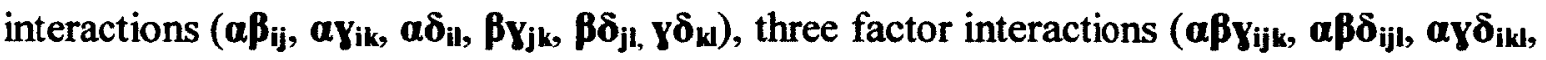
$\left.\beta y \delta_{j k l}\right)$ and four factor interaction $\left(\alpha \beta y \delta_{i j k s}\right)$ can be seen in APPENDIX I. While the ANOVA model was used to set up interaction plots and identify interactions, linear regression was used to identify important effects and interaction effects for the analysis of Main Phase Experiments. It should be noted that since the ANOVA model is a linear model, all main factor effects and interaction effects calculated using the equations in APPENDIX I were identical to the regression coefficients estimated during the determination of the general linear model representing a $2^{4}$ factorial design via linear regression.

\subsubsection{General Linear Modelling}

General Linear Modelling was used mainly for the analysis of the 5 day TMP response data from the $2^{4}$ factorial design experiments used in the Main Phase experiments. The $2^{4}$ factorial design of experiments was represented using the general linear model presented in Equation 3.5. In this model, $\boldsymbol{\beta}$ parameters represent the regression coefficients while $\mathbf{x}_{1}, \mathbf{x}_{2}, \mathbf{x}_{3}$ and $\mathbf{x}_{4}$ represent the four factors with values of +1 and -1 for the high and low levels respectively.

$$
\begin{aligned}
& E(Y)=\beta_{o}+\beta_{1} x_{1}+\beta_{2} x_{2}+\beta_{3} x_{3}+\beta_{4} x_{4} \\
& +\beta_{12} x_{1} x_{2}+\beta_{13} x_{1} x_{3}+\beta_{14} x_{1} x_{4}+\beta_{23} x_{2} x_{3}+\beta_{24} x_{2} x_{4}+\beta_{34} x_{3} x_{4} \\
& +\beta_{123} x_{1} x_{2} x_{3}+\beta_{124} x_{1} x_{2} x_{4}+\beta_{134} x_{1} x_{3} x_{4}+\beta_{234} x_{2} x_{3} x_{4} \\
& +\beta_{1234} x_{1} x_{2} x_{3} x_{4}
\end{aligned}
$$


Least square estimation (adapted from McLean and Burn (2010)) was used to estimate the regression coefficients for Equation 3.5. As previously mentioned these coefficients are the same as the factor and interaction effects obtained using the ANOVA model as presented in APPENDIX I. The least square estimation of the 16 regression coefficients $(\boldsymbol{\beta})$ was performed by matrix algebra using Equation 3.6.

$\beta^{\wedge}=\left(X^{T} X\right)^{-1} X^{T} Y$

(Equation 3.6)

where:

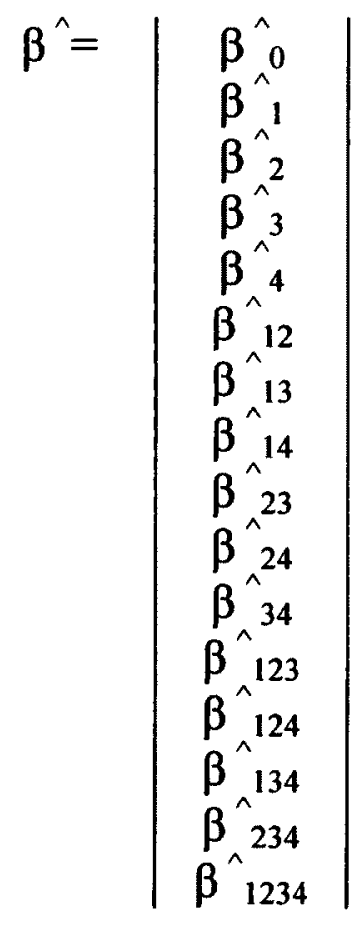




$$
Y=\left|\begin{array}{c}
Y_{-1,-1,-1,-1} \\
Y_{1,-1,-1,-1} \\
Y_{-1,1,-1,-1} \\
Y_{1,1,-1,-1} \\
Y_{-1,-1,1,-1} \\
Y_{1,-1,1,-1} \\
Y_{-1,1,1,-1} \\
Y_{1,1,1,-1} \\
Y_{-1,-1,-1,1} \\
Y_{1,-1,-1,1} \\
Y_{-1,1,-1,1} \\
Y_{1,1,-1,1} \\
Y_{-1,-1,1,1} \\
Y_{1,-1,1,1} \\
Y_{-1,1,1,1} \\
Y_{1,1,1,1}
\end{array}\right|
$$

and

$$
\mathrm{X}=\left|\begin{array}{cccccccccccccccc}
1 & -1 & -1 & -1 & -1 & 1 & 1 & 1 & 1 & 1 & 1 & -1 & -1 & -1 & -1 & 1 \\
1 & 1 & -1 & -1 & -1 & -1 & -1 & -1 & 1 & 1 & 1 & 1 & 1 & 1 & -1 & -1 \\
1 & -1 & 1 & -1 & -1 & -1 & 1 & 1 & -1 & -1 & 1 & 1 & 1 & -1 & 1 & -1 \\
1 & 1 & 1 & -1 & -1 & 1 & -1 & -1 & -1 & -1 & 1 & -1 & -1 & 1 & 1 & 1 \\
1 & -1 & -1 & 1 & -1 & 1 & -1 & 1 & -1 & 1 & -1 & 1 & -1 & 1 & 1 & -1 \\
1 & 1 & -1 & 1 & -1 & -1 & 1 & -1 & -1 & 1 & -1 & -1 & 1 & -1 & 1 & 1 \\
1 & -1 & 1 & 1 & -1 & -1 & -1 & 1 & 1 & -1 & -1 & -1 & 1 & 1 & -1 & 1 \\
1 & 1 & 1 & 1 & -1 & 1 & 1 & -1 & 1 & -1 & -1 & 1 & -1 & -1 & -1 & -1 \\
1 & -1 & -1 & -1 & 1 & 1 & 1 & -1 & 1 & -1 & -1 & -1 & 1 & 1 & 1 & -1 \\
1 & 1 & -1 & -1 & 1 & -1 & -1 & 1 & 1 & -1 & -1 & 1 & -1 & -1 & 1 & 1 \\
1 & -1 & 1 & -1 & 1 & -1 & 1 & -1 & -1 & 1 & -1 & 1 & -1 & 1 & -1 & 1 \\
1 & 1 & 1 & -1 & 1 & 1 & -1 & 1 & -1 & 1 & -1 & -1 & 1 & -1 & -1 & -1 \\
1 & -1 & -1 & 1 & 1 & 1 & -1 & -1 & -1 & -1 & 1 & 1 & 1 & -1 & -1 & 1 \\
1 & 1 & -1 & 1 & 1 & -1 & 1 & 1 & -1 & -1 & 1 & -1 & -1 & 1 & -1 & -1 \\
1 & -1 & 1 & 1 & 1 & -1 & -1 & -1 & 1 & 1 & 1 & -1 & -1 & -1 & 1 & -1 \\
1 & 1 & 1 & 1 & 1 & 1 & 1 & 1 & 1 & 1 & 1 & 1 & 1 & 1 & 1 & 1
\end{array}\right|
$$

The regression coefficients were then inserted into the Original GLM (Equation

3.5) representing the data from the main phase experiments to produce the predicted 
response $\mathrm{Y}^{\wedge}$ (Equation 3.7). Since 16 parameters were estimated using responses for 16 treatment combinations, the response data fit perfectly to the model thus resulting in the Sum of Squares of Residuals (SSR) equal to zero. Equation 3.8 presents the calculation for the residual ' $e$ ' used in the calculation of the SSR for each treatment response.

$$
\begin{aligned}
& Y^{\wedge}=\hat{\beta}_{0}+\beta_{1}^{\wedge} x_{1}+\hat{\beta}_{2} x_{2}+\hat{\beta}_{3} \mathrm{X}_{3}+\beta_{{ }_{4} \mathrm{X}_{4}}
\end{aligned}
$$

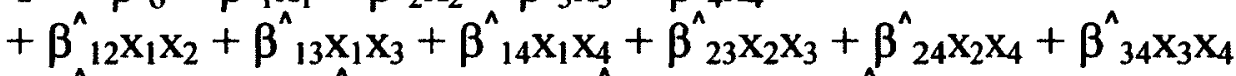

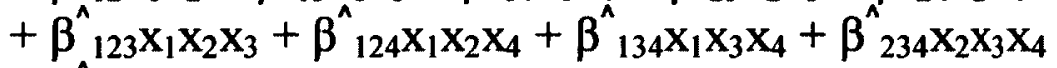

$$
\begin{aligned}
& +\beta^{\wedge}{ }_{1234} \mathrm{X}_{1} \mathrm{X}_{2} \mathrm{X}_{3} \mathrm{X}_{4} \\
& \text { (Equation 3.7) } \\
& e=Y-\left(Y^{\wedge}\right)
\end{aligned}
$$

While the model was an exact representation of the response data, the main model was simplified by only including parameters representing main or interaction effects that had a significant influence on fouling. Two methods of identifying strong main effects and interactions were used during the simplification of the main model. Firstly, single regression coefficient exclusion was performed during which a regression coefficient was excluded from the model and the effect of the exclusion on the SSR was assessed. Parameters were considered unimportant if the SSR was not effected i.e. a value close to 0 was obtained compared to parameters with significantly large SSR which were identified as important. Once unimportant parameters were identified, they were grouped together and combinations of the unimportant parameters were excluded from the main model while monitoring the SSR. The final product from this exclusion test was a candidate model representing the data set from the $2^{4}$ factorial design of experiments. 
While basic comparison of the SSR was able to identify obvious important and unimportant effects due to the magnitude of the SSR compared to that of the original GLM, a more statistical identification of important effects was needed to improve the validity of the model. Forward Stepwise regression was thus performed to statistically verify the important effects identified by basic comparison of SSR.

\subsubsection{Forward Stepwise Regression}

The statistical software JMP 9.0 by SAS was used to perform forward stepwise regression to identify effects that significantly contributed to fouling. The forward stepwise regression model developed a sequence of regression models during which ' $x$ ' variables were either added or deleted from the model at each step of the sequence. The addition and deletion from the model was based on the sum of the squares of the residuals and used the $\mathrm{F}^{*}$ statistic to determine if addition or deletion of the variable from the model resulted in a significant change in the Sum of Squares of Residuals. The $\alpha$-value used to add or delete an ' $\mathrm{x}$ ' variable was 0.10 and 0.15 respectively. While stepwise regression provided the 'best model' possible, the model obtained using stepwise regression was treated as another candidate model for the prediction of fouling over time.

\subsubsection{Prediction Model Development}

One of the objectives of the research study was to use the developed statistical model representing the $2^{4}$ factorial design of experiments to predict the fouling over time when operating any of the cleaning operation regimes used in the Main Phase 
Experiments. To make the GLM time dependent, regression coefficients for the model were estimated for TMP responses recorded at the end of each day of the 5 day Main Study Phase Experiments. The change in regression coefficients over time was then plotted and curves (linear, logarithmic and exponential) best representing the changes for each regression coefficient were obtained. Equations for the curves were inserted into the Original GLM to produce Preliminary Prediction Models for the prediction of fouling. These preliminary models were reduced based on the regression coefficients included in the candidate models obtained during SSR comparison and stepwise regression. The resulting Simplified Prediction Models (SPM) were then evaluated by comparing fouling predicted by the SPM with the actual fouling observed during a 10 day experimental run of Experiment 16 in the Main Phase Experiments. The evaluation of the model using other experiments was intended. However, further experimentation could not be performed due to time restrictions for the completion of the project. A more detail explanation of the model development process and evaluation of the model are provided in CHAPTER 6. 


\section{CHAPTER 4 - Preliminary Study Phase Results}

The Preliminary Study Phase experiments examined impacts of relaxation and flow mode on the experimental system. Preliminary Study Phase A compared the effect of relaxation duration versus the interval between relaxation modes on the fouling of the hollow fiber membranes. Relaxation duration refers to the length of time for which the filtration flux is paused during experiments while relaxation interval refers to the time interval between successive relaxation modes. A set of $2^{2}$ factorial design experiments was performed in the Preliminary Phase A experiments to identify the least effective of the two relaxation parameters to be used for the enhancement of the fouling conditions in the Main Phase Experiments fouling study. In the process, relaxation parameters ideal for the minimization of membrane fouling were also identified.

Preliminary Study Phase B experiments compared the impact of dead-end and continuous flow operation of the membrane filtration system on membrane fouling. These 24 hour long experiments were performed to identify the appropriate flow operation suitable for experiments run over longer periods of time. The effect of the inclusion of relaxation versus the exclusion of relaxation from a cleaning regime was also tested in Phase B. This preliminary phase involved two sets of five experiments; one set employed a dead-end flow operation while the second set employed a continuous flow operation. Each set consisted of the four experiments included in a $2^{2}$ factorial design of experiment investigating relaxation duration vs. relaxation interval and a fifth experiment 
investigating the effect of physical cleaning regimes without relaxation on membrane fouling. The Preliminary Study Phase B experiments incorporated changes to operational and water quality parameters described in CHAPTER 3 Section 3.2.1.2 based on findings from Preliminary Study Phase A. Details of the experiments including operational parameters and test water constituents used for the Preliminary Study Phase experiments are provided in CHAPTER 3.

\subsection{Preliminary Phase A Results}

\subsubsection{Relaxation Duration vs. Interval}

Fouling during the Preliminary Study Phase A experiments was measured in terms of transmembrane pressure (TMP) readings logged every 30 seconds during forward flow and relaxation periods and every second during back wash periods for all experiments using the data acquisition program Operation Flux 2.0. The normalized change in TMP over each experimental run in this preliminary study phase can be seen in Figure 4.1.

On viewing Figure 4.1 it can be seen that experiments involving the shorter relaxation duration period of 5 minutes experienced less change in TMP (i.e. less fouling) than the experiments with the longer relaxation duration period of 15 minutes. On comparing the experiments involving the longer relaxation duration of 15 minutes, it can be seen that the interval (i.e. 2 versus 4 hours) between relaxations did not have an effect 
on the change in TMP. Conversely the interval did impact the TMP change at the shorter relaxation duration of 5 minutes. Furthermore, the experiments involving the shorter relaxation duration period and shorter interval between relaxations experienced the least change in TMP overall. These results indicate that relaxation duration has an effect on membrane fouling while the interval of relaxation (i.e. 2 versus 4 hours) may only have an impact on fouling when using shorter relaxation durations (i.e. 5 versus 15 minutes). To further analyze these findings, a two-way ANOVA analysis was performed to identify important main and interaction effects of relaxation duration and interval between relaxations on changes in TMP $(\triangle T M P)$.

Figure 4.1 - Fouling over time during Preliminary Study Phase A experiments (Flux $\rightarrow 76$ LMH)

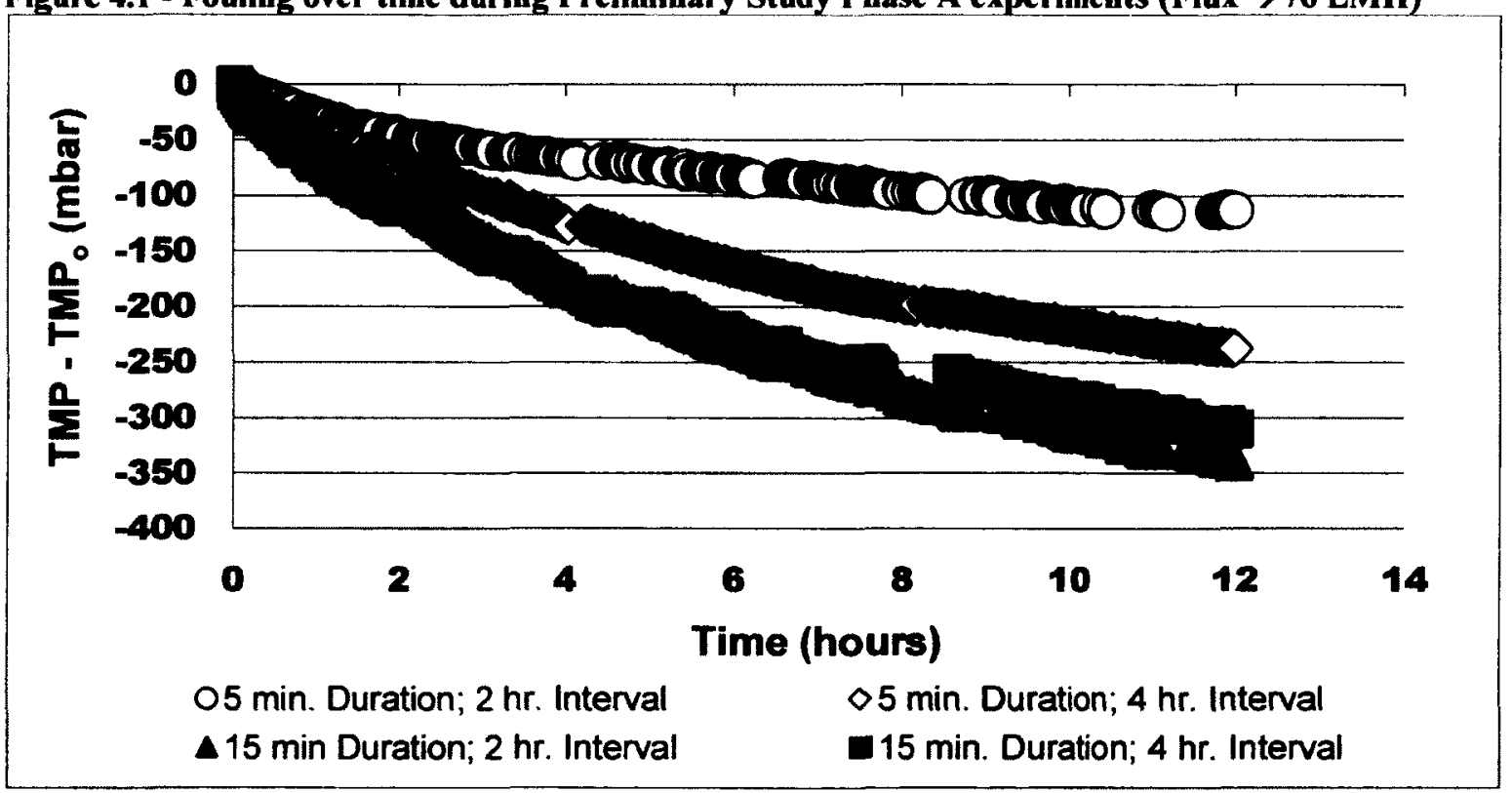

The $\triangle T M P$ for each run of the Preliminary Study Phase A experiments was measured by finding the difference between the minimum TMP measured in the first 60 seconds of each experiment and the minimum TMP measured during the final 300 
seconds of the experiment. The resulting $\triangle T M P$ for the Preliminary Study Phase A experiments can be seen in Table 4.1.

Table 4.1 - $\triangle$ TMP (mbar) observed for Preliminary Study Phase A experiments

\begin{tabular}{|c|c|c|c|c|}
\cline { 2 - 4 } \multicolumn{2}{c|}{} & \multicolumn{2}{c|}{ Interval } & \multicolumn{1}{c|}{$\begin{array}{c}\text { Row } \\
\text { Average }\end{array}$} \\
\cline { 2 - 5 } & 2 hours & 4 hours & -176 \\
\hline \multirow{2}{*}{ Duration } & $5 \mathrm{~min}$ & -114 & -239 & -326 \\
\cline { 2 - 5 } & $15 \mathrm{~min}$ & -340 & -311 & -251 \\
\hline \multicolumn{2}{|c|}{ Column Average } & -227 & -275 & - \\
\hline
\end{tabular}

On comparing the row and column averages with the overall average of -251 mbar, it can be seen that the relaxation duration had a greater effect on $\triangle T M P$ than the interval between relaxation periods. The difference in the averages from the overall mean is highlighted in the main effects calculated using Equations 3.1 and 3.2 (CHAPTER 3 Section 3.5.1) and shown in Table 4.2. From this table it can be seen that the main relaxation duration effects are larger than the main relaxation interval effects implying that duration has more of an effect on $\triangle T M P$. However these main effects were not additive indicating the presence of an interaction effect between relaxation duration and interval. The interaction effect was calculated using Equation 3.4 and included in Table 4.2. An interaction plot was also generated using $\triangle \mathrm{TMP}$ in Table 4.1 to identify the presence of the interaction and study the relationship between the interacting effects. 
Table 4.2 - Main and Interaction effects for Preliminary Study Phase A experiments

\begin{tabular}{|l|l|}
\hline \multicolumn{2}{|l|}{ Main duration effects } \\
\hline$\alpha_{1}$ & 74.8 \\
\hline$\alpha_{2}$ & -74.8 \\
\hline Main Interval effects & \\
\hline$\beta_{1}$ & 23.9 \\
\hline$\beta_{2}$ & -23.9 \\
\hline Interaction effects & \\
\hline$\alpha \beta_{11}$ & 38.4 \\
\hline$\alpha \beta_{12}$ & -38.4 \\
\hline$\alpha \beta_{21}$ & -38.4 \\
\hline$\alpha \beta_{22}$ & 38.4 \\
\hline
\end{tabular}

The lines in the interaction plot (Figure 4.2) lack parallelism and thus confirm the existence of an interaction between relaxation duration and the interval between relaxations. To test the importance of the interaction, simple data transformations such as the logarithmic, square, square root and reciprocal transformations were performed in attempts of making the existing interaction unimportant. However, the interaction was found to be non-transformable as the data transformations failed in making the interactions unimportant. Thus conclusions on the effect of relaxation duration and interval were made taking the presence of the interaction into account.

Figure 4.2 - Effect of duration vs. interval on $\triangle T M P$ in Preliminary Phase A experiments (76 LMH)

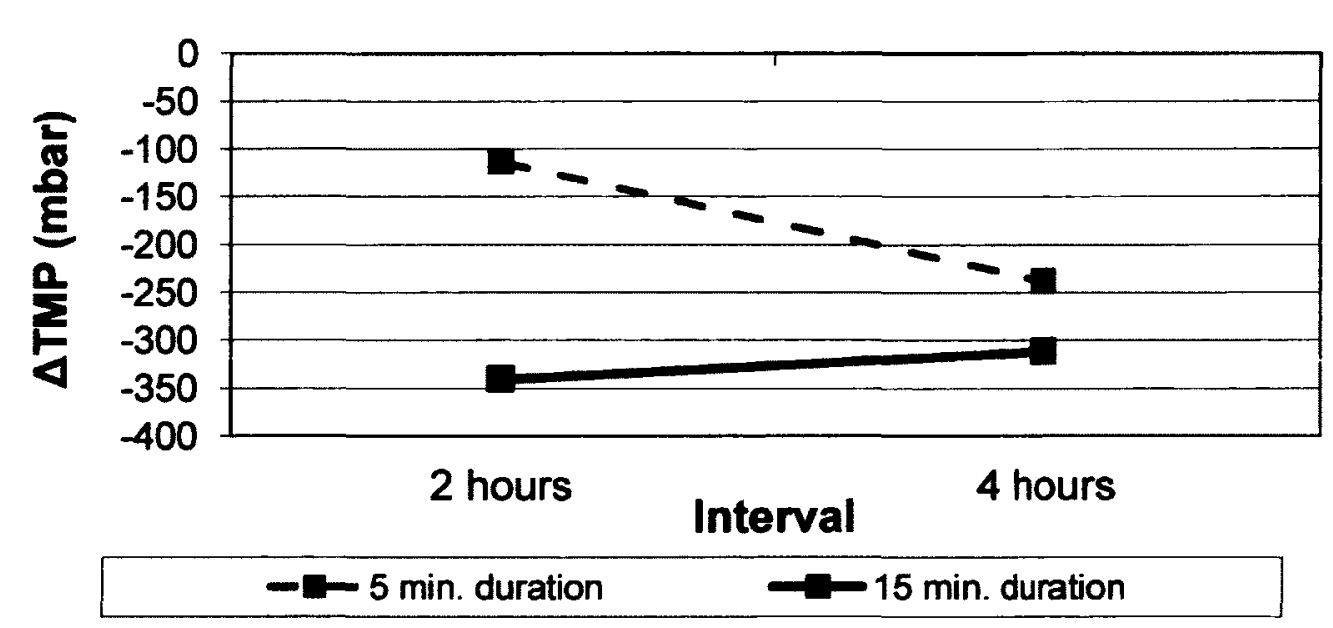


From Figure 4.1 and Figure 4.2 it can be seen that increasing the interval between relaxations when using the shorter relaxation duration $(5 \mathrm{~min}$.) resulted in an increase in fouling. This increase in TMP could be due to the higher accumulation of the cake layer during the longer interval ( $4 \mathrm{hrs}$.) compared to the shorter interval ( $2 \mathrm{hrs}$.) between relaxations. These results imply that frequent and short periods of relaxation are required to ensure less accumulation of fouling on the membrane surface and thus reduce fouling. At the longer relaxation duration (15 min.), an increase in the relaxation interval resulted in a slight decrease in fouling.

While it was expected that the use of longer relaxation durations would result in lower fouling due to the longer period of time for the cleaning action of air scouring on a relaxed membrane surface, the results from this preliminary phase study proved otherwise. This unexpected increase in fouling at the longer relaxation duration was attributed to the complete mixing of the solution in the reactor tank over the 15 minutes resulting in the absence of a foulant concentration gradient at the surface of the membrane. Instead, due to the homogenous nature of the mixed solution at the end of a 15 minute relaxation period, the membrane surface was exposed to a high concentration of foulants at the start of a forward filtration flow following a relaxation period thus resulting in an immediate buildup of the cake layer. Furthermore, the concentration of the homogenous mixture increased over time due to the employment of the dead-end flow operation thus compounding the effect of complete mixing in the reactor tank on fouling.

Based on these observations, the operating conditions used and taking into account the presence of an interaction effect, the duration of relaxation was found to have a greater effect on fouling reduction than the interval between relaxations. The results of 
Phase $\mathrm{A}$ indicated that shorter relaxation durations at shorter relaxation intervals were more beneficial for reduction of membrane fouling than longer duration, longer intervals relaxations modes.

\subsubsection{Phase A Water Quality Assessment}

Samples were taken from the feed, reactor and permeate (backwash) tanks at the beginning and end of each experimental run. Samples were analysed for $\mathrm{pH}$, turbidity and chemical oxygen demand COD. The results of these water quality tests can be seen in APPENDIX $\mathrm{J}$. The $\mathrm{pH}$ of the solution was found to remain fairly constant in each tank throughout each experimental run. In the case of turbidity and COD, as expected when using a dead-end flow operation, the accumulation of suspended solids and COD in the reactor tank increased over time. This increase in concentration was deemed to be responsible for the high fouling of the membrane unit over time thus resulting in loss of membrane productivity.

\subsection{Preliminary Phase B Results}

Preliminary Study Phase A experiments were run under high fouling conditions which included a high flux (76 LMH) and dead-end flow operation. However it was determined that the fouling was potentially too high for meaningful results therefore Phase B experiments assessed dead-end versus continuous flow operation modes. The filtration and backwash fluxes were also decreased to $38 \mathrm{LMH}$. 
The use of a continuous flow operation was thought to be a better alternative to the dead-end flow operation used in Phase A experiments specifically for minimizing the accumulation of foulants while maintaining a consistent feed water quality in the reactor tank over time. Two experimental sets were thus employed during Preliminary Phase B, one employing a dead-end flow operation while the other employed a continuous flow operation as described in CHAPTER 3.

In the case of the experimental set employing continuous flow operation, feed flow into the reactor tank was set such that an $80 \%$ recovery of permeate from the reactor was achieved. The operational parameters and relaxation mode regimes for both experimental sets employed in Preliminary Study Phase B can be seen in Chapter 3 Section 3.2.1.2. The following sections inform on the results and findings from Preliminary Study Phase B.

\subsection{1 $\triangle T M P$}

Fouling was measured in terms of TMP readings logged every 15 second during forward filtration flow and relaxation periods and at every second during backwash periods for all Phase B experiments. For each experimental run, the change in TMP ( $\triangle T M P)$ was calculated as the difference between the lowest peak detected within the first 15 minutes of the run and the lowest peak detected within the final 15 minutes of the experimental run. The 15 minute period at the start and end of each run was used to ensure a proper representation of the fluctuating TMP during calculations of $\triangle T M P$. For ease of analysis of $\triangle \mathrm{TMP}$, the results from the dead-end operation and continuous flow 
operation experiments were analyzed separately before being combined to analyse the effect of the two flow operations on fouling. The following sections show the benefit of including a relaxation mode in an operational regime as well as discuss the analysis of the $2^{2}$ factorial experiments results obtained for each flow operation and the analysis of a pseudo $2^{3}$ factorial design combining both $2^{2}$ factorial data sets to compare the $\Delta$ TMP results from each set of experiments.

\subsubsection{Relaxation vs. No Relaxation}

Each set of Preliminary Study Phase B was made up of a $2^{2}$ factorial study investigating the effect of relaxation duration and interval at the two flow operations on fouling as well as an additional experimental run to test the fouling impact of excluding relaxations from the treatment combinations used in the $2^{2}$ factorial studies. Figure 4.3 compares the change in TMP observed for experiments without relaxation and the change in TMP of the most fouling experiment with relaxation from each set. From this figure it can be seen that the exclusion of relaxation resulted in much higher fouling than experiments involving relaxation. Thus, the inclusion of relaxation in an operational regime with continuous air scouring is extremely beneficial for reducing membrane fouling. 
Figure 4.3 - The effect of relaxation exclusion on fouling under continuous flow (CF) and dead-end flow (DE) operations (Flux $\rightarrow 38$ LMH)

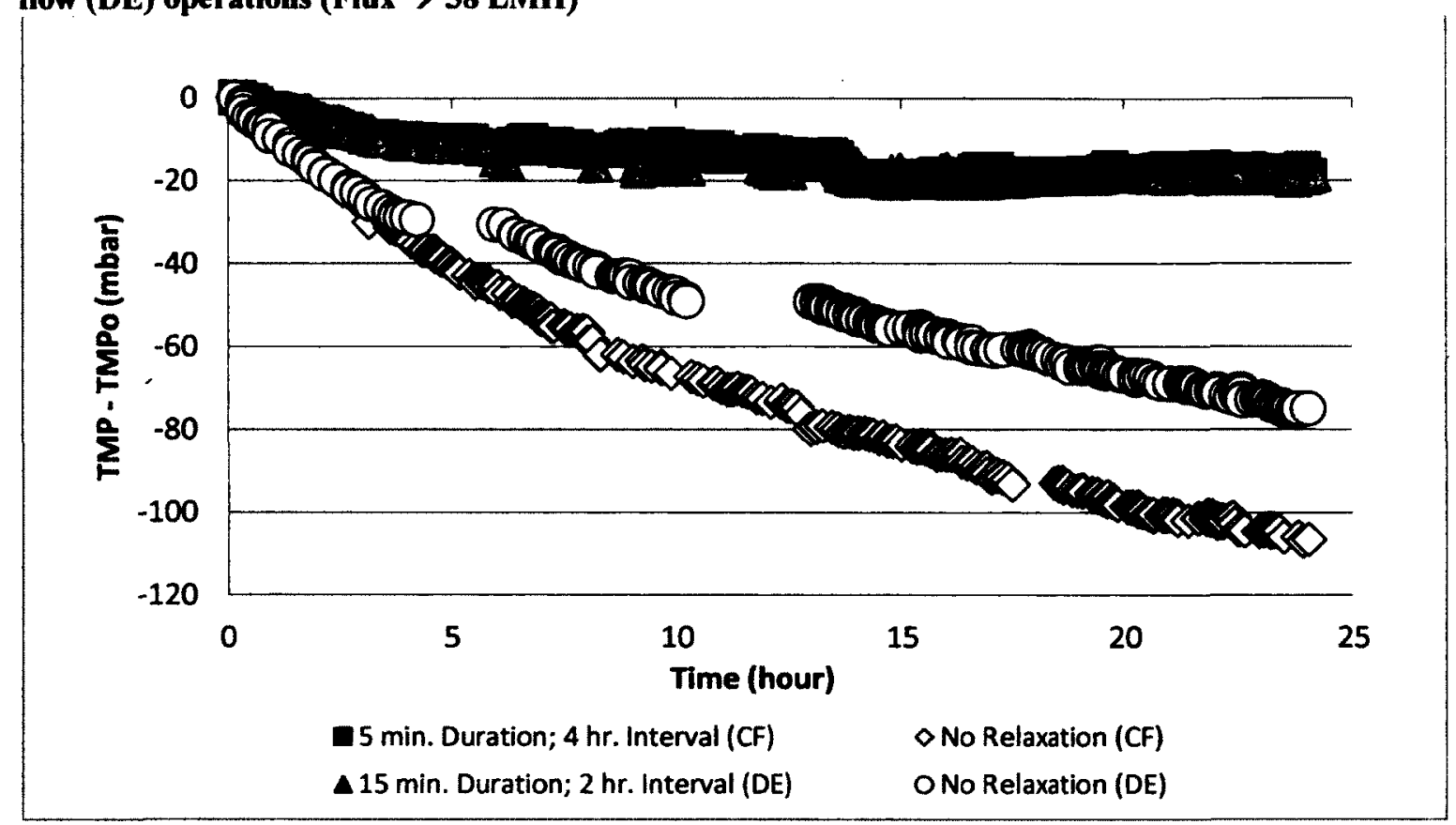

\subsubsection{Dead-end Flow Operations}

The change in TMP over the $2^{2}$ factorial experiments employing a dead-end flow operation can be seen in Figure 4.4. Similar to the findings in the Preliminary Study Phase A results, the use of longer relaxation duration resulted in higher fouling than the use of the shorter relaxation duration. As in the Phase A results, the higher fouling observed when using longer relaxation durations could be due to the homogeneity of the solution in the reactor tank caused by complete mixing of the solution by the rising air bubbles. 


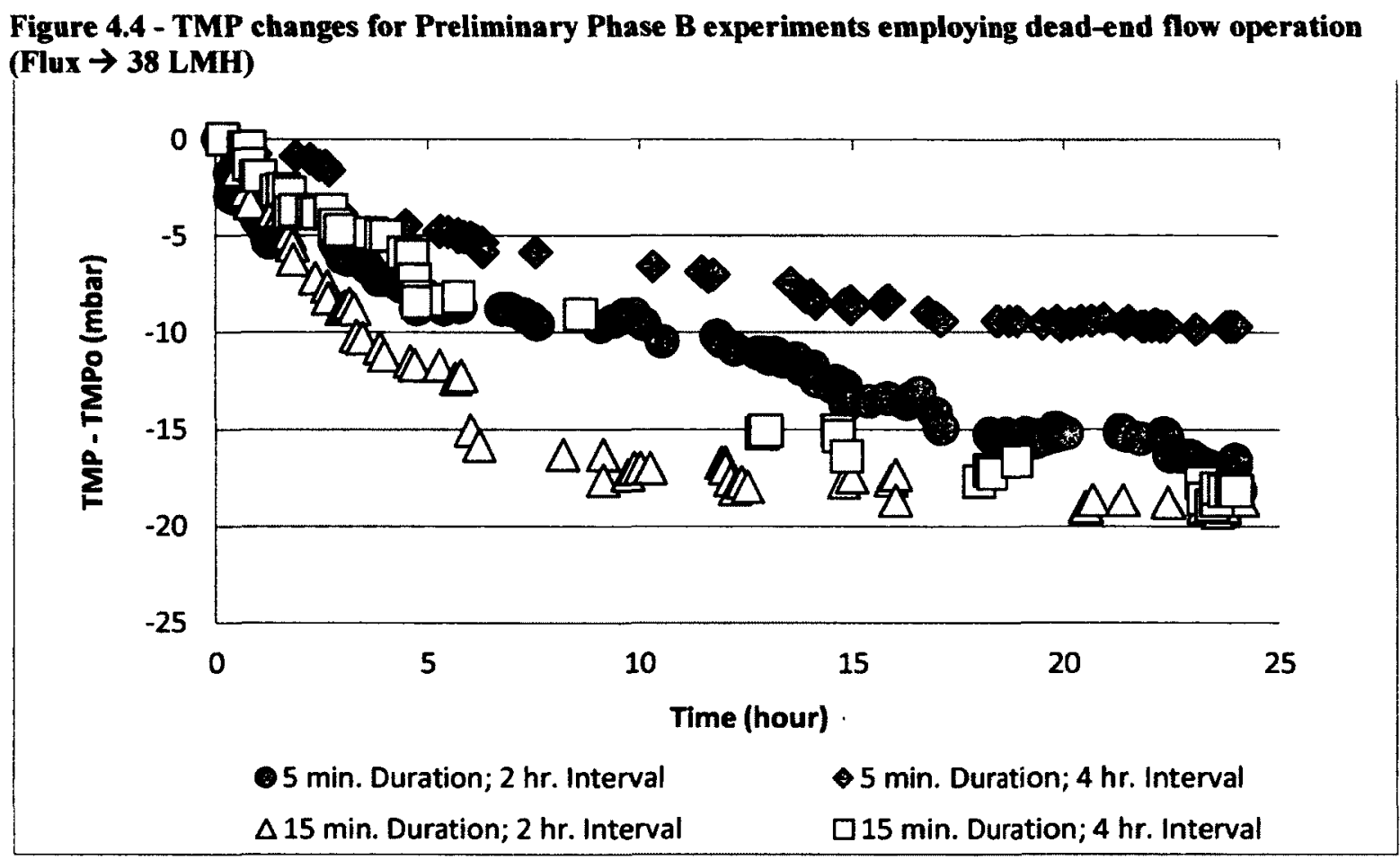

In comparison to the Preliminary Phase $\mathrm{A}$ results where shorter relaxation and shorter relaxation interval resulted in the lowest TMP change; here the shorter relaxation with longer relaxation interval resulted in the lowest TMP change with the system. This change in fouling behaviour could be attributed to the effect of the different filtration fluxes used during the two phases on the compactness of the cake layer formed on the membrane surface. While the longer relaxation interval may result in a greater accumulation of foulants between relaxation periods than the shorter interval, the cake layer formed would be less compact in Phase B dead-end flow experiments than Phase A dead-end flow experiments due to the lower TMP needed for the required permeate flow (i.e. a final TMP ranging between -240 to -310 mbar for Phase A experiments versus -10 to -18 mbar for Phase $B$ experiments.). Thus air scouring during relaxation would be potentially more effective in removing foulants off the membrane surface in Phase B 
experiments. The larger accumulation of foulant on the surface might also serve as an advantage during air scouring by obstructing the air bubble pathway thus increasing friction between the air bubbles and fouling surface and promoting the displacement of larger foulant clusters by the air bubbles.

As in the case of the Phase A experiments, a two way ANOVA model was developed for Phase B dead-end flow experiments as portrayed in Table 4.3. On comparing the change in TMP of the four treatment combinations used in the 24 hour Phase B dead-end experiments it can be seen that a change in relaxation duration at 2 hour intervals as well as a change in interval when using 15 minute relaxation periods did not affect the fouling of the membrane unit.

Table 4.3 - $\triangle T$ TMP (mbar) observed for Phase B dead-end flow experiments

\begin{tabular}{|c|c|c|c|c|}
\cline { 2 - 4 } \multicolumn{2}{c|}{} & \multicolumn{2}{c|}{ Interval } & \multicolumn{1}{c|}{$\begin{array}{c}\text { Row } \\
\text { Average }\end{array}$} \\
\cline { 2 - 4 } & 2 hours & 4 hours & \\
\hline \multirow{2}{*}{ Duration } & $5 \mathrm{~min}$ & -17.2 & -9.72 & -13.4 \\
\cline { 2 - 4 } & $15 \mathrm{~min}$ & -17.9 & -18.2 & -18.1 \\
\hline \multicolumn{2}{|c|}{ Column Average } & -17.5 & -14.0 & -15.8 \\
\hline
\end{tabular}

On comparing the main effects of relaxation duration and interval shown in Table 4.4 it can be seen that both duration and interval have an effect on the change in TMP, however an interaction effect is also present. The interaction plot shown in Figure 4.5 shows the importance of the interaction between relaxation duration and the interval between relaxations when using a dead-end flow operation. Data transformations used in 
the analysis of the Preliminary Phase A experiments were also used to test the importance of the existing interaction in Phase $B$ and showed that the interaction was non transformable. Thus based on the presence of the important interaction, it was concluded that for treatments using dead-end flow and a filtration flux meeting the membrane requirements, fouling can be reduced by using shorter durations and longer intervals between relaxations.

Table 4.4 - Main and interaction effects for Phase B dead-end flow experiments.

\begin{tabular}{|l|l|}
\hline \multicolumn{2}{|l|}{ Main duration effects } \\
\hline $\boldsymbol{\alpha}_{\mathbf{1}}$ & 2.31 \\
\hline $\boldsymbol{\alpha}_{\mathbf{2}}$ & -2.31 \\
\hline Main Interval effects \\
\hline $\boldsymbol{\beta}_{\mathbf{1}}$ & -1.79 \\
\hline $\boldsymbol{\beta}_{\mathbf{2}}$ & 1.79 \\
\hline Interaction effects \\
\hline $\mathbf{\alpha} \boldsymbol{\beta}_{\mathbf{1 1}}$ & -1.93 \\
\hline $\mathbf{\alpha} \boldsymbol{\beta}_{\mathbf{1 2}}$ & 1.93 \\
\hline $\mathbf{\alpha} \boldsymbol{\beta}_{\mathbf{2 1}}$ & 1.93 \\
\hline $\mathbf{\alpha} \boldsymbol{\beta}_{\mathbf{2 2}}$ & -1.93 \\
\hline
\end{tabular}

Figure 4.5 - Effect of duration vs. interval on $\triangle T M P$ in Phase B dead-end flow experiments (Flux $\rightarrow$ 38 LMH)

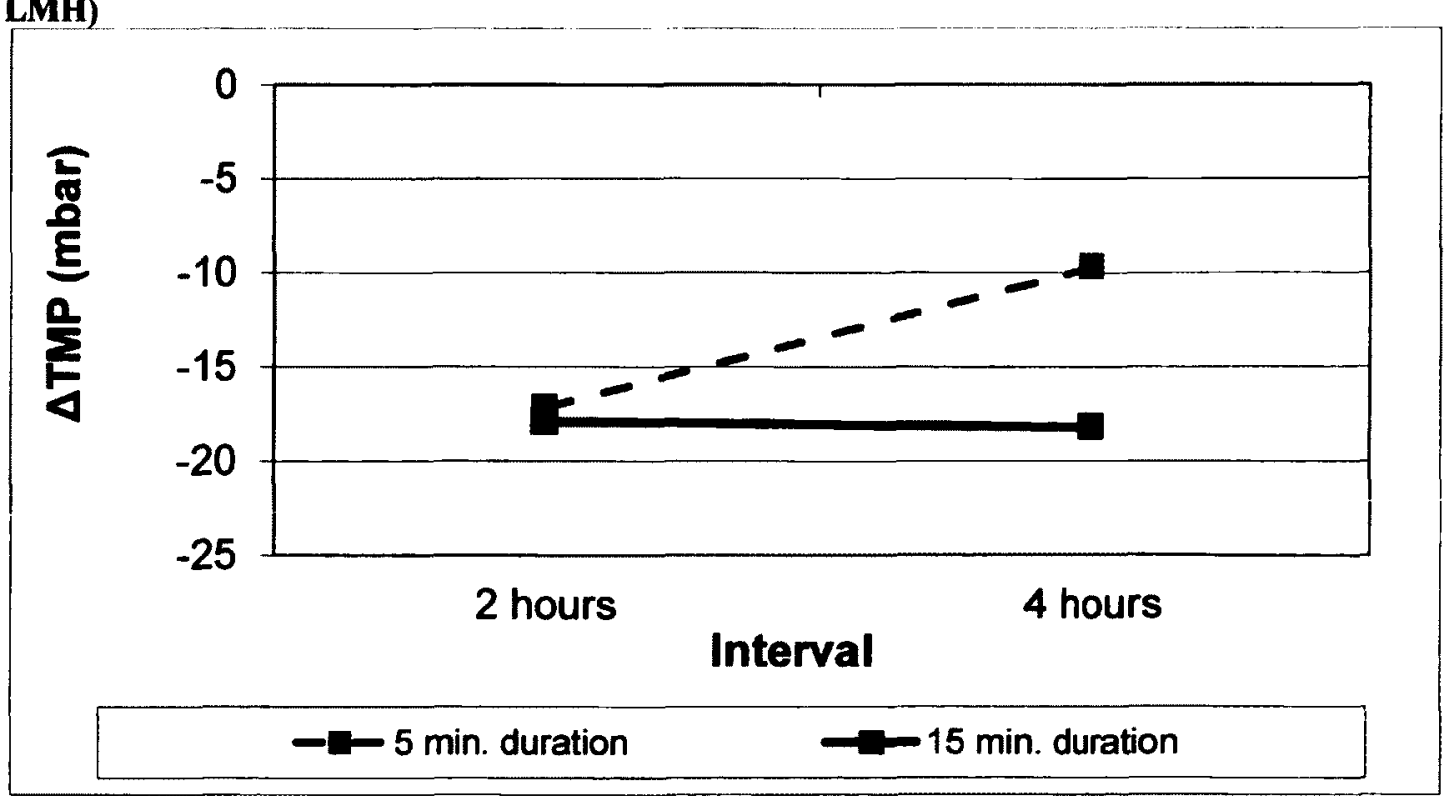




\subsubsection{Continuous Flow Operations}

The change in TMP observed during the Preliminary Phase B experiments employing a continuous flow operation with $80 \%$ recovery can be seen in Figure 4.6 .

Figure 4.6 - TMP changes for Phase B experiments employing a continuous now (80\% recovery) operation (Flux $\rightarrow 38$ LMH)

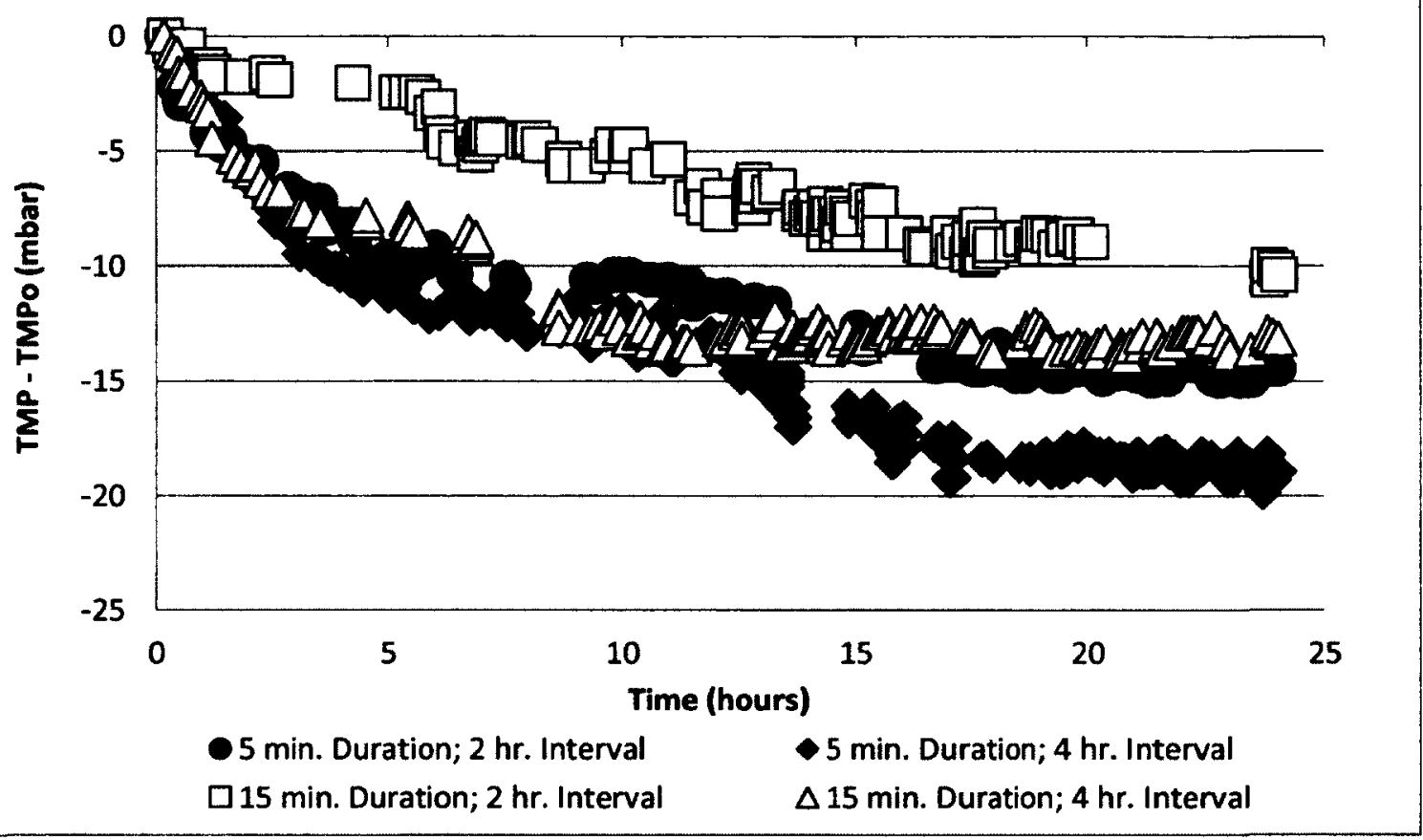

From the figure it can be seen that contrary to the results from experiments employing a dead-end flow operation, the use of longer relaxation durations results in less fouling than the use of shorter relaxation durations. While mixing takes place during the 15 minute relaxation period, overflow of retentate also takes place. Consequently, the concentration in the reactor tank decreases over time during the 15 minute relaxation period and any immediate and extreme fouling at the start of filtration following a relaxation period may be minimized. 
The change in TMP over the 24 hour long Phase B continuous flow experiments is shown in Table 4.5. From the table it can be seen that the use of short duration period at longer intervals and a continuous flow operation with $80 \%$ recovery resulted in the highest fouling of the four treatment combinations. The determined main duration effects in Table 4.6 show that duration had a greater effect on fouling than interval. While an interaction effect was also determined, a log transformation of the response data showed that the interaction was unimportant and thus was neglected when making conclusions on the effect of relaxation duration and interval when using a continuous flow operation.

Table 4.5 - $\triangle T M P$ (mbar) observed for Phase B continuous flow experiments

\begin{tabular}{|c|c|c|c|c|}
\cline { 2 - 5 } \multicolumn{2}{c|}{} & \multicolumn{2}{c|}{ Interval } & \multicolumn{1}{c|}{$\begin{array}{c}\text { Row } \\
\text { Average }\end{array}$} \\
\cline { 2 - 5 } \multicolumn{2}{c|}{ Duration } & 2 hours & 4 hours & \\
\cline { 2 - 5 } & $5 \mathrm{~min}$ & -14.5 & -19.3 & -16.9 \\
\hline & $15 \mathrm{~min}$ & -10.6 & -13.3 & -12.0 \\
\hline \multicolumn{2}{|c|}{ Column Average } & -12.5 & -16.3 & -14.4 \\
\hline
\end{tabular}

Table 4.6 - Main and interaction effects for Phase B continuous flow experiments.

\begin{tabular}{|l|c|}
\hline \multicolumn{2}{|l|}{ Main duration effects } \\
\hline $\boldsymbol{\alpha}_{1}$ & -2.54 \\
\hline $\boldsymbol{\alpha}_{2}$ & 2.54 \\
\hline \multicolumn{2}{|l|}{ Main Interval effects } \\
\hline $\boldsymbol{\beta}_{1}$ & 1.88 \\
\hline $\boldsymbol{\beta}_{2}$ & -1.88 \\
\hline Interaction effects \\
\hline $\boldsymbol{\alpha} \boldsymbol{\beta}_{11}$ & 0.53 \\
\hline $\boldsymbol{\alpha} \boldsymbol{\beta}_{12}$ & -0.53 \\
\hline $\boldsymbol{\alpha} \boldsymbol{\beta}_{21}$ & -0.53 \\
\hline $\boldsymbol{\alpha} \boldsymbol{\beta}_{22}$ & 0.53 \\
\hline
\end{tabular}


Figure 4.7 shows that for both relaxation durations, a shorter relaxation interval resulted in less fouling, with the use of the longer 15 minute duration resulting in the least fouling. Since interaction effects were deemed unimportant it was said that fouling can be reduced by using shorter intervals between relaxations when using a continuous flow operation.

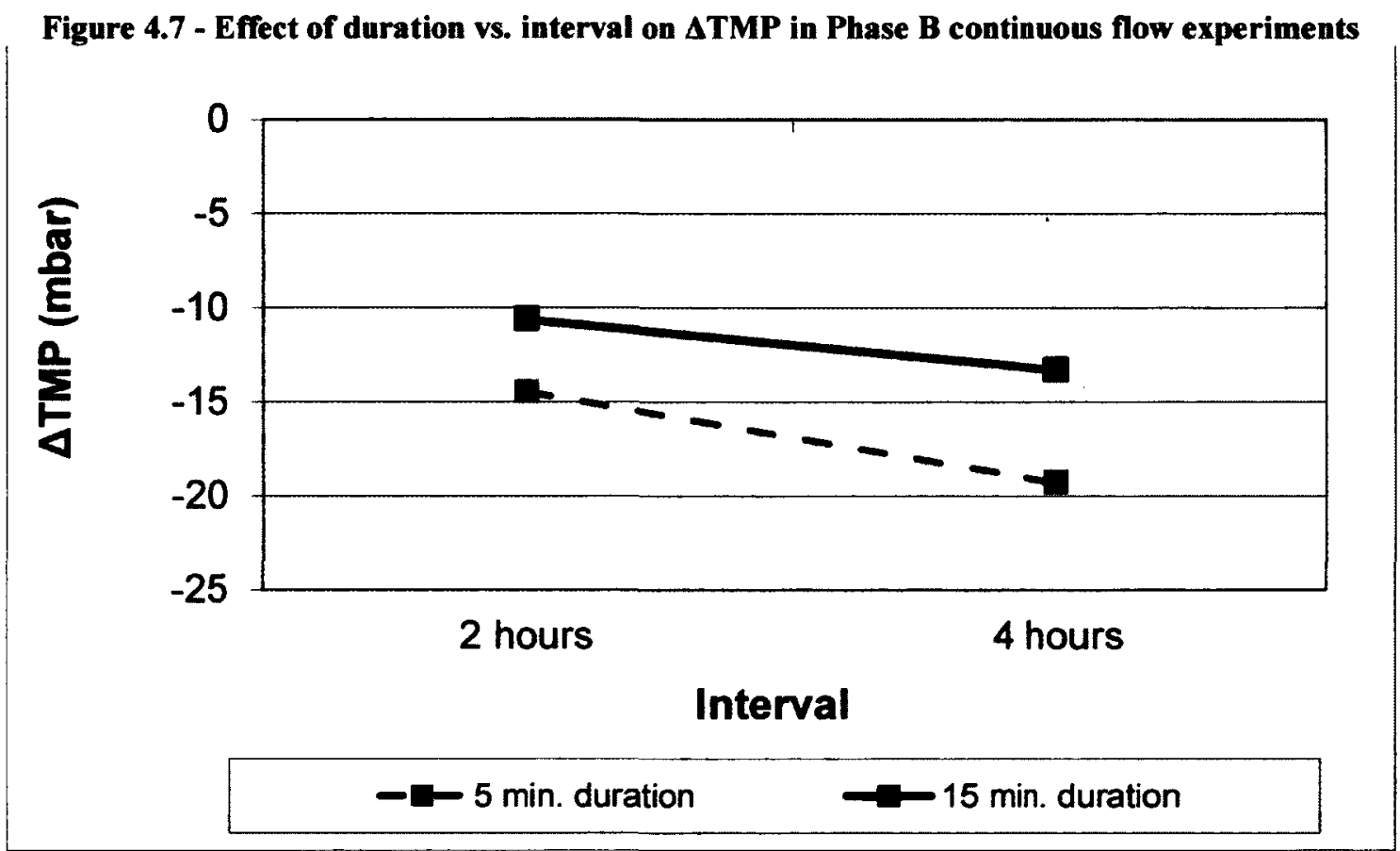

\subsubsection{Dead-end Flow vs. Continuous Flow}

While both Preliminary Study Phases were performed as two separate $2^{2}$ factorial studies, results from both phases were combined together to form a pseudo $2^{3}$ factorial study with relaxation duration and interval as well as flow operation (dead-end flow and continuous flow) as the three factors. This combination was performed for the sole 
purpose of comparing the effect of dead-end flow and continuous flow operation on membrane fouling.

Table 4.7 - Effects for the comparison of Continuous flow vs. Dead-end flow operations

\begin{tabular}{|c|c|}
\hline$a_{2}$ & 0.607 \\
\hline$\beta_{2}$ & 0.021 \\
\hline$\gamma_{2}$ & 0.013 \\
\hline$\alpha \boldsymbol{\beta}_{22}$ & 2.44 \\
\hline$a \gamma_{22}$ & -1.89 \\
\hline$\beta \gamma_{22}$ & -0.75 \\
\hline$\alpha \beta \gamma_{222}$ & 1.29 \\
\hline
\end{tabular}

Main and interaction effects shown in Table 4.7 were calculated using a 3 factor ANOVA model developed by combining both Preliminary Study Phase B sets of experiments. From Table 4.7 it can be seen that the unusual occurrence of interaction effects being larger than main factor effects is present. Data transformations were performed which showed that all three interactions were important. Interaction plots representing the three interactions were also generated to analyse the importance of the interactions and can be seen in Figure $4.8 \mathrm{a}, \mathrm{b}$ and $\mathrm{c}$. From the interaction plots it can be seen that in all three cases, the use of continuous flow operations had an opposite effect on fouling than the use of the dead-end flow. 
Figure 4.8 - Interactions between a) Flow operation and relaxation duration (5 and $15 \mathrm{~min}$.), b) Flow operation and relaxation interval ( 2 and $4 \mathrm{hrs}$.) and c) Relaxation duration (5 and $15 \mathrm{~min}$.) and interval ( 2 and $4 \mathrm{hrs}$.) from Preliminary Phase B experiments.

a) Flow Operation vs. Relaxation Duration

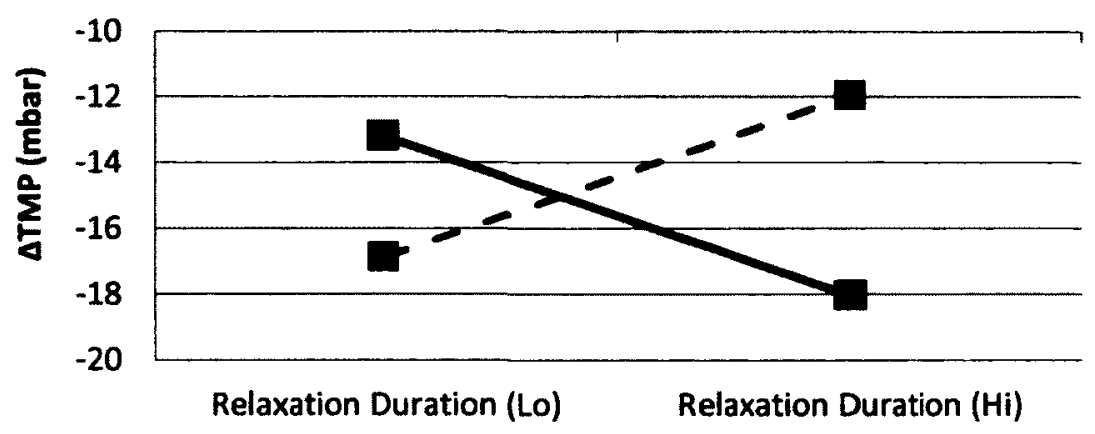

$\longrightarrow$ Dead End Flow Op. $\quad$ - Continuous (80\% recovery) Flow Op.

b) Flow Operation vs. Relaxation Interval

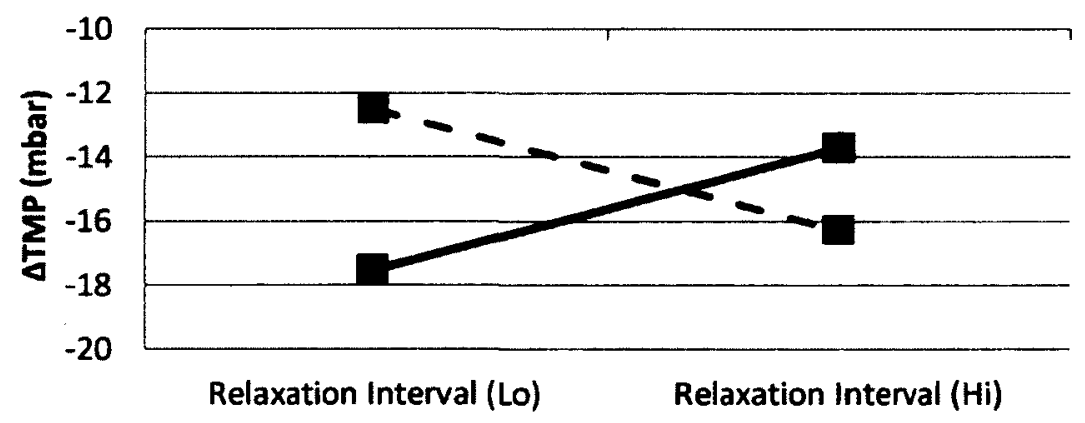

$\rightarrow$ Dead End Flow Op. $\quad-$ Continuous (80\% recovery) Flow Op.

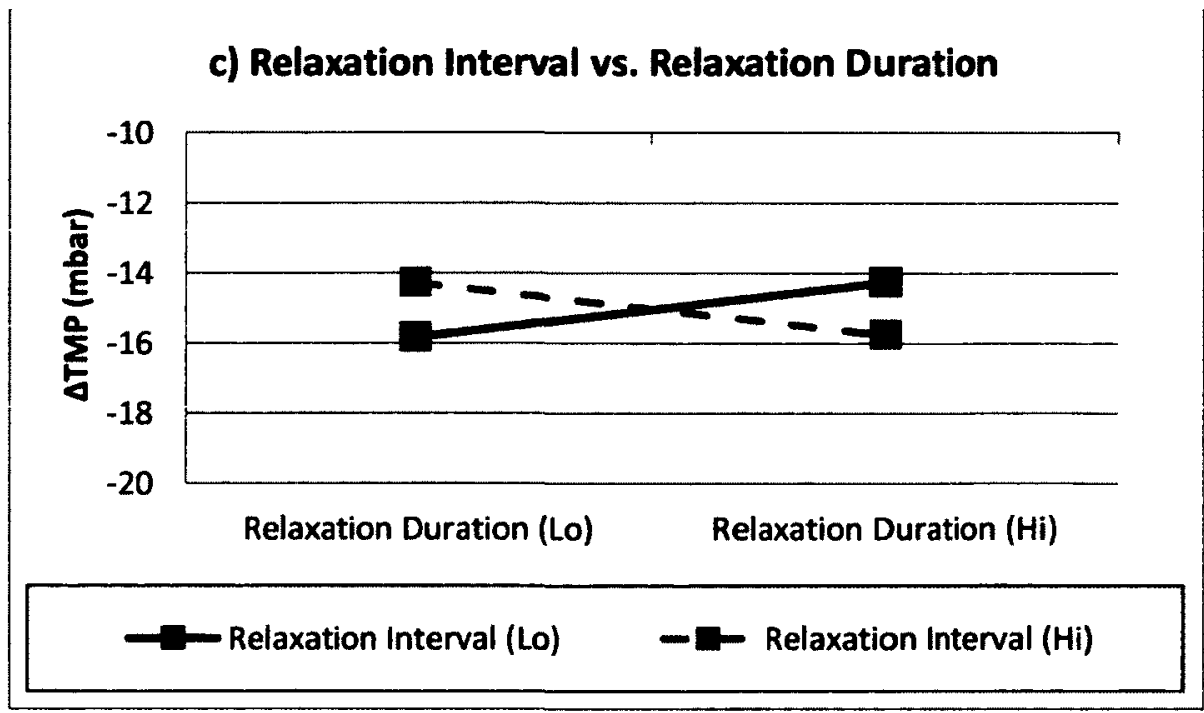


Figure 4.9 compares the change in TMP of the 8 treatment combinations performed in Preliminary Study Phase B and shows that experiments employing continuous flow operations experienced less fouling than those employing dead-end flow operations except for the combinations using a 5 min duration period at 4 hour intervals. The difference in TMP observed between the two flow operations explains the presence of such strong interaction effects when comparing the effect of flow operation on fouling. In most cases less fouling was observed when employing the continuous flow operation. However, to confirm fouling analysis by TMP, an analysis of the water quality results was also conducted.

Figure 4.9 - Comparison of TMP changes in preliminary phase B experiments (Flux $\rightarrow 38$ LMH)

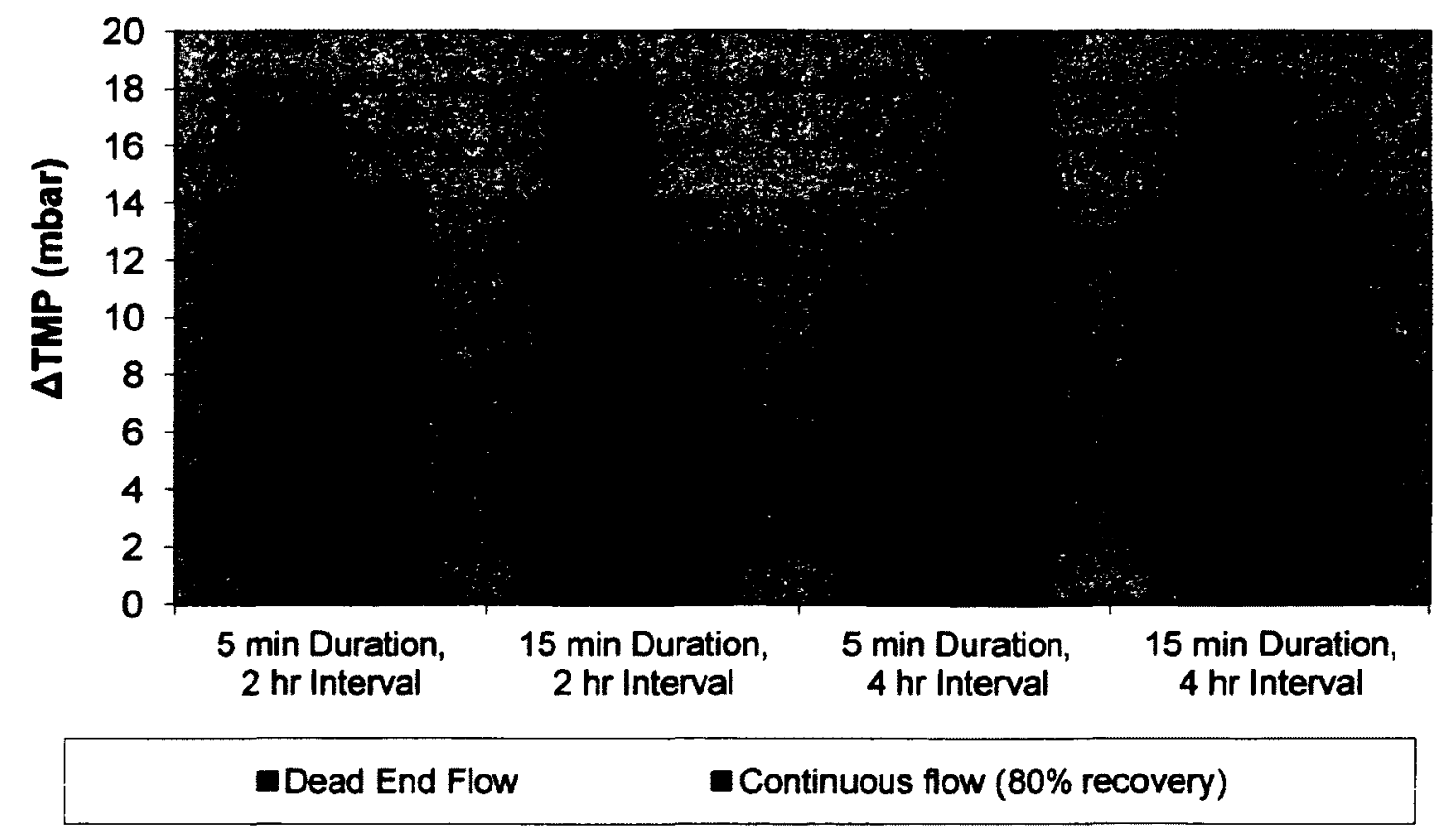




\subsubsection{Water Quality Assessment}

Samples of the solution in the reactor tank, feed tank as well as permeate were taken at the start, middle $(t=12$ hrs.) and end $(t=24$ hrs.) of each experiment. The $\mathrm{pH}$, turbidity and COD levels of these samples were measured to identify changes in water quality parameters over the course of each experiment and can be seen in APPENDIX K. Temperature measurements of the solution in the reactor tank were also taken at the start and end of each experiment and can be seen in Table 4.8 along with the turbidity and COD measured in the reactor tank.

On average, 6 and 5 fold increases in turbidity and COD respectively were experienced for experiments using a dead-end flow operation and involving relaxation. In comparison, only a 2 fold increase in turbidity as well as COD was experienced for experiments employing a continuous flow operation with $80 \%$ recovery as well as relaxation. On further observation it can be seen that in the case of the continuous flow experiments, the 2 fold increases were experienced after 12 hours of the experimental runs after which no more increase in the turbidity and COD took place. Thus, a steady state was reached when using continuous flow operations.

On comparing the temperature changes between the two flow operations it can be seen that the temperature remains constant in the reactor tank for most experiments employing continuous flow operation while the temperature drops when using a dead-end flow operation. Thus steady state conditions are achieved for COD, turbidity as well as temperature making the continuous flow operation ideal for the longer experimental runs. 
Table 4.8 - Comparison of Turbidity, COD and Temperature in the reactor tank for Phase B experiments

\begin{tabular}{|c|c|c|c|}
\hline Sample & $\begin{array}{l}\text { Turbidity } \\
\text { (NTU) }\end{array}$ & COD (mg/L) & Tomp. $\left({ }^{\circ} \mathrm{C}\right)$ \\
\hline \multicolumn{4}{|c|}{ Dead-end flow - Base (no relaxation) } \\
\hline Reactor (Initial) & 26.90 & 13.5 & 23.5 \\
\hline Reactor (12 hours) & 62.19 & 32.0 & - \\
\hline Reactor ( 24 hours) & 44.94 & 13.0 & 20.0 \\
\hline \multicolumn{4}{|c|}{ Dead-end flow - 2 hr interval; 5 min Duration } \\
\hline Reactor (Initial) & 29.14 & 15.0 & 24.0 \\
\hline Reactor ( 12 hours) & 117.6 & 55.0 & - \\
\hline Reactor ( 24 hours) & 169.5 & 62.5 & 22.0 \\
\hline \multicolumn{4}{|c|}{ Dead-end flow - $2 \mathrm{hr}$ interval; $15 \mathrm{~min}$ Duration } \\
\hline Reactor (Initial) & 26.84 & 13.0 & 24.0 \\
\hline Reactor (12 hours) & 113.7 & 42.0 & - \\
\hline Reactor (24 hours) & 155.4 & 53.0 & 23.0 \\
\hline \multicolumn{4}{|c|}{ Dead-end flow - 4 hr Interval; 5 min Duration } \\
\hline Reactor (Initial) & 29.66 & 9.50 & 25.0 \\
\hline Reactor ( 12 hours) & 101.6 & 44.0 & - \\
\hline Reactor ( 24 hours) & 125.2 & 46.5 & 22.0 \\
\hline \multicolumn{4}{|c|}{ Dead-end flow - $4 \mathrm{hr}$ Interval; 15 min Duration } \\
\hline Reactor (Initial) & 30.17 & 9.50 & 24.0 \\
\hline Reactor ( 12 hours) & 101.6 & 56.0 & - \\
\hline Reactor (24 hours) & 188.2 & 68.5 & 21.0 \\
\hline \multicolumn{4}{|c|}{ Continuous flow - Base (no relaxation) } \\
\hline Reactor (Initial) & 29.46 & 17.0 & 24.50 \\
\hline Reactor (12 hours) & 46.07 & 19.5 & - \\
\hline Reactor (24 hours) & 37.01 & 19.0 & 25.00 \\
\hline \multicolumn{4}{|c|}{ Continuous flow - 2 hr Interval; 5 min Duration } \\
\hline Reactor (Initial) & 30.23 & 13.5 & 24.5 \\
\hline Reactor ( 12 hours) & 82.92 & 33.5 & - \\
\hline Reactor (24 hours) & 65.39 & 29.5 & 24.5 \\
\hline \multicolumn{4}{|c|}{ Continuous flow - $2 \mathrm{hr}$ interval; $15 \mathrm{~min}$ Duration } \\
\hline Reactor (Initial) & 30.92 & 16.0 & 24 \\
\hline Reactor ( 12 hours) & 61.72 & 25.5 & - \\
\hline Reactor ( 24 hours) & 62.87 & 32.5 & 24.5 \\
\hline \multicolumn{4}{|c|}{ Continuous flow - $4 \mathrm{hr}$ Interval; $5 \mathrm{~min}$ Duration } \\
\hline Reactor (Initial) & 30.81 & 20.0 & 24.5 \\
\hline Reactor ( 12 hours) & 71.04 & 31.5 & - \\
\hline Reactor ( 24 hours) & 68.21 & 35.5 & 24.5 \\
\hline \multicolumn{4}{|c|}{ Continuous flow - $4 \mathrm{hr}$ Interval; $15 \mathrm{~min}$ Duration } \\
\hline Reactor (Initial) & 26.47 & 14.0 & 25.0 \\
\hline Reactor ( 12 hours) & 75.66 & 36.5 & - \\
\hline Reactor (24 hours) & 70.60 & 27.5 & 25.0 \\
\hline
\end{tabular}




\subsection{Preliminary Study Phase Conclusions}

The Preliminary Study Phase was performed to identify appropriate operational parameters to be used in the fouling study performed in the Main Phase Experiments. The study phase itself was carried out in two sub phases; Preliminary Study Phase A and Preliminary Study Phase B.

In Preliminary Phase A, the membrane unit was subjected to high fouling conditions during which the effect of relaxation parameters in conjunction with air scouring and backwashing on fouling reduction was analysed. Relaxation duration ( 5 and 15 minutes) was found to have a greater effect on fouling reduction than relaxation interval ( 2 and 4 hours). Shorter relaxation duration at a shorter time interval was beneficial for reducing fouling in this phase. As expected when using a dead-end flow operation, organic matter and particulate matter was found to accumulate in the reactor tank over time. The increase in concentration of foulants in the reactor tank as well as high filtration flow rate used in Phase A experiments was deemed responsible for the high level of fouling observed.

In Preliminary Phase B, the membrane unit was subject to more operationally suitable filtration conditions. This phase investigated the effect of relaxation duration ( 5 and 15 minutes) and interval ( 2 and 4 hours) on fouling while employing two flow operations (dead-end flow and continuous flow). The effect of the flow operation on fouling as well as the effect of the exclusion of relaxation from an operational regime was also examined. The use of relaxation in an operational regime along with air scouring and backwashing was found to be extremely beneficial for the reduction of fouling. 
In Phase B dead-end flow experiments, relaxation duration was found to have a greater effect on fouling than relaxation interval with the longer duration resulting in higher fouling. Shorter relaxation duration ( $5 \mathrm{~min}$.) at a longer interval (4 hrs.) minimized fouling. Phase B continuous flow experiment results showed that shorter interval helped reduce fouling regardless of relaxation duration. Longer relaxation duration (15 min.) with a short interval ( 2 hrs.) helped achieve the least amount of fouling.

On comparing water quality data from Phase B experiments, steady state was achieved in the reactor tank over time when using a continuous flow operation. This along with the mostly lower fouling achieved during continuous flow experiments supported the use of continuous flow operations for Main Phase Experiments in order to assess a longer term impact of physical operational parameters on fouling (i.e. backwashing, air scouring, and relaxation). 


\section{CHAPTER 5 - Main Phase Experiments Results}

The main objective of the research project was to investigate the effect of physical cleaning operations specifically backwashing, relaxation and air scouring on the fouling reduction of a bench scale hollow fiber ultrafiltration membrane system. A $2^{4}$ factorial design of experiments was thus developed for a fouling study investigating the effect of the air flow rate, backwash duration and relaxation duration as well as the filtration flux on fouling. The Main Phase Experiments incorporated key findings from the Preliminary Study Phase experiments specifically the use of a continuous flow operation and the decision to use longer relaxation durations. Details on the factorial design as well as operational and water quality parameters used in the Main Phase Experiments can be seen in Chapter 3.

A 4-way ANOVA model along with interaction plots, general linear modelling and stepwise regression were used to identify important interactions between operational parameters and their corresponding effect on membrane fouling. The magnitudes of these parameters were used to identify important effects and interactions influencing membrane fouling. The model also allowed for the generation of two and three way interaction plots for the better understanding of the relationships between the various operational parameters and their effect on fouling. 
General linear modelling was used to confirm the main and interaction effect parameters estimated using the ANOVA model as well as provide another means of identifying important effects and interactions. These effects and interactions were identified by excluding individual effects and interactions from the original general linear model (GLM) for a $2^{4}$ factorial design of experiments and comparing the Sum of Squares of Residual error of the reduced model with that of the original GLM. Stepwise regression was eventually used to provide further statistical identification of important effects and interactions and provide a reduced GLM thought to best represent the data from the $2^{4}$ factorial design of experiments.

A fractional $2^{3}$ factorial design of experiments was also performed under this phase to study the exclusion effect of the cleaning operations on fouling. This factorial design of experiments was based on the original $2^{4}$ factorial experiment that resulted in the most fouling (Experiment 2).

\subsection{General Observations}

Error! Reference source not found. shows the fouling measured as TMP (mbar) at the end of the 5 day long $2^{4}$ factorial design experiments. Not surprisingly, the most (50.9 mbar) and least (-0.896 mbar) fouling was observed when physical cleaning operation parameters were at their lowest and highest levels respectively. In the case of the most fouling, the low levels of physical cleaning operational parameters were accompanied by a high filtration flux while high levels of cleaning operational parameters were accompanied by a low filtration flux for the least fouling experiment. 
The ranges of fouling caused by the high and low filtration flux were found to range from -8.96 to -50.9 mbar and -0.896 to -11.4 mbar respectively showing a high negative effect of filtration flux on fouling. However, due to the possible existence of interaction effects between filtration flux and the three cleaning operational parameters, the difference in fouling at the two flux levels cannot be attributed to filtration flux alone. The estimation of effects and interactions is thus required for the better understanding of the effect of the four operational parameters on fouling.

Table 5.1 - TMP change and permeate volume produced at the end of 5 day long Main Phase Experiments.

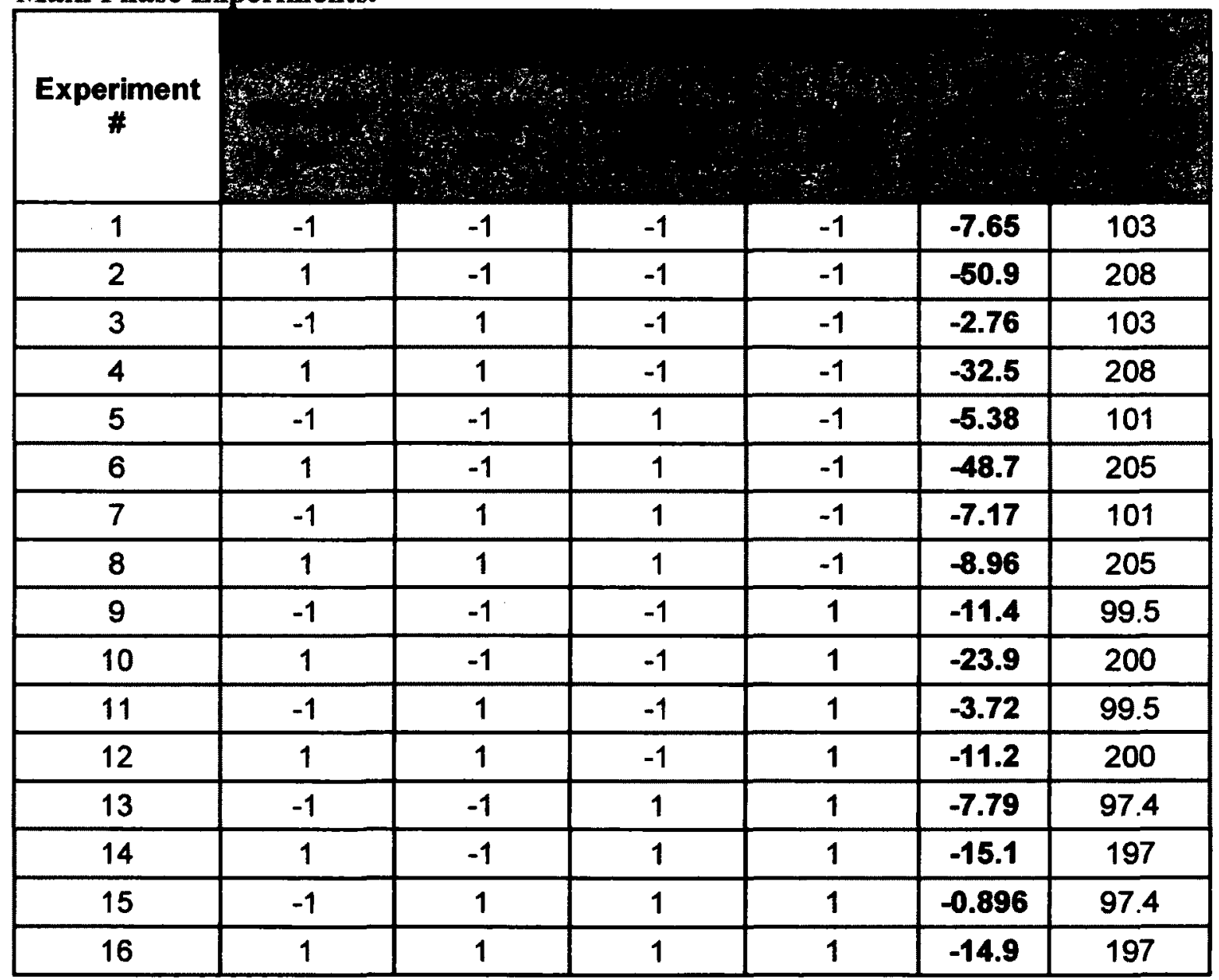

Note: 1) -1 and +1 denotes the low and high level of each parameter respectively.

2) The more negative the TMP value the greater the membrane fouling. 
Figure 5.1 visually compared the state of the membrane surface at the end of the most fouling (Experiment 2) and least fouling (Experiment 15) $2^{4}$ factorial design experiments. In Figure 5.1c, while the membrane surface was stained, no visible surface accumulation of material was evident following the least fouling experiment. This lack of cake formation on the membrane surface shows that the high air flow rate (15LPM) with long backwash duration ( $20 \mathrm{sec}$.) and long relaxation duration (15 min) was effective in helping remove foulants from the membrane surface at the low filtration flux. In the case of the high fouling experiment, surface fouling in Figure $5.1 \mathrm{~b}$ was fairly noticeable showing that the low air flow rate with short backwash and relaxation durations was not efficient at completely removing foulants off the membrane surface. This inability to remove the surface foulants using low levels of cleaning operation parameters could be due to the relatively high compactness of the foulant layer on the surface when using a high filtration flux compared to a low filtration flux.

Figure 5.1- State of membrane surface of a) a clean membrane, b) after Experiment 2 (most fouling) and c) after Experiment 15 (least fouling)

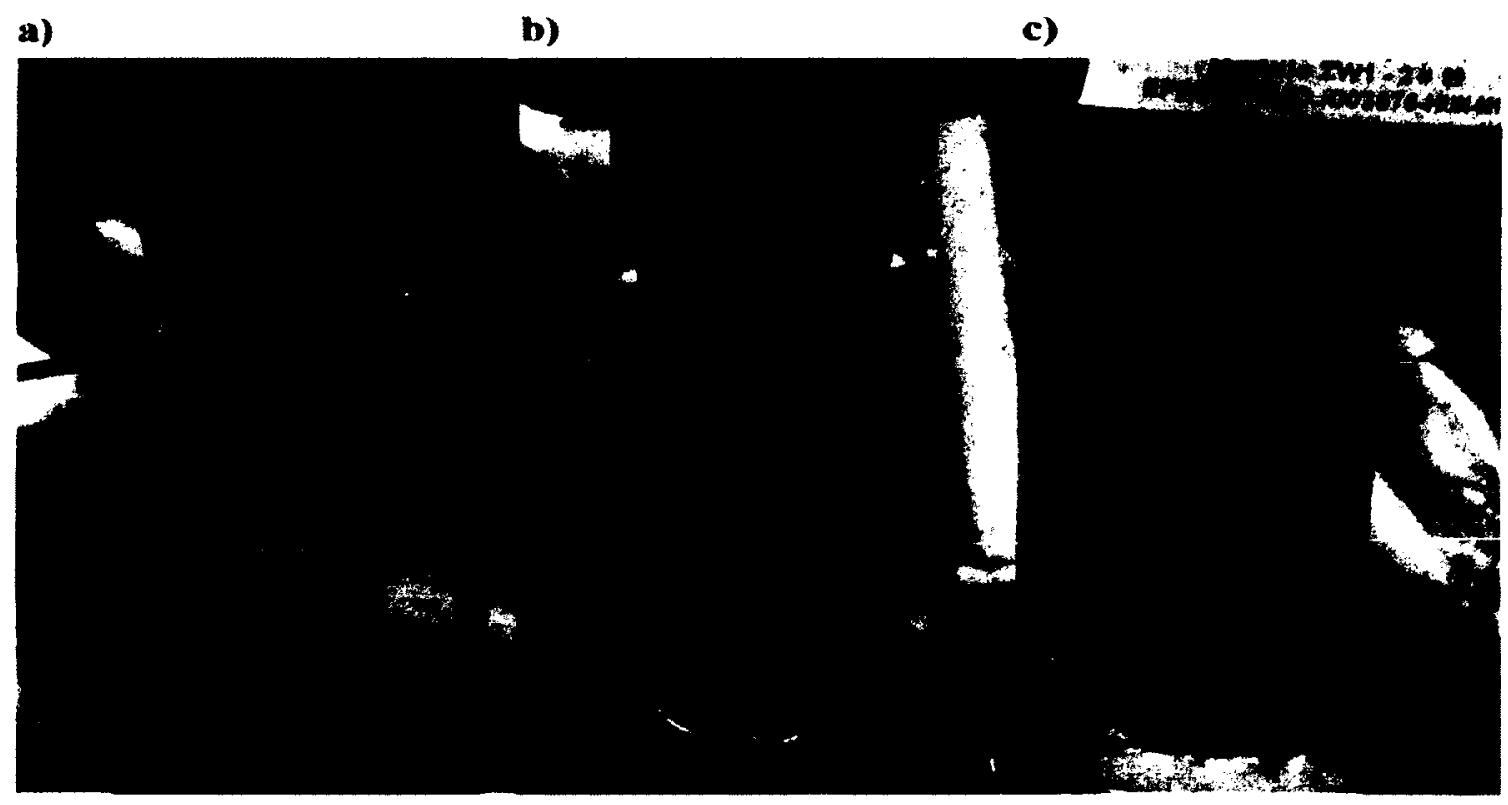




\subsection{Main Effects and Interactions}

Fouling during Main Phase Experiments was measured in terms of TMP logged every 30 seconds during filtration and relaxation periods and every second during backwash periods using the data acquisition program Operation Flux 2.0. The change in TMP at the end of each 5 day long experiment was calculated by taking the difference between the minimum TMP readings recorded in the first and final 15 minutes of the experiment. For experiments that were replicated, the average TMP was used for analysis purposes. These readings shown in APPENDIX L were then inserted into the 4-way ANOVA model table illustrated in Chapter 3 to identify main and interaction effects of the operational parameters on fouling. The 4-way ANOVA model with TMP readings from the Main Phase Experiments can be seen in APPENDIX M. Table 5.2 shows the main and interaction effects determined using the 4-way ANOVA model. Identical main and interaction effects were estimated using least square estimation of regression coefficients for the general linear model representing the $2^{4}$ factorial design of experiments. Brief interpretations of the determined main and interaction effects are included in Table 5.2.

Based on the magnitude of the effects and interaction, the main effect of filtration flux was found to be the most important effect on fouling. With regards to the effects of the cleaning operations on fouling, the main effects of air flow rate and relaxation duration showed a higher level of importance compared to the effect of backwash duration. Due to the low magnitude of the interactions between air flow rate and backwash duration as well as backwash duration and relaxation duration, these interactions were identified as unimportant interactions with minimal effect to fouling. 
Table 5.2 - Main effects and interactions estimated using a 4-way ANOVA model and general linear modeling.

\begin{tabular}{|c|c|c|c|c|}
\hline Effect or Interaction & $\begin{array}{c}\text { Parameters } \\
\text { from ANOVA } \\
\text { Model }\end{array}$ & $\begin{array}{l}\text { Regression } \\
\text { coefficients } \\
\text { for the GLM }\end{array}$ & Value & Interpretation (Rank of Importance) \\
\hline Mean Response: & $\boldsymbol{\mu} \ldots . .$. & $\beta_{0}$ & -15.8 & \\
\hline \multicolumn{5}{|l|}{ Main Effects: } \\
\hline Filtration flux (FF) & $\boldsymbol{\alpha}_{2}$ & $\boldsymbol{\beta}_{1}$ & -9.96 & Most important effect overall. (1) \\
\hline Air flow rate (AFR) & $\boldsymbol{\beta}_{\mathbf{2}}$ & $\boldsymbol{\beta}_{\mathbf{2}}$ & 5.55 & Most important effect by a cleaning operation. (2) \\
\hline Backwash duration (BD) & $\boldsymbol{Y}_{2}$ & $\boldsymbol{\beta}_{\mathbf{3}}$ & 2.19 & Least important effect by a cleaning parameter. ( 8 ) \\
\hline Relaxation duration (RD) & $\delta_{2}$ & $\boldsymbol{\beta}_{4}$ & 4.69 & Fourth most important effect overall. (4) \\
\hline \multicolumn{5}{|l|}{ Two factor interactions: } \\
\hline FF \& AFR & $\boldsymbol{\alpha} \boldsymbol{\beta}_{22}$ & $\boldsymbol{\beta}_{12}$ & 3.34 & Interference of FF on fouling reduction by AFR. (6) \\
\hline FF \& BD & $a \gamma_{22}$ & $\beta_{13}$ & 1.65 & Interference of FF on fouling reduction by BD. (12) \\
\hline FF \& RD & $\alpha \bar{\delta}_{22}$ & $\beta_{14}$ & 4.81 & High interference of FF on fouling reduction by RD. (3) \\
\hline AFR \& BD & $\beta Y_{22}$ & $\boldsymbol{\beta}_{23}$ & 0.08 & Interaction effect almost non-existent. Deemed unimportant. (15) \\
\hline AFR \& RD & $\beta \delta_{22}$ & $\boldsymbol{\beta}_{24}$ & -2.12 & $\begin{array}{l}\text { Possible interference of AFR on fouling reduction by RD or vice versa. } \\
\text { (9) }\end{array}$ \\
\hline$B D \& R D$ & $\mathbf{\gamma} \bar{\delta}_{22}$ & $\boldsymbol{\beta}_{34}$ & -0.76 & $\begin{array}{l}\text { Minor antagonistic interaction between backwash duration and } \\
\text { relaxation duration. Deemed unimportant. (14) }\end{array}$ \\
\hline \multicolumn{5}{|l|}{ Throe factor interactions: } \\
\hline FF, AFR \& BD & $\alpha \beta Y_{222}$ & $\beta_{123}$ & 1.02 & Hard to interpret. Three factor interaction plots needed. (13) \\
\hline FF, AFR \& RD & $\alpha \beta \delta_{222}$ & $\beta_{124}$ & -3.56 & Hard to interpret. Three factor interaction plots needed. (5) \\
\hline FF, BD \& RD & $a y \delta_{222}$ & $\beta_{134}$ & -1.83 & Hard to interpret. Three factor interaction plots needed. (10) \\
\hline$A F R, B D \& R D$ & $\beta Y \delta_{222}$ & $\beta_{234}$ & -1.75 & Hard to interpret. Three factor interaction plots needed. (11) \\
\hline \multicolumn{5}{|l|}{ Four factor interactions: } \\
\hline FF, AFR, BD \& RD & $\alpha \beta \gamma \delta_{2222}$ & $\boldsymbol{\beta}_{\mathbf{1 2 3 4}}$ & -2.48 & $\begin{array}{l}\text { Hard to interpret. Possible synergistic effect between the three physical } \\
\text { cleaning operations and filtration flux. (7) }\end{array}$ \\
\hline
\end{tabular}


Table 5.2 also shows the presence of a number of noticeable important interactions based on the magnitude of the interactions including the two-way interactions between filtration flux and relaxation duration, filtration flux and air flow rate as well as the three-way interaction between filtration flux, air flow rate and relaxation duration. Two-way interactions were interpreted based on the main effects involved in the interactions. For a positive interaction, the interaction was said to be synergistic if the main effects were positive and antagonistic (or interfering) if the main effects were negative (Kutner et al., 2005). Similarly, negative interactions were said to be synergistic if the main effects involved in the interaction were negative and antagonistic if the main effects were positive.

The two-way interaction between filtration flux and relaxation was interpreted as antagonistic such that filtration flux was thought to interfere with the reduction of fouling by relaxation duration. However, the contrary was also speculated with air flow rate aiding in the reduction of fouling caused by filtration flux. Similar contrasting interpretations were made for the interaction between filtration flux and air flow rate. To clearly interpret such results it was necessary to examine the interaction plots associated with these interaction parameters. Interaction plots were particularly essential for complex high order interactions such as the important three-way interaction between filtration flux, air flow rate and relaxation duration where an interpretation could not be made by solely looking at the value of the interaction and main effects. Figure 5.2 to Figure 5.5 show the two-way interactions associated with filtration flux, air flow rate, backwash duration and relaxation duration respectively. 
Figure 5.2 - Two way interactions between Filtration flux (Lo $\rightarrow 19$ LMH, Hi $\rightarrow 38$ LMH) and a) Air flow rate (Lo $\rightarrow 5$ LPM, Hi $\rightarrow 15$ LPM), b) Backwash duration (Lo $\rightarrow 10$ sec., Hi $\rightarrow 20 \mathrm{sec}$.) and c) Relaxation duration (Lo $\rightarrow 5$ min., $\mathrm{Hi} \rightarrow 15$ min.).
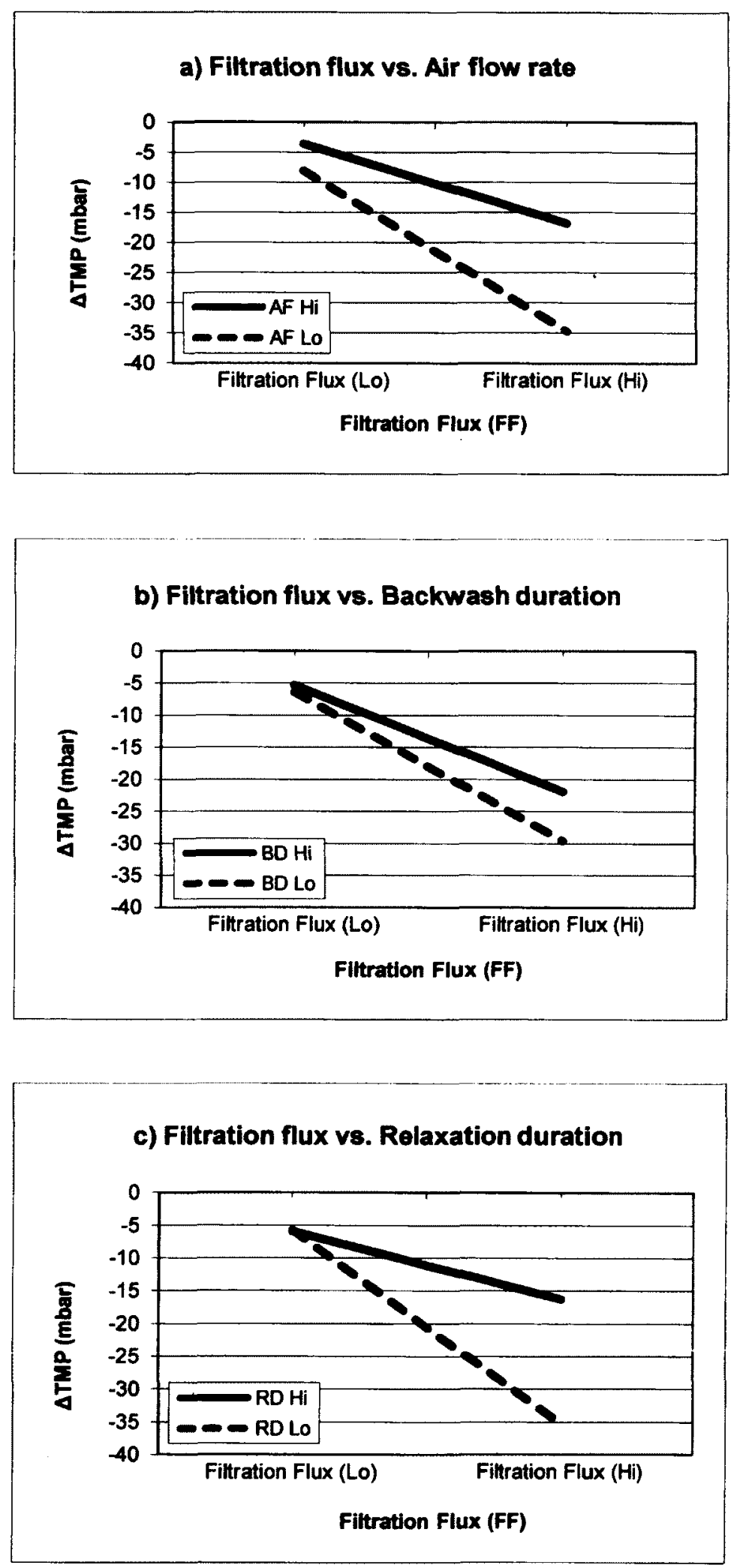
Figure 5.3 - Two way interactions between Air flow rate (Lo $\rightarrow 5 \mathrm{LPM}, \mathrm{Hi} \rightarrow 15 \mathrm{LPM}$ ) and a) Filtration flux (Lo $\rightarrow 19$ LMH, Hi $\rightarrow 38$ LMH), b) Backwash duration (Lo $\rightarrow 10$ sec., Hi $\rightarrow 20$ sec.) and c) Relaxation duration (Lo $\rightarrow 5$ min., Hi $\rightarrow 15$ min.).
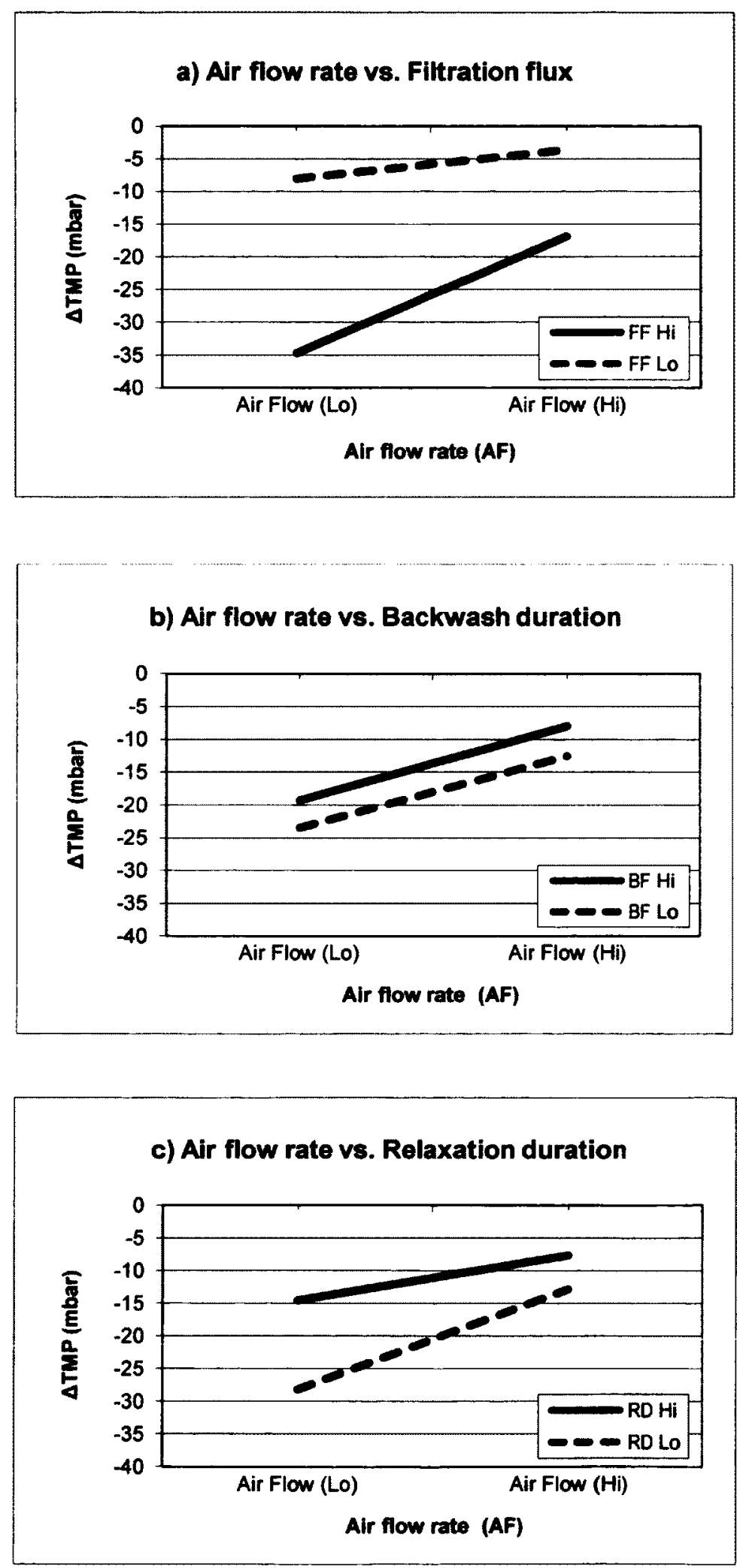
Figure 5.4 - Two way interactions between Backwash duration (Lo $\rightarrow 10$ sec., Hi $\rightarrow 20$ sec.) and a) Filtration flux (Lo $\rightarrow 19 \mathrm{LMH}, \mathrm{Hi} \rightarrow 38 \mathrm{LMH})$, b) Air flow rate (Lo $\rightarrow 5 \mathrm{LPM}, \mathrm{Hi} \rightarrow 15 \mathrm{LPM})$ and c) Relaxation duration (Lo $\rightarrow 5$ min., Hi $\rightarrow 15$ min.).
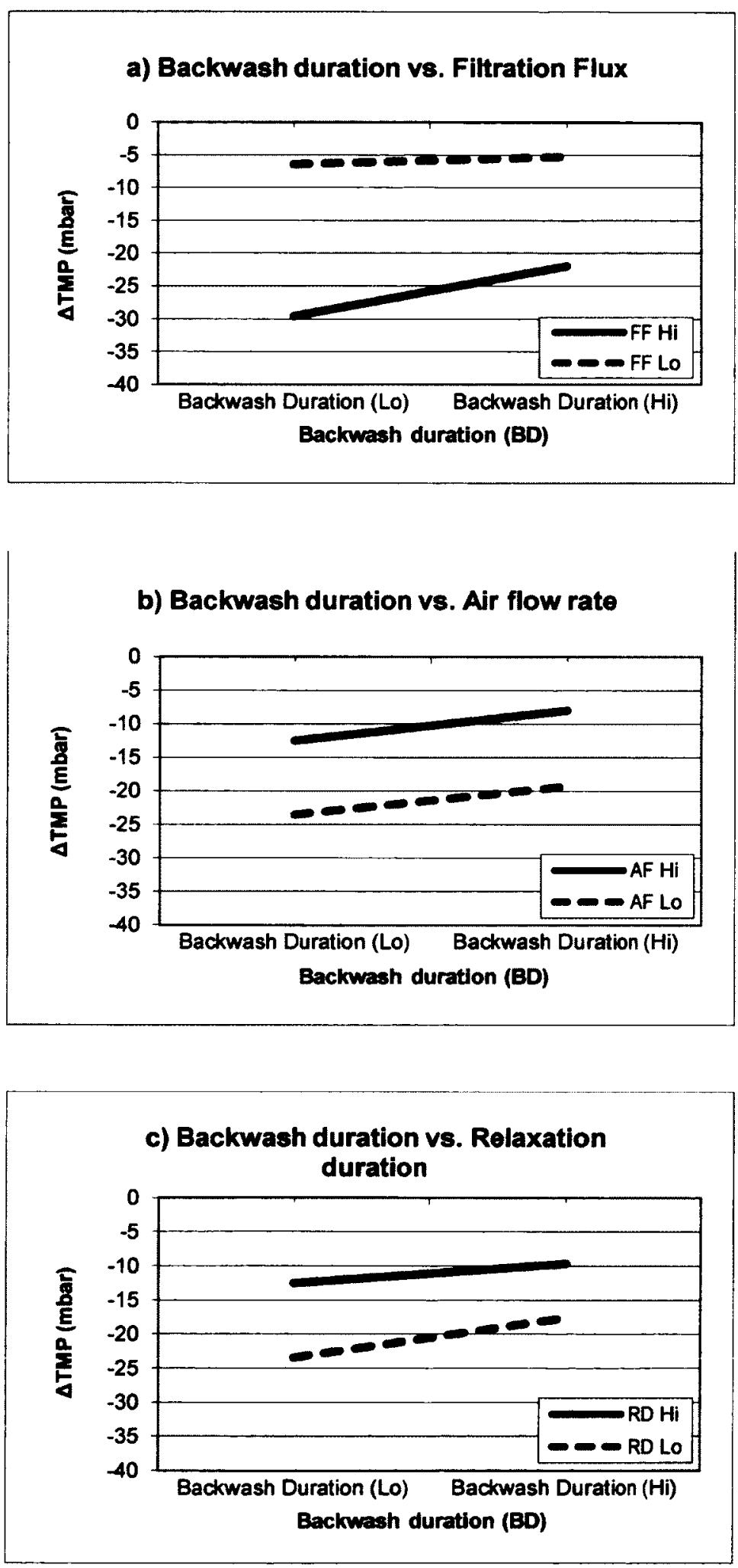
Figure 5.5 - Two way interactions between Relaxation duration $(\mathrm{Lo} \rightarrow 5 \mathrm{~min}$, $\mathrm{Hi} \rightarrow 15 \mathrm{~min}$.) and a) Filtration flux (Lo $\rightarrow 19 \mathrm{LMH}, \mathrm{Hi} \rightarrow 38 \mathrm{LMH}$ ), b) Air flow rate (Lo $\rightarrow 5 \mathrm{LPM}, \mathrm{Hi} \rightarrow 15 \mathrm{LPM}$ ) and c) Backwash duration (Lo $\rightarrow 10$ sec., $\mathrm{Hi} \rightarrow 20$ sec.).
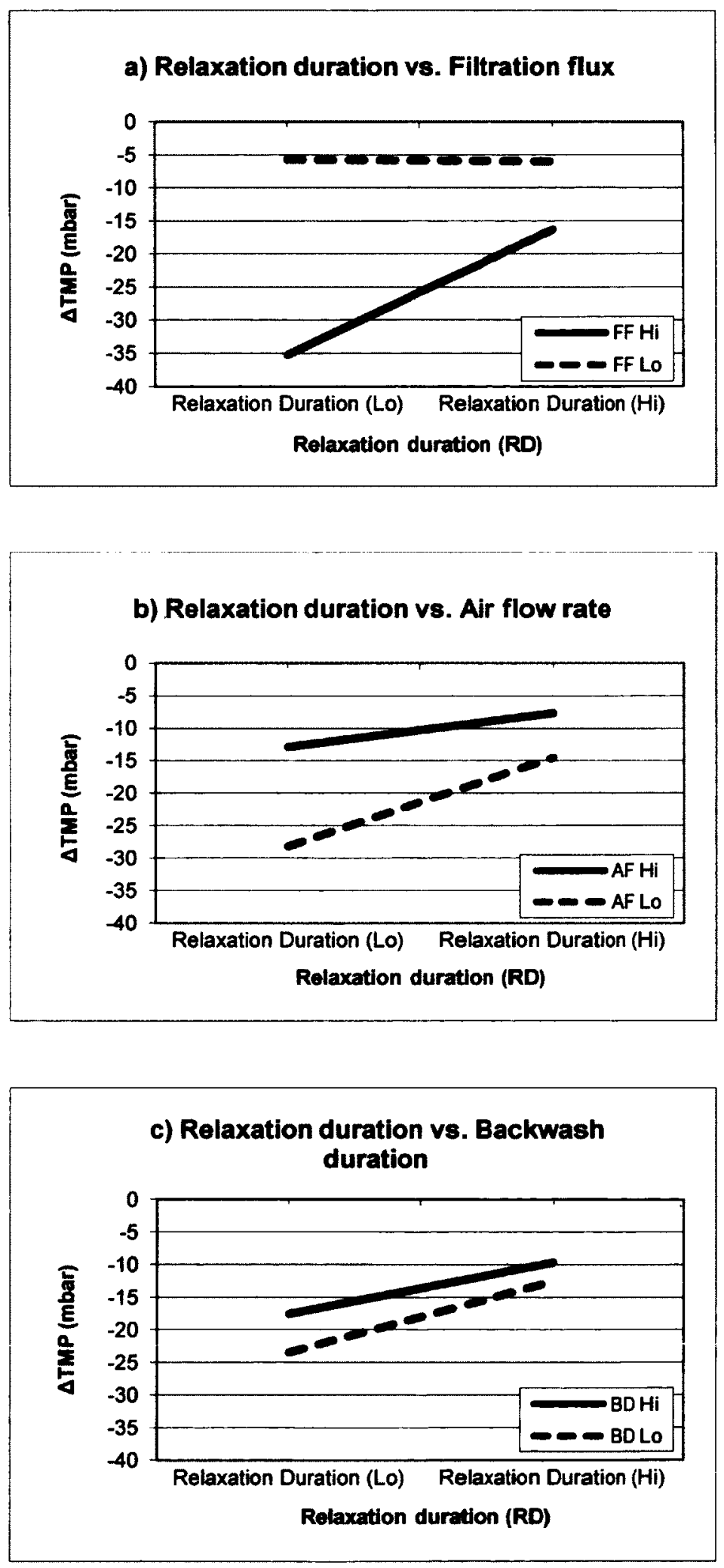
As suspected, a synergistic interaction between filtration flux and air flow rate (Figure 5.2a) existed in which an increase in air flow rate resulted in reduced fouling especially at a higher filtration flux. Interestingly, as also interpreted from the values of the interaction parameter, the antagonistic relationship between filtration flux and air flow rate was also present (Figure 5.3a). An increase in filtration flux was found to greatly increase fouling especially at the low air flow. Similar antagonistic relationships were observed in the cases of two-way interactions of filtration flux with backwash duration and relaxation duration shown in Figure 5.4aand Figure 5.5a respectively. Synergistic relations between filtration flux and backwash duration (Figure 5.2b) as well as filtration flux and relaxation duration (Figure 5.2c) were also present. In both cases, longer durations of backwashing and relaxation duration were found to be effective in fouling reduction only at a high filtration flux. These findings suggested that shorter durations of backwashing and relaxation may be sufficient for fouling attenuation at low filtration fluxes.

The parallel lines shown in Figure 5.3b and almost parallel lines in Figure 5.3c signify the lack of dependence of backwash duration on air flow rate and relaxation duration respectively. These findings confirmed the interpretation that backwash duration combined with either of the other two physical cleaning parameters is not a main contributor to fouling reduction.

Interestingly the interaction parameter between air flow rate and relaxation duration estimated using the ANOVA model (see Table 5.2) suggested that the interaction between the two operation parameters was antagonistic but the main contributor to the effect could not be identified. However, the interaction plots (Figures 
Figure 5.3c and Figure 5.5b of the interaction between air flow rate and relaxation showed that the interaction was synergistic in which an increase in the level of one parameter (air flow rate or relaxation) promoted fouling reduction by the other parameter. Furthermore, the combination of high air flow rate and long relaxation duration resulted in the least fouling.

The difference in the interpretations from the interaction value and the interaction plots between air flow rate and relaxation duration suggested that the interpretation of the two-way interaction between air flow rate and relaxation duration may have been dependent on the interpretation of the three-way interaction between filtration flux, air flow rate and relaxation duration. While higher order interactions are thought to be of minimal significance and usually omitted from the analyses of factorial experiment results, the presence of this three-way interaction of high magnitude prompted the generation of the three-way interaction plots shown in APPENDIX N.

The interaction plot involving filtration flux, air flow rate and relaxation duration showed that the effect of the combination of air flow rate and relaxation duration was highly dependent on filtration flux. High air flow rate with long relaxation durations were found to be efficient for the minimization of fouling at both fluxes. However, in the case of the low filtration flux, shorter relaxation duration at high air flow rate could provide similar results as a longer duration. This was not the case for high filtration flux where shorter relaxation duration was not effective in reducing fouling especially when combined with low air flow rate. Thus the overall antagonistic relationship denoted by the two way interaction between air flow rate and relaxation duration was said to be heavily influenced by the antagonistic effect of filtration flux. 
For the interaction between filtration flux, air flow rate and backwash duration, an antagonistic effect was noticed such that an increase in filtration flux resulted in a decrease in the fouling reduction contribution of air flow rate and backwash duration. Since the analyses of the two way plots indicated that fouling reduction by air flow rate at the two filtration fluxes was not dependent on backwash duration. It is therefore likely that filtration flux was the main contributor to the overall interaction effect.

The interaction between filtration flux, backwash duration and relaxation duration also showed the interference of filtration flux on the effects of the combination of relaxation duration and backwash duration. The interaction plot showed that at either filtration fluxes, the use of long backwash duration with long relaxation duration did not result in any significant decrease in fouling compared to the use of short backwash duration with long relaxation duration. This observation could prove to be beneficial as the use of short backwash durations in combination with relaxation could help lower instantaneous fluxes required to make up for losses in productivity as well as minimize fouling brought about by instantaneous fluxes.

Finally, the three-way interaction between the three cleaning operation parameters showed that the use of the long durations of relaxation and backwashing assisted by high air flow rate was efficient at reducing fouling (APPENDIX N). However when using high air flow rate and long backwash duration, short relaxation duration was sufficient for effective fouling reduction. In contrast, short relaxation durations along with low air flow rate proved to be ineffective in fouling reduction. 


\subsection{Flux Dependent Parameter Effects}

Due to the evident interference of filtration flux on the three cleaning operational parameters, the results from the $2^{4}$ factorial design of experiments were examined based on the two levels of filtration flux (19 LMH and $38 \mathrm{LMH}$ ) to further investigate the behaviours of the three cleaning operations at the two flux levels. While three way interaction plots provided some good insight into ideal combinations of cleaning operational parameters for the reduction of fouling, TMP curves over time were used to draw the interpretations together and provide additional understanding of the working of all four operational parameters collectively.

\subsubsection{Parameter Effects at a Low Filtration Flux}

Figure 5.6 shows the change in TMP employing a low filtration flux (19 LMH) and short backwash duration (10 sec.) while varying air flow rates and relaxation durations. The use of the high air flow rate (15 LPM) was found to be responsible for effective fouling reduction within the group while the long relaxation duration (15 min.) assisted by low air flow (5 LPM) resulted in the least reduction in fouling. While the three-way interaction plot between filtration flux, air flow rate and relaxation duration (APPENDIX N) showed that the short relaxation duration assisted by high air flow was effective for fouling reduction at the low filtration flux, Figure 5.6 showed that the effect of the combination was specific to the accompaniment of short backwash duration in the operational regime. 
Figure 5.6 - TMP change due to low filtration flux (19 LMH) and short backwash duration (10 sec.)

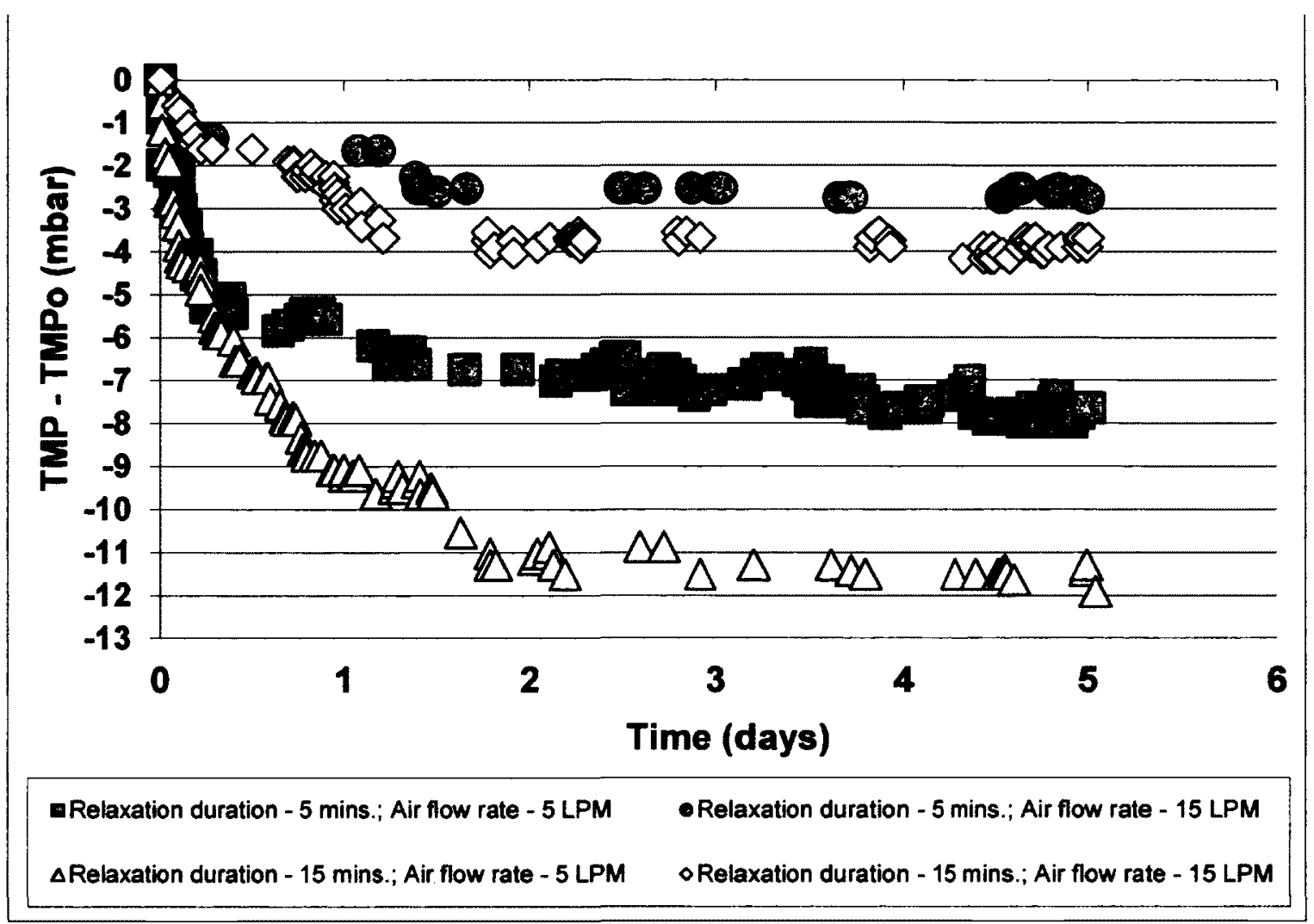

Figure 5.7 shows the case of the longer backwash duration $(20 \mathrm{sec}$.$) and low$ filtration flux. In this case, the longer relaxation duration assisted by high air flow was not only extremely useful for the reduction of fouling but the most effective combination when using low filtration flux and longer backwash duration. On comparing the operational regime resulting in the lowest and highest fouling in Figure 5.7, the effectiveness of the combinations of high air flow rate and long backwash durations was found to be dependent on the long relaxation duration which was in contrast to the adequacy of short relaxation duration with long backwash durations and high air flow interpreted in the three-way interaction plot of the three cleaning parameters. 
Figure 5.7 - TMP change due to low filtration flux (19 LMH) and long backwash duration (20 sec.)

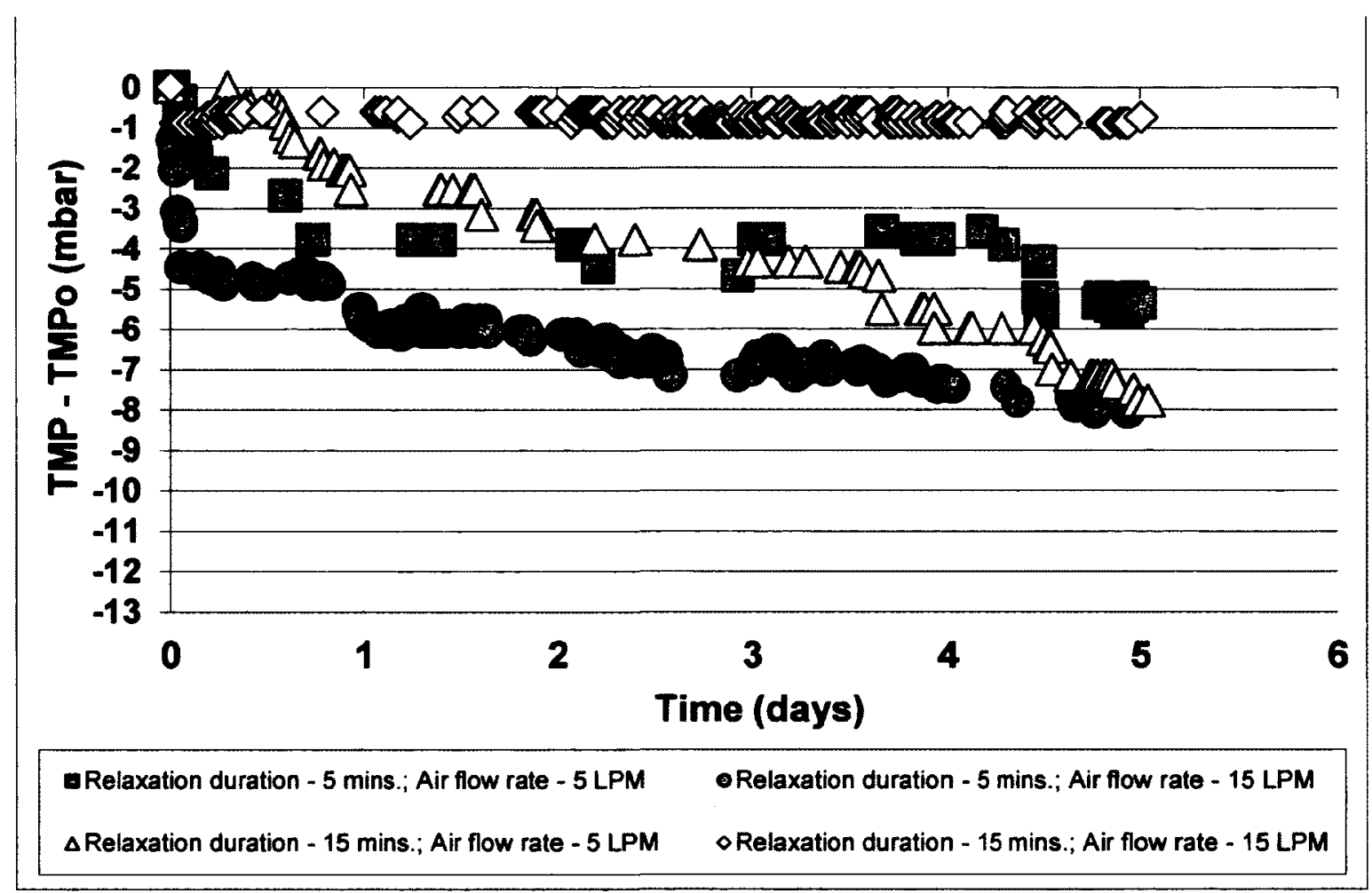

The treatment combinations resulting in the lowest and highest fouling from Figure 5.6 and Figure 5.7 were combined in Figure 5.8 to identify the most and least efficient combination for fouling reduction at the lower filtration flux. While the combination of high air flow rate with long backwash and relaxation durations was found to be the most efficient treatment at the low filtration flux, such a cleaning regime may not be practical due to losses in permeate productivity associated with the long backwash and relaxation periods. Thus, based on the TMP curve in Figure 5.8 as well as results from the interaction plots, the combination of high flow rate with short durations of backwashing and relaxation was identified as the best practical combination for fouling attenuation at the low flux. The importance of air flow rate on fouling attenuation at the low flux was also highlighted in Figure 5.8 as a reduction in air flow rate while using the 
short backwash and relaxation durations was identified as the most inefficient combination for fouling reduction.

Figure 5.8 - Identification of the most and least efficient cleaning regimes for low filtration flux operations (19 LMH).

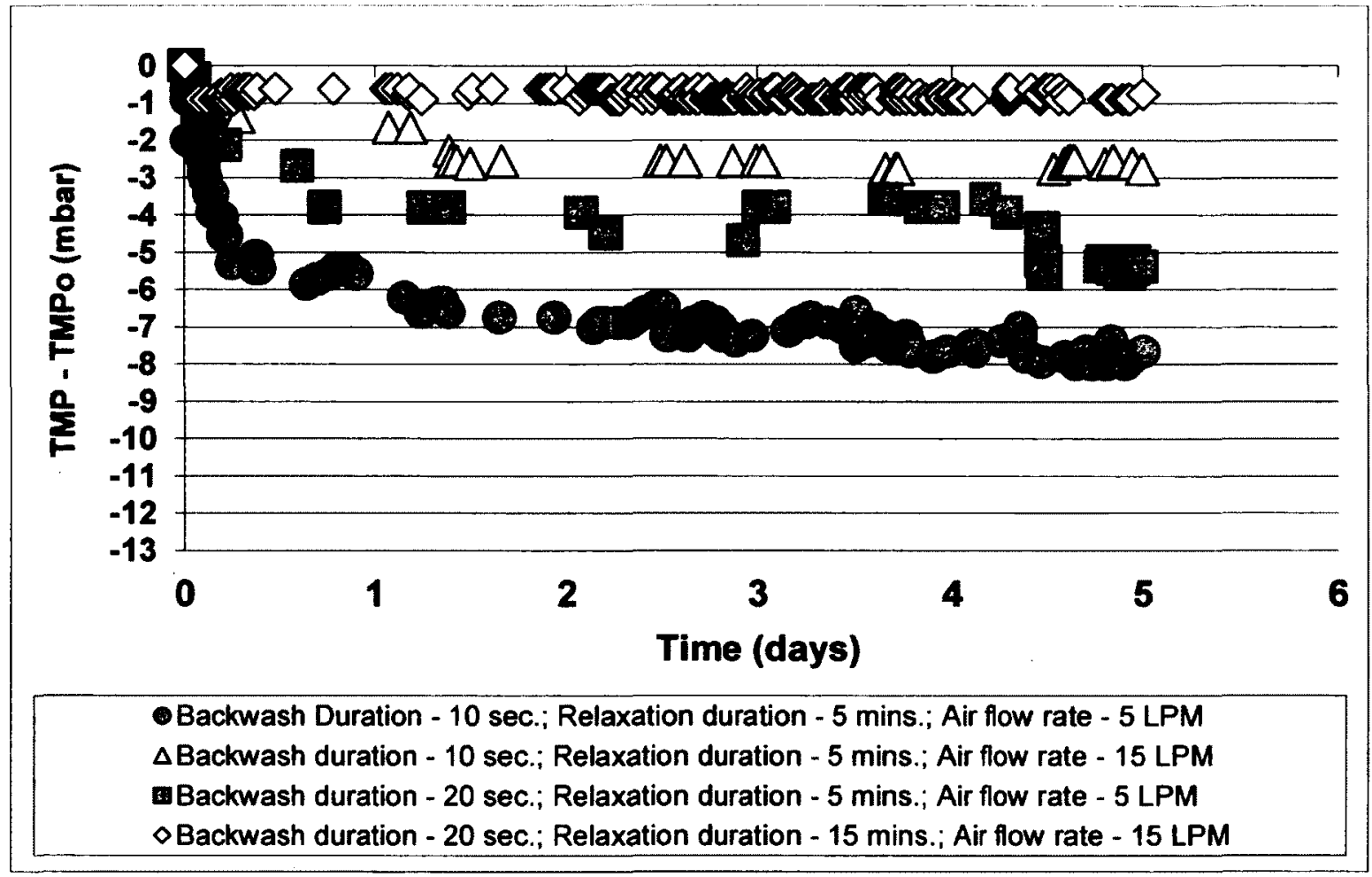

\subsubsection{Parameter Effects at a High Filtration Flux}

Figure 5.9 shows the change in TMP observed for Main Phase Experiments employing a high filtration flux (38 LMH) and short backwash duration (10 sec.) while varying the air flow rates and relaxation durations As interpreted in the three way interaction plot between filtration flux, air flow rate and backwash duration and the plot between filtration flux, backwash duration and relaxation duration, a high relaxation duration and high air flow rate were found to be ideal for fouling reduction when using a 
short backwash duration and high filtration flux. Consequentially, decreasing the relaxation duration and air flow rate proved to be ineffective in reducing fouling at the high filtration flux.

Figure 5.9- TMP change due to high filtration flux ( $38 \mathrm{LMH})$ and short backwash duration (10 sec.)

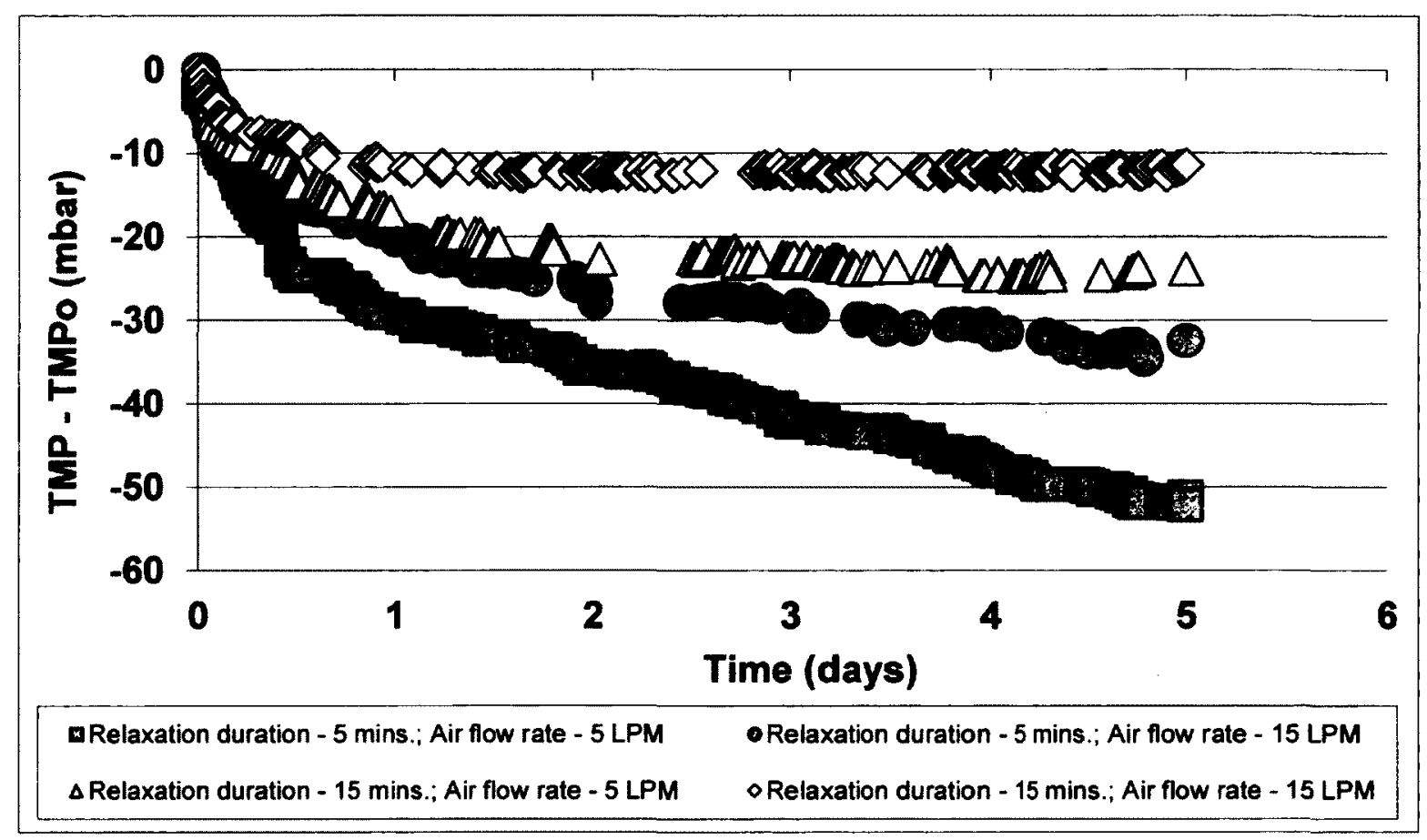

As with the treatment combinations involving high filtration flux and short backwashing, the use of short relaxation duration and low air flow rate with long backwash durations and high filtration flux was also inefficient at reducing fouling (Figure 5.10). It was also found that when using the higher filtration flux and long backwash duration, fouling was significantly reduced by either using a high air flow for scouring, long relaxation durations or a combination of both operations. These results suggested that the long backwash duration did not have an effect on fouling at the high filtration flux. 
Figure 5.10 - TMP change due to high filtration flux (38 LMH) and long backwash duration (20 sec.) (Note: Curves for combinations involving 15 min. relaxation durations overlap)

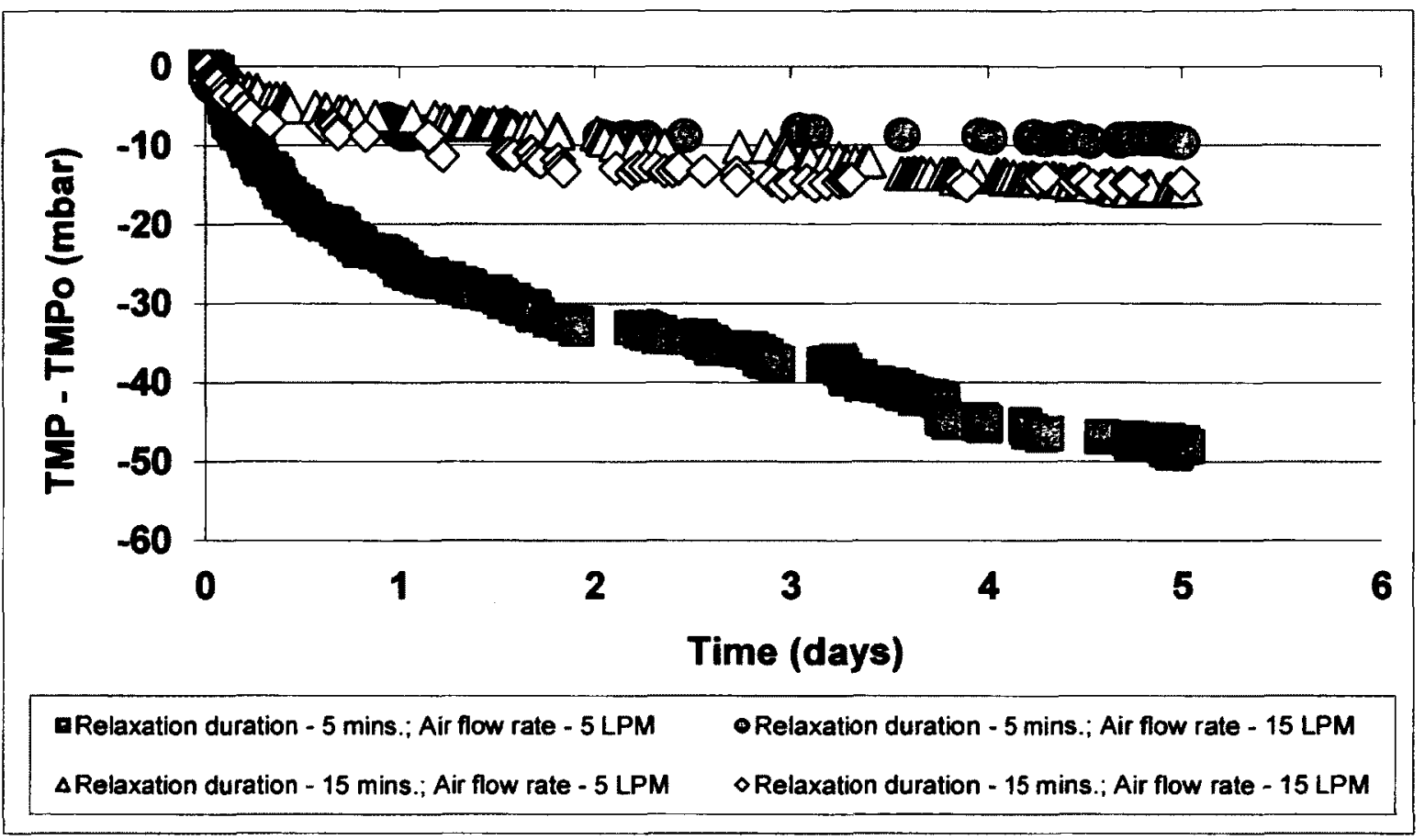

The treatment combinations resulting in the lowest and highest fouling from Figure 5.9 and Figure 5.10 were combined in Figure 5.11 to analyse the effect of backwash duration on fouling as well as identify the most and least efficient combination for the reduction of fouling at the high filtration flux. While Figure 5.10 suggested a noneffect of long backwash duration on fouling at the high filtration flux, an effect of longer backwash duration was evident in Figure 5.11 such that the longer backwash duration contributed to lesser fouling reduction than the shorter duration. However this effect of backwash duration seemed rather minor when compared to the significant effect of air flow rate on fouling and thus provided an explanation of the low overall effect of backwash duration estimated by the ANOVA model.

Two options for efficient fouling reduction at the high flow rate were identified. Both options involved the use of high air flow rate assisting either a short backwash 
duration with long relaxation duration or long backwash duration with short relaxation duration. While both options provide similar reductions in fouling, the use of long backwash duration and short relaxation duration would be more practical in terms of minimizing losses in permeate productivity. For the purpose of reducing fouling, the use of short relaxation duration assisted by low air flow rate should be avoided.

Figure 5.11- Identification of the most and least efficient cleaning regimes for high filtration flux operations (38 LMH).

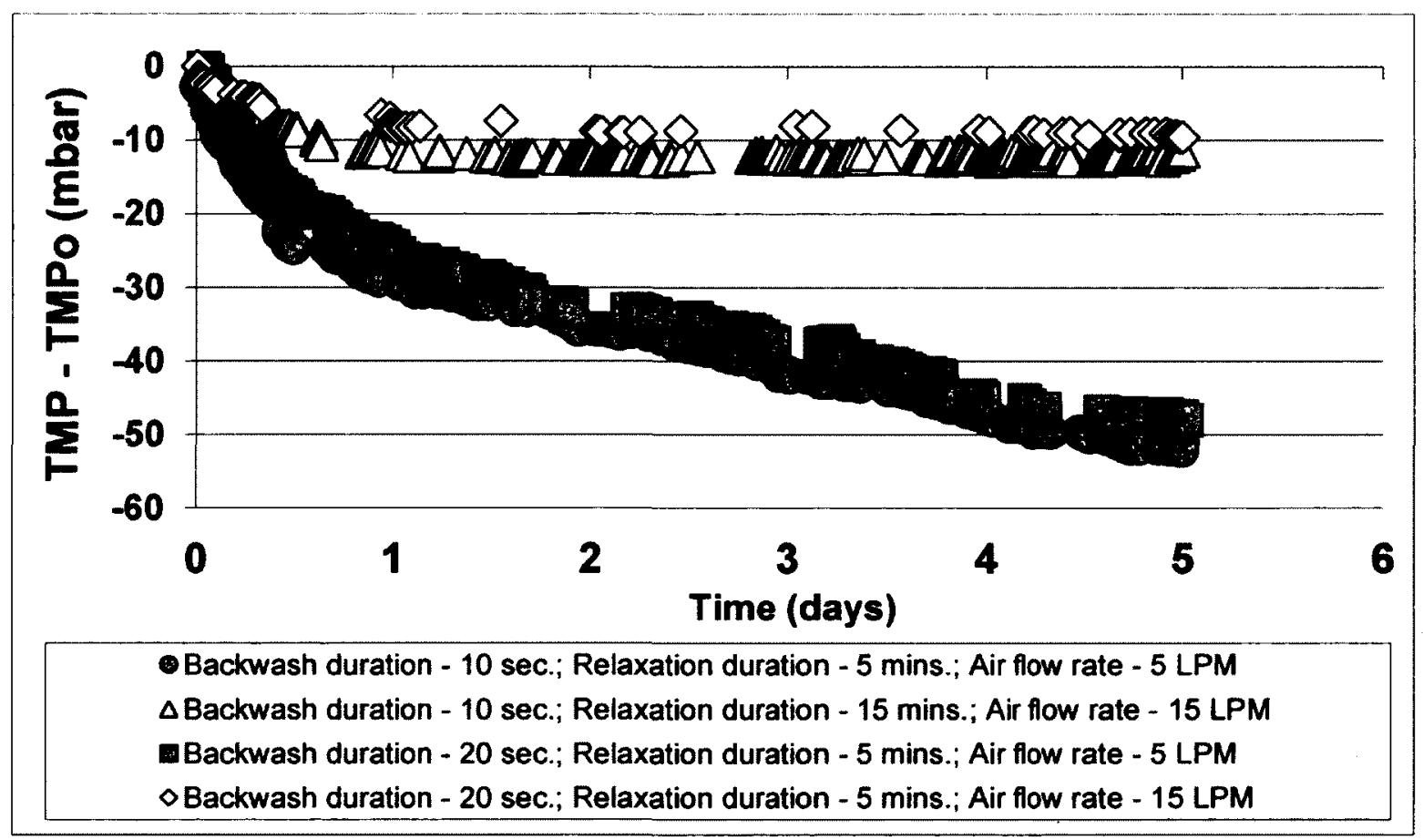

\subsection{Important Effects}

Based on the magnitude of the effects and interactions determined by the ANOVA model and general linear modelling, most effects and interactions were considered important with the exception of the two-way interactions of backwash duration with air flow rate and relaxation duration. Two-way interaction plots confirmed 
that these interactions were not major contributors to fouling reduction. The interaction plots also speculated on the importance of other interactions such as the three-way interaction between filtration flux, air flow rate and backwashing as well as the two-way interaction between filtration flux and backwash duration. To further identify the importance of effects and interactions on fouling reduction, a Sum of Squares of Residuals (SSR) comparison was performed (methodology as illustrated in CHAPTER 3 Section 3.5.2).

Figure 5.12 shows the SSR obtained for reduced models as a result of the exclusion of individual regression coefficients and the corresponding variables from the GLM representing the $2^{4}$ factorial design of experiments. Reduced models with SSR greater the zero (SSR of the original GLM) were identified as important effects such that the larger the determined SSR the greater the importance of the effect. As expected, the effect of filtration flux of fouling was identified as the most important effect followed by the main effect of air flow rate, the two way interaction between filtration flux and relaxation duration and the main effect of relaxation duration. In addition to these four most important effects, the three-way interaction between filtration flux, air flow rate and relaxation duration and the two-way interaction between filtration flux and air flow rate were also identified as important effects. 
Figure 5.12 - Sum of Squares of Residuals comparison for the identification of important effects

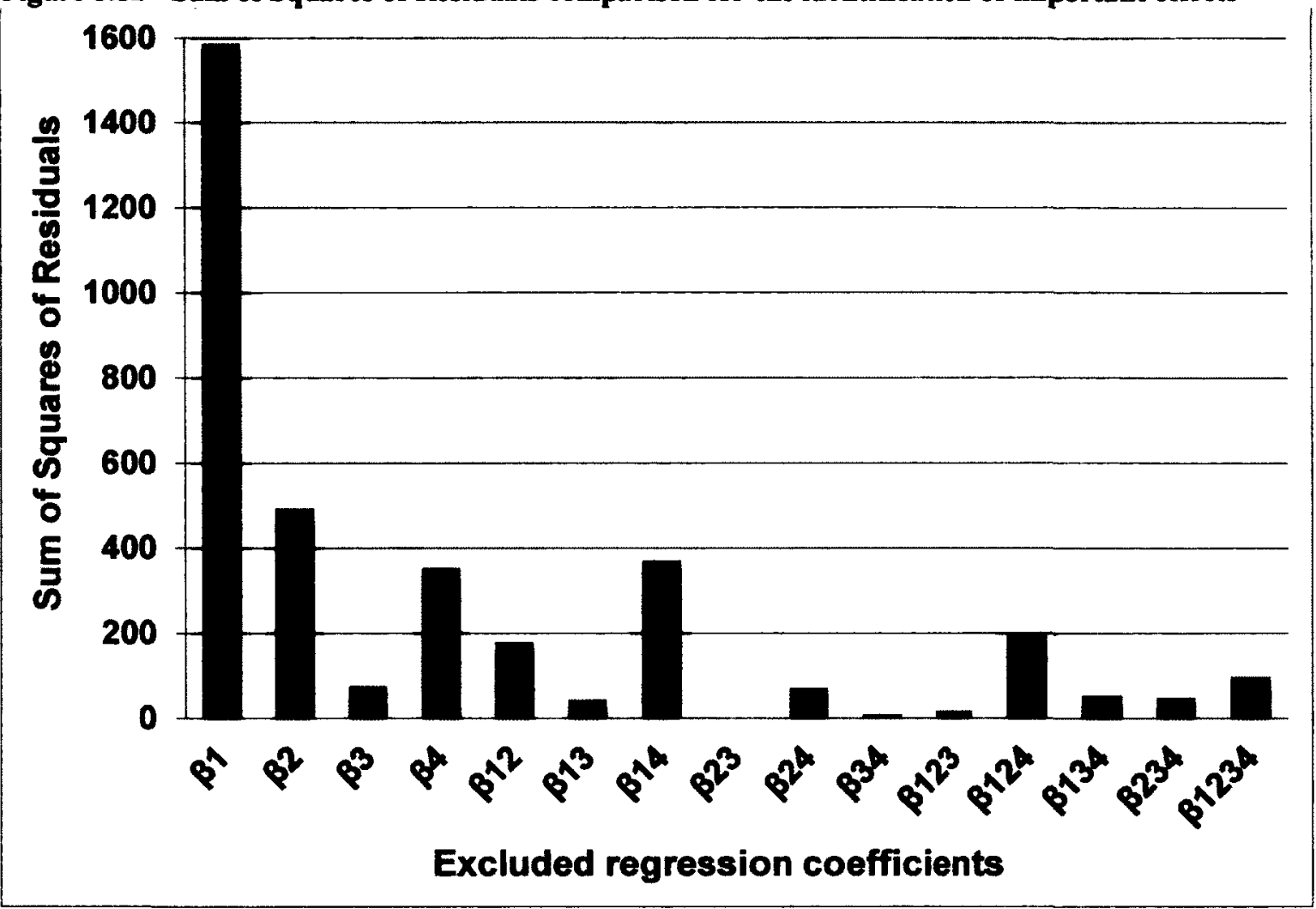

For a more statistically sound identification of important effects, stepwise regression was performed using $\alpha$ values of 0.10 and 0.15 for the acceptance and rejection of effects respectively. The statistical program JMP 9.0 by SAS was used to carry out the stepwise regression during which effects and interactions shown in Table 5.3 were identified as important effects. These effects which significantly contributed to fouling were used for the development of a time based statistical model for the prediction of fouling over time (Discussed in further detail in CHAPTER 6). The step wise regression carried out by JMP 9.0 can be seen in APPENDIX O.

All effects and interactions involving backwash duration were eliminated from the model showing the unimportance of the effect of backwash duration on fouling reduction 
by the cleaning regime employed in this study. However, this elimination by stepwise linear regression only shows the ineffectiveness of backwash duration on fouling and should not be mistaken for the effect of the operation of backwashing on fouling.

Table 5.3 - Important effects identified by stepwise linear regression

\begin{tabular}{|c|c|c|}
\hline $\begin{array}{l}\text { Important Effect or } \\
\text { Interaction }\end{array}$ & $\begin{array}{c}\text { Parameters } \\
\text { from } \\
\text { ANOVA } \\
\text { Model } \\
\end{array}$ & $\begin{array}{c}\text { Regression } \\
\text { coefficients for } \\
\text { the reduced } \\
\text { GLM }\end{array}$ \\
\hline Mean Response: & p.... & $\beta_{0}$ \\
\hline \multicolumn{3}{|l|}{ Main Effects: } \\
\hline Filtration flux (FF) & $\mathbf{\alpha}_{2}$ & $\boldsymbol{\beta}_{1}$ \\
\hline Air flow rate (AFR) & $\boldsymbol{\beta}_{2}$ & $\boldsymbol{\beta}_{\mathbf{2}}$ \\
\hline Relaxation duration (RD) & $\delta_{2}$ & $\boldsymbol{\beta}_{4}$ \\
\hline \multicolumn{3}{|l|}{ Two factor interactions: } \\
\hline FF \& AFR & $\alpha \beta_{22}$ & $\beta_{12}$ \\
\hline FF \& RD & $\alpha \delta_{22}$ & $\beta_{14}$ \\
\hline AFR \& RD & $\boldsymbol{\beta}_{\mathbf{2 2}}$ & $\boldsymbol{\beta}_{24}$ \\
\hline \multicolumn{3}{|l|}{ Three factor interactions: } \\
\hline$F F, A F R \& R D$ & $\boldsymbol{\alpha} \boldsymbol{\beta} \boldsymbol{\delta}_{222}$ & $\beta_{124}$ \\
\hline
\end{tabular}

\subsection{Water Quality Assessment}

During all $2^{4}$ factorial experiments performed in the Main Phase Experiments, samples were taken from the feed tank, reactor tank and permeate and tested for $\mathrm{pH}$, turbidity and COD to monitor for any significant changes in water quality that may aid or impede fouling. While the $\mathrm{pH}$ and turbidity were measured daily, the COD of the three 
solutions was tested at the start and end of each experiment as well as at the end of day two of the five day experiments. The permeate flow was also measured at the start and end of each experiment to identify any loss of productivity of the membrane due to membrane fouling. Table 5.4 shows the $\mathrm{pH}$, turbidity and COD recorded in the reactor tank for the two least and most fouling experiments from the $2^{4}$ factorial design. The complete set of results from water quality analysis for all experiments employing a low and high filtration flux can be seen in APENDICES $P$ and $Q$ respectively.

No significant or unexpected changes to the water quality were observed during experiments. In all cases, the occurrence of foulant accumulation in the reactor tank was noticed. However, as seen in the Preliminary Phase Study experiments employing a continuous flow operation with $\mathbf{8 0 \%}$ recovery, once the initial increase in water quality parameters due to accumulation of foulants occurred, the overall water quality of the solution in the reactor tank remained fairly consistent over the 5 day long experiments.

An assessment of the permeate water quality showed that a high quality of water was produced during all the Main Phase Experiments. A comparison of the permeate flow at the two filtration fluxes showed that productivity of the membrane decreased over time when using a high filtration flux especially for experiments where high fouling was noticed. This was not the case for the low filtration flux where productivity was consistent over time. 
Table 5.4 - Water quality assessment of least (Exp. 3 and 15) and most (Exp. 2 and 6) fouling experiments.

\begin{tabular}{|c|c|c|c|c|c|c|c|}
\hline Sample & $\mathrm{pH}$ & $\begin{array}{c}\text { Turbidity } \\
\text { (NTU) }\end{array}$ & $\begin{array}{c}\text { COD } \\
(\mathrm{mg} / \\
\mathrm{L})\end{array}$ & Sample & $\mathrm{pH}$ & $\begin{array}{l}\text { Turbidity } \\
\text { (NTU) }\end{array}$ & $\begin{array}{c}\text { COD } \\
(\mathrm{mg} / \\
\mathrm{L})\end{array}$ \\
\hline \multicolumn{4}{|c|}{ Experiment 3 - $\| F F ; \Uparrow A F ; \Downarrow B D ; \Downarrow R D$} & \multicolumn{4}{|c|}{ Experiment 2 - $\Uparrow F F ; \Downarrow A F ; \Downarrow B D ; \Downarrow R D$} \\
\hline $\begin{array}{l}\text { Reactor } \\
\text { (Initial) }\end{array}$ & 7.74 & 30.23 & 18.50 & $\begin{array}{c}\text { Reactor } \\
\text { (Initial) }\end{array}$ & 7.77 & 31.90 & 17.50 \\
\hline $\begin{array}{c}\text { Reactor } \\
\text { (1 day) }\end{array}$ & 7.73 & 83.17 & - & $\begin{array}{l}\text { Reactor } \\
\text { (1 day) }\end{array}$ & 7.83 & 73.90 & - \\
\hline $\begin{array}{l}\text { Reactor } \\
\text { ( } 2 \text { days) }\end{array}$ & 7.78 & 107.0 & 21.50 & $\begin{array}{l}\text { Reactor } \\
\text { (2 days) }\end{array}$ & 7.79 & 65.98 & 35.00 \\
\hline $\begin{array}{l}\text { Reactor } \\
\text { ( } 3 \text { days) }\end{array}$ & 7.80 & 115.0 & - & $\begin{array}{l}\text { Reactor } \\
\text { ( } 3 \text { days) }\end{array}$ & 7.75 & 68.07 & - \\
\hline $\begin{array}{l}\text { Reactor } \\
\text { (4 days) }\end{array}$ & 7.81 & 108.0 & - & $\begin{array}{l}\text { Reactor } \\
\text { (4 days) }\end{array}$ & 7.71 & 64.90 & - \\
\hline $\begin{array}{l}\text { Reactor } \\
\text { (5 days) }\end{array}$ & 7.89 & 114.5 & 38.00 & $\begin{array}{l}\text { Reactor } \\
\text { (5 days) }\end{array}$ & 7.62 & 62.37 & 31.00 \\
\hline \multicolumn{4}{|c|}{ Experiment $15-\Downarrow F F ; \Uparrow A F ; \pi B D ; \pi R D$} & \multicolumn{4}{|c|}{ Experiment 6 - $\mathbb{F} F F ; \| A F ; \pi B D ; \Downarrow R D$} \\
\hline $\begin{array}{c}\text { Reactor } \\
\text { (Initial) }\end{array}$ & 7.81 & 33.03 & 21.50 & $\begin{array}{l}\text { Reactor } \\
\text { (Initial) }\end{array}$ & 7.76 & 30.83 & 21.00 \\
\hline $\begin{array}{c}\text { Reactor } \\
\text { (1 day) }\end{array}$ & 7.79 & 81.33 & - & $\begin{array}{c}\text { Reactor } \\
\text { (1 day) }\end{array}$ & 7.72 & 89.07 & - \\
\hline $\begin{array}{l}\text { Reactor } \\
\text { (2 days) }\end{array}$ & 7.74 & 92.83 & 24.50 & $\begin{array}{l}\text { Reactor } \\
\text { (2 days) }\end{array}$ & 7.73 & 75.53 & 28.50 \\
\hline $\begin{array}{l}\text { Reactor } \\
\text { (3 days) }\end{array}$ & 7.74 & 102.6 & - & $\begin{array}{l}\text { Reactor } \\
\text { (3 days) }\end{array}$ & 7.70 & 70.97 & - \\
\hline $\begin{array}{l}\text { Reactor } \\
\text { (4 days) }\end{array}$ & 7.76 & 95.27 & - & $\begin{array}{l}\text { Reactor } \\
\text { (4 days) }\end{array}$ & - & - & - \\
\hline $\begin{array}{l}\text { Reactor } \\
\text { (5 days) }\end{array}$ & 7.79 & 112.3 & 44.00 & $\begin{array}{l}\text { Reactor } \\
\text { (5 days) }\end{array}$ & 7.75 & 86.70 & 31.50 \\
\hline
\end{tabular}




\subsection{Cleaning Operation Exclusion Tests}

Cleaning operation exclusion tests were performed to study the fouling effect of the exclusion of individual cleaning operations from the typical operational regime used in the $2^{4}$ factorial design of experiments. Figure 5.13 compares the effect of excluding one cleaning operation at a time from the least effective physical cleaning regime (Air flow rate - 5 LPM; Backwash duration - 10 sec.; Relaxation duration -5 min.) used in the $2^{4}$ factorial design. This experiment was chosen for the exclusion test as it was thought that the results would be sufficiently pronounced (i.e. high fouling) for easier interpretation compared to the results from an experiment which had less fouling associated with it.

Figure 5.13 - Effect of cleaning operation exclusion under high fouling conditions (Filtration Flux $\rightarrow$ 38 LMH).

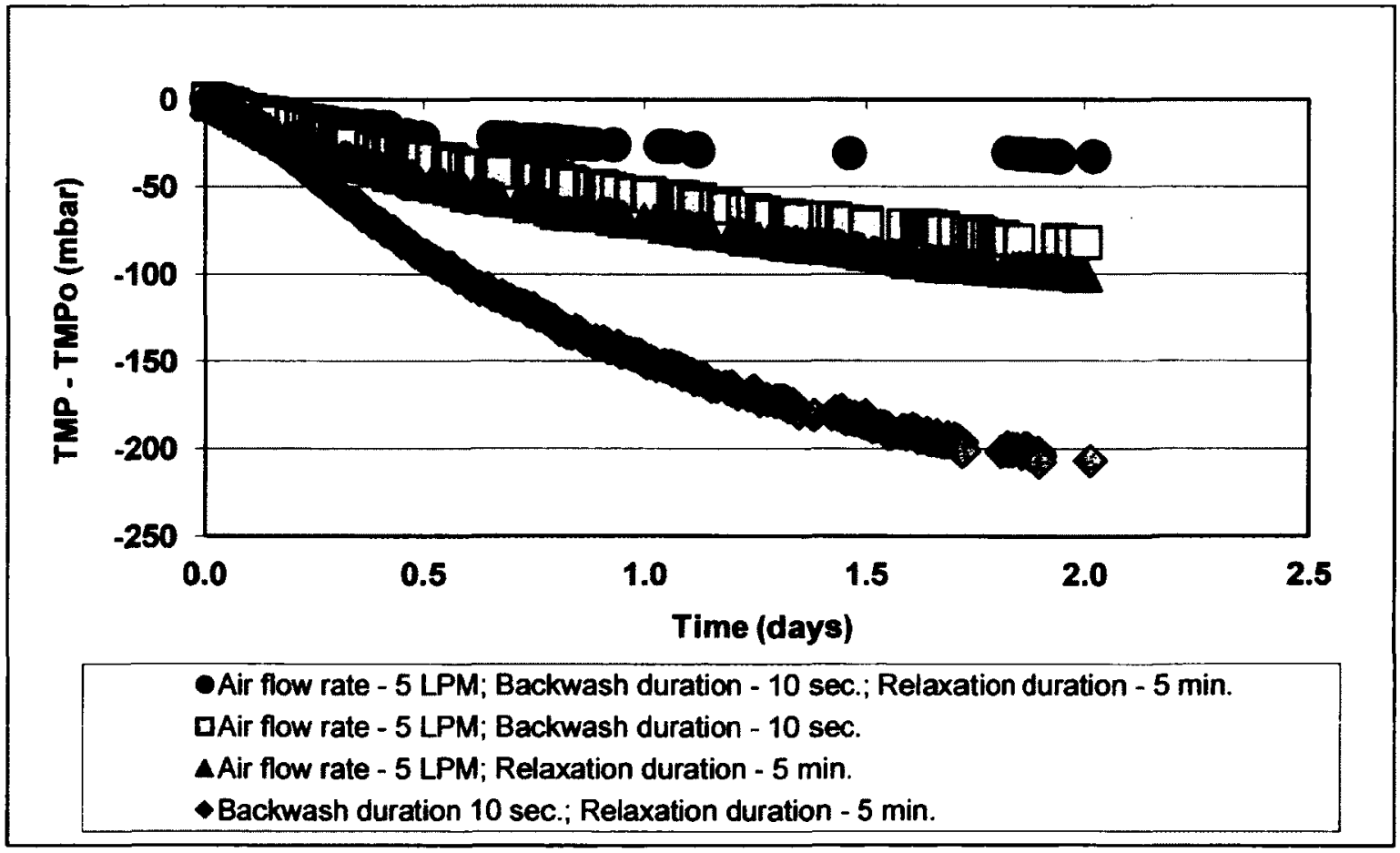


The baseline for comparison in these tests was the combination of the three cleaning operations. As can be clearly seen in Figure 5.13, airflow rate was more essential for fouling reduction than either of the other two parameters. This was in agreement with results from the full factorial experiments and helped to confirm the earlier results. This efficiency was attributed to the greater removal of foulants off the membrane surface compared to the other cleaning regimes employed in the fractional $2^{3}$ factorial experiments as can be observed by examining Figure 5.14. Similar to findings reported by Ye et al. (2011), the use of air assisted backwashing was slightly more efficient in fouling reduction than the use of air assisted relaxation (Figure 5.13) while similar levels of surface fouling were observed for both treatments (Figure 5.14). However, the use of air assisted backwashing and air assisted relaxation was not as effective in fouling reduction as the combination of the three operations and resulted in fouling approximately 3 times greater than that observed using the combination of all three operations.

While results from the $2^{4}$ factorial experiments showed the effect of backwashing duration was insignificant, the 3 fold difference observed when comparing the fouling due to air assisted relaxation and the combination of all three operation demonstrates the importance of backwashing in the three operation cleaning regime. This observation suggests the need for testing other operational parameters of backwashing such as backwashing frequency or backwash strength in future fouling studies. As in the case of the importance of including backwashing in the combination of cleaning operations, the importance of including relaxation was noticed when comparing the fouling due to air assisted backwashing and the combination of the three operations. 
Figure 5.14 - Membrane surface conditions due to cleaning operation exclusion tests (From left to right - With air scouring, backwashing and relaxation (5 days); Without relaxation (2 days); Without backwashing (2 days); Without air scouring (2 days))

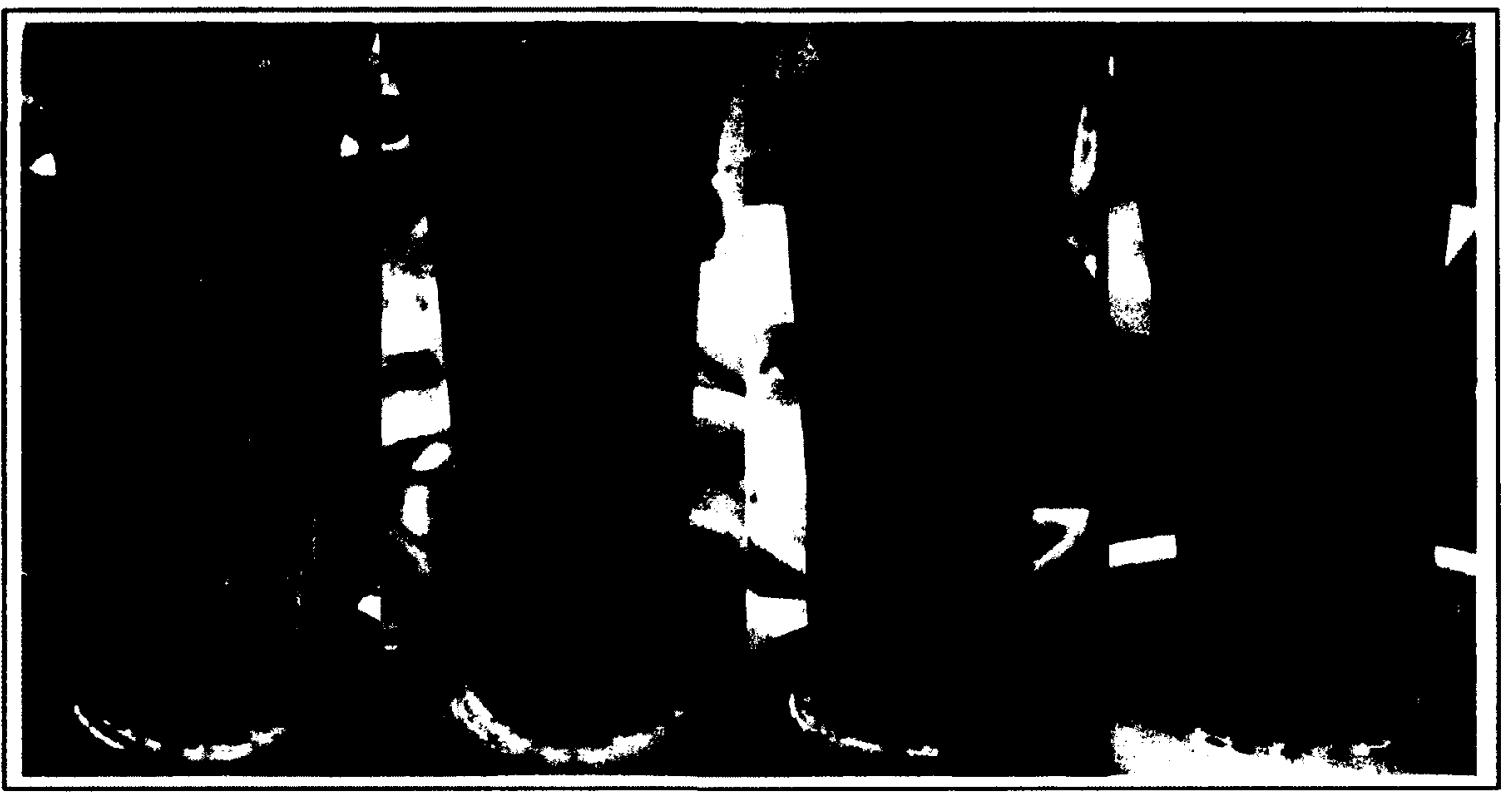

The exclusion of air scouring from the operational regime was found to be extremely crucial as the treatment resulted in a 6 fold increase in fouling compared to the use of the combination of all three cleaning operations (Figure 5.13). This result not only showed the importance of air scouring on fouling reduction but also signified the dominance of the fouling reduction mechanism of air scouring in comparison to relaxation and backwashing.

Table 5.5 shows the changes in water quality of solution in the reactor tank as well as permeate noticed as a result of the respective exclusion of the three cleaning operations from the operational regime. The most noticeable change in water quality was noticed for the experiments which excluded the use of air scouring. Interestingly, this experiment had a significant decrease in Turbidity and COD in the reactor tank which was attributed to the accumulation of the foulants on the membrane surface as seen in 
Figure 5.14. This coincides with the higher level fouling noted for this experimental run. These results show that the use of backwashing and relaxation alone was inefficient in removing foulants off the surface and air scouring was necessary to complete the fouling removal process when utilizing relaxation and backwashing. As expected, the high fouling due to the exclusion of air scouring resulted in a significant loss of permeate production over time.

Table 5.5 - Changes in water quality parameters noticed during cleaning operation exclusion tests

\begin{tabular}{|c|c|c|c|}
\hline Solution & $\begin{array}{c}\text { Turbidity } \\
\text { (NTU) }\end{array}$ & $\begin{array}{c}\text { COD } \\
(\mathrm{mg} / \mathrm{L})\end{array}$ & $\begin{array}{c}\text { Flow } \\
\text { rate } \\
(\mathrm{mL} / \mathrm{min})\end{array}$ \\
\hline \multicolumn{4}{|c|}{ W/o backwashing } \\
\hline Reactor (initial) & 30.2 & 21.0 & - \\
\hline Reactor (24 hrs.) & 69.4 & - & - \\
\hline Reactor (48 hrs.) & 57.2 & 32.5 & - \\
\hline Permeate (initial) & 0.00 & 4 & 30 \\
\hline Permeate (24 hrs.) & 0.00 & - & 29 \\
\hline Permeate (48 hrs.) & 0.00 & 0 & 28 \\
\hline \multicolumn{4}{|c|}{ W/o relaxation } \\
\hline Reactor (initial) & 32.5 & 20.0 & - \\
\hline Reactor (24 hrs.) & 81.8 & - & - \\
\hline Reactor (48 hrs.) & 75.5 & 34 & - \\
\hline Permeate (initial) & 0.02 & 3.5 & 30 \\
\hline Permeate (24 hrs.) & 0.02 & - & 29 \\
\hline Permeate (48 hrs.) & 0.01 & 1.0 & 29 \\
\hline \multicolumn{4}{|c|}{ W/o air scouring } \\
\hline Reactor (initial) & 30.5 & 21.5 & - \\
\hline Reactor (24 hrs.) & 13.4 & - & - \\
\hline Reactor (48 hrs.) & 9.1 & 6.5 & - \\
\hline Permeate (initial) & 0.00 & 2.0 & 29 \\
\hline Permeate (24 hrs.) & 0.00 & - & 24 \\
\hline Permeate (48 hrs.) & 0.34 & 1.0 & 21 \\
\hline \multicolumn{4}{|c|}{ With Air Scour/BW/Relaxation } \\
\hline Reactor (initial) & 30.93 & 23.00 & - \\
\hline Reactor (24 hrs.) & 76.50 & 29.50 & - \\
\hline Reactor (48 hrs.) & 62.97 & 26.50 & - \\
\hline Permeate (Initial) & 0.00 & 3.50 & 29 \\
\hline Permeate (24 hrs.) & 0.00 & 0.00 & - \\
\hline Permeate (48 hrs.) & 0.00 & 6.00 & 29 \\
\hline
\end{tabular}




\subsection{Main Phase Conclusions}

The effect of filtration flux and the three physical cleaning operations of backwashing, relaxation and air scouring on fouling were studied using a $2^{4}$ factorial design of experiments. Main and interaction effects were identified and assessed using a 4-way ANOVA model as well as linear regression. Filtration flux was the most influential factor effecting membrane fouling. With regards to the cleaning operations, air flow rate and relaxation duration had a larger positive influence on fouling reduction than backwash duration. The interaction effects along with interaction plots provided good insight on two way relationships between main effects such as the antagonistic effect of filtration flux on the three cleaning operations and the synergistic effect of each cleaning operations on filtration flux. The interpretations of three-way interactions using interaction plots identified the adequacy of a short backwash duration for fouling reduction regardless of filtration flux and the difference in behavior of cleaning operation regimes at the two filtration fluxes.

Ideal treatment combinations for fouling reduction while taking into account interpretations from the ANOVA parameters and interaction plots were identified using TMP curves. The treatment combination involving a high air flow rate (15 LPM), long backwash duration (20 sec.) and short relaxation duration ( $5 \mathrm{~min}$.) was identified as the best combination for fouling reduction at the high filtration flux (38 LMH) while short relaxation $(5 \mathrm{~min})$ and backwash (10 sec.) durations and high air flow (15 LPM) were found to be sufficient for the reduction of fouling at the low filtration flux (19 LMH). 
Linear regression via Sum of Squares of Residuals and stepwise regression ( $\alpha$ of 0.10 and 0.15 to accept and reject effects respectively) identified main effects of filtration flux, air flow rate and relaxation duration along with the two way interactions of filtration flux with air flow rate, relaxation duration and the three-way interaction of filtration flux, air flow rate and relaxation duration as important contributors to fouling reduction. Backwash duration was not as significant a contributor to fouling reduction as air flow rate and relaxation duration and thus was eliminated by stepwise regression.

A fractional $2^{3}$ factorial design of experiments was also performed during the Main Phase to study the effect of cleaning operation exclusion on fouling. The evident effect of backwashing on fouling reduction during these experiments suggested the need for the testing of other backwash parameters such as backwash strength and frequency in future fouling studies. These experiments also showed the effectiveness of the combination of all three cleaning parameters compared to air assisted backwashing and air assisted relaxation. Air scouring was found to be the most essential of the three cleaning operations.

Finally, the inclusion of filtration flux as a predictor variable in the $2^{4}$ factorial design of experiment was thought to have limited the outcome of the study. As a result of the different effect of cleaning operations at the two filtration flux levels, a single optimal cleaning regime based on the complete $2^{4}$ factorial design of experiments could not be identified. Overall the full factorial study provided useful insight on the relationships between cleaning operations and their effect on fouling reduction. However, future studies incorporating a factorial design to investigate the effect of operations on fouling reduction should only make use of test variables that reduce fouling. 


\section{CHAPTER 6 - Model Development Phase}

The objective of the Model Development Phase was to develop and evaluate a model for the prediction of fouling over time based on the general linear model (GLM) representing the $2^{4}$ factorial design of experiments performed in the Main Phase Experiments. In Chapter 5, least square estimates of regression coefficients for the general linear model (GLM) representing the $2^{4}$ factorial design of experiments were determined to investigate the importance of main and interaction effects on fouling. Regression coefficients were estimated based on fouling measured at the end of 5 day long experiments. While these regression coefficients were estimated for the complete GLM representing the factorial design, stepwise linear regression identified main and interaction effects that significantly contributed to fouling reduction and thus identified regression coefficients for a reduced GLM that could best represent the results from the $2^{4}$ factorial design. However, while regression coefficients were identified, neither of the two models were developed nor evaluated in Chapter 5.

Since the prediction model was to be based on the Original GLM, the development and evaluation of this original statistical model as well as a number of reduced GLMs were performed during the Model Development Phase. A time basis was then added to the GLM to develop a statistical model for the prediction of fouling over time. The development and evaluation of this prediction model based on the Original GLM are discussed in this chapter. 


\subsection{The Original GLM}

During the Model Development Phase, regression coefficients identified in the Main Phase Experiments were inserted into the complete GLM for the $2^{4}$ factorial design (Equation 3.5 in Chapter 3 Section 3.5.2) resulting in the development of the Original GLM that exactly represented the change in TMP observed during the 16 different experiments (Equation 6.1).

$\Delta T M P_{D a y 5}=-15.8+-9.96 x_{1}+5.55 x_{2}+2.19 x_{3}+4.69 x_{4}+3.34 x_{1} x_{2}+$ $1.65 x_{1} x_{3}+4.81 x_{1} x_{4}+0.0776 x_{2} x_{3}-2.12 x_{2} x_{4}-0.758 x_{3} x_{4}+1.02 x_{1} x_{2} x_{3}-$ $3.56 x_{1} x_{2} x_{4}-1.83 x_{1} x_{3} x_{4}-1.75 x_{2} x_{3} x_{4}-2.48 x_{1} x_{2} x_{3} x_{4}$ (Equation 6.1)

Where,

$x_{1} \rightarrow$ Level $(-1,+1)$ of filtration flux in the treatment combination,

$\mathrm{x}_{2} \rightarrow$ Level $(-1,+1)$ of air flow rate in the treatment combination,

$\mathrm{x}_{3} \rightarrow$ Level $(-1,+1)$ of backwash duration in the treatment combination and

$\mathrm{x}_{4} \rightarrow$ Level $(-1,+1)$ of relaxation duration in the treatment combination.

Regression coefficients representing important main and interaction effects statistically identified by step wise regression were used to develop the reduced GLM (GLM A) shown in Equation 6.2. A second reduced GLM (GLM B) was developed based on the important effects identified by the ANOVA model and interaction plots. GLM B represented a less statistically significant model for evaluation purposes and was developed by excluding regression coefficients representing the insignificant interactions between air flow rate and backwash duration, backwash duration and relaxation duration as well as filtration flux, air flow rate and backwash duration from the Original Model as shown in Equation 6.3. 
$\Delta T M P_{\text {Day5 }}=-15.8+-9.96 x_{1}+5.55 x_{2}+4.69 x_{4}+3.34 x_{1} x_{2}+4.81 x_{1} x_{4}-$

$2.12 x_{2} x_{4}-3.56 x_{1} x_{2} x_{4}$

(Equation 6.2)

$\Delta T M P_{\text {Day } 5}=-15.8+-9.96 x_{1}+5.55 x_{2}+2.19 x_{3}+4.69 x_{4}+3.34 x_{1} x_{2}+$ $1.65 x_{1} x_{3}+4.81 x_{1} x_{4}-2.12 x_{2} x_{4}-3.56 x_{1} x_{2} x_{4}-1.83 x_{1} x_{3} x_{4}-1.75 x_{2} x_{3} x_{4}-$ $2.48 x_{1} x_{2} x_{3} x_{4}$

(Equation 6.3)

Table 6.1 compared the 5 day fouling estimated for each $2^{4}$ factorial experiment using the Original GLM, GLM A and GLM B as well as the Sum of Squares of Residuals (SSR) for each model. Interestingly, GLM A (Equation 6.2) provided a weak representation of the results from the $2^{4}$ factorial experiments as made evident by the high SSR of GLM A compared to the SSR of the Original GLM (Equation 6.1) and GLM B (Equation 6.3). Furthermore, a comparison of the 5 day fouling estimated using GLM A and the Original GLM while taking into account the error associated with the pressure transducers (+/- 5.12 mbar) showed the ineffectiveness of GLM A when estimating the fouling of a number of experiments (most noticeably Experiments 4 and 8). These results indicated that the confidence level $(90 \%)$ used for the identification of important interactions in stepwise regression may have resulted in the exclusion of effects of lower importance but still of some influence to fouling reduction from the reduced model. To ensure that effects contributing to fouling reduction were included in the prediction model thus improving the model's predictability, various subsets of regression coefficients representing lesser important effects that had been originally excluded from GLM A were tested during the development of the prediction model. 
Table 6.1 - Comparison of the fouling predicted by the Original and Reduced GLMs for each experiment and the corresponding Sum of Squares of Residuals.

\begin{tabular}{|c|c|c|c|}
\hline $\begin{array}{c}\text { Experiment } \\
\#\end{array}$ & $\begin{array}{c}\text { TMP (mbar) estimated } \\
\text { by the Original GLM }\end{array}$ & $\begin{array}{c}\text { TMP (mbar) estimated } \\
\text { by the Reduced GLM } \\
\text { A }^{*}\end{array}$ & $\begin{array}{c}\text { TMP (mbar) estimated } \\
\text { by the Reduced GLM } \\
\text { B }^{*}\end{array}$ \\
\hline 1 & -7.65 & -6.52 & -5.96 \\
\hline 2 & -50.9 & -49.8 & -51.3 \\
\hline 3 & -2.76 & -4.96 & -2.94 \\
\hline 4 & -32.5 & -20.7 & -30.6 \\
\hline 5 & -5.38 & -6.52 & -7.07 \\
\hline 6 & -48.7 & -49.8 & -48.4 \\
\hline 7 & -7.17 & -4.96 & -6.99 \\
\hline 8 & -8.96 & -20.7 & -10.8 \\
\hline 9 & -11.4 & -9.62 & -11.3 \\
\hline 10 & -23.9 & -19.5 & -25.7 \\
\hline 11 & -3.72 & -2.31 & -5.42 \\
\hline 12 & -11.2 & -13.0 & -10.8 \\
\hline 13 & -7.79 & -9.62 & -7.97 \\
\hline 14 & -15.1 & -19.5 & -13.2 \\
\hline 15 & -0.896 & -2.31 & 0.799 \\
\hline 16 & -14.9 & -13.0 & -15.3 \\
\hline$S S R$ & 0.000 & 347 & 25.9 \\
\hline
\end{tabular}

*Note: Reduced GLM A - based on results from stepwise regression (elimination of all effects and interactions involving backwash duration); Reduced GLM B - based on ANOVA and interaction plot results (elimination of bottom 3 least significant effects - air flow rate and backwash duration; backwash duration and relaxation duration; filtration flux, air flow rate and relaxation duration). Also, pressure transducer error $=+/-5.12 \mathrm{mbar}$ based on the $0.25 \%$ full scale error associated with the transducer used.

\subsection{Time Based Prediction Model}

The prediction of fouling over time due to the physical cleaning effects of backwashing, air scouring and relaxation was thought to be a beneficial tool for the optimization of these operations employed to reduce fouling. Thus the development of a time based model was sought such that a single unified model would allow for the prediction of fouling by any of the treatment combinations used in the study. 
While, the Original GLM exactly represented the change in TMP observed for all experiments in the $2^{4}$ factorial design, the GLM was found to be limited because the results were day specific i.e. the model only represented the change in TMP on day 5 and thus could only be used to determine the 5 day estimate of fouling due to a treatment combination. Thus it was thought that the change in main and interaction effects (regression coefficients) over time may provide an apt time dependency to the general linear model for fouling prediction over time. To check for the appropriateness of such an approach, TMP changes observed in the first four days of experiments and the corresponding regression coefficients calculated for each of the first four days were used for the prediction of fouling on the $5^{\text {th }}$ day.

Regression coefficients for days 1 to 4 (APPENDIX R) were estimated using the daily change in TMP and least square estimation. Each set of regression coefficients were plotted over time and the curves of best fit (linear, logarithmic and exponential when possible) were obtained to model each coefficient over time. Based on the equations for the trend lines, three Regression Estimation Models (REMs) were used to incorporate time dependency to the complete GLM representing the $2^{4}$ factorial design. Regression Estimation Models 1, 2 and 3 involved the estimation of regression coefficients using the linear, logarithmic and mixed (combination of best fitting curves $\left(R^{2}\right.$ value) for each coefficient) trends of coefficients over time respectively as presented in APPENDIX S.

Regression Estimation Models were inserted into the complete GLM (Equation 3.5 from Chapter 3) resulting in three Preliminary Prediction Models (PPMs) which were evaluated by comparing the Sum of Squares of Residuals of each model with that of the Original GLM $(\operatorname{SSR}=0)$. The percent error of each predicted value was also calculated 
to ensure that while the overall preliminary model represented the Original GLM, the predicted values for each experiment were also a proper representation of the actual values. Table 6.2 shows the Sum of Squares of Residuals as well as the percent error of the predicted TMP values.

Table 6.2 - Comparison of preliminary prediction models for the prediction of fouling after 5 days.

\begin{tabular}{|c|c|c|c|c|c|c|c|}
\hline $\begin{array}{c}\text { Experiment } \\
\#\end{array}$ & $\begin{array}{l}\text { Actual } \\
\text { TMP : } \\
\text { Day } 5\end{array}$ & $\begin{array}{c}\text { TMP } \\
\text { (mbar) - } \\
\text { Proliminary } \\
\text { Prodiction } \\
\text { Model } 1 \\
\end{array}$ & error & $\begin{array}{c}\text { TMP } \\
\text { (mbar) - } \\
\text { Prollminary } \\
\text { Prediction } \\
\text { Model } 2\end{array}$ & $\begin{array}{c}\% \\
\text { error }\end{array}$ & $\begin{array}{c}\text { TMP } \\
\text { (mbar) - } \\
\text { Preliminary } \\
\text { Prodiction } \\
\text { Model } 3 \\
\end{array}$ & $\begin{array}{c}\% \\
\text { error }\end{array}$ \\
\hline 1 & -6.89 & -7.79 & -13.0 & -7.18 & -4.07 & -7.02 & -1.83 \\
\hline 2 & -50.9 & -52.9 & -3.96 & -48.7 & 4.37 & -50.2 & 1.40 \\
\hline 3 & -2.76 & -2.83 & -2.48 & -2.38 & 13.6 & -3.27 & -18.6 \\
\hline 4 & -32.5 & -34.6 & -6.58 & -32.1 & 1.09 & -33.1 & -1.92 \\
\hline 5 & -5.38 & -3.48 & & -3.29 & $y$ & -2.14 & \\
\hline 6 & -48.7 & -47.7 & 2.16 & -43.3 & 11.1 & -45.2 & 7.14 \\
\hline 7 & -7.17 & -7.31 & -1.91 & -6.90 & 3.77 & -7.70 & -7.37 \\
\hline 8 & -8.96 & -7.96 & 11.1 & -7.80 & 13.0 & -7.27 & 18.9 \\
\hline 9 & -11.4 & -11.4 & 0.60 & -11.1 & 3.20 & -10.4 & 8.81 \\
\hline 10 & -23.9 & -27.8 & -16.5 & -26.3 & -10.3 & -25.1 & -5.20 \\
\hline 11 & -3.72 & -2.97 & 20.4 & -2.83 & 24.0 & -2.74 & 26.5 \\
\hline 12 & -11.2 & -14.5 & -29.6 & -13.8 & -23.3 & -12.5 & -11.9 \\
\hline 13 & -7.79 & -5.58 & 28.3 & -4.75 & & -5.13 & \\
\hline 14 & -15.1 & -14.6 & 3.20 & -12.9 & 14.8 & -12.2 & 19.1 \\
\hline 15 & -0.90 & -1.41 & & -1.17 & & -1.15 & -28.1 \\
\hline 16 & -14.9 & -17.5 & -17.6 & -16.1 & -7.80 & -15.3 & -2.75 \\
\hline $\begin{array}{l}\text { Sum of } \\
\text { Squares of } \\
\text { Residuals }\end{array}$ & 0 & 54.4 & & 69.8 & & 47.9 & \\
\hline
\end{tabular}

Highlighted values identify the noticeably large \% error $(>30 \%)$ associated with fouling predicted by a preliminary prediction model. 
In terms of the goodness of fit to the Original GLM, PPM3 had the lowest SSR while PPM1 best represented the actual TMP values for each experiment. However, in all three sets of predicted values, a number of experiments were found to be poorly represented by the Preliminary Prediction Models. For instance, in the set of values predicted using PPM1, a 58\% overestimation was noticed for Experiment 15 while a $60 \%$ underestimation of fouling was obtained for Experiment 5 when using PPM3. Importantly, the lower fouling experiments had the largest error associated when any of the three models were used, while the higher fouling experiments had a better model fit.

While high errors would generally bring about concerns regarding the efficiency of a prediction model, the high errors for the low fouling experiments were attributed to the potential error ( $+/-5.12 \mathrm{mbar})$ associated with the pressure transducers used in the study. As such, all three PPMs provided reasonable estimations of fouling for all experiments. The comparability of the low TMP values of low fouling experiments to the error associated with the pressure transducers suggested that a high accuracy for the prediction of low fouling experiments by a model cannot be expected from the developed model. This limitation of the Preliminary Prediction Models when predicting lower levels of fouling was thus identified as a potential weakness of the model since the goal of the factorial design was to identify cleaning regimes minimizing fouling.

The objective of the Model Development Phase was to identify a single unified model for the prediction of fouling over time. However, a clear cut identification of the best of the three PPMs for the prediction of fouling over time was not evident at this stage of the Model Development Phase. Furthermore, all three PPMs included effects and interactions of little or no significance to fouling reduction which are usually excluded 
from GLMs. As such, it was thought that the simplification of the PPMs by excluding insignificant effects and interactions may improve the predictability of the model and help identify the most ideal model for the prediction of fouling over time.

Table 6.3 shows the important main and interaction effects (regression coefficients) identified by the ANOVA model/interaction plots, general linear modelling via Sum of Squares of Residuals comparison and stepwise regression and even the graphical means of identifying important interactions in the Half Normal Plot (APPENDIX T). From Table 6.3 it can be seen that different methods of identification resulted in different predictions of which parameters were most important.

Table 6.3 - Summary of important effects (regression coefficients) identified in the study.

\begin{tabular}{|c|c|}
\hline Analysis Methods & Regression Coefficients* \\
\hline ANOVAVinteraction plots & $\boldsymbol{\beta}_{1}, \boldsymbol{\beta}_{2}, \boldsymbol{\beta}_{3}, \boldsymbol{\beta}_{4}, \boldsymbol{\beta}_{12}, \boldsymbol{\beta}_{13}, \boldsymbol{\beta}_{14}, \boldsymbol{\beta}_{24}, \boldsymbol{\beta}_{124}, \boldsymbol{\beta}_{134}, \boldsymbol{\beta}_{234}$, \\
\hline Half Normal Plot & $\boldsymbol{\beta}_{123}, \boldsymbol{\beta}_{2}, \boldsymbol{\beta}_{4}, \boldsymbol{\beta}_{12}, \boldsymbol{\beta}_{13}, \boldsymbol{\beta}_{14}, \boldsymbol{\beta}_{234}$ \\
\hline GLM (SSR and Stepwise regression) & $\boldsymbol{\beta}_{1}, \boldsymbol{\beta}_{2}, \boldsymbol{\beta}_{4}, \boldsymbol{\beta}_{12}, \boldsymbol{\beta}_{14}, \boldsymbol{\beta}_{24}, \boldsymbol{\beta}_{124}$ \\
\hline
\end{tabular}

* Variables defined in Table 5.1 in Chapter 5

To ensure that effects contributing to the reduction of membrane fouling were included in the prediction model an initial simplified model was developed that included all the coefficients representing important effects identified by the ANOVA model and interaction plots in Table 6.3. A whole series off simplified models was then developed incorporating a stepwise elimination of individual and groups of coefficients till a simplified model representing the effects identified using the stepwise regression was achieved. These resulting simplified versions of the preliminary models were called the Simplified Prediction Models (SPMs). 
Though the Preliminary Prediction Models were limited when representing the TMP of low fouling experiments, the experiments with the highest and lowest fouling (Experiments 2 and 15 respectively) were used as test experiments during the identification process of the SPM. Taking into account the need for better predictability of lower fouling experiments as well as the need for a single model to predict fouling at both fluxes, the initial SPM that included all the important effects determined by the interaction plots was identified as the best reduced model for the prediction of both high and low fouling experiments. This identified SPM also took into account the use of the three Regression Estimation Models used to provide the time basis to the prediction model. Table 6.4 shows the predicted values determined for Experiments 2 and 15 by the simplified model using all three REMs and also includes the SSR of each Simplified Prediction Model.

Table 6.4 - Comparison of TMP prediction by the Simplified Prediction Model (SPM) when using three different Regression Estimation Models (REM).

\begin{tabular}{|c|c|c|c|c|c|c|c|}
\cline { 2 - 7 } & \multicolumn{3}{c|}{$\begin{array}{c}\text { Experiment 15 } \\
\text { (Lowest fouling) }\end{array}$} & \multicolumn{3}{c|}{$\begin{array}{c}\text { Experiment 2 } \\
\text { (Highest fouling) }\end{array}$} \\
\cline { 2 - 8 } & $\begin{array}{c}\text { SPM 1 } \\
\text { (REM 1) }\end{array}$ & $\begin{array}{c}\text { SPM 2 } \\
\text { (REM 2) }\end{array}$ & $\begin{array}{c}\text { SPM 3 } \\
\text { (REM 3) }\end{array}$ & $\begin{array}{c}\text { SPM 1 } \\
\text { (REM 1) }\end{array}$ & $\begin{array}{c}\text { SPM 2 } \\
\text { (REM 2) }\end{array}$ & $\begin{array}{c}\text { SPM 3 } \\
\text { (REM 3) }\end{array}$ \\
\hline \multirow{2}{*}{$\begin{array}{c}\text { Day } \\
\mathbf{5}\end{array}$} & \begin{tabular}{c} 
Actual (mbar) \\
\cline { 2 - 8 }
\end{tabular} & -0.896 & -0.896 & -0.896 & -50.9 & -50.9 & -50.9 \\
\cline { 2 - 8 } & $\begin{array}{c}\text { Predicted } \\
\text { (mbar) }\end{array}$ & 1.11 & 1.08 & 1.37 & -52.7 & -48.5 & -50.0 \\
\cline { 2 - 8 } & $\begin{array}{l}\text { Error (\%) } \\
\text { Prediminary } \\
\text { Modiction } \\
\text { (\%) }\end{array}$ & -24 & -21 & -53 & -3.5 & 4.8 & 1.9 \\
\hline $\begin{array}{c}\text { Sum of Squares of } \\
\text { Residuals for all } \\
\text { experiments }\end{array}$ & 74 & 88 & 68 & 74 & 88 & 68 \\
\hline
\end{tabular}

Note: SPM includes most parameters of the Original GLM except the interactions between air flow rate and backwash duration, backwash duration and relaxation duration and also filtration flux, air flow rate and relaxation duration. 
While the SPM 3 resulted in the closest model resemblance to the Original GLM, the fouling predicted for the lowest fouling experiment resulted in an error increase from $28 \%$ (error of PPM 3) to $53 \%$ (error of SPM 3). Since it was expected that the error would decrease due to the simplification of the model by excluding least influential effects and interactions, the increased inefficiency of SPM 3 suggested that this regression estimation model was not suitable for use in the prediction model. While considerable decreases in the error of the predicted fouling for the low fouling experiment was noticed when using SPM 1 (58\% error of PPM 1 to $24 \%$ error of SPM 1) and SPM 2 (31\% error of PPM 2 to $21 \%$ error of SPM 2), neither of the two models could be identified as the best model for fouling prediction over time. Also, the lack of change in the fairly efficient ( $<5 \%$ error) prediction of the high fouling experiment (seen in Table 6.4) did not provide further insight on the better of the two models.

While a single model was not yet identifiable, the simplification of the models did show the difference of physical cleaning behavior based on the level of fouling. Main Phase Experiments showed that the lowest and highest fouling experiments tested were influenced by the levels of filtration flux. As such, the different effects of model simplification on the improvement of the model prediction at the two fouling levels confirmed the difference in contributions of effects of cleaning operations at the two filtration fluxes. Due to the ability of the model to predict the TMP of high flux experiments, it was decided to further evaluate the model over a longer experimental run to identify the better of the two regression estimation approaches (REM 1 or 2) as well as further evaluate the predictability of the model over time. For the longer fouling study the regression coefficients from Day 5 were also incorporated into the Regression Estimation 
Models to increase the robustness of the estimation of regression coefficients and in hopes that the better estimation of the coefficients would result in lower prediction error of the lower fouling experiments. The regenerated models for the regression coefficients can be seen in Table 6.5.

Table 6.5 - Regenerated equations for the estimation of regression coefficients over time by Option 1 and 2

\begin{tabular}{|c|c|c|c|c|}
\hline \multirow{2}{*}{$\begin{array}{l}\text { Regression } \\
\text { Coefficient }\end{array}$} & \multicolumn{2}{|c|}{$\begin{array}{c}\text { Regression Estimation } \\
\text { Model } 1\end{array}$} & \multicolumn{2}{|c|}{$\begin{array}{c}\text { Regression Estimation Model } \\
2\end{array}$} \\
\hline & Linear & $\mathbf{R}^{2}$ & Logarithmic & $\mathbf{R}^{2}$ \\
\hline $\boldsymbol{\beta}_{\mathbf{0}}$ & $-1.5851 x-8.0679$ & 0.986 & $-3.941 \ln (x)-9.0502$ & 0.984 \\
\hline $\boldsymbol{\beta}_{1}$ & $-0.9719 x-5.4854$ & 0.934 & $-2.493 \ln (x)-6.0147$ & 0.993 \\
\hline $\boldsymbol{\beta}_{2}$ & $0.7268 x+1.748$ & 0.982 & $1.72455 \ln (x)+2.2658$ & 0.921 \\
\hline $\boldsymbol{\beta}_{3}$ & $-0.0088 x+2.5004$ & 0.001 & $0.1613 \ln (x)+2.3724$ & 0.052 \\
\hline$\beta_{4}$ & $0.5277 x+1.8989$ & 0.970 & $1.2262 \ln (x)+2.3078$ & 0.846 \\
\hline$\beta$-hat ${ }_{12}$ & $0.4867 x+0.9332$ & 0.993 & $1.1872 \ln (x)+1.2566$ & 0.955 \\
\hline$\beta$-hat 13 & $0.092 x+1.4003$ & 0.177 & $0.3235 \ln (x)+1.3537$ & 0.354 \\
\hline B-hat 14 & $0.5169 x+2.0165$ & 0.876 & $1.1824 \ln (x)+2.435$ & 0.740 \\
\hline${ }^{*} \beta-$ hat $_{23}$ & $0.2385 x-1.3421$ & 0.662 & $0.5071 \ln (x)-1.1121$ & 0.483 \\
\hline B-hat 24 & $-0.3157 x-0.5949$ & 0.981 & $-0.789 \ln (x)-0.786$ & 0.991 \\
\hline${ }^{*} \beta-$ hat $_{34}$ & $-0.3243 x+0.8099$ & 0810 & $-0.799 \ln (x)+0.6025$ & 0.795 \\
\hline${ }^{\star} \beta-$ hat $_{123}$ & $0.1028 x+0.5518$ & 0.477 & $0.2398 \ln (x)+0.6305$ & 0.420 \\
\hline$\beta$-hat 124 & $-0.4281 x-1.4684$ & 0.964 & $-1.05 \ln (x)-1.7478$ & 0.936 \\
\hline$\beta$-hat ${ }_{134}$ & $-0.2032 x-0.8177$ & 0.675 & $-0.513 \ln (x)-0.9357$ & 0.696 \\
\hline$\beta$-hat 234 & $-0.1506 x-1.1219$ & 0.691 & $-0.422 \ln (x)-1.1698$ & 0.876 \\
\hline$\beta$-hat 1234 & $-0.2553 x-1.2654$ & 0.921 & $-0.624 \ln (x)-1.4343$ & 0.887 \\
\hline
\end{tabular}

Where $x=$ Time (days); "Lighter text denotes regression coefficients excluded from the SPM.

\subsection{Simplified Prediction Model Evaluation}

While a number of 10 day long experimental runs based on the Main Phase Experiments were initially planned to help evaluate the Simplified Prediction Model, 
only a single 10 day long run was achieved due to apparatus malfunctions and time restriction of this research project. The single experiment however was useful for a simple analysis of the SPM for the prediction fouling over time. The SPM using both Regression Estimation Models developed in Section 6.1 were used to predict fouling over time for a 10 day experimental run involving air scouring (15 LPM), relaxation duration (15 min.), backwash duration (20 sec.) and filtration flux (38 LMH). Figure 6.1 compares the predicted TMP over time using the two SPMs and the actual TMP observed during the experimental run.

Comparing the prediction of SPM using the Regression Estimation Model 1 (linear estimation of regression coefficients) with the actual 10 day change in TMP showed that the SPM provided a $20 \%$ over prediction of fouling from the actual 10 day TMP of -20.34 mbar. In addition, the predictions of the SPM did not represent the fouling trend over time and thus Regression Estimation Model 1 was not considered suitable. In the case of the SPM using Regression Estimation Model 2 (logarithmic estimation of regression coefficients), an $11 \%$ underestimation of TMP was predicted. Taking into account the expected traducer error $(+/-0.25 \%$ full scale) and the ability of the model to provide a similar trend of fouling over time as the actual change in TMP of the 10 day experiment, the SPM using Regression Estimation Model 2 (logarithmic estimation of coefficients) was identified as the ideal model for the prediction of fouling over time in this study.

As previously mentioned, the reduced model best representing the prediction of fouling at the high and low fouling levels was chosen as the SPM for the prediction of fouling over time. However due to differences in effects of physical cleaning operations 
at the two fluxes, the unified SPM may have included or eliminated regression coefficients specific to high flux conditions thus resulting in poor prediction of fouling.

Figure 6.1 - Evaluation of Simplified Prediction Models for the prediction of fouling over 10 days (Flux $\rightarrow 38$ LMH).

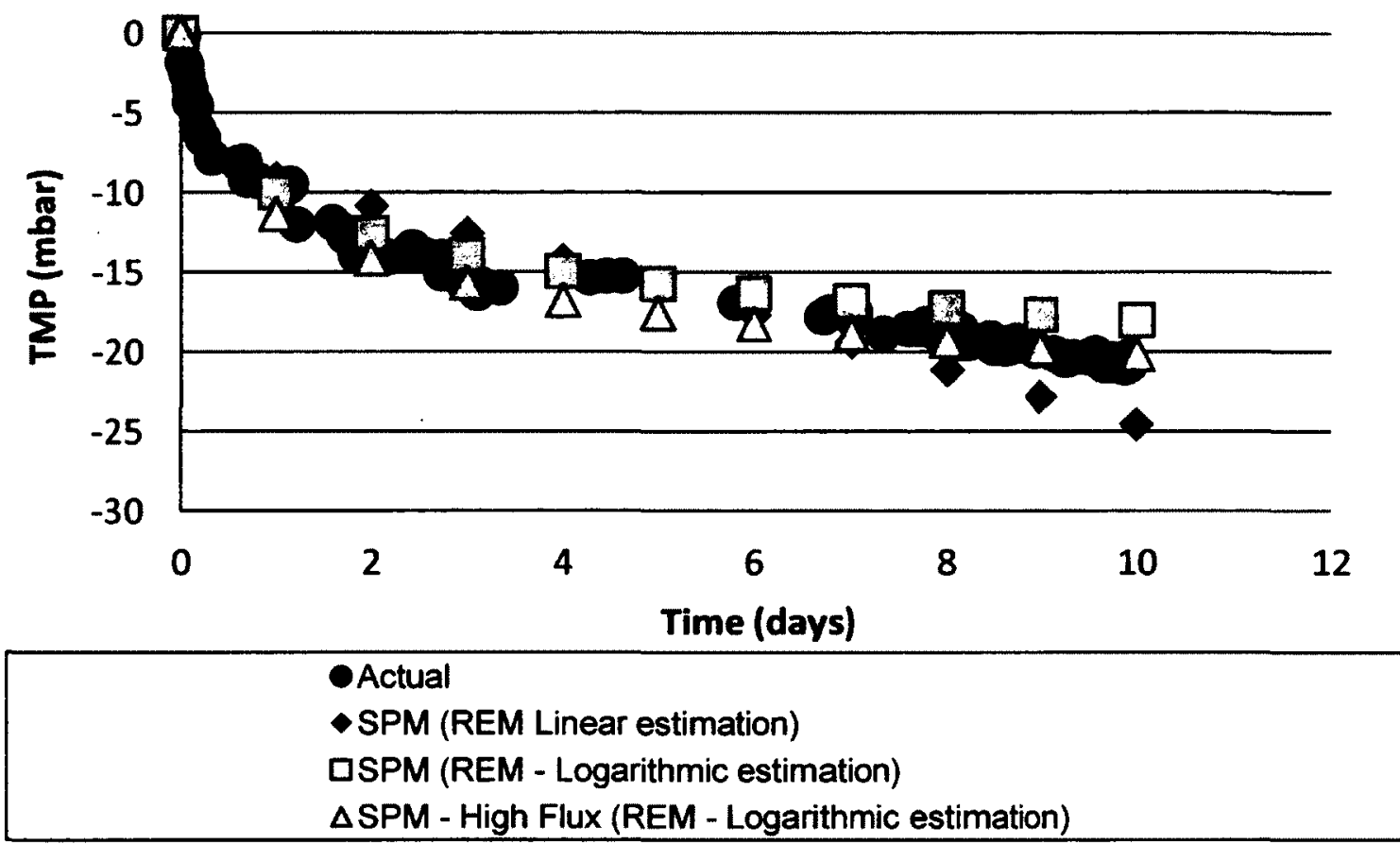

Since the 10 day long evaluation run involved a high filtration flux (38 LMH), the results from the two Main Phase Experiments with the most fouling at this flux level (Experiments 2 and 6) were used to identify a flux specific SPM. The SPM involving the exclusion of the regression coefficients $\beta_{13}, \beta_{24}, \beta_{34}, \beta_{123}$ was found to be the best represent the prediction of the 5 day fouling within $10 \%$ of the actual TMP values for these experiments. This SPM using Regression Estimation Model 2 (i.e. SPM-High Flux) was used to predict the 10 day fouling as shown in Figure 6.1. The SPM-High Flux model using a logarithmic estimation of regressions coefficients was efficient ( $1 \%$ error) at predicting the TMP at the end of the 10 day experiment. However, no conclusions 
could be made on the efficiency of the SPM-High Flux model with just a single experimental run. While a similar analysis for low flux conditions was intended, an insufficient amount of time for experiment completion was available. In addition, the model development itself seemed somewhat cumbersome and lacking in practical value.

\subsection{Conclusions}

The development of a unified model for the long term prediction of fouling of all $2^{4}$ factorial experiments employed in the Main Phase Experiments was attempted during the Model Development Phase. The general linear model (GLM) representing the $2^{4}$ factorial design of experiments was used as the base model for the development of the prediction model. Based on daily fouling data from the first 4 days of 5 day long Main Phase Experiments, linear, logarithmic and mixed estimates of regressions coefficients for the GLM were determined to provide a time basis to the GLM. Thus a Preliminary Prediction Model was developed using each method of regression coefficient estimation and used to determine the 5 day fouling of each $2^{4}$ factorial experiment performed. A comparison of the predictions from the Preliminary Prediction Models with those of the Original GLM showed that the Preliminary Prediction Models were limited in estimating fouling under less fouling conditions which was an important practical aspect to the model.

A Simplified Prediction Model (SPM) was developed by reducing the Preliminary Prediction Models to improve the efficiency of the prediction model. The prediction capabilities of the SPM model were evaluated using a 10 day long fouling experiment. 
Results from the evaluation showed that the logarithmic estimation of regression coefficients was best suited for the SPM. The evaluation of the SPM showed that the model was able to represent the fouling trend over time while providing reasonable predictions of fouling over the evaluation period. A flux specific SPM - High Flux model was also developed during this study for the prediction of fouling for high flux/ high fouling operations which seemed efficient for the prediction of high fouling. However, insufficient evaluation of the model was performed to make a conclusion on the effectiveness of the high flux specific model.

While the unified SPM - REM 2 model was found to be efficient for the prediction of fouling over time in this study, the model was found to be limited in its prediction capabilities specifically for low fouling experiments. Due to the extremely low TMP associated with low fouling experiments, a high accuracy of the prediction of low fouling experiments cannot be expected. As such, the practicality of using such a model for the prediction of fouling of experiments aimed at reducing fouling is hindered. With regards to the flux specific SPMs, though the SPM - High Flux model seemed to be able to predict fouling of higher fouling experiments, the efficiency of the SPM - Low Flux Model is not known. In both cases, further evaluation of each model is required using numerous 10 day long experiments before any final conclusion on the use of the factorial design to develop a fouling prediction model can be made. 


\section{CHAPTER 7 - Final Conclusions}

The growing popularity of membranes in drinking water treatment has brought about the need for a better understanding of processes used to reduce the fouling of membranes. While physical cleaning processes like air scouring, backwashing and relaxation are common operations in wastewater treatment, literature on the effect of these processes especially relaxation for fouling attenuation in drinking water treatment is lacking. Thus, an investigation on the combined effect of the physical cleaning operations air scouring, backwashing and relaxation for the reduction of membrane fouling in drinking water treatment was carried out in this research study. A bench scale apparatus incorporating a ZW-1 hollow fiber PVDF ultrafiltration membrane from GE Zenon was used for the research. The study was carried out in three phases, the Preliminary Study Phase, the Main Phase Experiments and the Model Development Phase. The Preliminary Study Phase and Main Phase Experiments made use of factorial design of experiments for the purpose of analysing the three cleaning operations on membrane fouling. A general linear model based on the factorial study carried out in the Main Phase Experiments was used as the basis for the development of a fouling prediction model in the Model Development Phase. 


\subsection{Preliminary Phase Conclusions}

The effect of relaxation parameters and flow operations were analysed in the Preliminary Study Phase. A $2^{2}$ factorial design of experiments investigating the effect of relaxation duration ( 5 and $15 \mathrm{~min}$.) and relaxation interval ( 2 and $4 \mathrm{hr}$.) found that fouling was minimized when using short $(5 \mathrm{~min}$.) and less frequent $(4 \mathrm{hr}$.) relaxation for dead-end flow operations while more frequent relaxation intervals $(2 \mathrm{hr}$.$) with either relaxation$ duration minimized fouling for continuous flow operations. Overall incorporation of relaxation along with periodic backwashing and continuous air scouring was found to be effective for the reduction of fouling during the Preliminary Study Phase.

Dead-end flow operations resulted in a high rate of membrane fouling due to the accumulation of foulants in the reactor tank while continuous flow operation which reached a steady state water quality in the reactor tank fouled at a slower rate. Thus continuous flow operations with $\mathbf{8 0 \%}$ recovery were found to be ideal for experiments investigating the effect of physical cleaning operations on membrane fouling during 5 day long Main Phase Experiments.

\subsection{Main Phase Conclusions}

Main phase experiments made use of a $2^{4}$ factorial design of experiments for the purpose of analysing the effect of air scouring flow rate, backwash duration, relaxation duration as well as filtration flux on membrane fouling. The main and interaction effects of the four operations were determined using two approaches: the ANOVA approach and the regression approach, both of which provided identical results. While main and two- 
way interactions were interpretable by direct examination of the effects, interaction plots were required to confirm interpretations of two-way interactions as well as help interpret higher order three-way interactions. These interaction plots generated using the ANOVA model were useful in identifying synergistic or antagonistic relationships between the four operations.

2-way interaction plots for the overall main phase $2^{4}$ factorial experiments showed that filtration flux had an antagonistic relationship with all three physical cleaning operations while each cleaning operation had a synergistic relationship with filtration flux. The importance of high air flow rate for fouling reduction was also shown in two-way interaction plots. Three way interactions showed the differences in the effect of cleaning operations at the two filtration fluxes such as the need for long backwash (20 sec.) or relaxation duration (15 min.) at the high flux while short backwash $(10 \mathrm{sec}$.) and relaxation durations ( $5 \mathrm{~min}$.) were found to be sufficient at the low filtration flux.

While interaction plots were helpful in identifying various combinations of cleaning operation parameters ideal for the reduction of fouling, the plots were unable to provide a single best combination of cleaning operations for fouling reduction based on the factorial study. However, the interpretation of the various relationships from interaction plots was helpful during the identification of optimal combinations for the reduction of fouling at the two filtration fluxes using TMP curves.

A cleaning regime involving short backwash and relaxation durations assisted by high air scour flow rate was identified as the best regime for fouling reduction at the low filtration flux. In the case of the high filtration flux, the best cleaning regime involved the 
use of long backwash and short relaxation durations assisted by high air flow rate. Both regimes were thought to best optimize the use of the physical cleaning parameters while minimizing losses in permeate production.

Interaction plots were unable to provide a comprehensive determination of all important effects and interactions that contributed to fouling reduction during the study. While linear regression via the comparison of the Sum of Squares of Residuals was able to identify important effects and interactions, stepwise linear regression $(\alpha=0.10$ for acceptance, $\alpha=0.15$ for rejection) was required to statistically verify the identified important reactions. With regards to the unimportant interactions, all effects and interactions involving backwash duration were identified as unimportant using step wise linear regression signifying the lack of contribution of backwash duration to fouling reduction.

A fractional $2^{3}$ factorial design of experiments was also performed under the Main Phase Study. This design of experiments excluded individual physical cleaning operations from the typical regime used in the study to compare the fouling reducing efficiency of the combination of the three physical cleaning operations with cleaning regimes only involving two of the three operations. Results from these experiments showed that though backwash duration was found to have no significant effect on fouling reduction during the $2^{4}$ factorial study, the operation of backwashing itself had a major effect in reducing fouling. Furthermore, the use of the three physical cleaning operations combined was found to be effective in reducing fouling compared to a regime using only two cleaning options. Further evaluation of the various sequences of air assisted backwashing and relaxation while using intermittent air flow rate might prove useful for 
the optimization of the regime while being cost effective and minimizing loss of productivity. The combination of the three cleaning operations in a physical cleaning regime can be promoted as a viable means of fouling reduction compared to common procedure that either use air assisted backwashing or air assisted relaxation.

Finally, while optimal cleaning regimes were identified based on the results of the factorial design, the use of filtration flux and backwash duration as predictor variables in the factorial design was found to limit the overall outcome of the fouling study. In summary, the key findings from the Main Phase Experiments were as follows:

- Interaction plots are helpful in providing an understanding of the relationship between effects but neither effective in identifying the best cleaning regime for the reduction of fouling nor the identification of important effects and interactions.

- Linear regression using a Sum of Squares of Residuals was useful for the identification of important effects and interaction however stepwise linear regression is required for statistical verification of the identified important effects and interactions.

- Under low filtration flux conditions, short backwash and relaxation durations assisted by high air flow rate is ideal for fouling reduction at the water quality parameters tested.

- Under high filtration flux conditions, long backwash and short relaxation durations assisted by high air flow rate is ideal for fouling reduction at the water quality parameters tested.

- Relaxation duration and air flow rate contribute to fouling reduction while backwash duration does not.

- The operation of backwashing is essential for fouling reduction even though backwash duration does not contribute to this cause.

- The combination of all three cleaning operations is more efficient in reducing fouling that air assisted backwashing or air assisted relaxation. 


\subsection{Model Development Phase}

In addition to the use of the statistical model to help assess the importance of the cleaning regimes, another component of the research project was the determination of a prediction model for fouling over time. The general linear model (GLM) representing the $2^{4}$ factorial design in the Main Phase Experiments was used as the base model for the development of the prediction model. A time basis was added to the GLM by including the logarithmic estimation of regression coefficients over time based on the daily changes in TMP during the 5 day Main Phase Experiments. The modified GLM was then reduced based on subsets of effects and interactions eliminated by linear regression. A Simplified Prediction Model was thus developed for the prediction of fouling at both the high and the low flux levels. While this unified model was effective in predicting fouling over time for a high fouling experiment, the effectiveness of the model to predict fouling of low fouling experiments was not tested and thus the model requires further evaluation. Furthermore, the low TMP associated with low fouling experiments comparable to the error of the pressure transducers used in this study was thought to hinder the accuracy of low fouling predictions.

Flux based prediction models were also developed during this phase. Due to time restrictions for project completion only a single 10 day experiment was run to evaluate the efficiency of the model at the higher flux. Fouling at the end of the 10 day experiment was measured as -20.34 mbar while the model predicted a TMP of -20.13 mbar resulting in an error of $1 \%$. While, the prediction model was able to predict the fouling at the end of the 10 day experiment and represent the fouling trend over time, a conclusion on the efficiency of the model could not be made based on a single run. It is also unknown if the 
model developed for the low flux is effective in predicting fouling at the low flux since the model was not evaluated.

\subsection{Future Studies}

The results from this study open the door to a large avenue of future studies that can be performed. The experiments only examined the effects of cleaning operation on fouling by a specific type of water. Thus experiments examining the effect of water quality on the operational regimes are required in more detail. These would help validate that the statistical method employed along with the linear regression approach used in the study. Examination of water quality would also help ascertain which physical cleaning methods respond best to the different types of fouling that may occur in a membrane system. Since backwash duration was found to have an insignificant effect on fouling reduction, backwash parameters such as backwash frequency and backwash strength (i.e. backwash flux) need to be further evaluated while a more detailed analysis of backwash duration may also be insightful. These parameters along with air scouring intervals for intermittent scouring could be evaluated with the approach taken. An evaluation of various sequences of air scouring, backwashing and relaxation to optimize the cleaning operation combination would also be beneficial. In addition, only one test was run to confirm the time based model. Additional long term tests of fouling using the operational regimes from the Main Phase Experiments are required to further evaluate the unified as well as flux based Simplified Prediction Models developed in this study. 


\section{REFERENCES}

Aoustin, E., Schafer, A.I., Fane, A.G., Waite, T.D., 2001. Ultrafiltration of natural organic matter. Separation and Purification Technology 22-23, 63-78.

Amy, G., 2008. Fundamental understanding of organic matter fouling of membranes. Desalination 231, 44-51.

Anselme, C. Mandra, V., Baudin, I., Mallevialle, J., 1994. Optimum use of membrane processes in drinking-water treatment. Water Supply. Vol. 12 No. 1-2, SS2-1 - SS2-11.

Arnal, J.M., Garcia-Fayos, B., Verdu, G., Lora, J., 2009. Ultrafiltration as an alternative membrane technology to obtain safe drinking water from surface water: 10 years of experience on the scope of the AQUAPOT project. Desalination 248, 34-41.

AWWA, 2005. Microfiltration and Ultrafiltration Membranes for Drinking Water Manual of Water Supply Practices, M53 (1st Edition). American Water Works Association (AWWA), 2005. Retrieved from: http://www.knovel.com.proxy.library.carleton.ca/web/portal/basic_search/display?_EXT KNOVEL DISPLAY bookid $=3639$

BBC Research 2011. Advanced technologies for municipal water treatment - Report Highlights, Report Code: MSR036C Published April 2011. Retrieved from: http://www.bccresearch.com/report/advanced-tech-municipal-water-treatment$\underline{\text { mst036c.html }}$

Bottino, A., Capannelli, G., Comite, A., Mangano, R., 2009. Critical flux in submerged membrane bioreactors for municipal wastewater treatment. Desalination 245, 748-753.

Braak, E., Alliet, M., Schetrite, S., Albasi, C., 2011. Aeration and hydrodynamics in submerged membrane bioreactors. Journal of Membrane Science 379, 1-18.

Cabassud, C., Laborie, S., Durand-Bourlier, L., Laine, J.M., 2001. Air sparging in Ultra filtration: relationship between flux enhancement, cake characterization. Journal of Membrane Science 181, 57-69.

Choi, H., Kim, H.-S., Yeom, I.T., Dionysiou, D.D., 2005. Pilot plant study of an ultrafiltration membrane system for drinking water treatment operated in the feed and bleed mode. Desalination 172, 281-291.

Chen, Y., Dong, B.Z., Gao, N.Y., Fan, J.C., 2007. Effect of coagulation pretreatment on fouling of an ultrafiltration membrane. Desalination 204, 181-188. 
Costa, A.R., de Pinho, M.N., 2006. Comparison of the performance of UF and NF in surface water treatment. Desalination 199, 73-75.

Cui, Z.F., Chang, S., Fane, A.G., 2003. The use of gas bubbling to enhance membrane processes (review). Journal of membrane Science 221, 1-35.

Daigger, G.T., Lozier, J.C., Crawford, G.V. 2006. Water reuse applications using membrane technology. Water Environment Foundation.

Domany, Z., Galambos, I., Vatai, G., Bekassy-Molnar, E., 2002. Humic substance removal from drinking water by membrane filtration. Desalination 145, 333-337

D'Souza, N.M., Mawson, A.J., 2005, Membrane Cleaning in the Dairy Industry: A review. Critical Reviews in Food Science and Nutrition 45, 125-134.

Gao, W., Liang, H., Ma, J., Han, M., Chen, Z., Han, Z., Li, G., 2011. Membrane fouling control in ultrafiltration technology for drinking water production: A review. Desalination 272, 1-8.

Green, J.H., Tylla, M., 1998. A comparison of ultrafiltration on various river waters. Desalination 119, 79-84.

Guo, X., Li, Q., Hu, W., Gao, W., Liu, D., 2008. Ultrafiltration of dissolver organic matter in surface water by a polyvinylchloride hollow fiber membrane. Journal of Membrane Science 327, 254-263.

Guo, X., Gao, W., Li, J., Hu, W., 2009. Fouling and cleaning characteristics of ultrafiltration of hydrophobic dissolved organic matter by a polyvinyl chloride hollow fiber membrane. Water Environment Research Vol:81 Number 6, 626-632.

Hagen, K., 1998. Removal of particles, bacteria and parasites with ultrfiltration for drinking water treatment. Desalination 119, 85-91.

Hendricks, D., 2011. Fundamentals of water treatment unit processes - Physical, chemicals and biological. CRC Press, Taylor and Francis Group LLC, 2011.

Hilal, N., Ogunbiyi, O.O., Miles, N.J., Nigmatullin, R., 2005. Methods employed for control of fouling in MF and UF membranes: A Comprehensive Review. Separation Science and Technology 40 1957-2005.

Hong, S., Krishna, P., Hobbs, C., Kim, D., Cho, J., 2005. Variations in BW efficiency during colloidal filtration of hollow fiber microfiltration membranes. Desalination 173, $257-268$.

Hwang, K-J., Chan, C-S., Tung, K-L. 2009. Effect of Backwash on the performance of submerged membrane filtration. Journal of Membrane Science 330, 349-356 
Jacangelo, J. G., Madec, A., Schwab, K. J., Huffman, D.E., Mysore, C.S., 2005. Advances in the use of low-pressure, hollow fiber membranes for the disinfection of water. Water Supply, Vol 5 No 5, pp 109-115.

Jiang, T., Kennedy, M.D., van der Meer, W.G.J. 2003. The role of blocking and cake filtration in MBR fouling. Desalination 157, 335-343.

Jiang, T., Kennedy, M.D., Guinbourg, B.F., Vanrollegham, P. A., Schippers, J.C. 2005. Optimising the operation of a MBR pilot plant by quiantitative analysis of the membrane fouling mechanism. Water Science and Technology Vol. 51 No 6-7 pp 19-25

Judd, S., 2002. Submerged membrane bioreactors: flat plate or hollow fiber? Filtration \& Separation, 2002. Vol. 39, Issue: 5, 30-31.

Judd, S., Judd, C., 2011. The MBR Book - Principles and Applications of Membrane Bioreactors in Water and Wastewater Treatment (2nd Edition). Elsevier Ltd, 2011.

Kabsch-Korbutowicz. M., Bilyk. A., Molczan. M., 2006. The effect of feed water pretreatment on ultrafiltration membrane performance. Polish Journal of Environmental Studies Vol 15, No. 5, 719-725.

Katsoufidou, K., Yiantsios, S.G., Karabeles, A.J., 2008. An experimental study of UF membrane fouling by humic acid and sodium alginate solutions: the effect of backwashing on flux recovery. Desalination 220, 214-227.

Konieczny, K., 1998. Disinfection of surface and ground waters with polymeric ultrafiltration membranes. Desalination 119, 251-258.

Kutner, M.H., Nachtsheim, C.J., Neter, J., Li., W., 2005. Applied linear statistical models (5th Edition). The McGraw Hill Companies Inc., 2005

Laine, J.M., Vial, D., Moulart, P., 2000. Status after 10 years of operation - overview of UF technology today. Desalination 131 (2000) $17-25$.

Le Clech, P., Jefferson, B., Chang, I.S., Judd, S.J., 2003. Critical Flux determination by the flux-step method in a submerged membrane bioreactor. Journal of Membrane Science Vol: 227, Iss:1-2, 81-93

Li, X., Chu, H.P., 2003. Membrane bioreactor for the drinking water treatment of polluted surface water supplies. Water Research 37, 4781-4791

Li, S., Heijman, S.G.J., Verberk, J.Q.J.C, Verliefde, A.R.D., Kemperman, A.J.B., van Dijk, Amy, G., 2009. Impact of backwash composition on ultrafiltration fouling control. Journal of Membrane Science 344, 17-25. 
Li, Y., Zhang, W., Zhang, X., Chen, C., Wang, J., 2010. Characterization of fouling in immersed polyvinylidene hollow fiber membrane ultrafiltration by particles and natural organic matter. Desalination and Water Treatment 18, 309-314.

Lipp, P., Baldauf, G., Schick, R., Elsenhens, K., Stebel. H. -H., 1998. Integreation of ultrafiltration to conventional drinking water treatment for a better particle removal efficiency and costs? Desalination 119, 133-142.

Lu, J., Zhang, Z., Ye, T., Qin, X., Huang, Y., Yang, X., Luo, W., Chen, B., Liu, W., Liang, Y., 2011. Aging of polyvinylchloride membranes in ultrafiltration of drinking water by chemical cleaning. IEEE.

Matosic. M., Vukovic, M., Curlin, M., Mijatovic, I., 2008. Fouling of a hollow fiber submerged membrane during long term filtration of activated sludge. Desalination 219, $57-65$.

Dube, M.A., McLean D.D., 2010. CHG 8157 - Strategies in Engineering Process Analysis - Course Notes. University of Ottawa.

Metzger, U., Le-Clech, P., Stuetz, R.M., Frimmel, F.H., Chen, V., 2007. Characterization of polymeric fouling in membrane bioreactors and the effect of different filtration modes. Journal of Membrane Science 30, 180-189.

Mendret, J., Guigui, C., Schmitz., P., Cabasssud, C., 2009. In situ dynamic characterisation of fouling under different pressure conditions during dead=end filtration: Compressibility properties of particle cakes. Journal of Membrane Science $222,20-29$

Mosqueda-Jiminez, D.B., Huck, P.M., Basu, O.D., 2008. Fouling characteristics of an ultrafiltration membrane used in drinking water treatment. Desalination 230, 79-91.

Mukerjee R., Wu, C.F.J., 2006. A Modern Theory of Factorial Designs. Springer Science and Business Media Inc. New York, UA.

Nakatsuka, S., Nakate, I., Miyani, T., 1996. Drinking water treatment by using hollow fiber membranes. Desalination 106, 55-61.

Oschmann, N., Nghiem, L.D., Schafer, A.I., 2005. Fouling mechanisms of submerged ultrafiltration membranes in greywater recycling. Desalination 179, 1-3, 215-223.

Porcelli, N., Judd,S., 2010. Chemical cleaning of potable water membranes: A review. Separation and Purification Technology, Volume 71, Issue 2, 137-143.

Puspitasari, V., Granville, A., Le Clech, P., Chen, V., 2010. Cleaning and ageing effect of sodium hypochlorite on polyvinylidene fluoride (PVDF) membrane. Separation and Purification Technology 72 (3), 301-308. 
Remize, P. J., Guigui, C., Cabassud, C. 2010. Evaluation of backwash efficiency, definition of remaining fouling and characterisation of its contribution in irreversible fouling: Case of drinking water production by air-assisted ultra-filtration. Journal of Membrane Science 355, $104-111$.

Sasaki, T., Hanaizumi, N., Minegishi, S., Henmi, M., Shimizu, Y., 2008. Characterization of PVDF hollow-fiber membrane foulants on drinking water treatment. Water Supply, Vol 8 No 5, pp 557-563.

SDWF - Safe Drinking Water Foundation. 2009. Ultrafiltration, nanofiltration and reverse osmosis. Retrieved from:

http://www.safewater.org/PDFS/knowthefacts/Ultrafiltration_Nano_ReverseOsm.pdf

Serra, C., Durand-Bourlier, L., Clifton, M.J., Moulin, P., Rouch, J-C., Aptel, P. 1999. Use of air sparging to improve backwash efficiency in hollow fiber modules. Journal of Membrane Science 161, 95-113.

Shobana, K.H. Ramya, R., and Mohan, D., 2010. Development of antifouling ultrafiltration membrane by surface modification. International Journal of Nuclear Desalination Vol 4, No. 1.

Shon, H.K., Smith, P.J., Vigneswaran, S., Ngo, H.H. 2006. Effect of hydrodynamic cleaning of a cross-flow membrane system with a novel automated approach. Desalination 202, 351-360.

Tian, J., Liang, H., Nan, J., Yang, Y., You, S., Li, G., 2009. Submerged membrane bioreactors (sMBR) for the treatment of contaminated raw water. Chemical Engineering Journal 148, 296-305.

Tian, J., Xu, Y., Chen, Z., Nan, J., Li, G., 2010. Air bubbling for alleviating membrane fouling of immersed hollow-fiber membrane for ultrafiltration of river water. Desalination 260, 225-230.

Tomaszewska, M., Mozia, S., 2002. Removal of organic matter from water by PAC/UF system. Water Research 36 (2002) 4137-4143.

USEPA, 2005. Membrane filtration guidance manual. EPA 815-R-06-009

Van der Bruggen, B., Vandecasteele, C., Gestal, T.V., Doyen, W., Leysen, R. 2003. A review of Pressure-Driven membrane processes in wastewater treatment and drinking water production. Environmental Progress Vol 22 No. 1, 46-57.

Van der Bruggen, B., Manttari, M., Nystrom, M., 2008. Drawbacks of applying nanofiltration and how to avoid them: A review. Separation and Purification Technology $63,251-263$. 
Van der Marel, P., Zwijnenburg, A., Kemperman, A., Wessling, M., Temmink, H., Van der Meer, W., 2009. An improved flux-step method to determine the critical flux and the critical flux for irreversibility in a membrane bioreactor. Journal of Membrane Science, 332 , Issues 1-2, pp. 24-29.

Wagner, J. 2001, Membrane filtration handbook - Practical tips and hints ( $2^{\text {nd }}$ Edition), Osmonics Inc. 2001. Retreived from: http://gendocs.ru/docs/30/29820/conv 1/file1.pdf

Wang, P., Wang, Z., Wu, Z., Zhou, Q., Yang, D., 2010. Effect of hypochlorite cleaning on the physiochemical characteristics of polyvinylidene fluoride membranes. Chemical Engineering Journal 162, 1050-1056.

Wolff, S.H., Zydney, A.L., 2004. Effect of bleach on the transport characteristics of polysulfone hemodialyzers. Journal of Membrane Science. 243 Issue 1-2, 389-399.

Wu, J., Le-Clech, P., Stuetz, R.M., Fane, A.G., Chen, V. 2008. Effects of relaxation and backwashing conditions on fouling in membrane bioreactor. Journal of Membrane Science 324, 26-32.

Ye, Y., Chen, V., Le-Clech, P. 2011. Evolution of fouling deposition and removal on hollow fiber membrane during filtration with periodical backwash. Desalination 283, 198-205.

Yeo, A.P.S, Law, W.K., Fane, A.G., 2006. Factors affecting the performance of a submerged hollow fiber bundle. Journal of Membrane Science 280 969-982.

Yamamura, H., Kimura. K., Watanabe, Y., 2007. Mechanisms involved in the evolution of physically irreversible fouling in MF and UF membranes used for drinking water treatment. Environmental Science Technology 41, 6789-6794.

Yigit, N. O., Civelekoglu, G., Harman, I., Koseoglu, H., Kitis, M. 2009. Effects of various backwash scenarios on membrane fouling in membrane bioreactors. Desalination 237 (2009) 346 -356.

Zularism, A.W., Ismail, A.F., Salim, R., 2006. Behaviours of natural organic matter in membrane filtration for surface water treatment - a review. Desalination 194, 211-231.

Zularism, A.W., Ahmad, A., Sakinah, M., Ismail, A.F., Matsuura, T., 2011. Role of natural organic matter (NOM), colloidal particles and solution chemistry on ultrafiltration performance. Separation and Purification Technology 78, 189-200. 


\section{APPENDICES}




\section{APPENDIX A - Zenon ZW-1 membrane specifications}

\begin{tabular}{|l|c|}
\hline Membrane Material & polyvinylidene fluoride (PVDF) \\
\hline Nominal Pore Diameter $(\mu \mathrm{m})$ & 0.04 \\
\hline Effective Membrane Surface Area (sq. m) & 0.047 \\
\hline Maximum Permeation Pressure (bar) & 0.6 \\
\hline Permeate Flow Range (mL/min) & $5-25$ \\
\hline Typical Permeate Flow (mL/min) & 15 \\
\hline Maximum Backwash Pressure (bar) & 0.7 \\
\hline Maximum Air Flow for Scouring (LPM) & 30 \\
\hline Operating pH Range & $5-9$ \\
\hline Cleaning pH Range & $2-10.5$ \\
\hline $\begin{array}{l}\text { Maximum Operating/Cleaning Temperature } \\
\left({ }^{\circ} \mathrm{C}\right)\end{array}$ & 40 \\
\hline $\begin{array}{l}\text { Minimum sodium hypochlorite (200 ppm) Soak } \\
\text { Time (hrs) }\end{array}$ & 5 \\
\hline \begin{tabular}{l} 
Minimum citric acid (2-3 pH) Soak Time (hrs) \\
\hline
\end{tabular} & 5 \\
\hline
\end{tabular}




\section{APPENDIX B - Detailed schematics of flow lines (air, feed and}

permeate)

\begin{tabular}{|c|l|}
\hline \multicolumn{2}{|l|}{ Air Flow Line: } \\
\hline 1 & Flow Modulator \\
\hline 2 & Masterflex High-Performance Precision Norprene Tubing (1/4"ID) \\
\hline 3 & Whatman HEPA-VENT ${ }^{\text {TN }}$ \\
\hline 4 & Cole-Parmer C-Flex Tubing (1/4"ID x 3/8"OD) \\
\hline $5 a$ & Cole-Parmer Polypropylene Straight Union (3/8"OD) \\
\hline $5 b$ & $\begin{array}{l}\text { Cole-Parmer Polypropylene Compression Reducing Unions (1/4"ID x } \\
3 / 8 " O D)\end{array}$ \\
\hline $6 a$ & Cole-Parmer FEP Rigid Tubing (1/4"ID x 3/8"OD) \\
\hline $6 b$ & Cole-Parmer FEP Rigid Tubing (3/16"ID x 1/4"OD) \\
\hline $7 a$ & Differential Pressure Technologies Electronic Flow meter (20 LPM) \\
\hline $7 b$ & AALBORG GFM37 Mass Flow meter (0-15 LPM) \\
\hline 8 & Valve \\
\hline 9 & Boyu 20cm Air Curtain \\
\hline 10 & ZeeWeed ZW1 - Membrane Module \\
\hline Note: a used in Setup 1; b used in Setup 2 \\
\hline
\end{tabular}




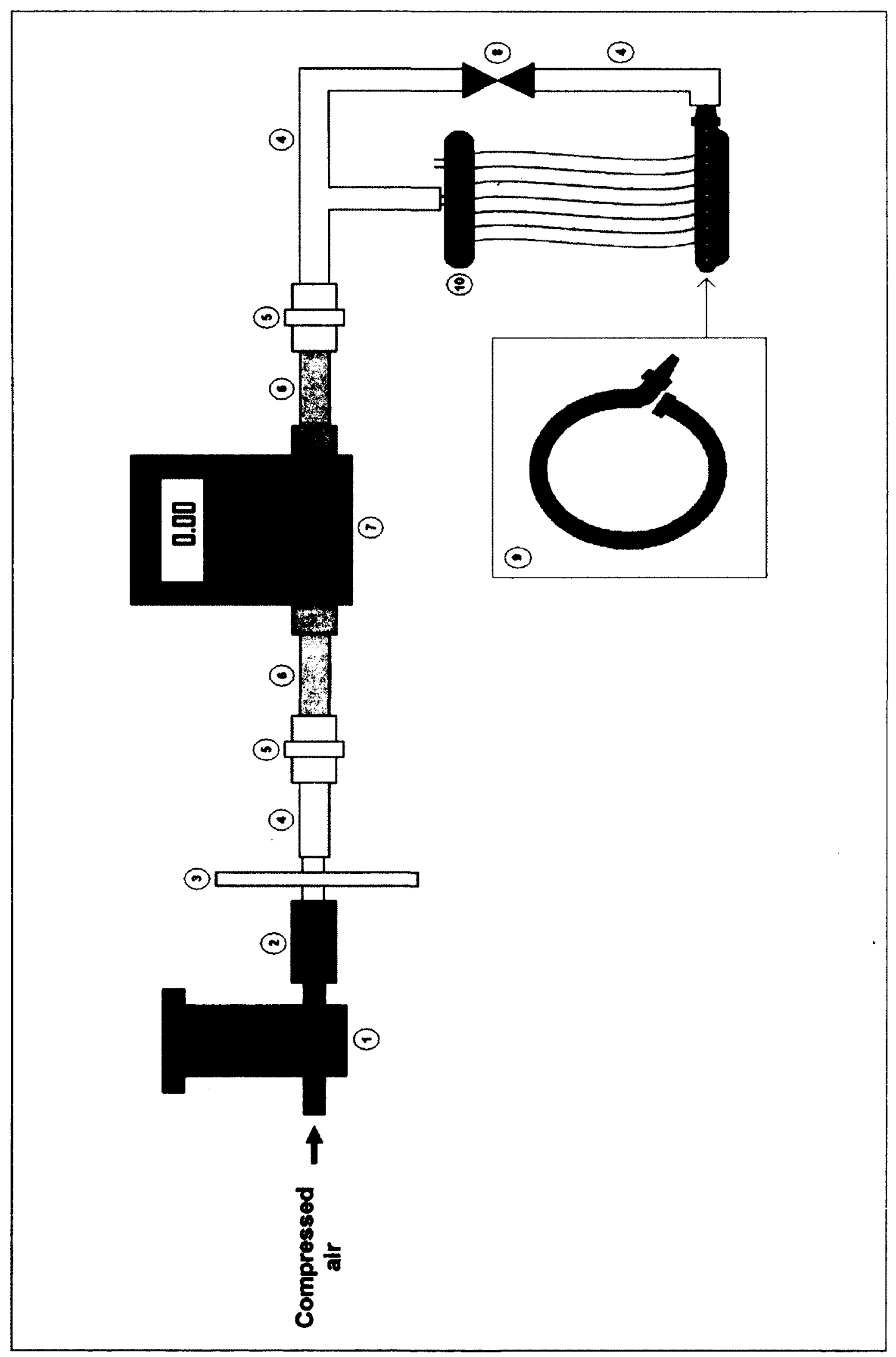




\begin{tabular}{|c|l|}
\hline \multicolumn{2}{|c|}{ Feed Flow Line: } \\
\hline 1 & Nalgene Heavy Duty Cylindrical Tank - Feed Tank (50 L) \\
\hline 2 & Cole-Parmer C-Flex Tubing (1/4"ID x 3/8"OD) \\
\hline 3 & Masterflex Polypropylene Straight Connector (1/4"ID) \\
\hline 4 & Masterflex High-Performance Precision Norprene Tubing (1/4"ID) \\
\hline 5 & Masterflex Peristaltic Pump (model \# 7024-20) \\
\hline 6 & Plexiglass Reactor Tank (3 L) \\
\hline
\end{tabular}




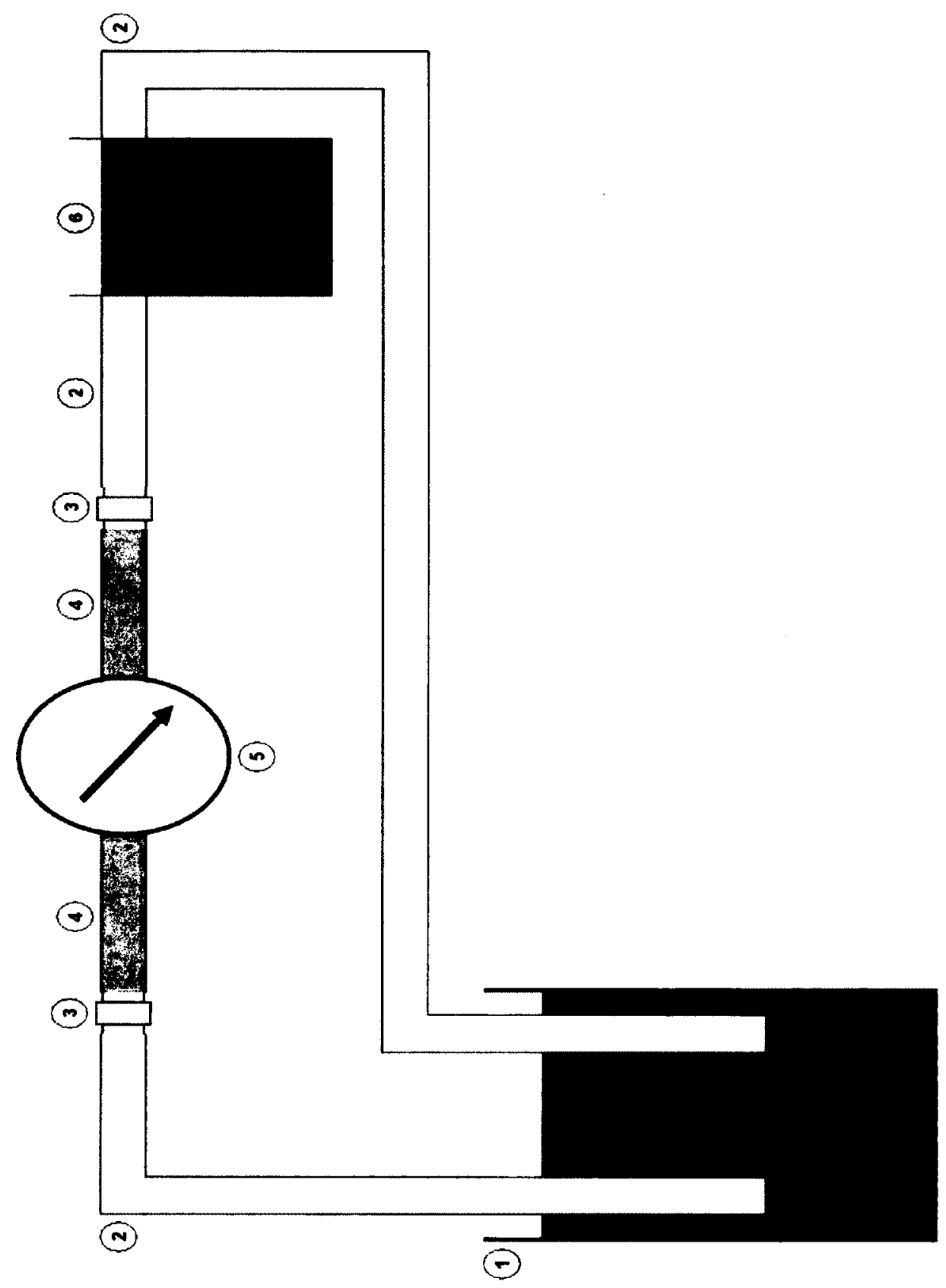




\begin{tabular}{|c|c|}
\hline \multicolumn{2}{|r|}{ Permeate Flow Line: } \\
\hline 1 & ZeeWeed ZW1 - Membrane Module \\
\hline 2 & Masterflex High-Performance Precision C-flex Tubing (3/8"ID) \\
\hline 3 & Cole-Parmer Polypropylene Union T-connector (3/8"OD) \\
\hline 4 & Cole-Parmer FEP Rigid Tubing (1/4"ID × 3/8"OD) \\
\hline 5 & Swagelok Stainless Female Tee (SS-600-3TTF) \\
\hline 6 & Cole-Parmer 68075 Pressure Transducer (-14.7 to $15 \mathrm{psig})$ \\
\hline 7 & $\begin{array}{l}\text { Cole-Parmer Polypropylene Compression Reducing Unions (3/8"ID x } \\
1 / 2 \text { "OD) }\end{array}$ \\
\hline 8 & Masterflex High-Performance Platinum Cured Silicone Tubing (1/4"ID) \\
\hline 9 & Valve \\
\hline 10 & Glass AirMater Tank \\
\hline 11 & Teflon Pierceable Cap \\
\hline 12 & $\begin{array}{l}\text { Masterflex L/S Digital Peristaltic Pump System with Easy-Load } 3 \\
\text { Pump Head } 77800-62 \text { for High-Performance Precision Tubing }\end{array}$ \\
\hline 13 & Rubber Stopper \\
\hline 14 & Plastic Filter Flask ( $1 \mathrm{~L})$ \\
\hline 15 & Cole-Parmer C-Flex Tubing (1/4"ID x 3/8"OD) \\
\hline
\end{tabular}




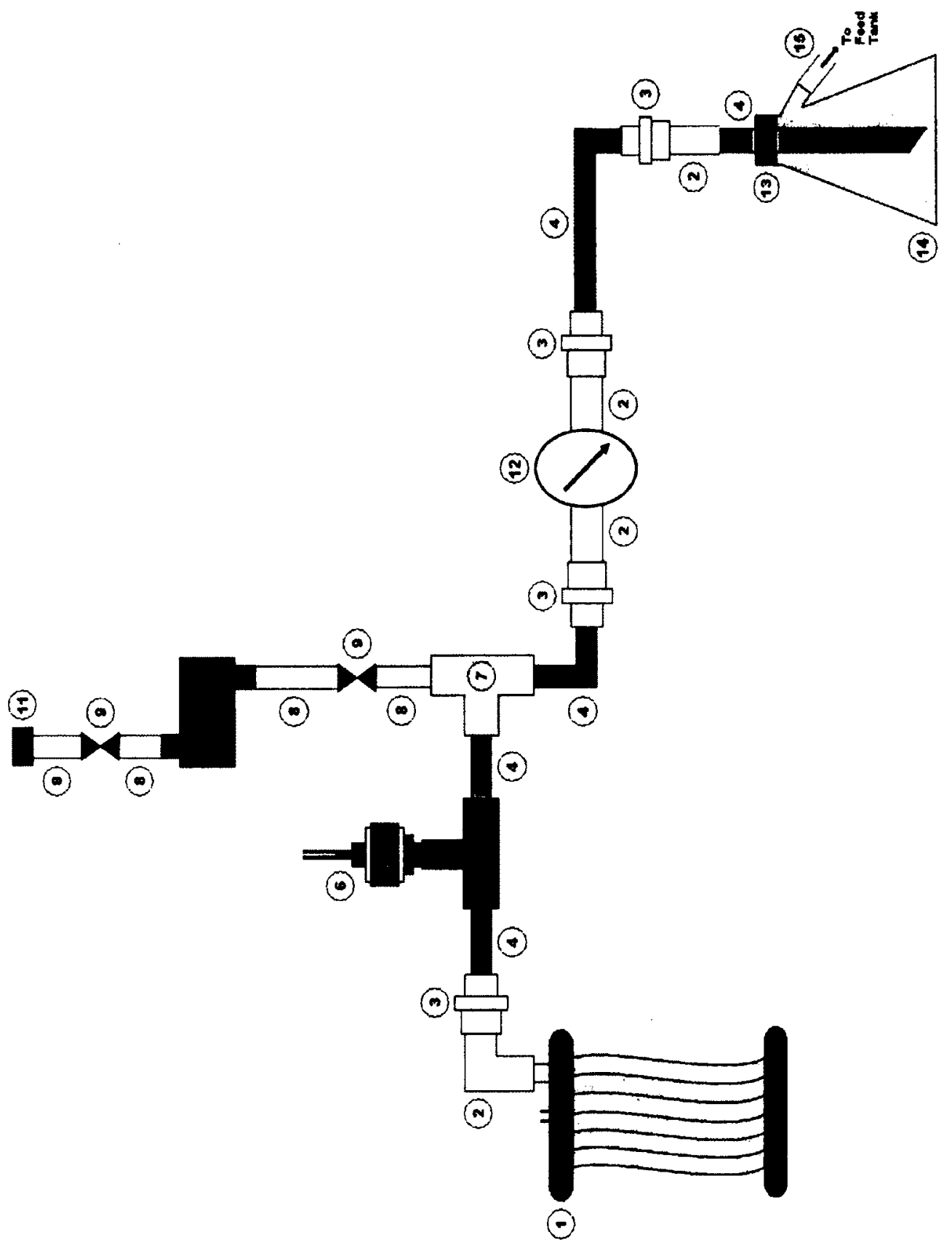




\title{
APPENDIX C - Operation Flux 2.0 Visual Basic Code
}

\author{
Imports Advantech. Adam
}

Imports Advantech.Common

- Authors: Brian Alder (B.Eng) and Nigel P. De Souza (B.Eng)

Public Class Form 1

' $\star \star *$ DECLARE FORM VARIABLES $* \star \star \prime$

- The Timer Interval in seconds

Dim nTimerInterval As Integer $=500$

- UTC Time at start of recording

Dim nstartTimeofRecording As Long $=0$

Dim nstartTimeofRecording2 As Long $=0$

- The UTCTime of when the Timer Started

Dim nLastRecordingEvent As Long $=0$

Dim nLastRecordingEvent2 As Long $=0$

'Timer Conversion Factors

Dim nsecondsToUTC As Long $=10000000$

- The folder where the save files will be written to

Dim sSaveFolder As String = ""

Dim sSaveFolder2 As String = " "

- The path name of where to save the Recording File and Log File Dim sRecordingFilePathName As string = " "

Dim sLogFilePathName As String = " "

Dim sRecordingFilePathName2 As String = ""

Dim sLogFilePathName2 As String = "

- The Names of the Output Files

Dim sRecordingFileName As String = "\Recording.txt"

Dim sLogFileName As String = "\Log.txt"

- The Header Line for the Save File

Dim sHeaderLine As String = "TIMESTAMP, ELAPSED_SECONDS,

PUMP_SETTING, PUMP_DIRECTION, FLOW_METER_READING, PRESSURE_READING,

FLUX, TEMPERATURE"-

- The Number of entries into the Log File

Dim nLogentries As Integer $=0$

Dim nLogentries2 As Integer $=0$ 
- The string containing the last read characters from the Flow Meter Serial Port

Dim sflowMeterReading As String = "

- The last recieved complete string received from the Flow Meter Serial Port.

Dim SFlowMeterDisplay As String =" "

- The last read Pressure Reading from the ADAM Serial Port

Dim sPressureReading As String = " "

Dim sPressureReading2 As String = "

Dim sFlowReading2 As String $=" "$

- The MA to PSIG Conversion factor

Dim nPressureConversion As Double $=1.8563$

- The Pressure offset

Dim nPressureoffset As Double $=22.125$

- The $m A$ to $\mathrm{L} / \mathrm{min}$ Conversion factor

Dim nFlowConversion As Double $=0.9375$

- The Flow offset

Dim $n$ Flow

- Integer value representing the Forward / Backward Flow in $\mathrm{mL} / \mathrm{min}$ of the Pump

Dim nFlowsettingFF As Integer $=0$

Dim nFlowsettingFF2 As Integer $=0$

Dim nFlowsettingBF As Integer $=0$

Dim nFlowsettingBF2 As Integer $=0$

- Number of Forward / Backward cycles to happen before a relax

Dim nPumpRelaxCycles As Integer $=0$

Dim nPumpRelaxcycles2 As Integer $=0$

- Number of cycles Completed

Dim ncyclecount As Integer $=0$

Dim ncyclecount2 As Integer $=0$

- Integer value representing the Forward Flow / Backward Flow / Relaxation Duration in Seconds of the Pump

Dim nDurationfF As Integer $=0$

Dim nDurationFF2 As Integer $=0$

Dim nDurationBF As Integer $=0$

Dim nDurationBF2 As Integer $=0$

Dim nDurationRL As Integer $=0$

Dim nDurationRL2 As Integer $=0$

- The Logging Interval for the Pump Modes In Seconds

Dim nLogIntervalinsecondsFE As Integer $=0$

Dim nLogIntervalinsecondsFF2 As Integer $=0$ 


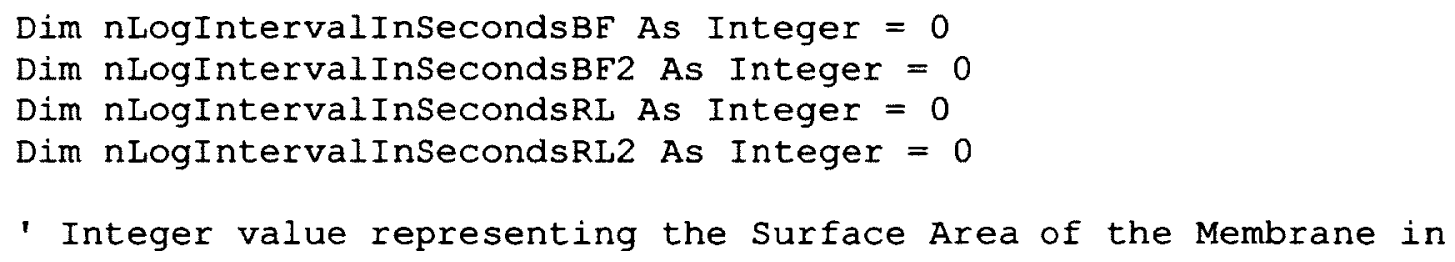

Private Sub Form1_Load(ByVal sender As System.Object, ByVal e As System.EventArgs) Hand̄les MyBase.Load

- Create the column headers.

ListViewl.Columns.Add("Timestamp", 140) 


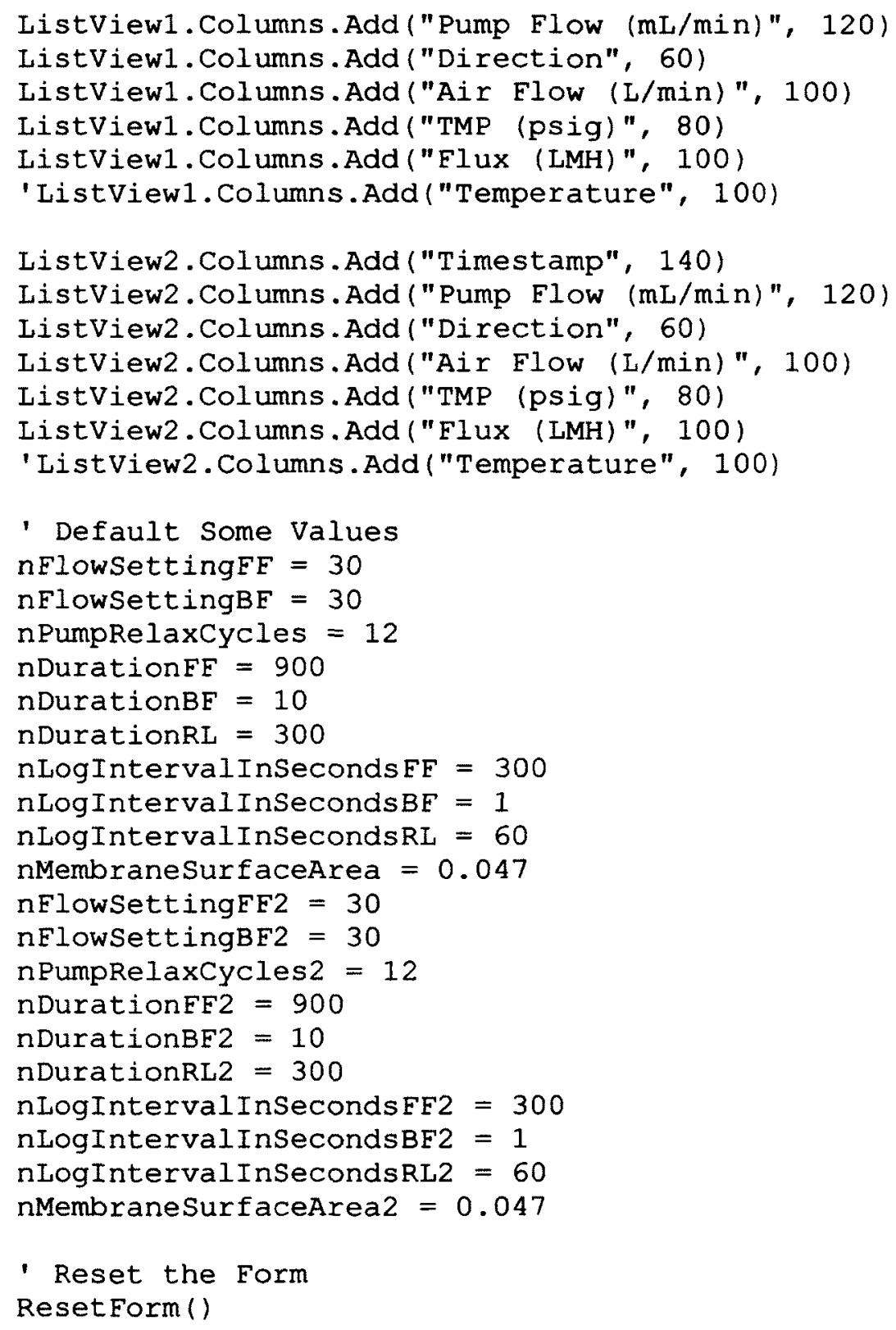

End Sub

Private Sub ButtonReset Click(ByVal sender As System.Object, Byval e As System.EventArgs) Handles ButtonReset.Click

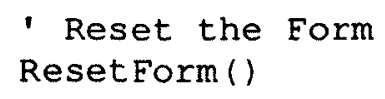

End Sub

Private Sub ResetForm()

- Clear the Log

ListBoxLog. Items.Clear() 


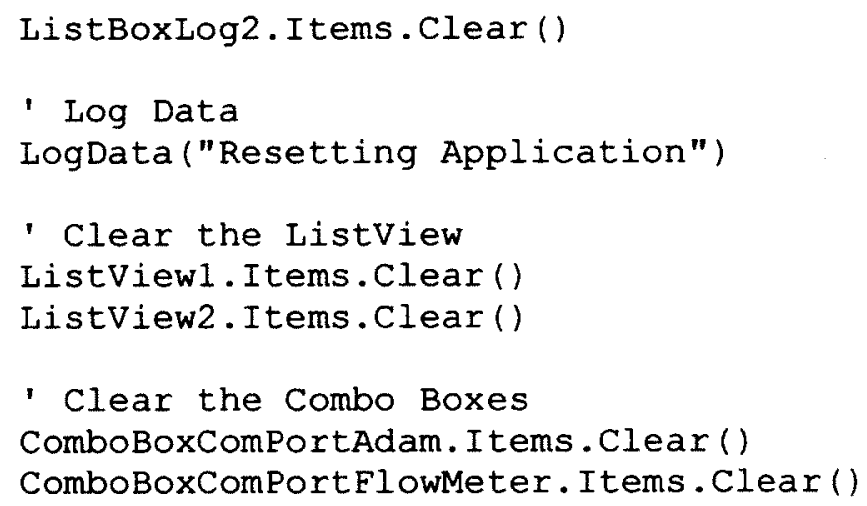


TextBoxForwardFlowDuration. Text $=\operatorname{Str}$ (nDurationFF)

LogData("Setting Forward Flow Duration To " +

Str(nDurationfF) + " Seconds")

TextBoxForwardFlowDuration2. Text $=\operatorname{str}($ nDurationFF2)

LogData2("Setting Forward Flow Duration To " +

Str(nDurationFF2) + " Seconds")

- Default the Forward Flow Log Interval

TextBoxLogIntervalFF. Text $=$ Str (nLogIntervalinsecondsFF)

LogData("Setting Forward Flow Log Interval To " +

Str (nLogIntervalinSecondsFF) + "Seconds")

TextBoxLogIntervalFF2. Text = Str(nLogIntervalInSecondsFF2)

LogData2("Setting Forward Flow Log Interval To " +

Str(nLogIntervalinSecondsFF2) + "Seconds")

- Default the Backward Flow

TextBoxBackwardFlow. Text $=\operatorname{Str}$ (nFlowSettingBF)

LogData("Setting Backward Flow of Pump To " +

Str (nFlowSettingBF) + " $\mathrm{mL} / \mathrm{min} ")$

TextBoxBackwardFlow2. Text = Str (nFlowsettingBF2)

LogData2("Setting Backward Flow of Pump To " +

Str(nFlowsettingBF2) + " $\mathrm{mL} / \mathrm{min} ")$

- Default the Backward Flow Duration

TextBoxBackwardFlowDuration. Text = Str (nDurationBF)

LogData("Setting Backward Flow Duration To " +

Str(nDurationBF) + "Seconds")

TextBoxBackwardFlowDuration2. Text = Str (nDurationBE2)

LogData2("Setting Backward Flow Duration To " +

Str(nDurationBF2) + " Seconds")

- Default the Backward Flow Log Interval

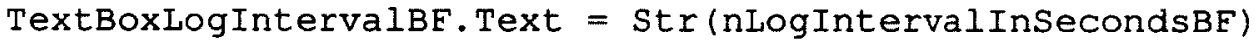

LogData("Setting Backward Flow Log Interval To " +

Str(nLogIntervalinsecondsBF) + " Seconds")

TextBoxLogIntervalBF2. Text $=\operatorname{Str}($ nLogIntervalinSecondsBF2)

LogData2("Setting Backward Flow Log Interval To " +

Str(nLogIntervalinSecondsBF2) + " Seconds")

'Default the Relax Cycles

TextBoxRelax. Text $=$ Str (nPumpRelaxCycles)

LogData("Setting Relax Cycles To " + Str(nPumpRelaxCycles))

TextBoxRelax2. Text = Str (nPumpRelaxCycles2)

LogData2("Setting Relax Cycles To " +

Str (nPumpRelaxCycles2))

'Default the Relax Duration

TextBoxRelaxDuration. Text $=$ Str $($ nDurationRL)

LogData("Setting Relax Duration To " + Str(nDurationRL) + "

Seconds")

TextBoxRelaxDuration2. Text = Str (nDurationRL2)

LogData2("Setting Relax Duration To " + Str(nDurationRL2) +

" Seconds") 


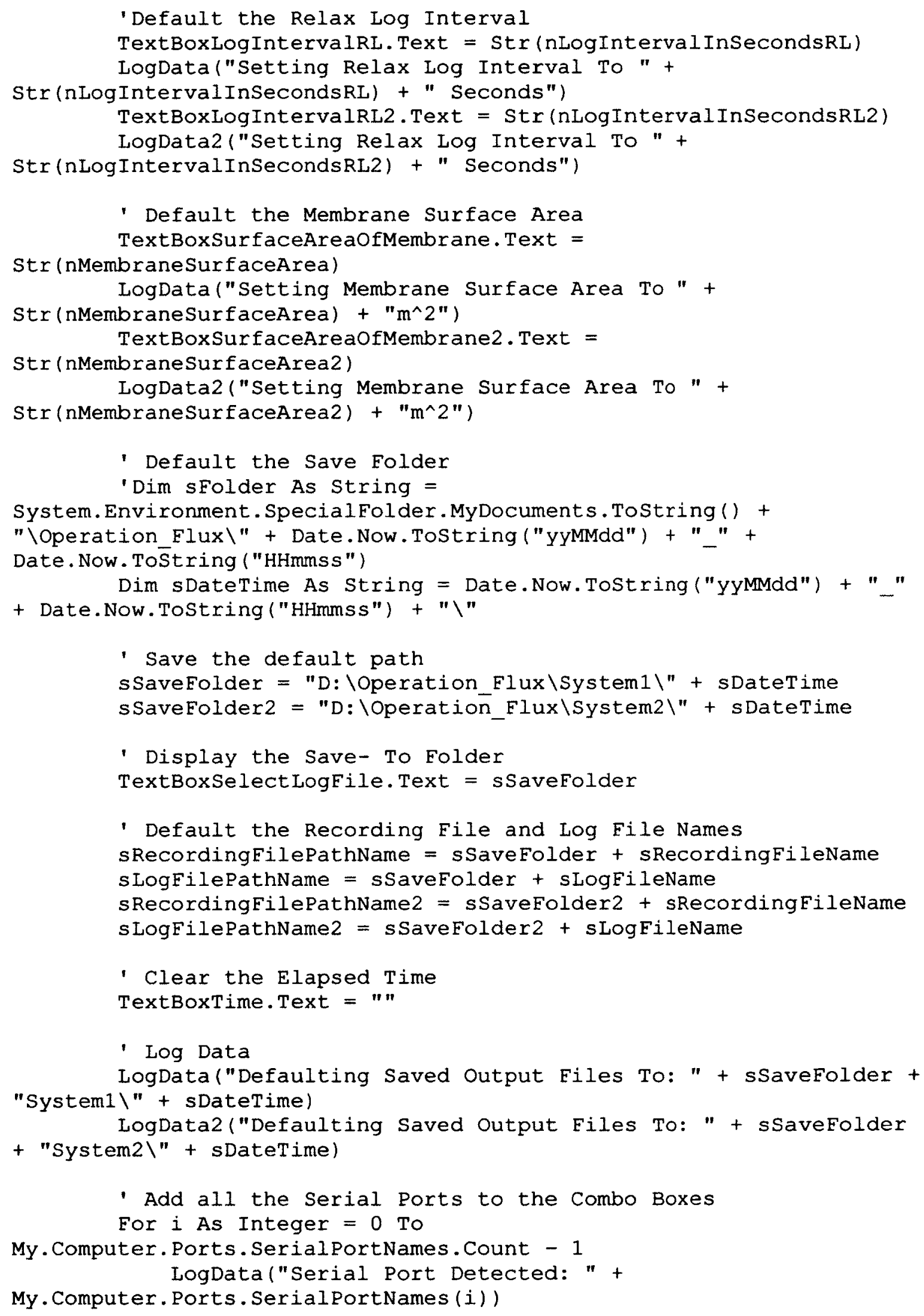


LogData2 ("Serial Port Detected: " +

My. Computer.Ports.SerialPortNames (i))

ComboBoxComPortAdam. Items.Add (My. Computer.Ports.SerialPortNames (i))

ComboBoxComPortFlowMeter. Items. Add (My . Computer. Ports. Serial Port Names

(i) )

Next

End Sub

Private Sub ButtonstartRecording_Click(ByVal sender As System.Object, ByVal e As System.EventArgs) Handles

ButtonstartRecording.Click

- Start the Recording

StartRecording ()

End Sub

Private Sub ButtonstopRecording_Click(ByVal sender As System.Object, ByVal e As System.EventArgs) Handles

ButtonstopRecording.Click

- Stop Recording

StopRecording ()

End Sub

Private Sub startRecording()

- Ensure the Recording File is valid

If sRecordingFilePathName = " " Or sRecordingFilePathName2 =

" "Then

- Display Error Box.

' Note: This should NEVER happen. The Recording File

Name is defaulted and

- The user can only make a change to a valid existing

folder.

MsgBox("Please Select A Folder To Save To.")

- Prompt the user to select a folder to save output

files to

GetSavetoFolder( )

Else

- Extract all the Pump and Membrane Data (1)

Dim nEF As Integer = Val (TextBoxForwardFlow. Text)

Dim nFED As Integer =

Val (TextBoxForwardElowDuration. Text)

Dim nEFL As Integer = Val (TextBoxLogIntervalFF. Text)

$\operatorname{Dim} \mathrm{nBF}$ As Integer $=\operatorname{Val}$ (TextBoxBackwardFlow. Text) 


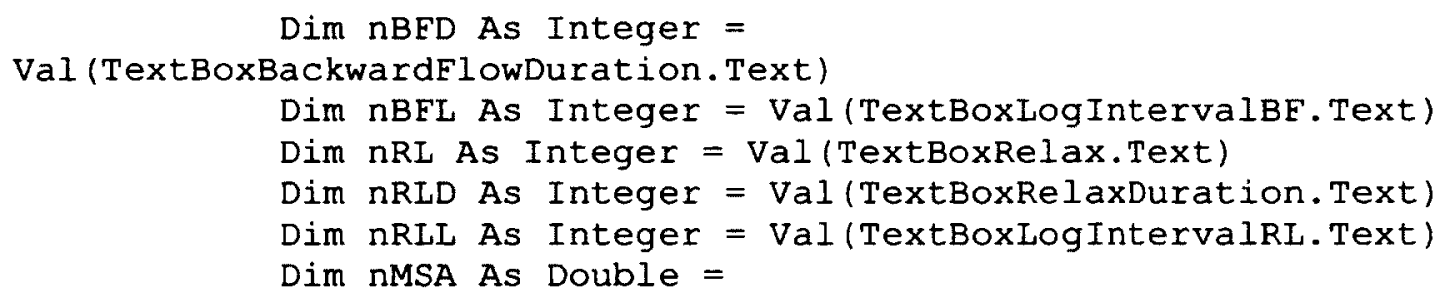




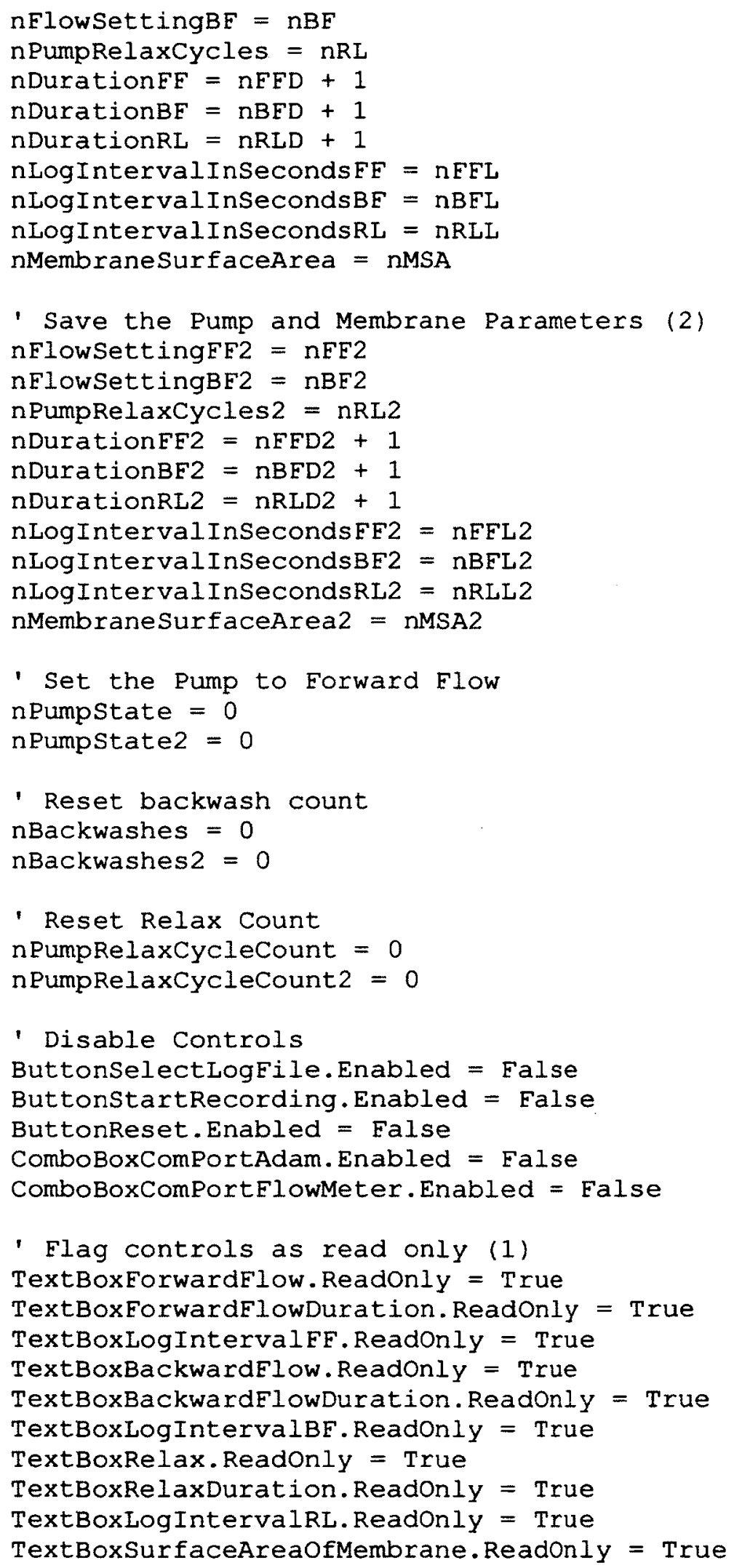




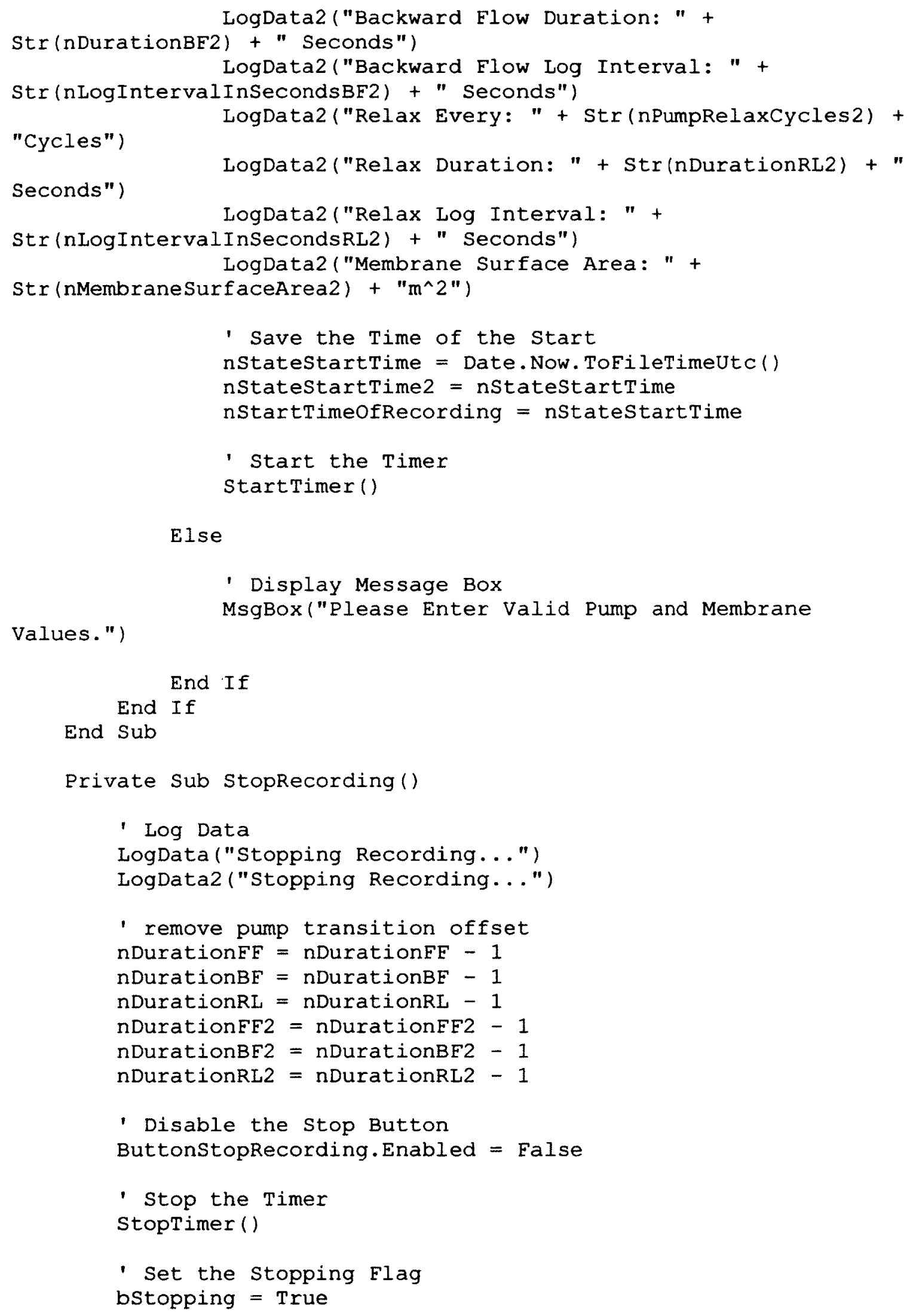




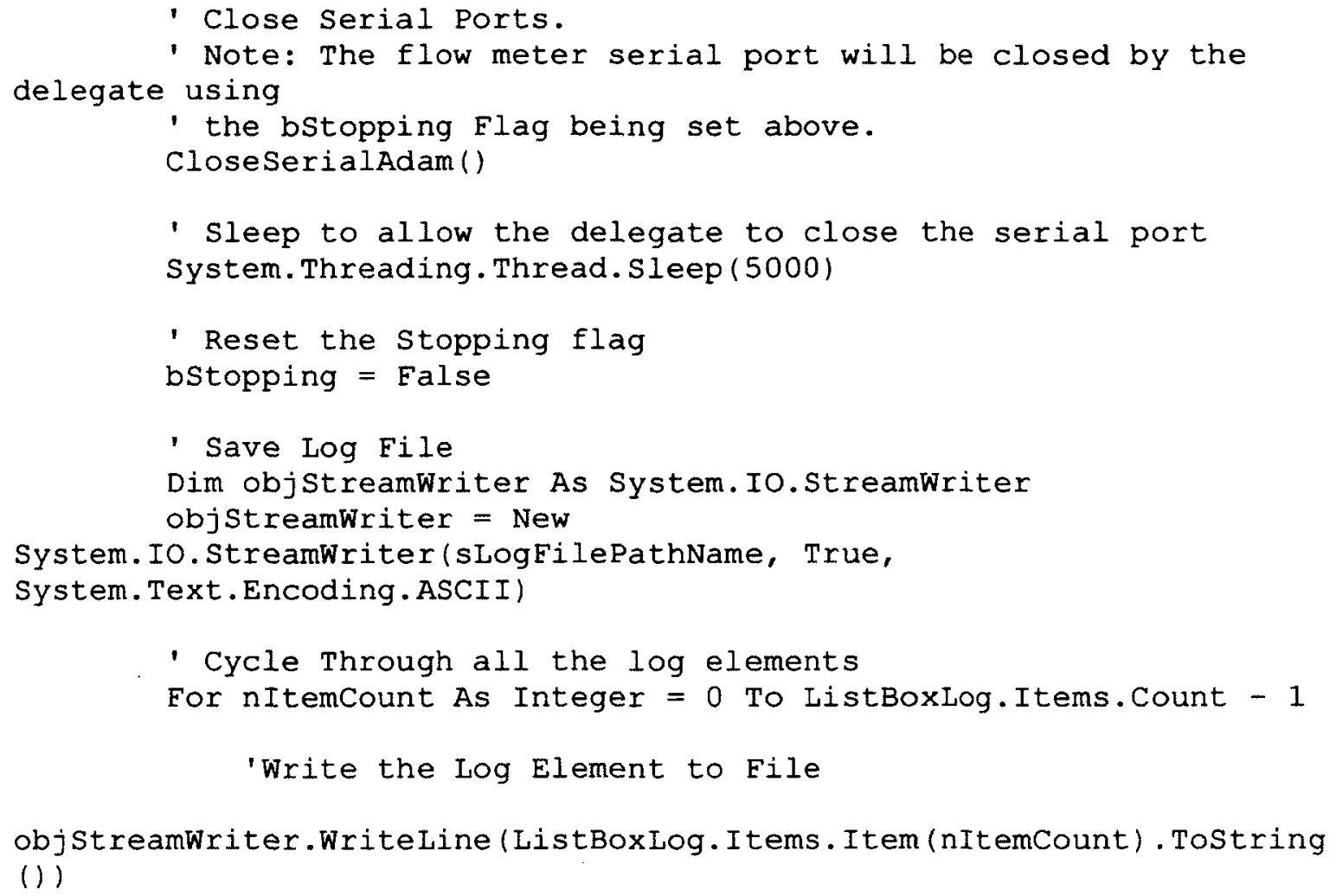




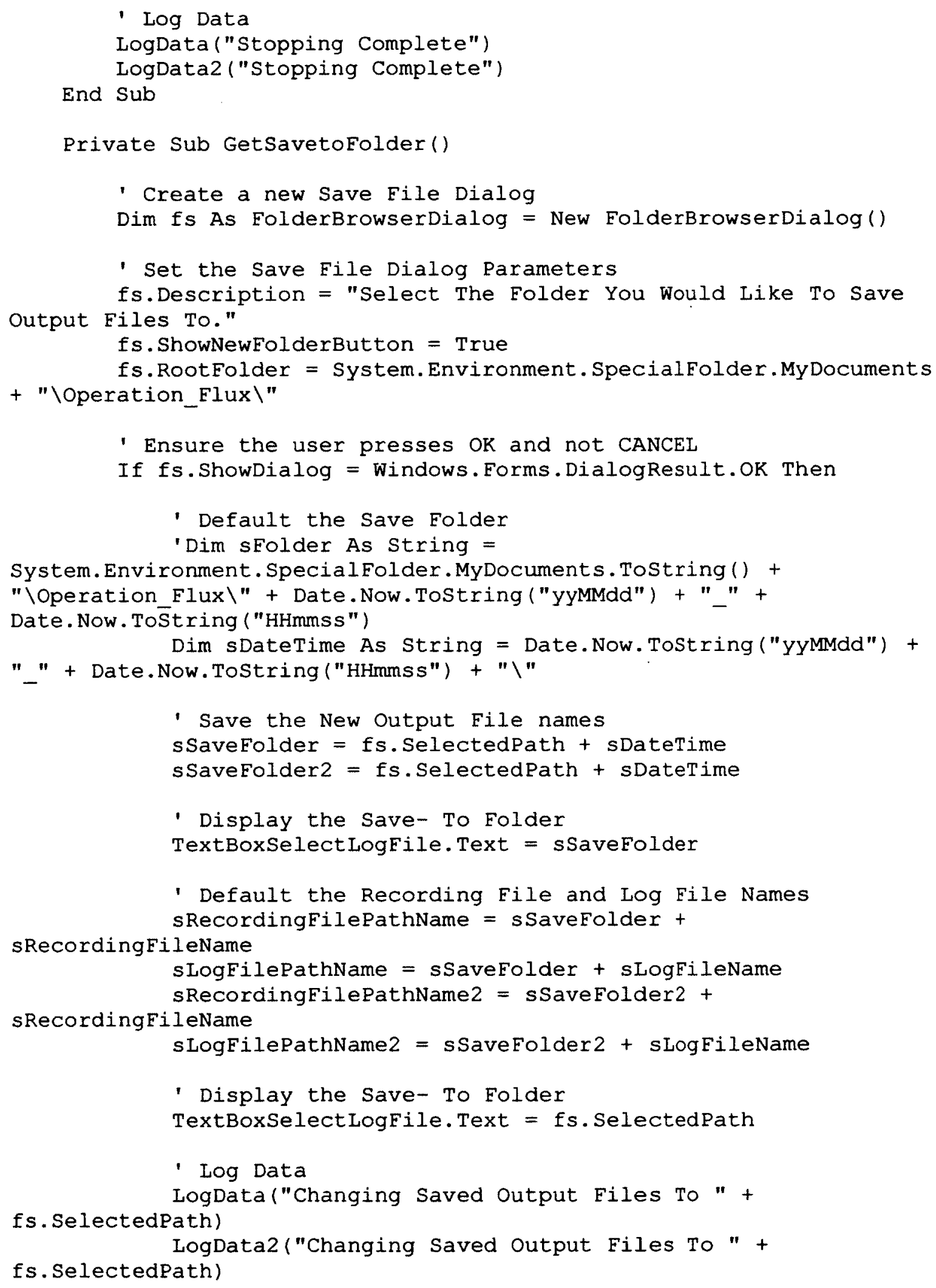


Private Sub ButtonselectlogFile_Click(ByVal sender As System.Object, ByVal e As System.EventArgs) Handles ButtonselectLogFile.Click

- Prompt the user to select a folder to save output files to GetSavetoFolder()

End Sub

Private Sub StartTimer()

- Set the Timer Interval

Timerl. Interval $=\mathrm{n}$ TimerInterval

- Start the Timer

Timer1.Start()

- Log Data

LogData("Starting Timer: Every " + Str(nTimerInterval) + " Milliseconds")

LogData2("Starting Timer: Every " + Str(nTimerInterval) + " Milliseconds")

End Sub

Private Sub StopTimer()

- Stop the Timer

Timer1.Stop()

- Log Data

LogData("Stopping Timer")

LogData2("Stopping Timer")

End Sub

Private Sub Processsystem1 (ByVal nsystemTime As Long, ByVal nUTCDeltaseconds As Long)

- Flag to determine if we need to log

Dim bMustLog As Boolean = False

- Check to see if we are currently in Forward Flow $(0)$,

Backward Flow (1), or Relax (2)

If nPumpstate $=0$ Then

' Check to see if the log interval has elapsed

If (nLogIntervalinSecondsFF > 0) And (nSystemTime >

(nLastRecordingEvent + (nLogIntervalinSecondsFF * nSecondsToUTC)) )

Then

'Flag that we must log

bMustLog $=$ True 
End If

' Check to see if transition time has elapsed

If nsystemTime > (nstatestartTime + (nDurationFF *

nSecondsToUTC)) Then

- Save the State Start Time

nstatestart $\mathrm{Time}=$ nsystemTime

'Check to see if we have Backward Flow Duration

If nDuration $\mathrm{BF}>0$ Then

- Update the Pump State

n Pumpstate $=1$

- Log Data

Elow.")

LogData("Changing Pump State To: Backward

1, True)

- Increment the Backwash Count

nBackwashes $=$ nBackwashes +1

- Log Data

LogData("Backwash Count: " + Str(nBackwashes)) ListBoxLog. SetSelected (ListBoxLog. Items. Count -

Else

' Increment the number of cycles

nCyclecount $=$ nCyclecount +1

' Check to see if we have a Relax Duration

If nDurationRL $>0$ And nCyclecount =

nPumpRelaxcycles Then

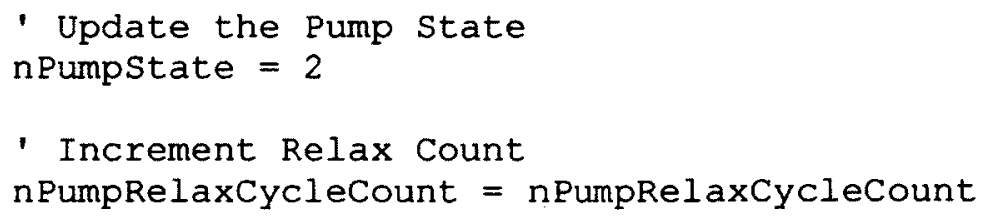

Else

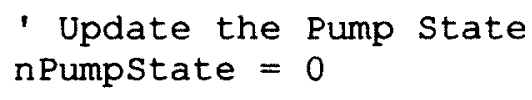




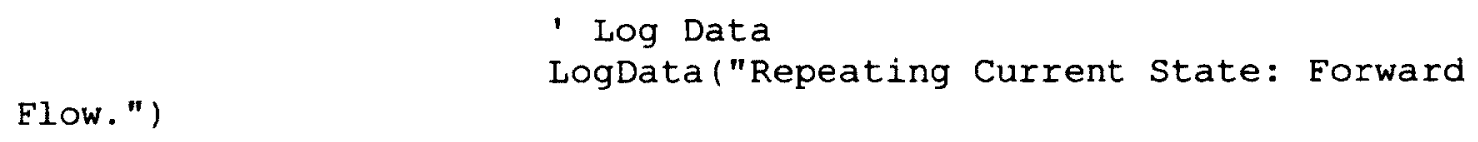

End If

' Check to see if transition time has elapsed

Else

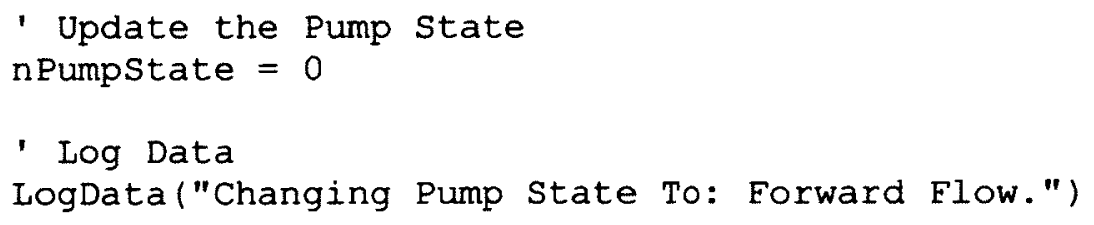


End If

End If

ElseIf nPumpstate $=2$ Then

' Check to see if the log interval has elapsed

If (nLogIntervalinSecondsRL > 0) And (nSystemTime >

(nLastRecordingEvent + (nLogIntervalinSecondsRL * nSecondsToUTC)) ) Then

'Flag that we must log

bMustLog $=$ True

End If

'Check to see if transition time has elapsed

If nsystemTime > (nstatestartTime + (nDurationRL *

nSecondsToUTC)) Then

- Reset Cycle Count

ncyclecount $=0$

- Save the State start Time

nstatestartTime $=$ nsystemTime

- Update the Pump state

nPumpstate $=0$

- Log Data

LogData("Changing Pump State To: Forward Flow.")

End If

End If

- Check and see if its time to record new data to the log If bMustLog $=$ True Then

- Save the Current UTC Timestamp

nLastRecordingEvent $=$ nSystemTime

- Determine Current Pump Setting and Direction and flux Dim sPumpsetting As String = ""

Dim sPumpDirection As String = " "

Dim nflux As Double $=0$

- Check the Pump State

If nPumpstate $=0$ Then

- Set the Pump Direction String

sPumpDirection = "Forward" 


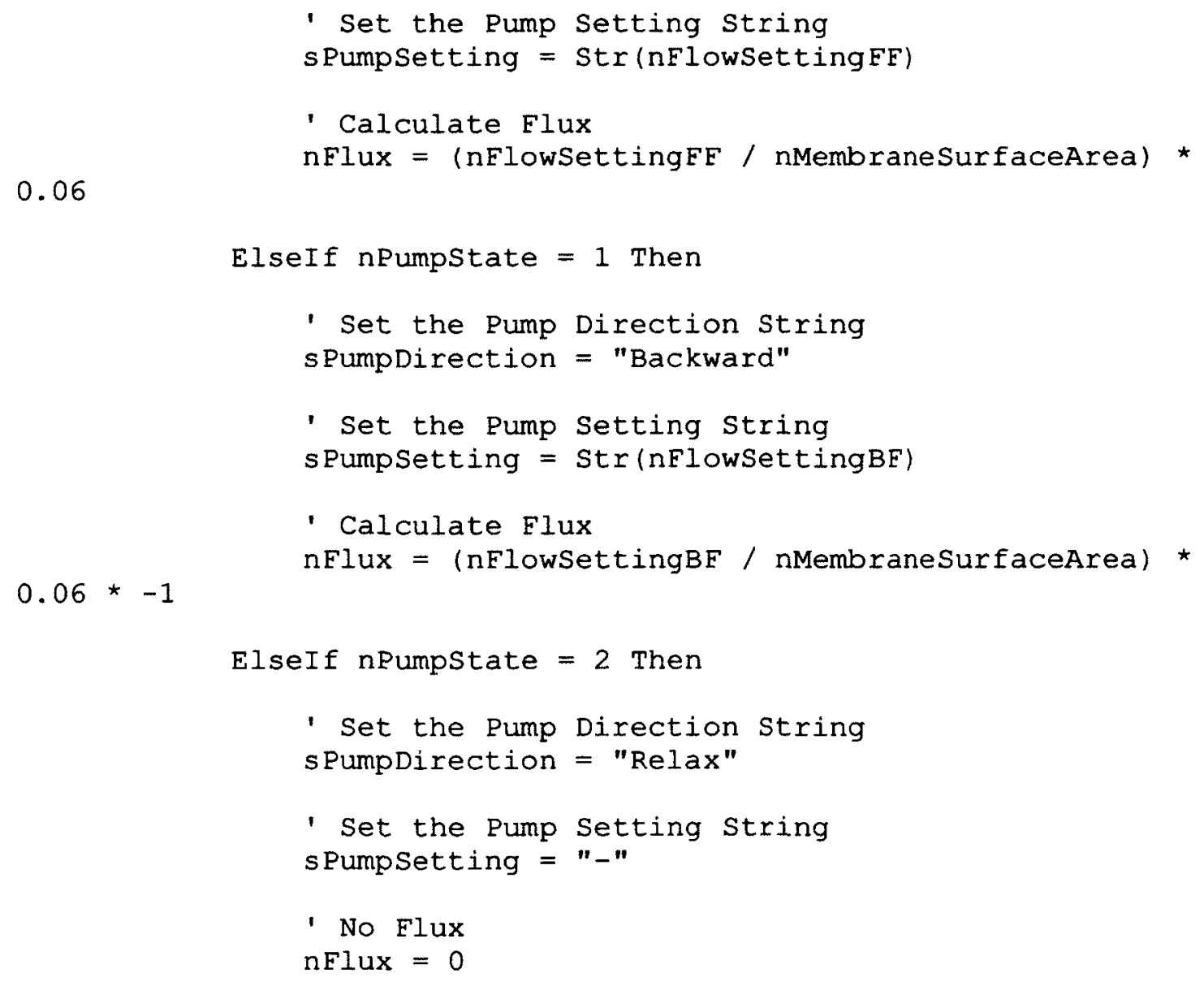

- Check to see if we've hit the max allowed in the list If ListView1. Items. Count $=14$ Then

- Remove the oldest item from the list ListView1. Items. RemoveAt (0)

End If

- Create a new string array to hold the recorded values Dim sListitems(6) As string

Dim itm As ListViewItem

sListitems $(0)=$ Date. Now

sListItems $(1)=$ sPumpsetting

sListItems $(2)=$ sPumpDirection

slistItems $(3)=$ sFlowMeterDisplay ' handled by delegate

ReadAdamValue sListItems $(4)=$ sPressureReading ' handled by

sListItems (5) = nFlux.Tostring("F03")

'sListItems $(6)=$ "Room"

Default For Now

itm = New ListViewItem (sListItems)

ListViewl. Items. Add (itm) 
- Increment the number of

nLogEntries $=$ nLogEntries +1

- Open the file to write to and write the data

Dim objstreamwriter As System.Io.Streamwriter

objstreamwriter $=$ New

System.Io.StreamWriter(sRecordingFilePathName, True,

System. Text.Encoding. ASCII)

- Create the string to write to file

Dim sLine As String = sListItems $(0)+", "+$

Str(nUTCDeltaSeconds) + ", + sListItems (4) + "," + sListItems (1) + $", "+$ sListItems $(2)+", "+\operatorname{sListItems}(3)+", "+\operatorname{sListItems}(5)+$

", + "Room"

- Remove all spaces from the string

Dim sNewLine As String = " "

For $i$ As Integer $=0$ To sLine. Length -1

If sLine.Substring(i, 1) $<$ " "Then End If sNewLine $=$ sNewLine + sLine.Substring $(i, 1)$

Next

- Write the line to file

objstreamwriter.WriteLine (sNewLine)

End If

objStreamWriter.Close()

End Sub

Private Sub ProcessSystem2(ByVal nsystemTime As Long, ByVal nUTCDeltaseconds As Long)

- Elag to determine if we need to log

Dim bMustLog As Boolean = False

- Check to see if we are currently in Forward Flow (0), Backward Flow (1), or Relax (2)

If nPumpstate $2=0$ Then

'Check to see if the log interval has elapsed

If (nLogIntervalinsecondsFF2 > 0) And (nSystemTime >

(nLastRecordingEvent $2+($ nLogIntervalinsecondsFF2 * nSecondsToUTC))) Then

' Flag that we must log

bMustLog $=$ True

End If

'Check to see if transition time has elapsed

If nsystemTime > (nstatestartTime2 + (nDurationFF2 *

nSecondsToUTC)) Then

- Save the State Start Time 
End If

ElseIf nPumpstate2 $=1$ Then

' Check to see if the log interval has elapsed

If (nLogIntervalinsecondsBF2 > 0) And (nsystemTime >

(nLastRecordingEvent2 + (nLogIntervalinsecondsBF2 * nsecondsToUTC))) Then

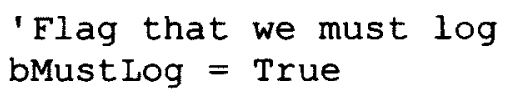

End If

' Check to see if transition time has elapsed

If nsystemTime > (nstatestartTime2 + (nDurationBF2 *

nSecondsToUTC)) Then

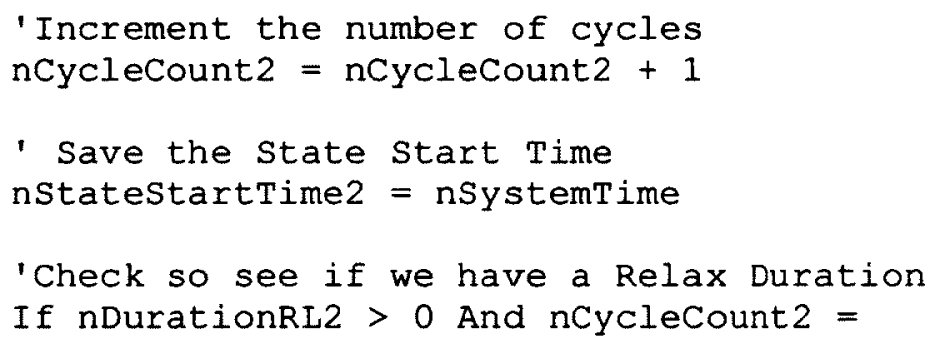

1

- Log Data

LogData2("Changing Pump state To: Relax.")

LogData2("Relax Count: " +

Str (nPumpRelaxCycleCount2))

Else

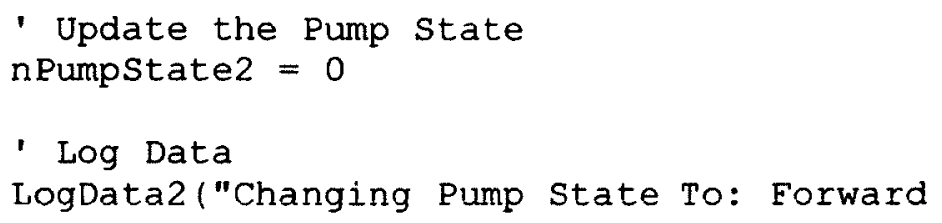

End If

End If

ElseIf nPumpState2 $=2$ Then 


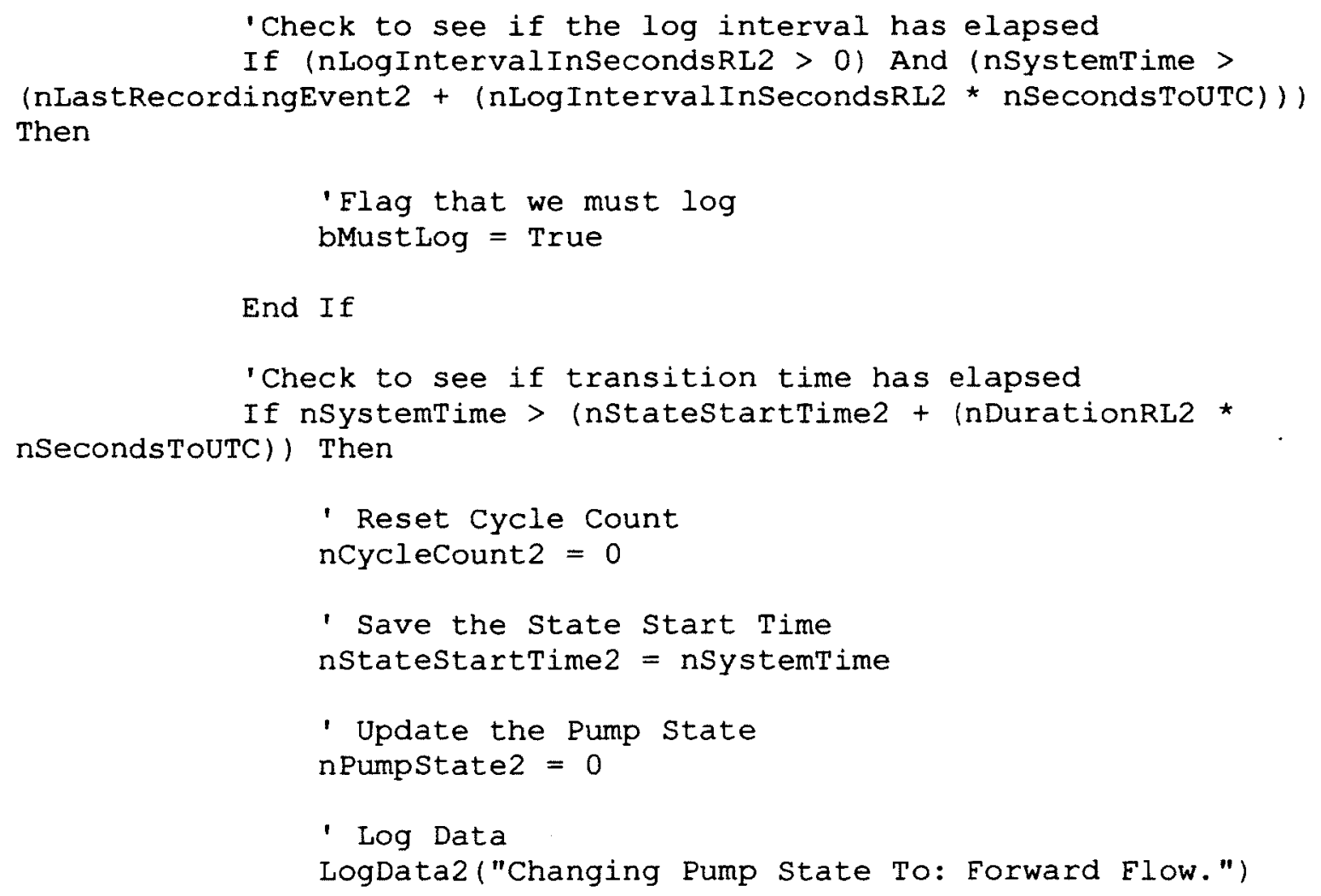

End If

' Check to see if transition time has elapsed

If nsystemTime > (nstatestartTime2 + (nDurationRL2 * nSecondsToUTC)) Then

End If

End If

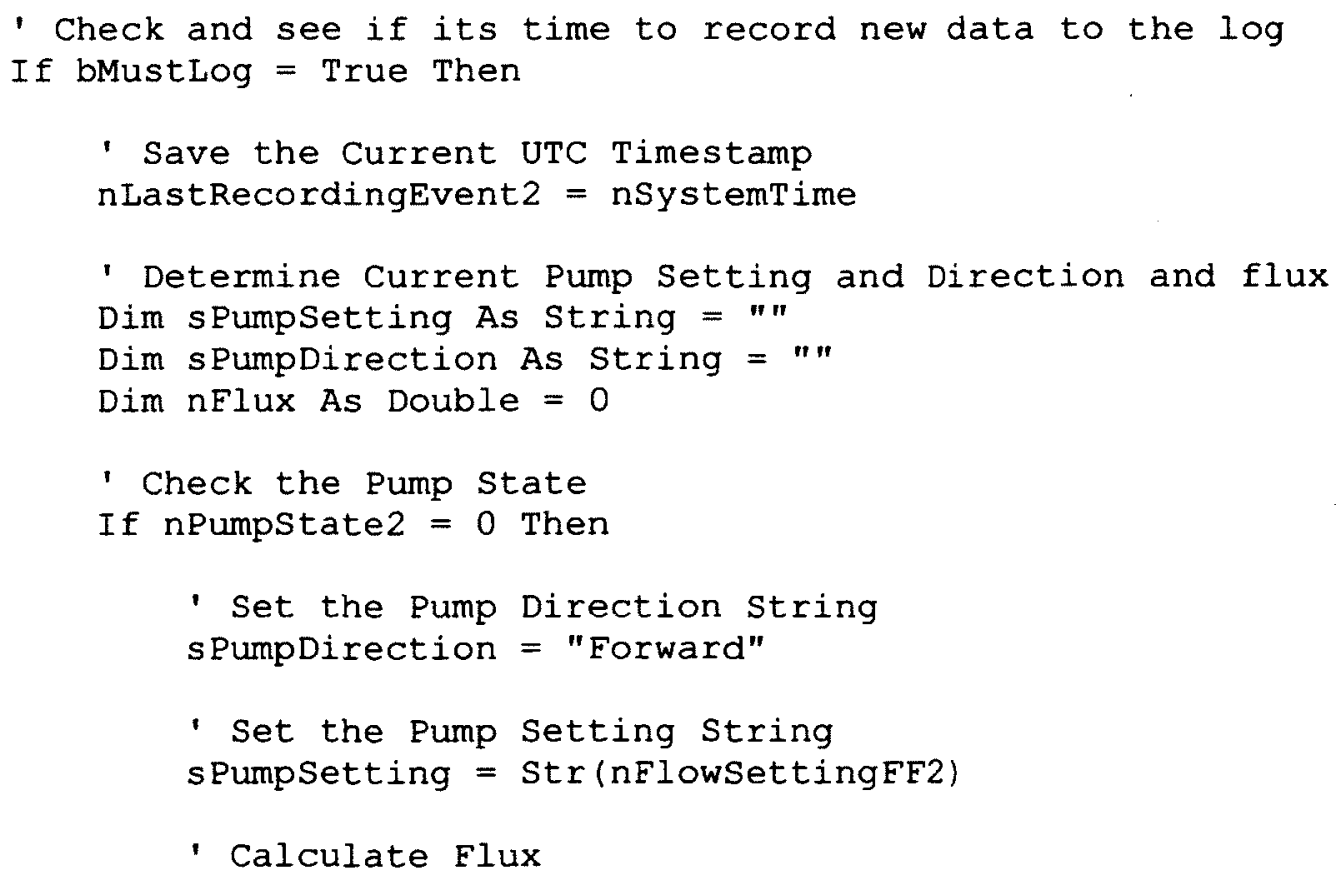


ElseIf nPumpstate $2=1$ Then

- Set the Pump Direction String

sPumpDirection = "Backward"

- set the Pump setting string sPumpsetting $=$ Str $(n F 1$ owsettingBF 2$)$

- Calculate Flux

$\mathrm{nFlux}=$ (nFlowSettingBF2 / nMembraneSurfaceArea2) *

$0.06 *-1$

ElseIf nPumpstate2 $=2$ Then

- Set the Pump Direction String

sPumpDirection = "Relax"

' Set the Pump setting string

s Pumpsetting $="-"$

- No Flux

nFlux $=0$

End If

' Check to see if we've hit the max allowed in the list If ListView2. Items.Count $=14$ Then

- Remove the oldest item from the list

ListView2 . I tems. RemoveAt (0)

End If

- Create a new string array to hold the recorded values

Dim slistItems(6) As String

Dim itm As ListViewItem

sListItems $(0)=$ Date. Now

sListItems $(1)=$ sPumpsetting

sListItems $(2)=$ sPumpDirection

slistItems $(3)=$ sFlowReading 2 ' handled by delegate

ReadAdamValue

sListItems $(4)=$ sPressureReading2 ' handled by

sListItems (5) = nFlux.Tostring ("F03")

'sListItems $(6)=$ "Room"

- Default For Now

itm $=$ New ListViewItem(sListItems)

ListView2.Items. Add (itm)

- Increment the number of

nLogEntries $2=$ nLogEntries $2+1$

- Open the file to write to and write the data 
Dim objStreamWriter As System.IO.StreamWriter

objstreamWriter $=$ New

System.IO.StreamWriter(sRecordingFilePathName2, True,

system. Text.Encoding. ASCII)

- Create the string to write to file

Dim sLine As String = sListItems $(0)+", "+$

Str(nUTCDeltaseconds) + "," + sListItems (4) + ", + sListItems (1) +

$", "+\operatorname{slistItems}(2)+", "+\operatorname{sListItems}(3)+", "+\operatorname{sListitems}(5)+$

", + "Room"

- Remove all spaces from the string

Dim sNewLine As String = ""

For $i$ As Integer $=0$ To sLine. Length -1

If sLine.Substring(i, 1$)<" ~ "$ Then End If

sNewLine $=$ sNewLine + sLine.Substring $(i, 1)$

Next

- Write the line to file

objStreamWriter.Writeline (sNewLine)

End If

objStreamWriter.Close()

End Sub

Private Sub Timer1 Tick (ByVal sender As System.object, ByVal e As System.EventArgs) Handles Timer1.Tick

- Get the current system Time

Dim nSystemTime As Long = Date.Now.ToFileTimeUtc()

' Update the Elapsed Time

Dim nUTCDelta As Long = nsystemTime - nstartTimeofRecording

Dim nUTCDeltaseconds As Long = nuTCDelta / nsecondsToUTC

Dim aDate As Date = Date. EromFileTimeUtc (nUTCDelta)

Dim nDays As Integer $=$ nuTCDeltaseconds $/(60 \star 60 \star 24)$

TextBoxTime.Text = "Elapsed Time: (" + nDays.Tostring("N0")

+ ") " + aDate.Tostring ("HH:mm:ss")

- Read the next value from the ADAM.

- Note: The Flow Meter Readings will be handled by the

- DataReceivedFlowMeter() Function.

ReadAdamValue()

- Process the two systems

Processsystem 1 (nsystemTime, nuTCDeltaseconds)

ProcessSystem2 (nsystemTime, nUTCDeltaseconds)

- Update live displays

TextBoxliveFlow. Text = sElowMeterDisplay

TextBoxLiveFlow2. Text $=$ sFlowReading2

TextBoxLivePressure. Text $=$ sPressureReading

TextBoxLivePressure2. Text = sPressureReading2 
End Sub

Private Sub DataReceivedFlowMeter (ByVal sender As Object, ByVal e As System.IO.Ports.SerialDataReceivedEventArgs) Handles

SerialPortFlowMeter. DataReceived

- Invoke Data Handler For Flow Meter Data

Me.BeginInvoke (New flowmeterDelegate (Addressof

handleIncomingDataflowMeter), New Object() (t)

End Sub

Public Delegate Sub flowmeterDelegate()

Public Sub handleIncomingDataFlowMeter()

- Check and see if the system is stopping

If bstopping = True Then

'Close Serial Port

CloseserialflowMeter()

Else

- Ensure the Flow Meter Serial Port is open

If SerialPortFlowMeter.Isopen = True Then

Buffer it

- Read the String Data off the Serial Port and

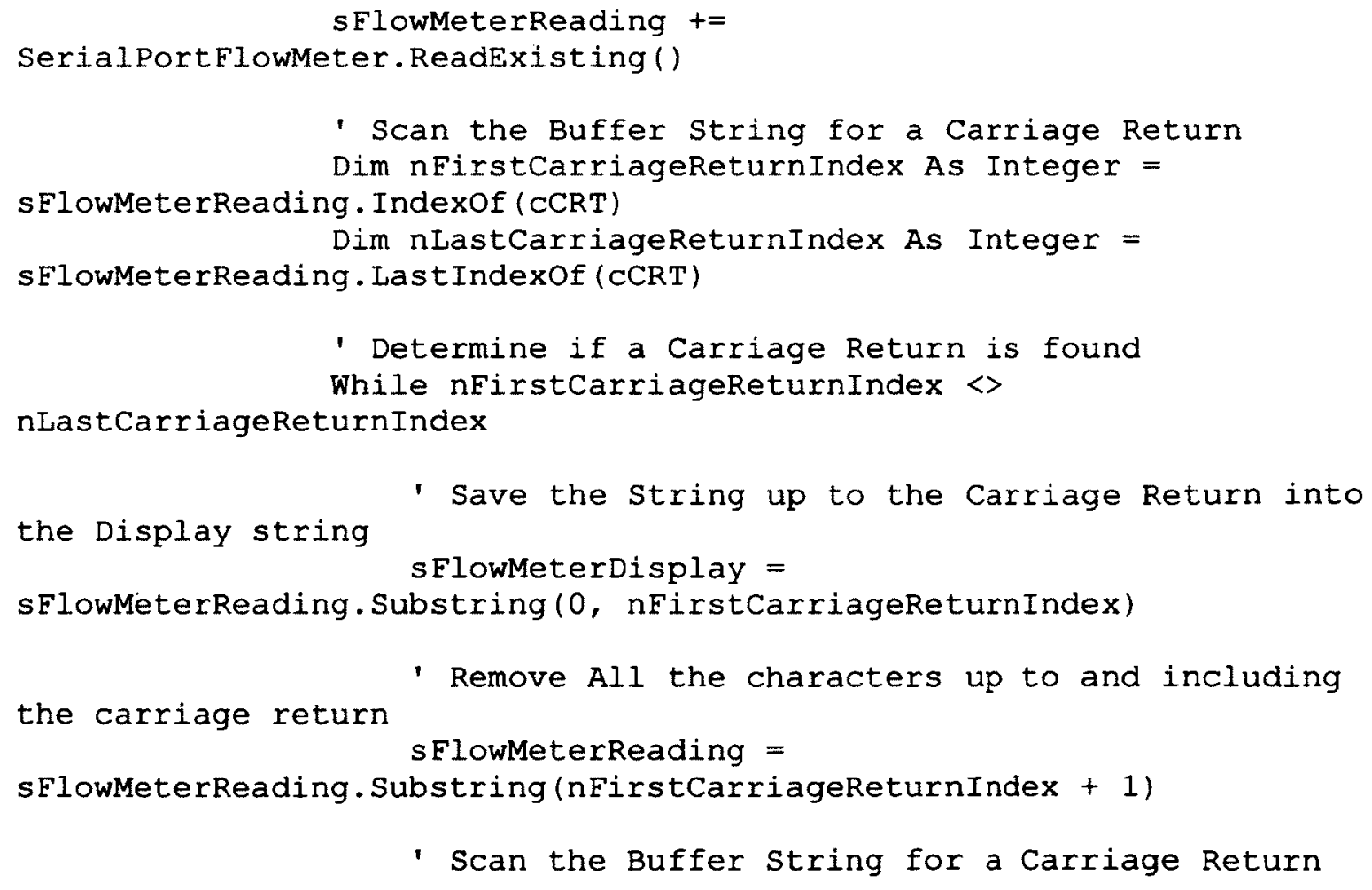




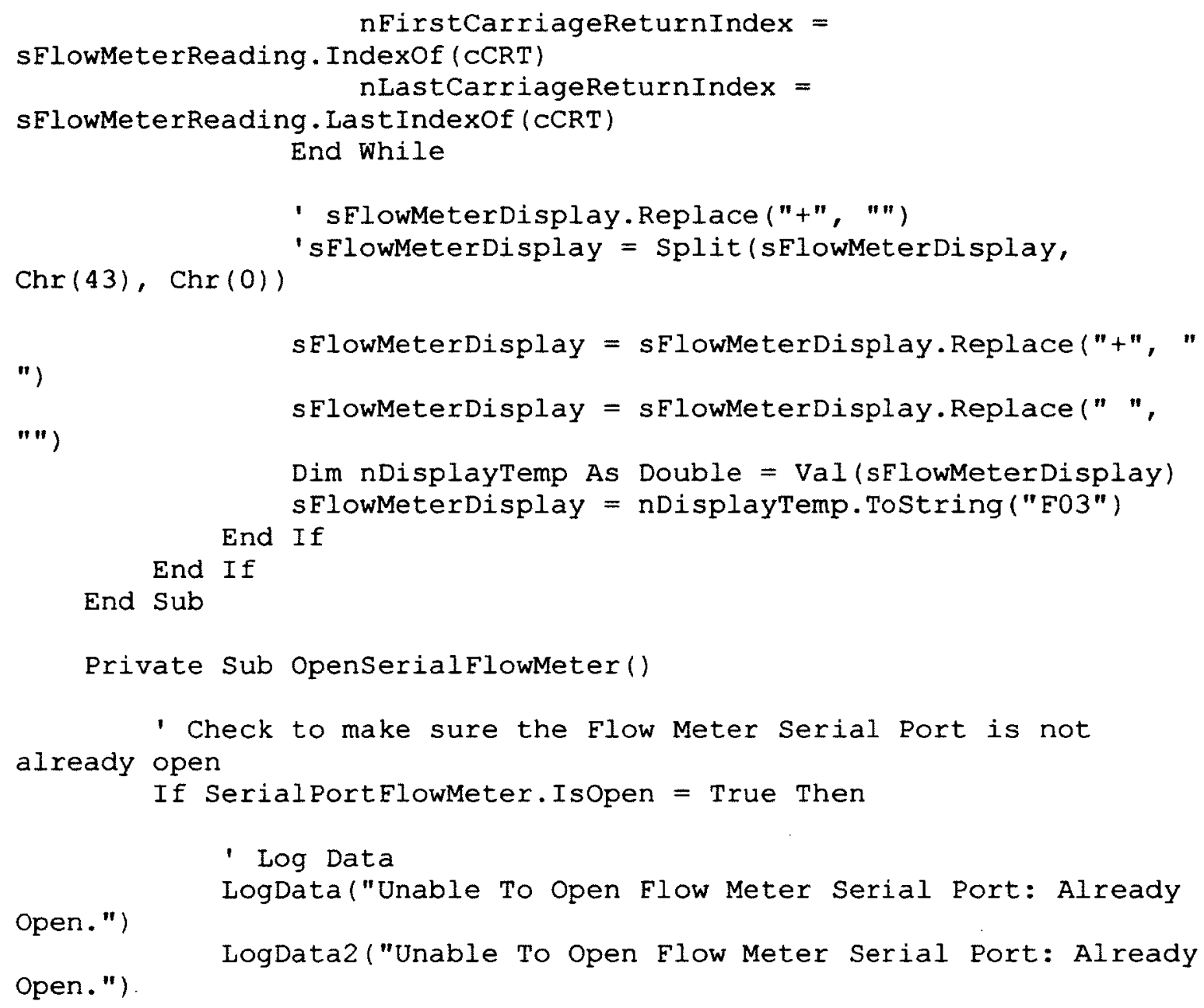




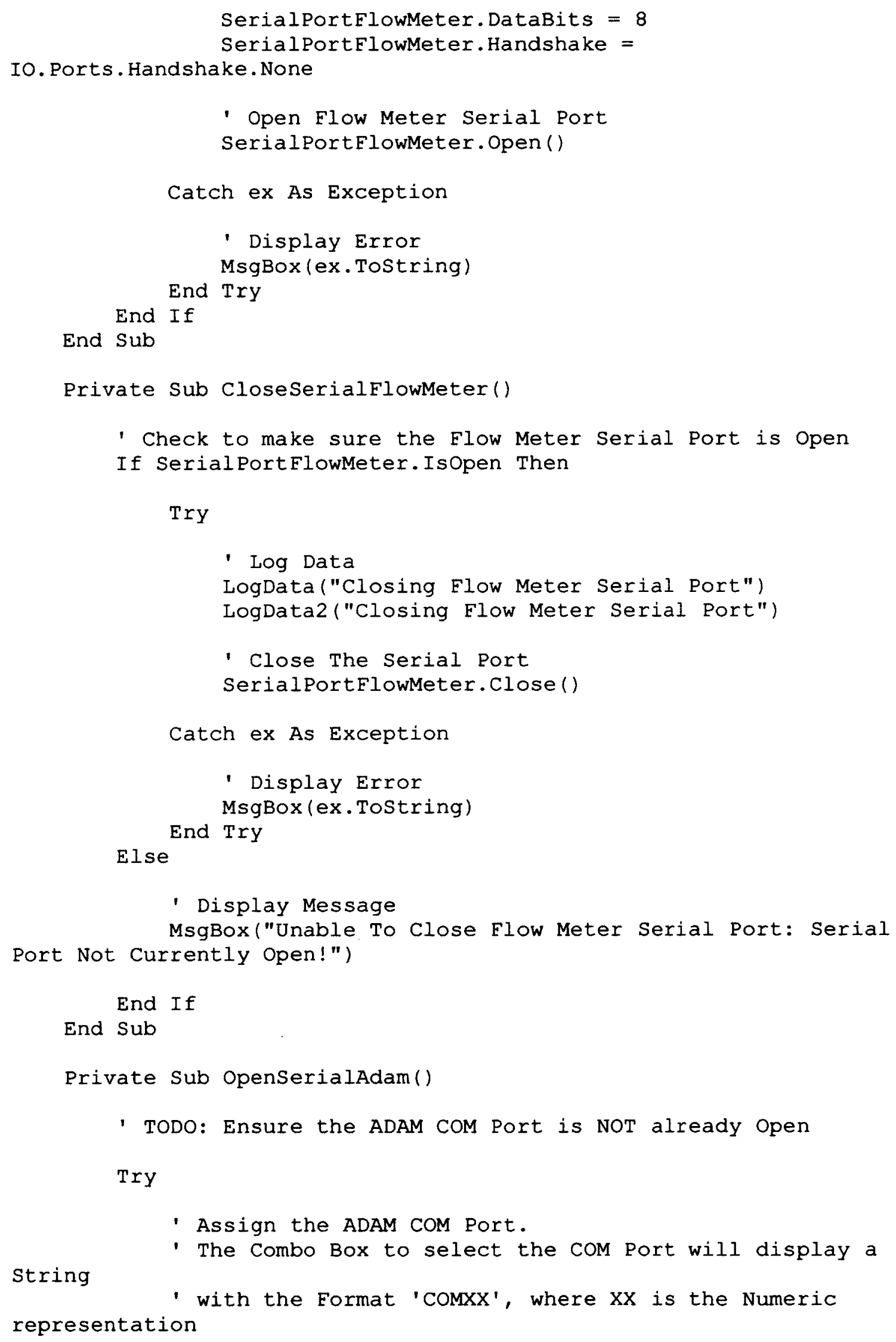




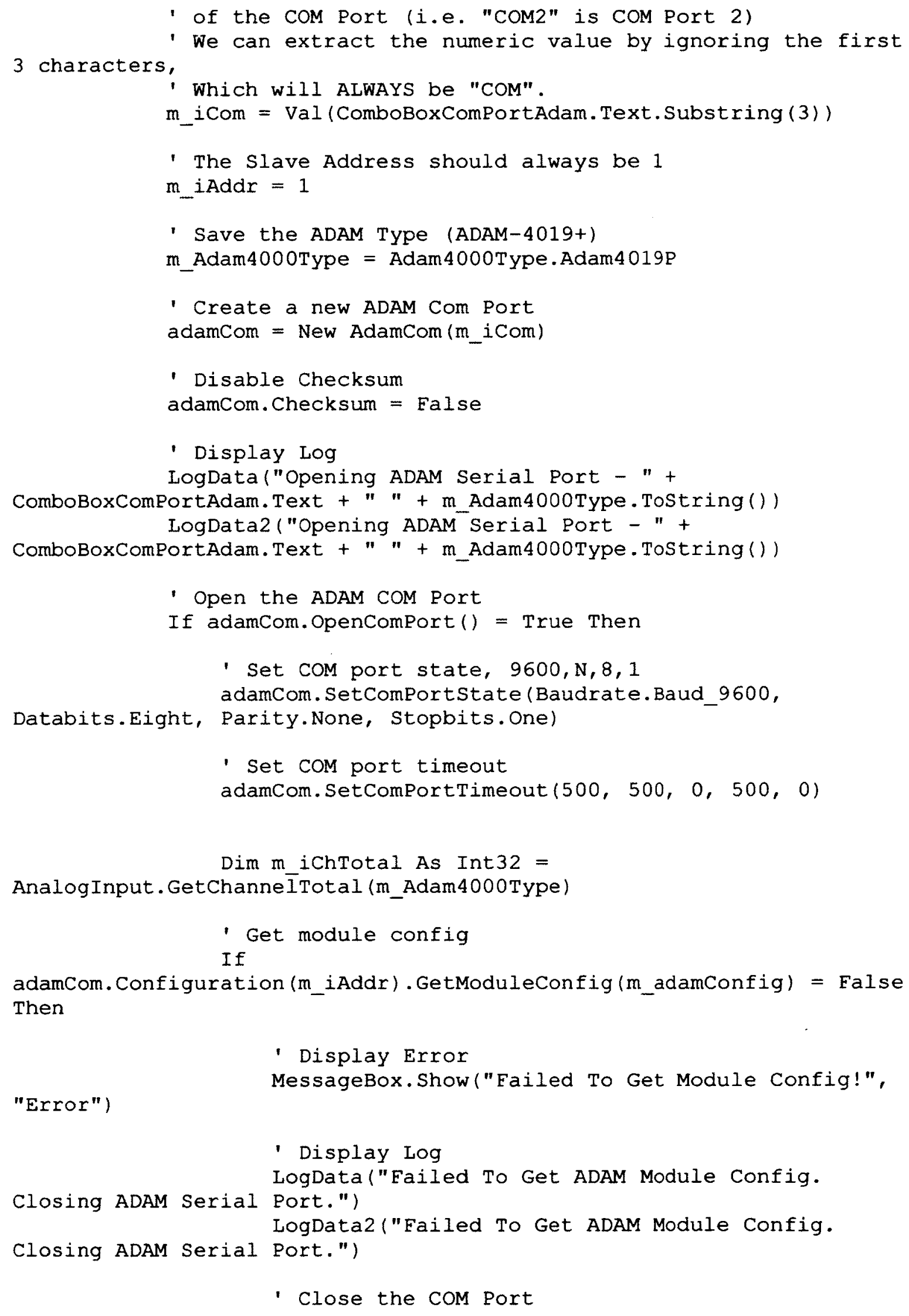




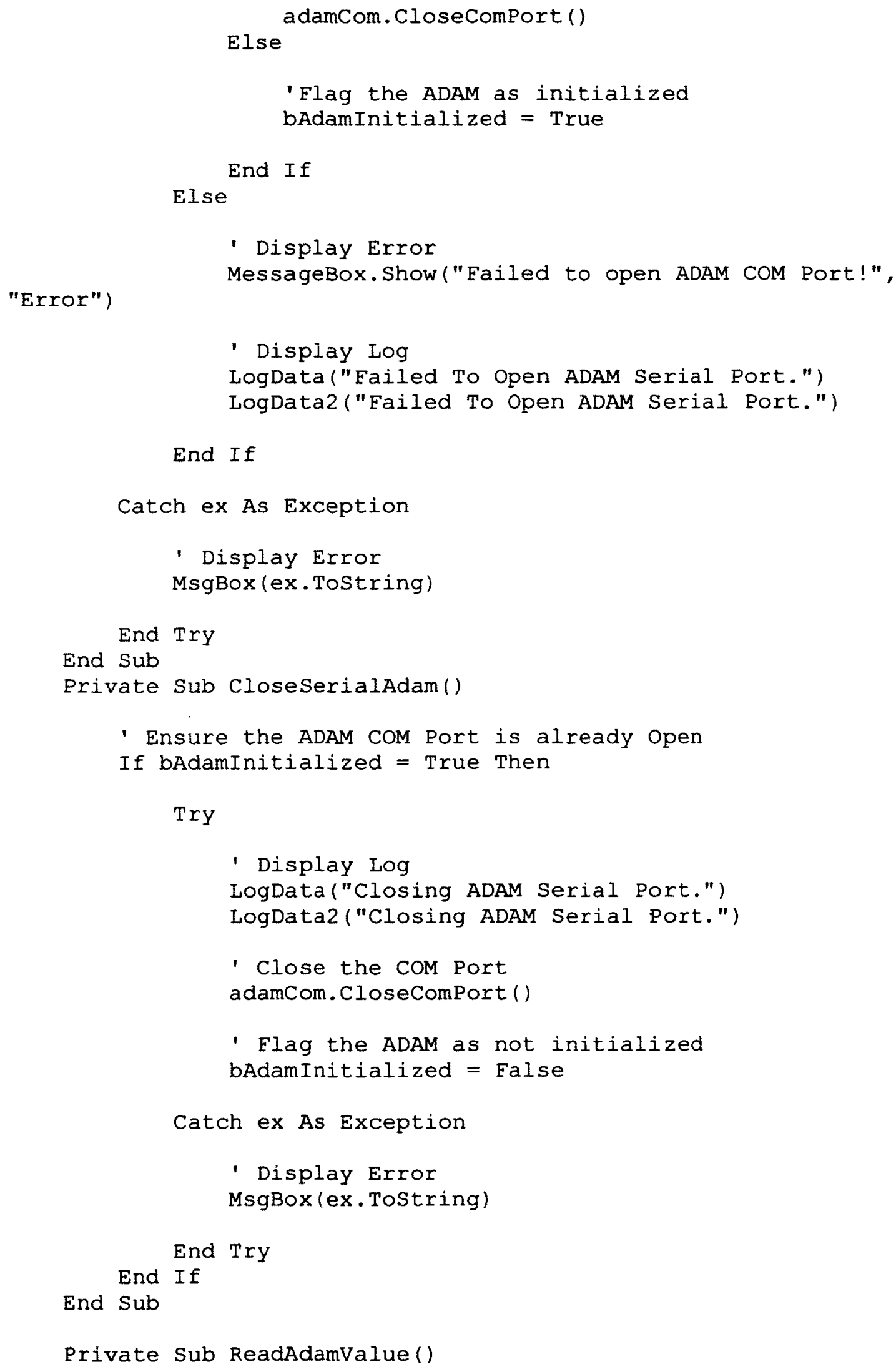


- Ensure the ADAM COM Port is already Open

If bAdamInitialized $=$ True Then

- Check the current data format of the ADAM

If m_adamConfig. Format =

Adam4000_DataFormat.TwosComplementHex Then

- Get the values as integers

Dim ivals As Int32()

$=$ True Then

If adamCom.AnalogInput (m_iAddr).GetValues(8, iVals)

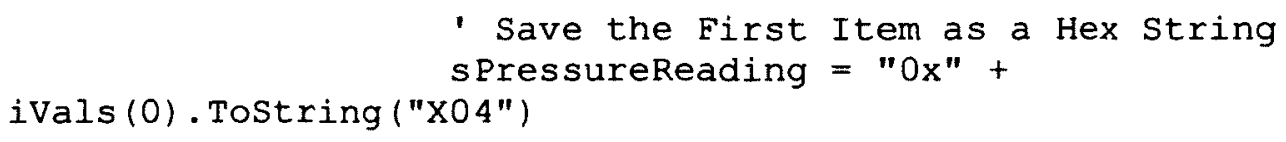

Else

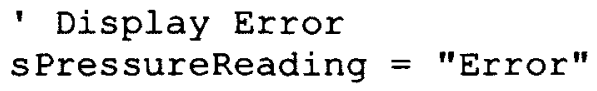

End If

Else

- Get the Values as Single Floating Point Values

Dim fVals As Single()

Dim status As Adam4000 Channelstatus()

If adamCom.Analog Input (m_iAddr).GetValues ( 8 , fVals,

status) $=$ True Then

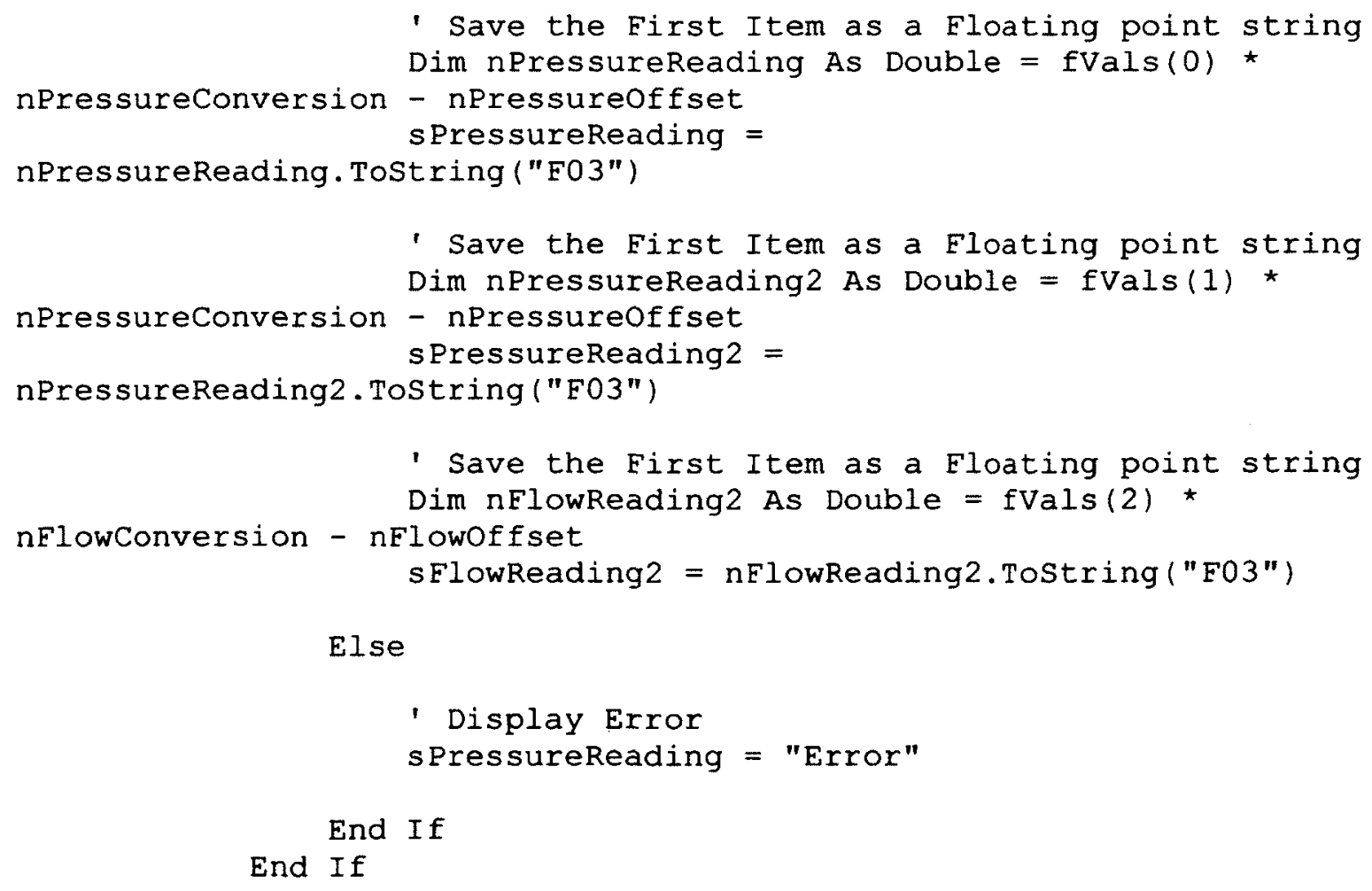

End If 
End If

End Sub

Private Sub LogData(ByVal sLog)

- Display Log

ListBoxLog. Items. Add (TimeofDay + " " + sLog)

- Select the last item in the list to scroll the list

ListBoxLog.SetSelected (ListBoxLog. Items.Count - 1, True)

End Sub

Private Sub LogData2 (ByVal sLog)

- Display Log

ListBoxLog2. Items. Add (TimeofDay + " $"+$ sLog)

- Select the last item in the list to scroll the list

ListBoxLog2. SetSelected (ListBoxLog2. Items.Count - 1, True)

End Sub

'Private Sub DataReceivedPump (ByVal sender As Object, ByVal e As System. Io.Ports.SerialDataReceivedEventArgs) Handles

SerialPortPump. DataReceived

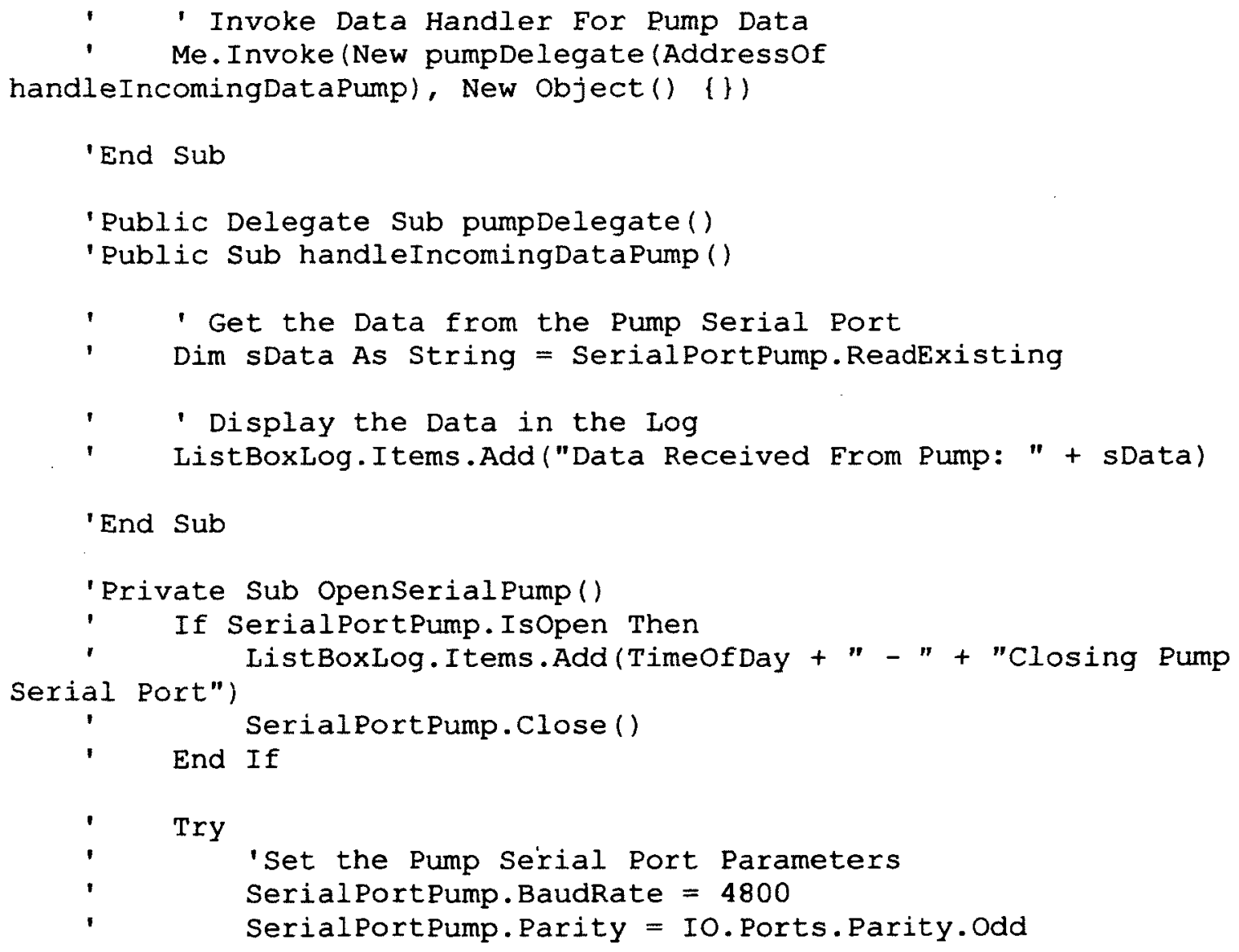




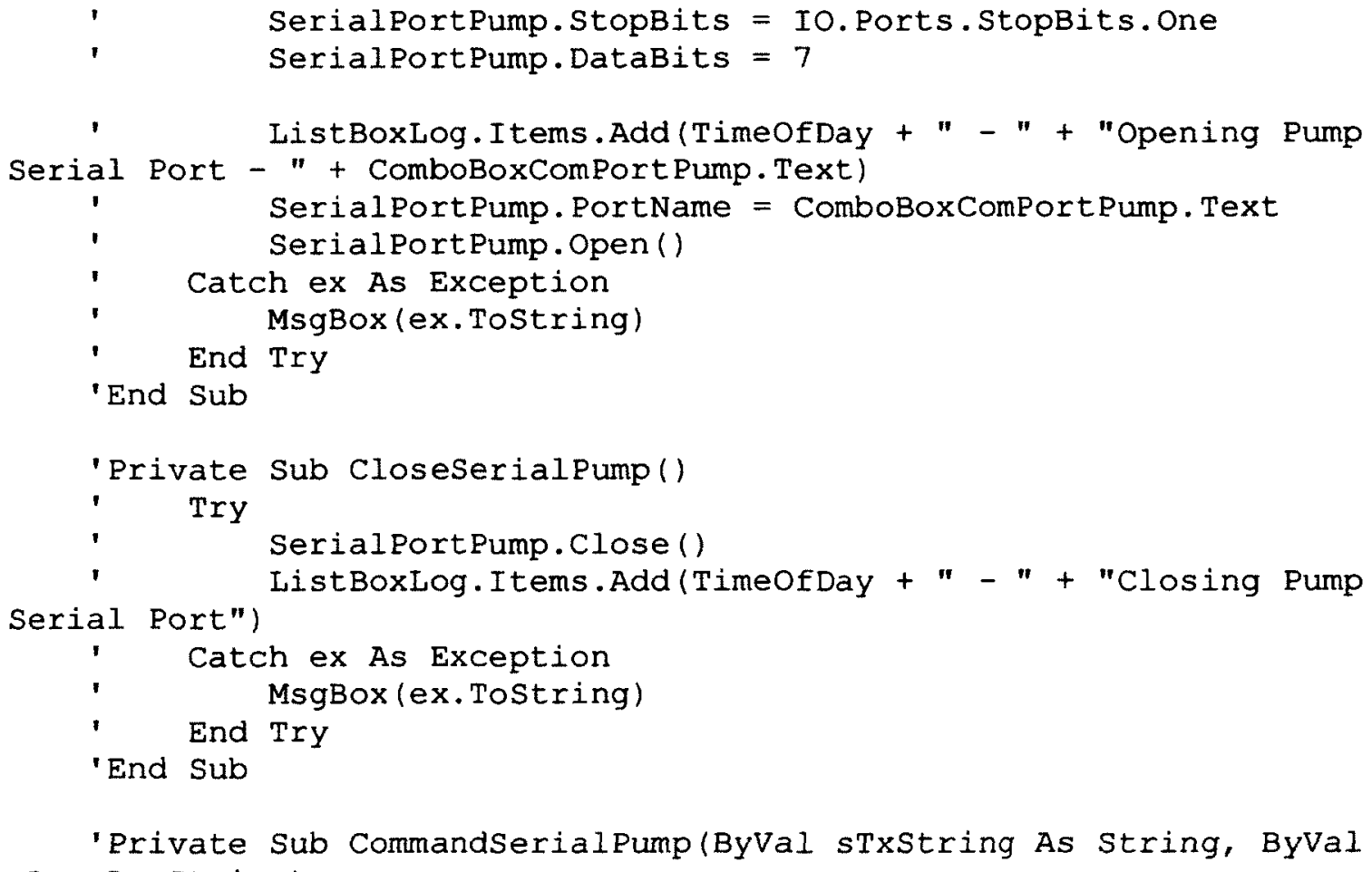

'Private Sub CommandSerialPump(ByVal sTxString As String, ByVal sLog As String)

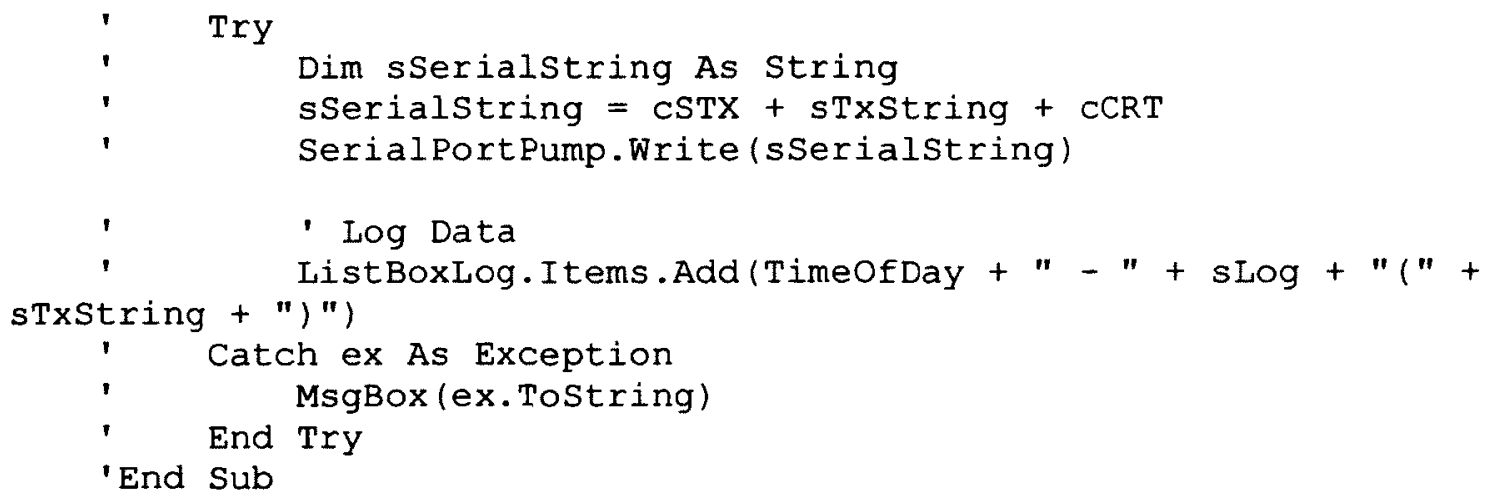

End Class 


\section{APPENDIX D - Wiring Diagram of Dual Experimental Setup}

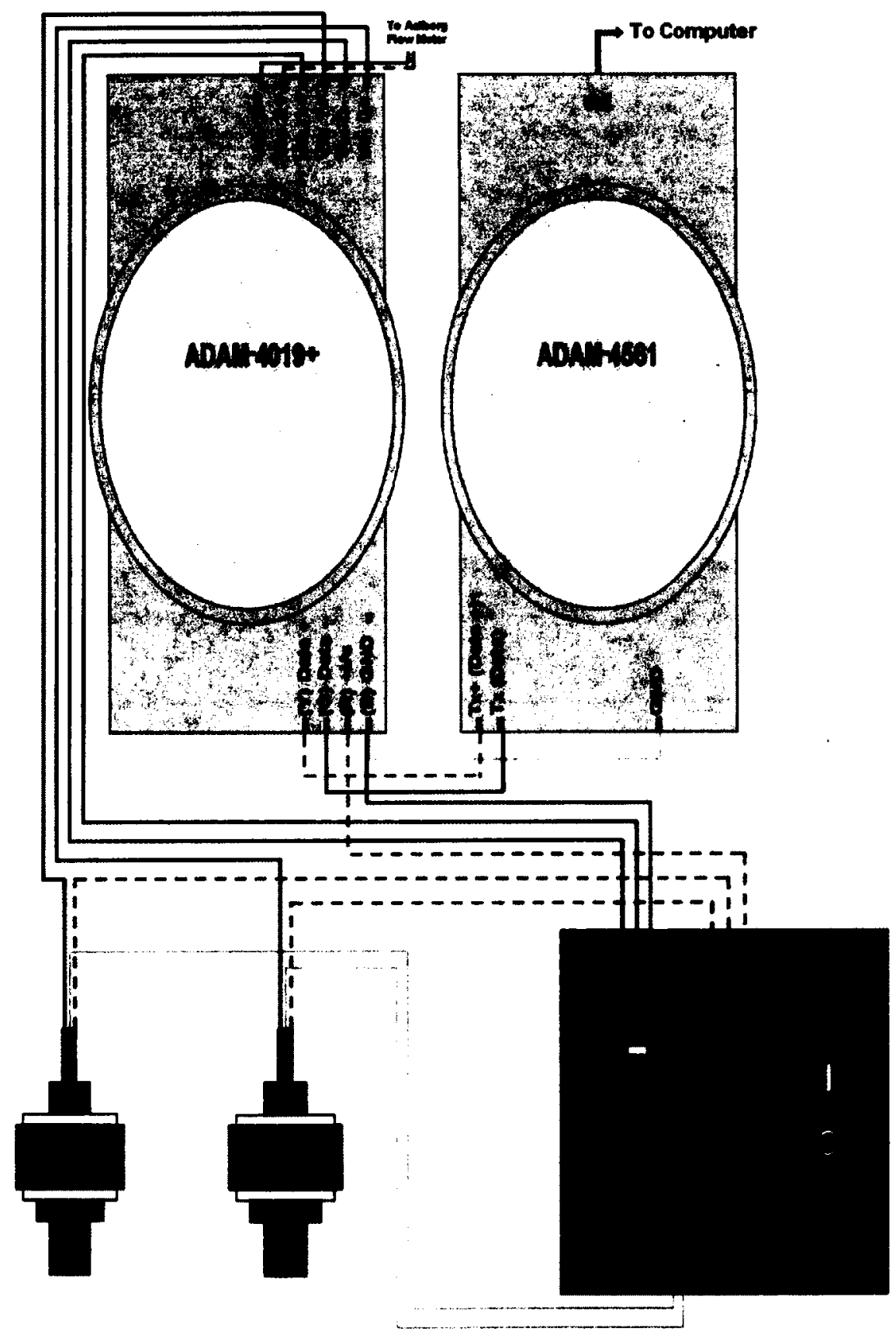




\section{APPENDIX E - Trouble Shooting for Operation Flux 2.0 and WinLIN}

\section{Operation Flux 2.0:}

For optimal performance of Operation Flux 2.0, it is recommended that the program be run in 'Debug' mode in Microsoft Visual Studio 10.0. The program may be accessed by opening Microsoft Visual Studio 10.0 available in the Start menu and proceeding with the following: File $>$ Open Project $>$ Operation Flux 2.0> Operation Flux (Solutions). Once the script appears on the screen, continue to Debug $>$ Start Debugging. A window with the Operation flux 2.0 interface will then appear at which time COM Port 12 and COM Port 5 must be assigned for the ADAM module and flow meter options respectively.

Once the COM ports are assigned, the program must be quickly tested to ensure communication with the program, ADAM module and flow meter. If pressure readings do not appear or if the error message "Fail to receive module configuration" appears, the following steps must be taken to resume connectivity with Operation flux and the Adam Module.

1. Check for Power in the ADAM modules. 
Operation Flux 2.0 may fail to receive the ADAM module configuration if the module is not powered up. The ADAM module comprises of the ADAM-4019 and ADAM-4561 modules. While the ADAM-4561 module is powered by the computer, the ADAM 4019-module is powered by an external power unit. Power to each module is denoted by a green light on the Module casing. If the light on the ADAM4561 is off, check to see if the USB connections on the module and computer are tight. If the light on the ADAM-4561 is off, check to see if the power unit is switched on. If the power unit is on but the module is still not powered, gently check the wiring into the module and power unit for any loose connections and tighten.

2. Update driver and assign COM Port number.

If both modules are powered but Operation flux 2.0 continues to "Fail to receive module configuration", it is very likely that the driver of the USB to Serial converter was automatically updated by Windows 7 thus changing the assigned COM port number (COM 12) to an arbitrary COM port number. Such an update of the driver usually occurs when a connection to the internet is initiated and any internet connection should be avoided. A specific driver is required for the USB to serial converter connecting the ADAM module to the computer. The ADAM 4561-4562 USB to serial driver can be found as follows: Desktop> Programs> ADAM_45614562>98_2k-XP-20026. To install this driver, the required COM port for the ADAM module must first be identified. 
Begin the identification process by clicking the Control Panel available on the Start menu. Proceed to Hardware and Sound $>$ Devices and Printers $>$ Device Manager $>$ Ports (COMS and LPT). A number of COM ports will be listed under Ports (COMS and LPT). The COM port corresponding to the USB to Serial converter connecting the ADAM module to the computer may be identified as the COM port other than COM port 5 and COM port 11. To change the port number of the identified COM port to COM port 12, under Ports (COMS and LPT) right click the identified port and choose Properties $>$ Port Settings $>$ Advanced $>$ COM port number. The COM port number may now be changed to COM port 12 .

Once identified, the proper driver may be installed by right clicking on the COM port under Ports (COMS and LPT) and choosing Properties> Driver> Update Driver> Browse my computer $>$ Desktop $>$ Programs $>$ ADAM_4561-4562 $>$ 98_2k-XP20026.

Note: Three sets of data readings are obtained through the ADAM module; the pressure readings from pressure transducers used in Setup 1 and 2 as well as the air flow rate for Setup 2. Thus if Operation Flux 2.0 fails to connect to the ADAM Module, the three aforementioned sets of data will not appear in the assigned columns nor will they be recorded. 


\section{WinLIN:}

On connecting to the internet or on restarting the computer, the driver of the USB to Serial converter might undergo an automatic update by Windows 7 during which, the assigned COM port number (COM 11) might change. As a result, the program WinLIN will fail to connect to the digital pumps and fail to assign the order of the pumps (P1 and P2). If such an event occurs the following steps must be taken.

\section{Identify the proper COM port \#:}

Begin by clicking the Control Panel available on the Start menu. Proceed to Hardware and Sound $>$ Devices and Printers $>$ Device Manager $>$ Ports (COMS and LPT). A number of COM ports will be listed under Ports (COMS and LPT). To identify the COM port corresponding to the USB to Serial converter connecting the digital pumps to the computer, test the COM ports manually by sequentially assigning each COM port in WinLIN (Settings> COM ports) and running a test run for Pump 1. The required COM port is identified when no "Failure to Connect/Assign" message is observed and the pump drive starts to rotate.

\section{Changing the COM port \#:}


Once the proper COM port is identified, the new COM port number may be left as is and noted for further use or, the COM port number may be changed to COM 11 for common practice. To change the COM port number, identify the COM port listed under Ports (COMS and LPT). Right click the identified port and choose Properties $>$ Port Settings $>$ Advanced $>$ COM port number. The COM port number may now be changed to COM port 11 .

Note: The driver used for the USB to Serial Converter for the digital pumps should be listed as 'Prolific USB to Serial'. If this is not the case, the driver maybe updated by right clicking on the required COM port and choosing Properties $>$ Driver> Update Driver> Browse my computer. The required driver maybe found in the folder named 'Prolific Driver' on the Desktop. 


\section{APPENDIX F - Simulated Test Water Ingredients/ Organic}

Component Calibration Curve

\begin{tabular}{|l|c|c|c|}
\hline Constituent & $1 \mathrm{~L}$ & $42 \mathrm{~L}$ & $50 \mathrm{~L}$ \\
\hline Humic acid $(\mathrm{mg})$ & 8.95 & 375.90 & 447.50 \\
\hline Cellulose $(\mathrm{mg})$ & 7.14 & 299.88 & 357.00 \\
\hline Kaolin Clay $(\mathrm{mg})$ & 41.00 & 1600.00 & 1650.00 \\
\hline $\mathrm{NaHCO}_{3}(\mathrm{mg})$ & 67.01 & 2814.42 & 3350.50 \\
\hline $\mathrm{CaSO}_{4}(\mathrm{mg})$ & 129.13 & 5423.46 & 6456.50 \\
\hline
\end{tabular}

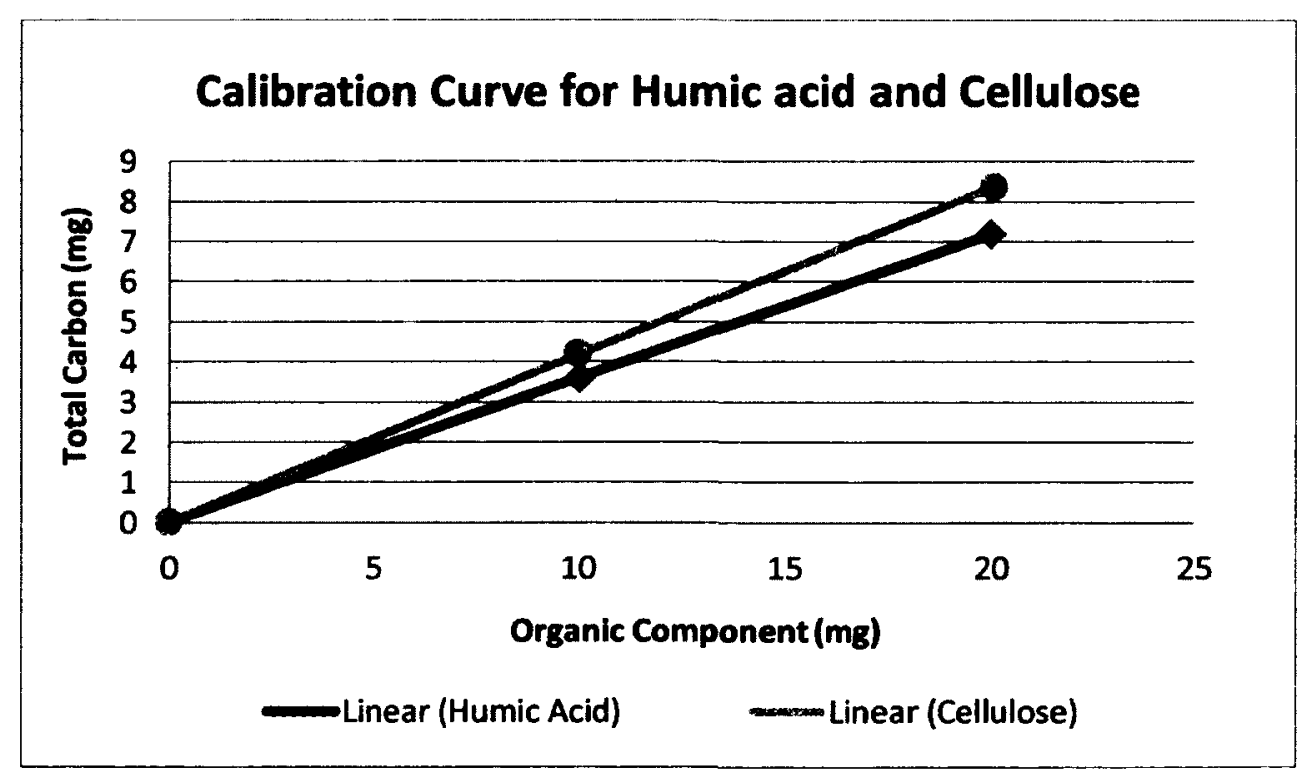




\section{APPENDIX G - Macro code for filtering backwash and}

\section{relaxation data points from TMP curves}

Sub BWRfilt()

' BWRfilt Macro

' Authors: Kelly Alder (B.Eng, M.A.Sc) and Nigel De Souza (B.Eng)

' Keyboard Shortcut: Ctrl+n

,

' Create a variable to hold the column id

Dim sColumn As String

Dim sColumnHeader As String

' Create a new variable to hold the cell value

Dim nValue As Double

' Create a new variable to hold percent difference

Dim nPercentDiff As Double

' Create a new variable to hold previos value

Dim nPrevValue As Double

' Create a string representation for the Column Id

$\mathrm{sColumn}=\mathrm{Chr}($ ActiveCell. Column +64$)+" 1 "$

' Go to the top of the selected column

Range(sColumn).Select

' Get the text of the column header

sColumnHeader $=$ ActiveCell. Text

- Trim all leading and trailing whitespaces

sColumnHeader $=$ Trim $($ sColumnHeader $)$

' Make the Column HeEader uppercase

' for case-insensitive comparison

sColumnHeader $=$ UCase $($ sColumnHeader $)$

' Make sure we're looking at a Pressure Reasing Column

If sColumnHeader = "PRESSURE_READING" Then 
' Move the selected cell one row down ActiveCell.Offset(2,0).Select

' Check that we haven't reached the end by checking that it's not blank While (ActiveCell.Text $\diamond$ "')

' Get the cell value and previous cell value

nValue $=$ ActiveCell. Value

' Check to see if a row is positive

If nValue $>0$ Then

' Delete the positive row

Rows(ActiveCell.Row).EntireRow.Delete

Else

' Get the value of the previous cell

nPrevValue $=$ ActiveCell.Offset $(-1,0)$. Value

' Check to see if the cell value is greater than

' the previous value

If $n$ Value $>$ nPrevValue Then

'Calculate percent difference

$\mathrm{nPercentDiff}=(\mathrm{nPrevValue}-\mathrm{nValue}) / \mathrm{nPrevV}$ alue $* 100$

' Check to see if percent difference is larger that our tolerance If $n$ PercentDiff $>5$ Then

' Delete this row, we dont want it

Rows(ActiveCell.Row).EntireRow.Delete

Else

' Move the selected cell one row down

ActiveCell.Offset $(1,0)$.Select

End If

Else

' Move the selected cell one row down

ActiveCell.Offset $(1,0)$.Select

End If

End If

Wend

Else 


\author{
' Display Warning \\ MsgBox ("Macro Must Be Run On a PRESSURE_READING Column!") \\ End If \\ End Sub
}


APPENDIX $\mathrm{H}$ - ANOVA MODEL for a $\mathbf{2}^{\mathbf{4}}$ factorial design

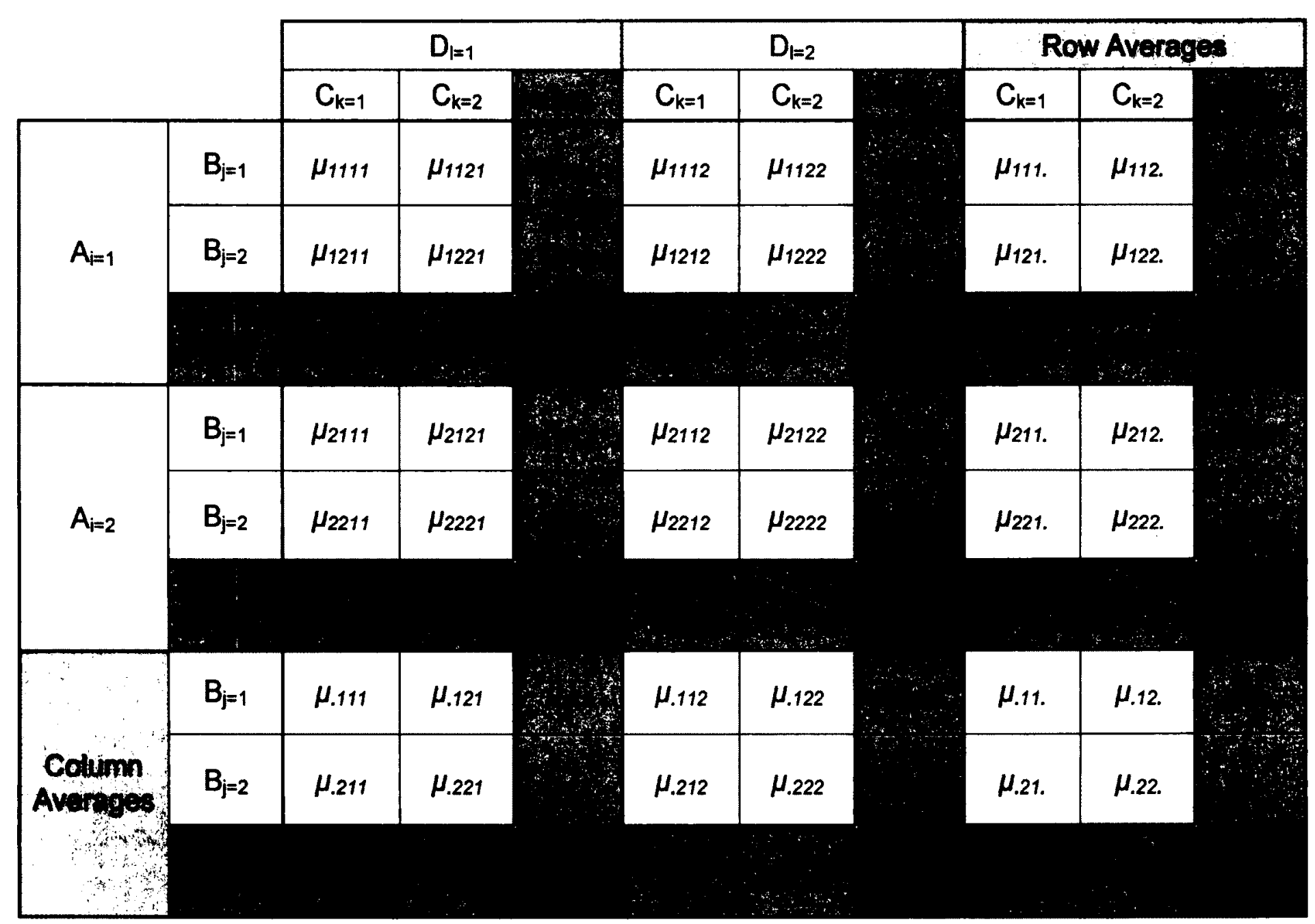


Main Effects:

$$
\begin{aligned}
& \alpha_{i}=\mu_{i . . .}-\mu_{. . .} \\
& \beta_{j}=\mu_{. j .}-\mu_{\ldots .} \\
& \gamma_{k}=\mu_{. k .}-\mu_{\ldots .} \\
& \delta_{j}=\mu_{. . . l}-\mu_{\ldots . .}
\end{aligned}
$$

Two factor interactions:

$$
\begin{aligned}
& \alpha \beta_{i j}=\mu_{i j . .}-\left(\alpha_{i}+\beta_{j}+\mu_{. . .}\right) \\
& \alpha \gamma_{i j}=\mu_{i . k .}-\left(\alpha_{i}+\gamma_{k}+\mu_{\ldots . .}\right) \\
& \alpha \delta_{i j}=\mu_{i . l}-\left(\alpha_{i}+\delta_{l}+\mu_{\ldots . . .}\right) \\
& \beta \gamma_{j k}=\mu_{. j k .}-\left(\beta_{j}+\gamma_{k}+\mu_{. . . .}\right) \\
& \beta \delta_{j l}=\mu_{. j . l}-\left(\beta_{j}+\delta_{l}+\mu_{\ldots . .}\right) \\
& \gamma \delta_{k l}=\mu_{. . k l}-\left(\gamma_{k}+\delta_{l}+\mu_{. . .}\right)
\end{aligned}
$$

Three factor interactions:

$$
\begin{aligned}
& \alpha \beta \gamma_{i j k}=\mu_{i j k .}-\left(\alpha_{i}+\beta_{j}+\gamma_{k}+\alpha \beta_{i j}+\alpha \gamma_{i k}+\beta \gamma_{j k}+\mu_{\ldots . .}\right) \\
& \alpha \beta \delta_{i j l}=\mu_{i j . l}-\left(\alpha_{i}+\beta_{j}+\delta_{l}+\alpha \beta_{i j}+\alpha \delta_{i l}+\beta \delta_{j l}+\mu_{\ldots . .}\right) \\
& \alpha \gamma \delta_{i k l}=\mu_{i . k l}-\left(\alpha_{i}+\gamma_{k}+\delta_{l}+\alpha \gamma_{i k}+\alpha \delta_{i l}+\gamma \delta_{k l}+\mu_{\ldots . .}\right) \\
& \beta \gamma \delta_{j k l}=\mu_{. j k l}-\left(\beta_{j}+\gamma_{k}+\delta_{l}+\beta \gamma_{j k}+\beta \delta_{j l}+\gamma \delta_{k l}+\mu_{\ldots . . .}\right)
\end{aligned}
$$

Four factor interactions:

$\alpha \beta \gamma \delta_{i j k l}=\mu_{i j k l}-\left(\alpha_{i}+\beta_{j}+\gamma_{k}+\delta_{l}+\alpha \beta_{i j}+\alpha \gamma_{i k}+\alpha \delta_{i l}+\beta \gamma_{j k}+\beta \delta_{j l}+\gamma \delta_{k l}+\right.$ $\left.\alpha \beta \gamma_{i j k}+\alpha \beta \delta_{i j l}+\alpha \gamma \delta_{i k l}+\beta \gamma \delta_{j k l}+\mu_{\ldots . .}\right)$ 


\section{APPENDIX I - Similarities between ANOVA parameters and regression coefficients}

\begin{tabular}{|c|c|}
\hline$\mu \ldots$ & $\boldsymbol{\beta}_{0}$ \\
\hline$\alpha_{2}$ & $\boldsymbol{\beta}_{1}$ \\
\hline $\boldsymbol{\beta}_{2}$ & $\boldsymbol{\beta}_{2}$ \\
\hline$\gamma_{2}$ & $\boldsymbol{\beta}_{\mathbf{3}}$ \\
\hline$\delta_{2}$ & $\boldsymbol{\beta}_{4}$ \\
\hline Wh & 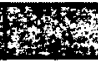 \\
\hline$\alpha \beta_{22}$ & $\beta_{12}$ \\
\hline$a \gamma_{22}$ & $\beta_{13}$ \\
\hline$\alpha \delta_{22}$ & $\beta_{14}$ \\
\hline$\beta \gamma_{22}$ & $\beta_{23}$ \\
\hline$\beta \delta_{22}$ & $\beta_{24}$ \\
\hline$\gamma \bar{\delta}_{22}$ & $\boldsymbol{\beta}_{34}$ \\
\hline$\alpha \beta \gamma_{222}$ & $\boldsymbol{\beta}_{123}$ \\
\hline$\alpha \beta \delta_{222}$ & $\boldsymbol{\beta}_{124}$ \\
\hline$\alpha \gamma \delta_{222}$ & $\boldsymbol{\beta}_{134}$ \\
\hline$\beta \gamma \delta_{222}$ & $\boldsymbol{\beta}_{234}$ \\
\hline$\alpha \beta \gamma \delta_{2222}$ & $\beta_{1234}$ \\
\hline
\end{tabular}




\section{APPENDIX J - Water quality data from Preliminary Study}

\section{Phase A}

\begin{tabular}{|c|c|c|c|}
\hline Sample & COD (mg/L) & $\mathrm{pH}$ & Turbidity \\
\hline \multicolumn{4}{|c|}{ Experiment 1 - duration 5 mins. Interval 2 hrs } \\
\hline Initial Feed & 12 & 7.43 & 56 \\
\hline Initial Permeate & 1.5 & 7.08 & 0.05 \\
\hline Feed in feed tank (12 hrs) & 8 & 7.36 & 31.24 \\
\hline Feed in reactor tank ( $12 \mathrm{hrs})$ & 51.5 & 7.3 & 188.3 \\
\hline Permeate (12 hrs) & 2 & 7.18 & 0.53 \\
\hline \multicolumn{4}{|c|}{ Experiment 2 - duration 15 mins. Interval 2 hrs } \\
\hline Initial Feed & 17 & 7.86 & 73.4 \\
\hline Initial Permeate & 2 & 7.6 & 0.21 \\
\hline Feed in feed tank (20 hrs) & 7 & 7.52 & 30.51 \\
\hline Feed in reactor tank (20 hrs) & 25 & 7.46 & 64.2 \\
\hline Permeate (12 hrs) & & 7.51 & 0.13 \\
\hline \multicolumn{4}{|c|}{ Experiment 3 - duration 5 mins. Interval 4 hrs } \\
\hline Initial Feed & 15 & 7.53 & 65.29 \\
\hline Initial Permeate & 2 & 7.34 & 0.04 \\
\hline Feed in feed tank (16 hrs) & 16 & 7.17 & 40.15 \\
\hline Feed in reactor tank (16 hrs) & 67 & 7.38 & 203.7 \\
\hline Permeate (12 hrs) & & 7.36 & 0.07 \\
\hline \multicolumn{4}{|c|}{ Experiment 4 - duration 15 mins. Interval 4 hrs } \\
\hline Initial Feed & 24 & 8.06 & 56.15 \\
\hline Initial Permeate & 0 & 7.3 & 0.04 \\
\hline Feed in feed tank ( $16 \mathrm{hrs}$ ) & & 8 & 33.57 \\
\hline Feed in reactor tank ( $16 \mathrm{hrs})$ & & 8.01 & 85.87 \\
\hline Permeate (16 hrs) & & 7.35 & 0.01 \\
\hline
\end{tabular}




\section{APPENDIX K - Water quality data from Preliminary Study}

Phase B

Turbidity (NTU):

\begin{tabular}{|c|c|c|c|c|c|c|c|c|c|c|}
\hline & \multirow[b]{2}{*}{ Base } & \multirow[b]{2}{*}{ 12D5 } & \multirow[b]{2}{*}{ 12D15 } & \multirow[b]{2}{*}{ 14D5 } & \multirow[b]{2}{*}{ |4D15 } & \multicolumn{5}{|c|}{ Continuous flow } \\
\hline & & & & & & Base & 12D5 & 12D15 & 14D5 & 14D15 \\
\hline $\begin{array}{l}\text { Feed } \\
\text { (Initial) }\end{array}$ & 26.9 & 29.14 & 26.84 & 29.66 & 30.17 & 29.46 & 30.23 & 30.92 & 30.81 & 26.47 \\
\hline $\begin{array}{l}\text { Feed } \\
\text { (12 hrs) }\end{array}$ & 18.96 & 18.16 & 17.14 & 16.16 & 22.07 & 28.42 & 27.18 & 28.51 & 24.51 & 22.24 \\
\hline $\begin{array}{l}\text { Feed } \\
\text { (24 hrs) }\end{array}$ & 11.21 & 8.72 & 9.82 & 10.15 & 10.03 & 19.61 & 26.04 & 27.31 & 28.21 & 23.33 \\
\hline $\begin{array}{l}\text { Reactor } \\
\text { (Initial) }\end{array}$ & 26.9 & 29.14 & 26.84 & 29.66 & 30.17 & 29.46 & 30.23 & 30.92 & 30.81 & 26.47 \\
\hline $\begin{array}{l}\text { Reactor } \\
\text { (12 hrs) }\end{array}$ & 62.19 & 117.6 & 113.7 & 101.6 & 155.2 & 46.07 & 82.92 & 61.72 & 71.04 & 75.66 \\
\hline $\begin{array}{l}\text { Reactor } \\
\text { (24 hrs) }\end{array}$ & 44.94 & 169.5 & 155.4 & 125.2 & 188.2 & 37.01 & 65.39 & 62.87 & 68.21 & 70.6 \\
\hline $\begin{array}{l}\text { Permeate } \\
\text { (Initial) }\end{array}$ & 0.19 & 0.01 & 0.05 & 0.15 & 0.2 & 0.11 & 0.15 & 0.18 & 0.13 & 0.07 \\
\hline $\begin{array}{l}\text { Permeate } \\
\text { (12 hrs) }\end{array}$ & 0.12 & 0.01 & 0.13 & 0.1 & 0.08 & 0.1 & 0.04 & 0.17 & 0.01 & 0.1 \\
\hline $\begin{array}{l}\text { Permeate } \\
\text { (24 hrs) }\end{array}$ & 0.18 & 0.01 & 0.06 & 0.01 & 0.11 & 0.05 & 0.1 & 0.01 & 0.01 & 0.02 \\
\hline
\end{tabular}


COD (mg/L COD):

\begin{tabular}{|c|c|c|c|c|c|c|c|c|c|c|}
\hline & \multirow{2}{*}{ Base } & \multirow[b]{2}{*}{ 12D5 } & \multirow[b]{2}{*}{ 12D15 } & \multirow[b]{2}{*}{ 14D5 } & \multirow[b]{2}{*}{ 14D15 } & \multicolumn{5}{|c|}{ Continuous flow } \\
\hline & & & & & & Base & 1205 & 12D15 & I4D5 & 14D15 \\
\hline $\begin{array}{l}\text { Feed } \\
\text { (Initial) }\end{array}$ & 13.5 & 15 & 13 & 9.5 & 9.5 & 17 & 13.5 & 16 & 20 & 14 \\
\hline $\begin{array}{l}\text { Feed } \\
\text { (12 hrs) }\end{array}$ & 8 & 8.5 & 6.5 & 5 & 9 & 15.5 & 11.5 & 14.5 & 13.5 & 11.5 \\
\hline $\begin{array}{l}\text { Feed } \\
\text { (24 hrs) }\end{array}$ & 4 & 8 & - & 3 & 5 & 12.7 & 16 & 12 & 16.5 & 7.5 \\
\hline $\begin{array}{l}\text { Reactor } \\
\text { (Initial) }\end{array}$ & 13.5 & 15 & 13 & 9.5 & 9.5 & 17 & 13.5 & 16 & 20 & 14 \\
\hline $\begin{array}{l}\text { Reactor } \\
\text { (12 hrs) }\end{array}$ & 32 & 55 & 42 & 44 & 56 & 19.5 & 33.5 & 25.5 & 31.5 & 36.5 \\
\hline $\begin{array}{l}\text { Reactor } \\
\text { (24 hrs) }\end{array}$ & 13 & 62.5 & 53 & 46.5 & 68.5 & 19 & 29.5 & 32.5 & 35.5 & 27.5 \\
\hline $\begin{array}{l}\text { Permeate } \\
\text { (Initial) }\end{array}$ & 14 & 2 & 3 & 3.35 & 5.25 & 3.45 & 17.45 & 3.25 & 2.9 & 3.8 \\
\hline $\begin{array}{l}\text { Permeate } \\
\text { (12 hrs) }\end{array}$ & 2.6 & 4 & 2.95 & 2.6 & 4.2 & 4.1 & 3.3 & 2.65 & 3.15 & 4.3 \\
\hline $\begin{array}{l}\text { Permeate } \\
\text { (24 hrs) }\end{array}$ & 3.55 & 3 & 2.55 & 4.1 & 4.35 & 0.5 & 3.55 & 2.85 & 3.6 & 4.05 \\
\hline
\end{tabular}

$\mathrm{pH}$ :

\begin{tabular}{|c|c|c|c|c|c|c|c|c|c|c|}
\hline & \multicolumn{5}{|c|}{ F } & \multicolumn{5}{|c|}{ Continuous flow } \\
\hline & Base & 12D5 & 12D15 & 14D5 & 14015 & Base & 1205 & 12D15 & 14D5 & 14D15 \\
\hline $\begin{array}{l}\text { Feed/Reactor } \\
\text { (Initial) }\end{array}$ & 7.31 & 7.45 & 7.54 & 7.51 & 7.46 & 7.51 & 7.36 & 7.27 & 7.5 & 7.32 \\
\hline $\begin{array}{l}\text { Permeate } \\
\text { (Initial) }\end{array}$ & 7.22 & 7.44 & 7.49 & 7.36 & 7.43 & 7.4 & 7.24 & 7.19 & 7.36 & 7.3 \\
\hline $\begin{array}{l}\text { Feed } \\
\text { (12 hrs) }\end{array}$ & 7.35 & 7.66 & 7.5 & 7.51 & 7.48 & 7.37 & 7.35 & 7.37 & 7.51 & 7.43 \\
\hline $\begin{array}{l}\text { Reactor } \\
\text { (12 hrs) }\end{array}$ & 7.51 & 7.7 & 7.64 & 7.42 & 7.49 & 7.41 & 7.39 & 7.35 & 7.5 & 7.28 \\
\hline $\begin{array}{l}\text { Permeate } \\
\text { (12 hrs) }\end{array}$ & 7.01 & 7.73 & 7.5 & 7.3 & 7.45 & 7.33 & 7.5 & 7.3 & 7.49 & 7.43 \\
\hline $\begin{array}{l}\text { Feed } \\
\text { (24 hrs) }\end{array}$ & 7.38 & 7.7 & 7.43 & 7.45 & 7.3 & 7.27 & 7.49 & 7.66 & 7.51 & 7.33 \\
\hline $\begin{array}{l}\text { Reactor } \\
\text { ( } 24 \text { hrs) }\end{array}$ & 7.67 & 7.72 & 7.57 & 7.51 & 7.51 & 7.25 & 7.44 & 7.36 & 7.46 & 7.44 \\
\hline $\begin{array}{l}\text { Permeate } \\
\text { (24 hrs) }\end{array}$ & 7.48 & 7.73 & 7.62 & 7.41 & 7.4 & 7.44 & 7.37 & 7.38 & 7.5 & 7.48 \\
\hline
\end{tabular}




\section{APPENDIX L - Main Phase Experiments: $2^{4}$ factorial design}

\section{replicates}

Average of TMP changes observed for experiments 2, 6 and 16 :

\begin{tabular}{|c|c|c|c|}
\cline { 2 - 4 } \multicolumn{1}{c|}{} & Experiment 2 & Experiment 6 & Experiment 16 \\
\hline Rep 1 (mbar) & -52.3 & -51.6 & -14.9 \\
\hline Rep 2 (mbar) & -49.6 & -45.9 & -15.0 \\
\hline $\begin{array}{c}\text { Average } \\
\text { (mbar) }\end{array}$ & -50.9 & -48.7 & -14.9 \\
\hline
\end{tabular}

Experiment 2 Replicates:

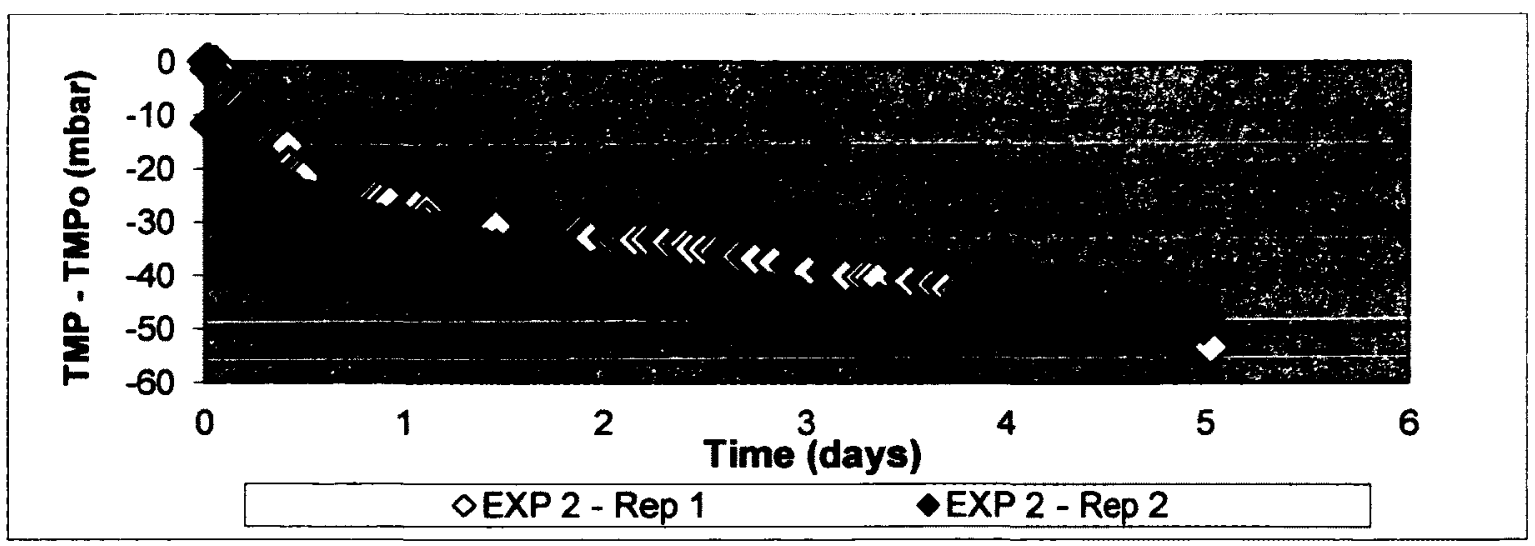


Experiment 6 Replicates:

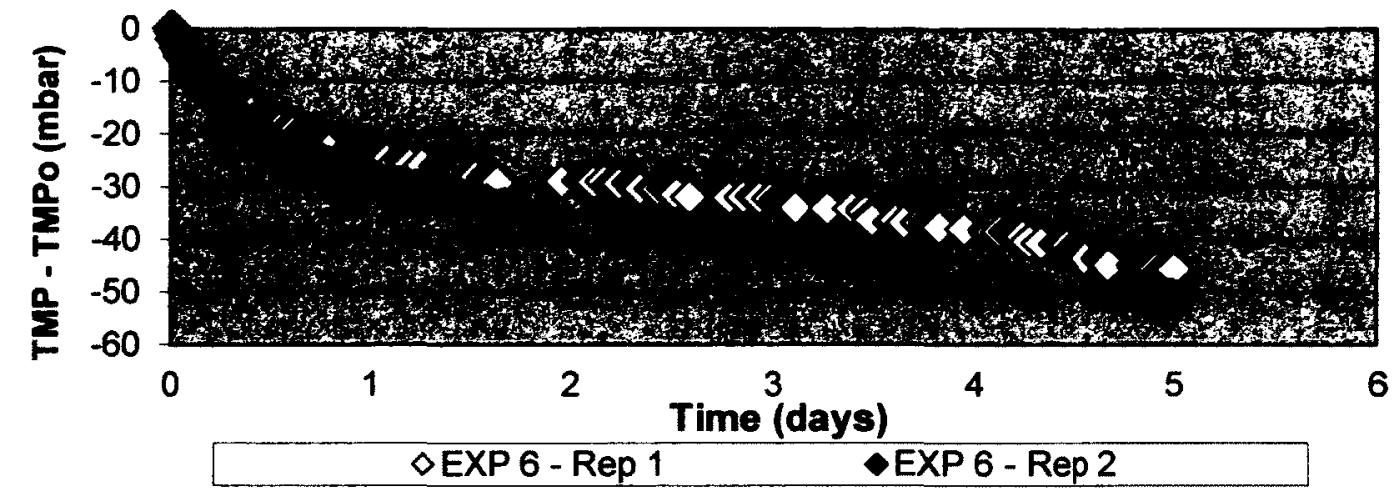

Experiment 10 Replicates:

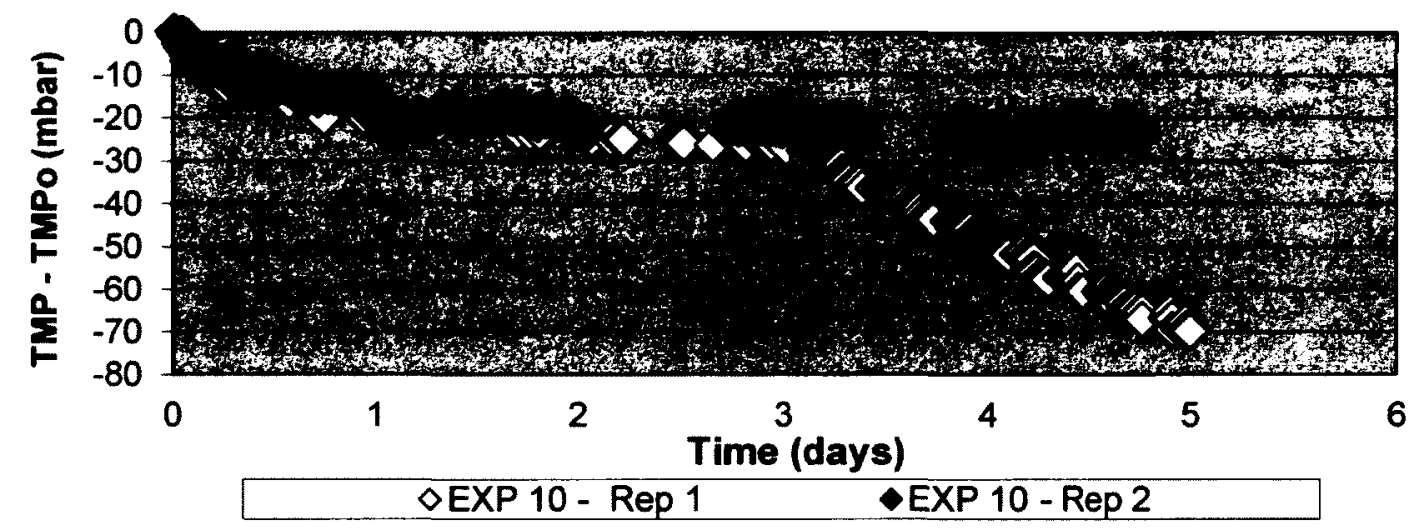

Experiment 16 Replicates:

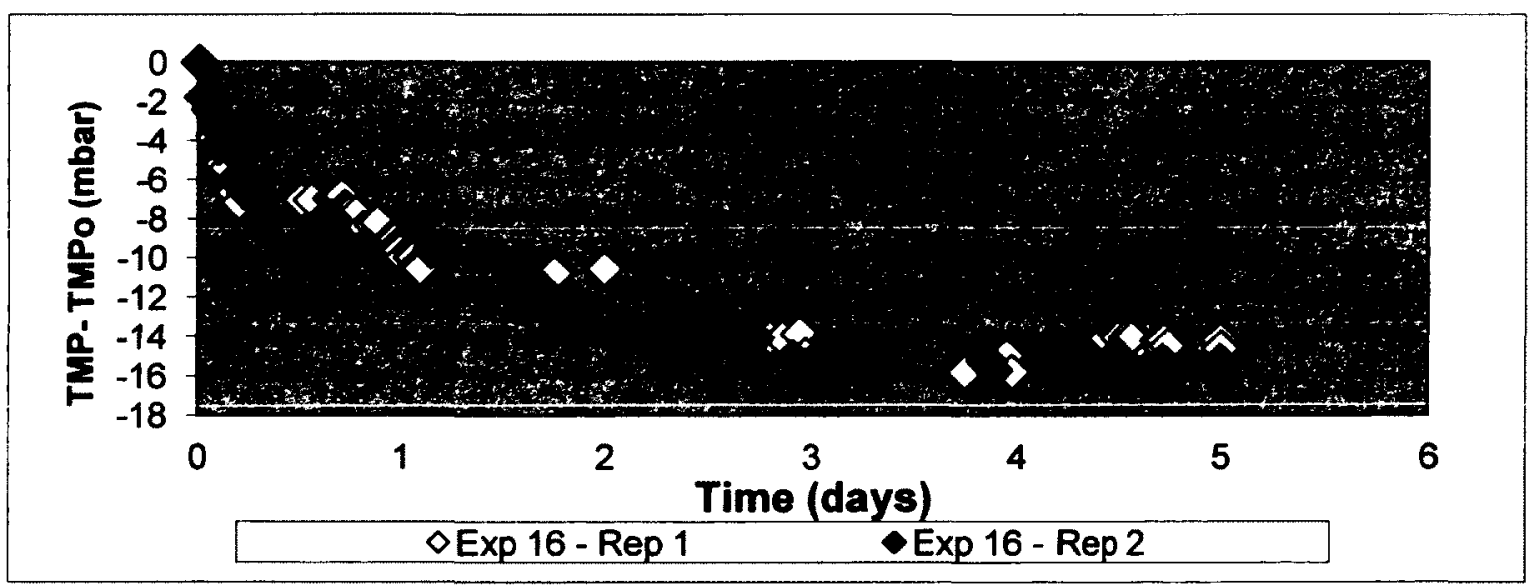




\section{APPENDIX M - 4-way ANOVA model with TMP (Main Phase Experiments)}

\begin{tabular}{|c|c|c|c|c|c|c|c|c|c|c|}
\hline & \multicolumn{3}{|c|}{ Relaxation Duration (5 min) } & \multicolumn{3}{|c|}{ Relaxation Duration (15 min) } & \multicolumn{3}{|c|}{ Average } \\
\hline & & $\begin{array}{l}\text { Backwash } \\
\text { Duration } \\
\text { (10 sec.) }\end{array}$ & $\begin{array}{l}\text { Backwash } \\
\text { Duration } \\
\text { (20 sec.) }\end{array}$ & Avg. & $\begin{array}{l}\text { Backwash } \\
\text { Duration } \\
\text { (10 sec.) }\end{array}$ & $\begin{array}{l}\text { Backwash } \\
\text { Duration } \\
\text { (20 sec.) }\end{array}$ & Avg. & $\begin{array}{l}\text { Backwash } \\
\text { Duration } \\
\text { (10 sec.) }\end{array}$ & $\begin{array}{l}\text { Backwash } \\
\text { Duration } \\
\text { (20 sec.) }\end{array}$ & Av \\
\hline \multirow{5}{*}{$\begin{array}{l}\text { Filtration } \\
\text { Flux (19 } \\
\text { LMH) }\end{array}$} & Air Flow & -7.65 & -5.38 & & -11.45 & -7.79 & & -9.55 & -6.58 & \\
\hline & (5 LPM) & $\mu_{1111}$ & $\mu_{1121}$ & & $\mu_{1112}$ & $\mu_{1122}$ & & $\mu_{111 .}$ & $\mu_{112 .}$ & \\
\hline & Air Flow & -2.76 & -7.17 & & -3.72 & -0.90 & & -3.24 & -4.03 & \\
\hline & & $\mu_{1211}$ & $\mu_{1221}$ & & $\mu_{1212}$ & $\mu_{1222}$ & & $\mu_{121 .}$ & $\mu_{122 .}$ & \\
\hline & Avg. & & & & & & & & & \\
\hline \multirow{4}{*}{$\begin{array}{l}\text { Filtration } \\
\text { Flux (38 } \\
\text { LMH) }\end{array}$} & $\begin{array}{l}\text { Air Flow } \\
\text { (5 LPM) }\end{array}$ & -50.95 & -48.75 & & -23.86 & -15.10 & & -37.40 & -31.92 & \\
\hline & Air Flow & -32.47 & -8.96 & & -11.17 & -14.93 & & -21.82 & -11.95 & \\
\hline & (15 LPM) & $\mu_{2211}$ & $\mu_{2221}$ & & $\mu_{2212}$ & $\mu_{2222}$ & & $\mu_{221 .}$ & $\mu_{222 .}$ & \\
\hline & Avg. & $2 x$ & & & & 8 & & & & \\
\hline \multirow{4}{*}{ Avg } & $\begin{array}{l}\text { Air Flow } \\
\text { (5 LPM) }\end{array}$ & -29.30 & -27.06 & & -17.65 & -11.45 & & -23.48 & -19.25 & \\
\hline & & -17.62 & -8.07 & & -7.45 & -7.91 & & -12.53 & -7.99 & \\
\hline & (15 LPM) & $\mu_{.211}$ & $\mu_{.221}$ & & $\mu_{.212}$ & $\mu_{.222}$ & & $\mu_{.21 .}$ & $\mu .22$. & \\
\hline & Avg. & & & & & 46 & & . & Hen & \\
\hline
\end{tabular}




\section{APPENDIX N - 3-way Interaction Plots - Main Phase}

\section{Experiments}

Interaction between Filtration Flux (FF $\rightarrow$ Hi: 38 LMH, Lo: 19 LMH), Air Flow rate (AF $\rightarrow$ Hi: 15 LPM, Lo: 5 LPM) and Backwash Duration (BD $\rightarrow$ Hi: 20 sec., Lo: $10 \mathrm{sec}$.)
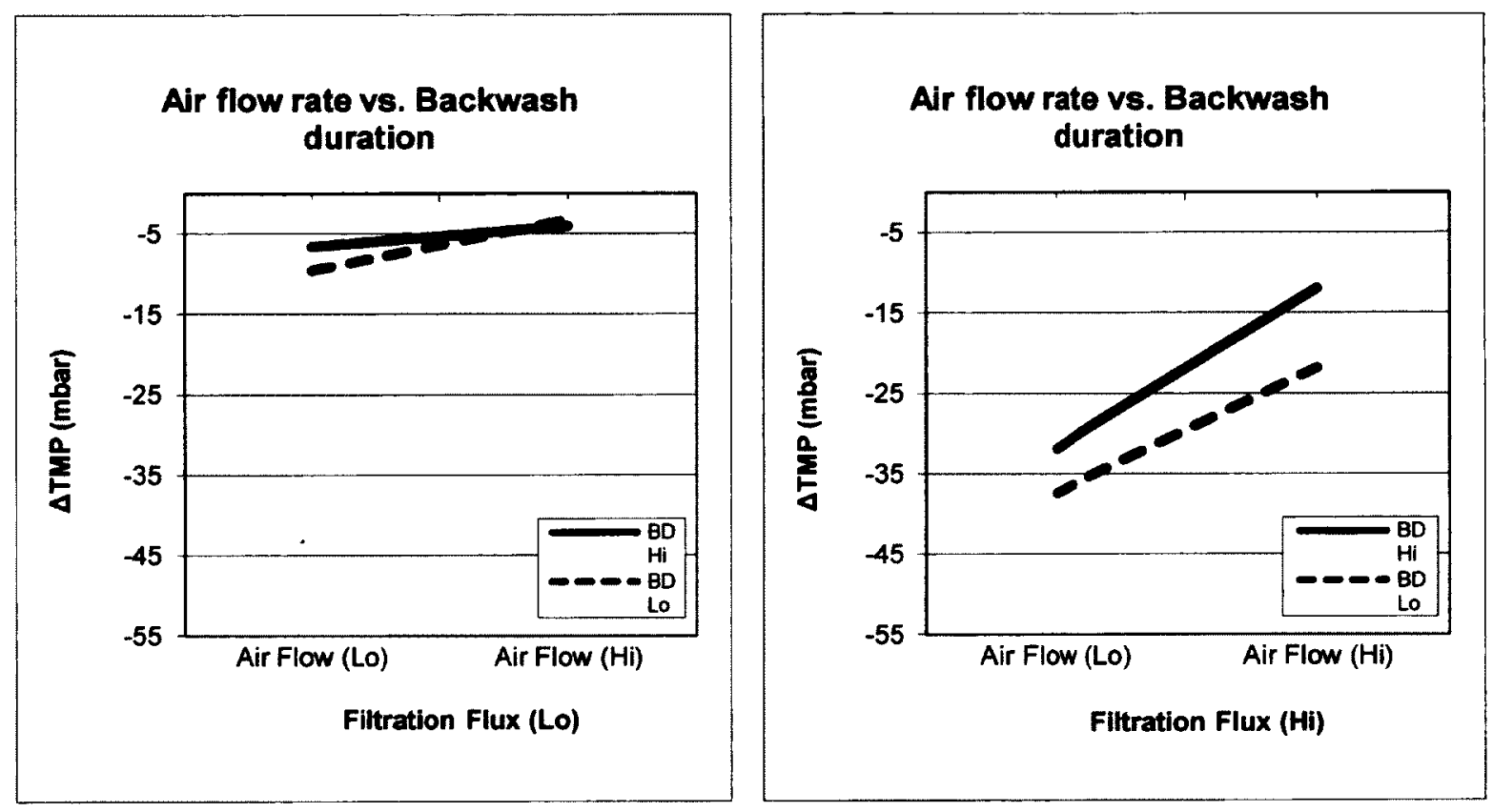

Interaction between Filtration Flux (FF $\rightarrow$ Hi: 38 LMH, Lo: 19 LMH), Air Flow rate (AF $\rightarrow$ Hi: 15 LPM, Lo: 5 LPM) and Relaxation Duration (RD $\rightarrow$ Hi: 15 min., Lo: 5 min.)
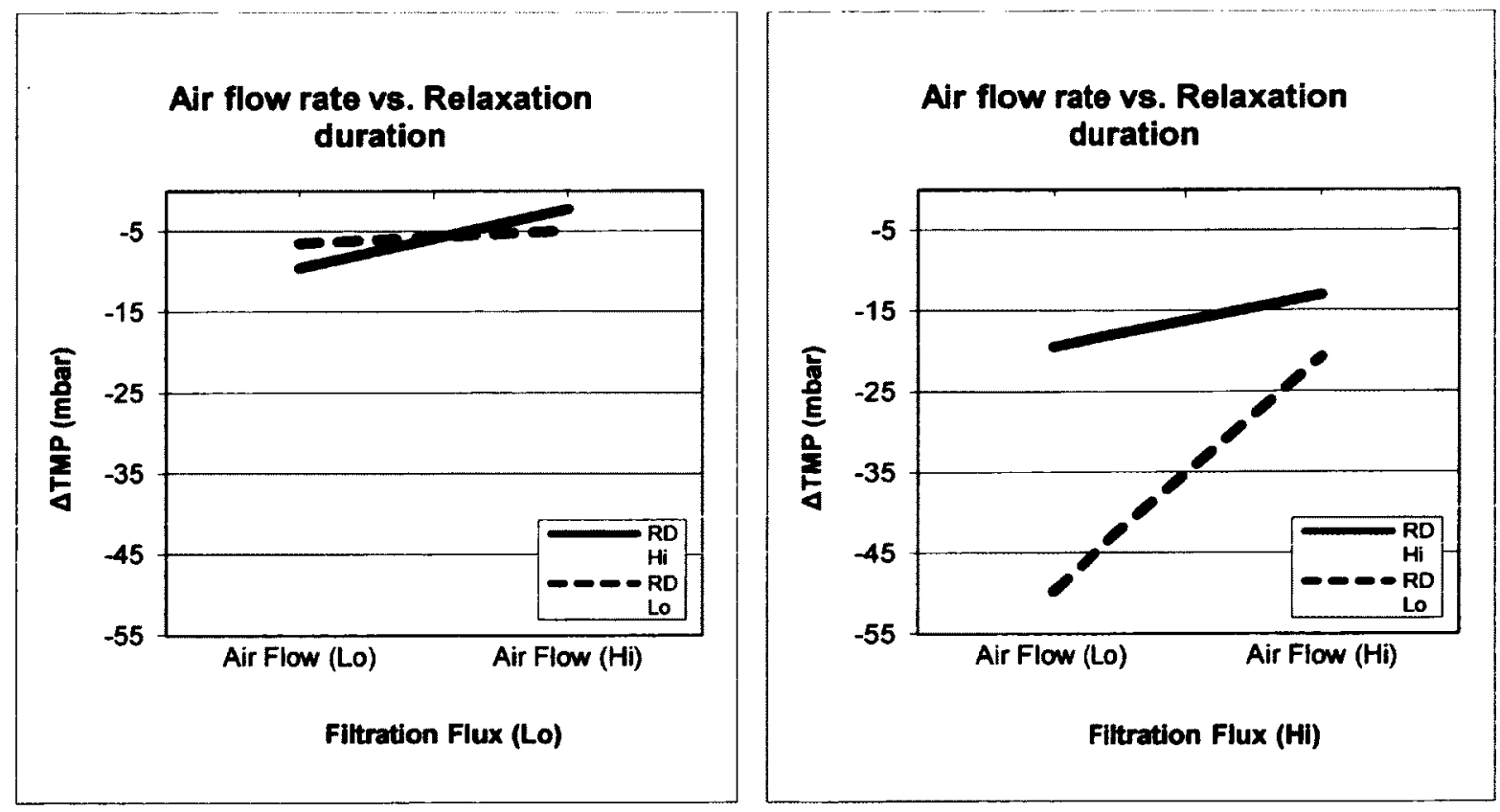
Interaction between Filtration Flux (FF $\rightarrow$ Hi: 38 LMH, Lo: 19 LMH), Backwash Duration (BD $\rightarrow$ Hi: 20 sec., Lo: $10 \mathrm{sec}$.) and Relaxation Duration ( $\mathrm{RD} \rightarrow \mathrm{Hi}: 15 \mathrm{~min}$. , Lo: $5 \mathrm{~min}$.)
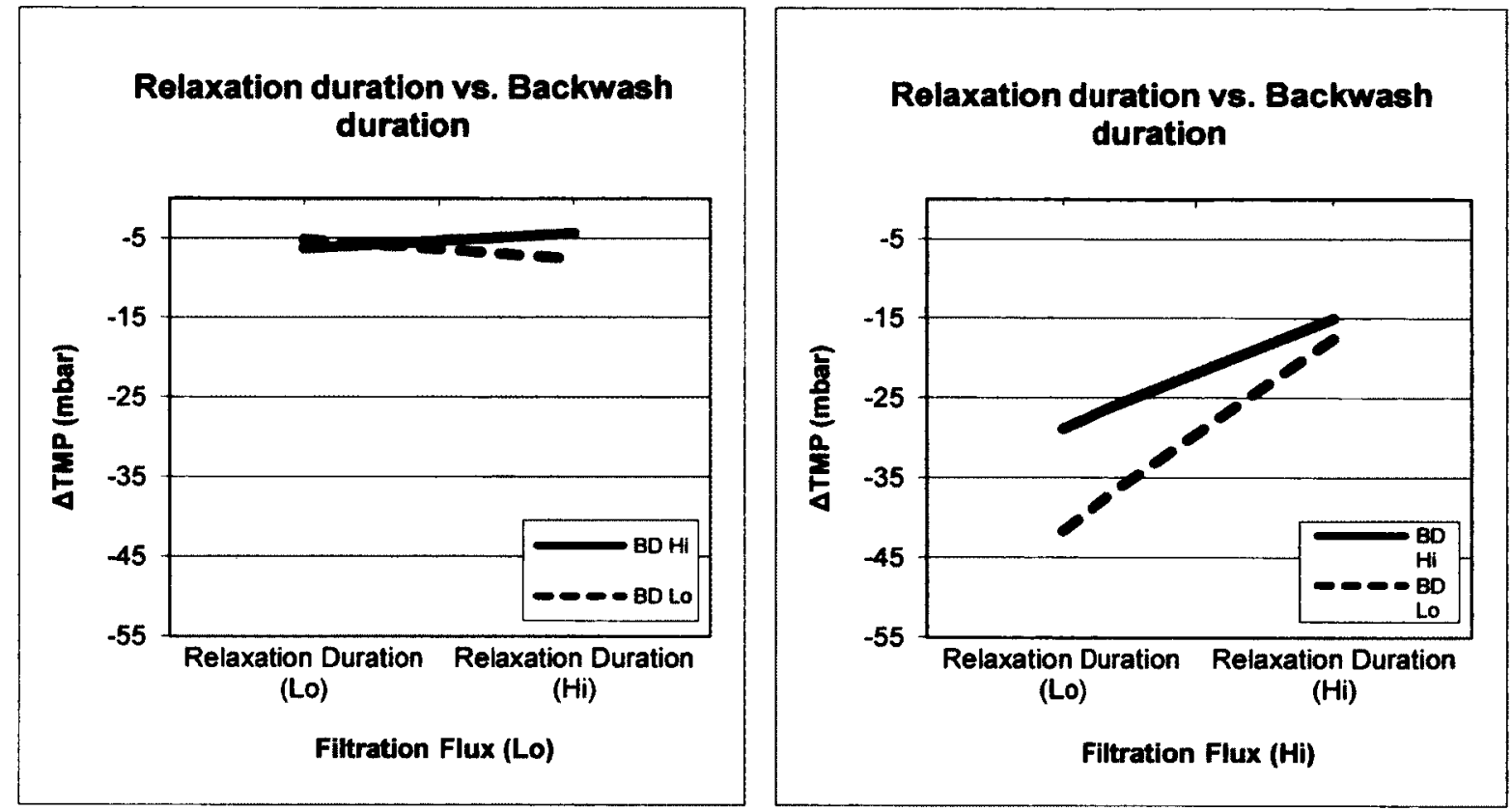

Interaction between Air Flow rate (AF $\rightarrow$ Hi: 15 LPM, Lo: 5 LPM), Backwash Duration (BD $\rightarrow$ Hi: 20 sec., Lo: $10 \mathrm{sec}$.) and Relaxation Duration (RD $\rightarrow$ Hi: $15 \mathrm{~min}$. , Lo: $5 \mathrm{~min}$.)
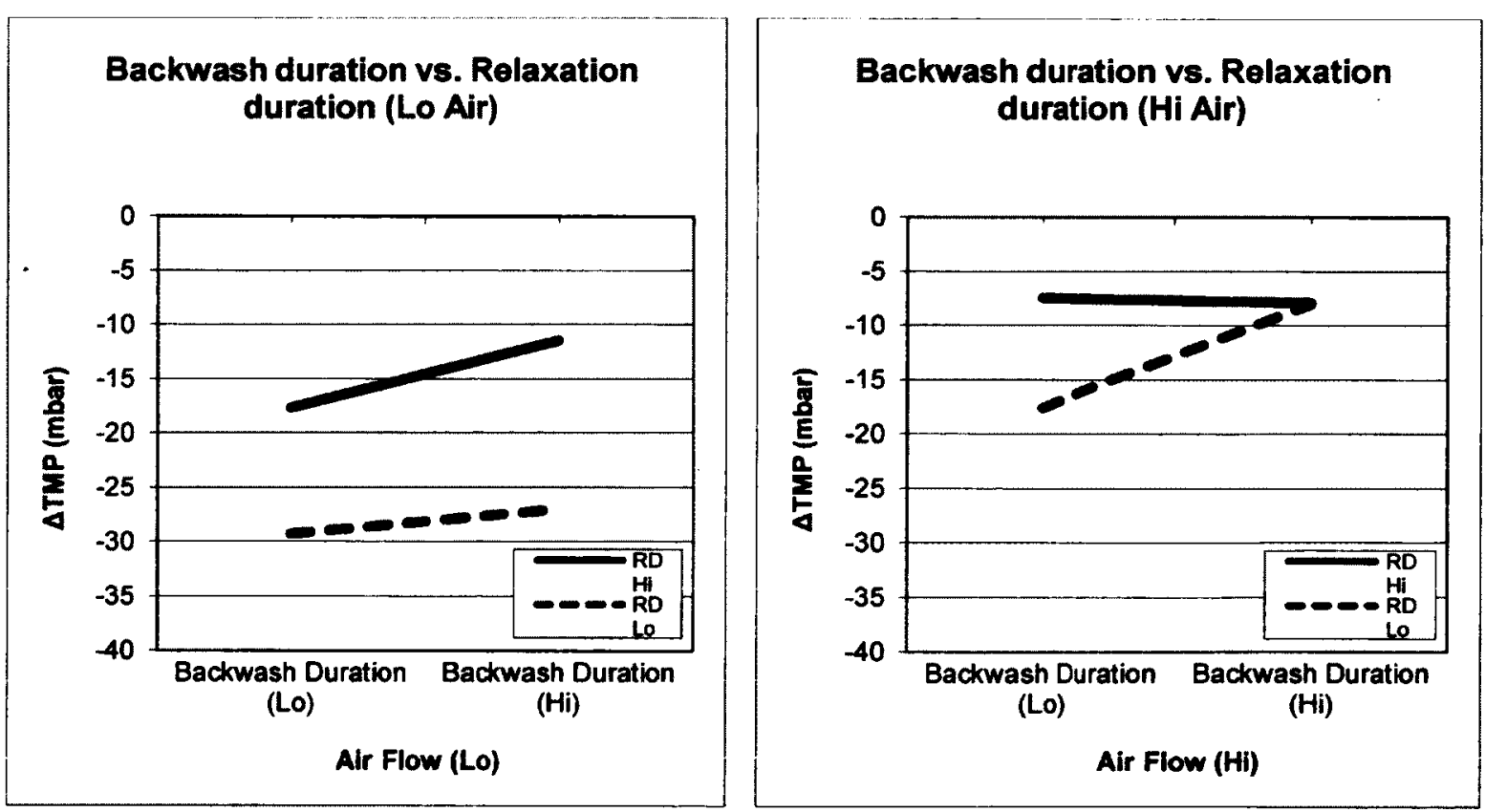


\section{APPENDIX O - Stepwise Linear Regression Output using JMP 9.0 by SAS}

\begin{tabular}{ll}
\hline Stepuries Regreadion Control \\
\hline Stopping & P-value Threshold \\
& Prob to Enter \\
& Prob to Leave \\
& 0.1 \\
\hline Direction: & Mbxed \\
Rules: & Combine
\end{tabular}

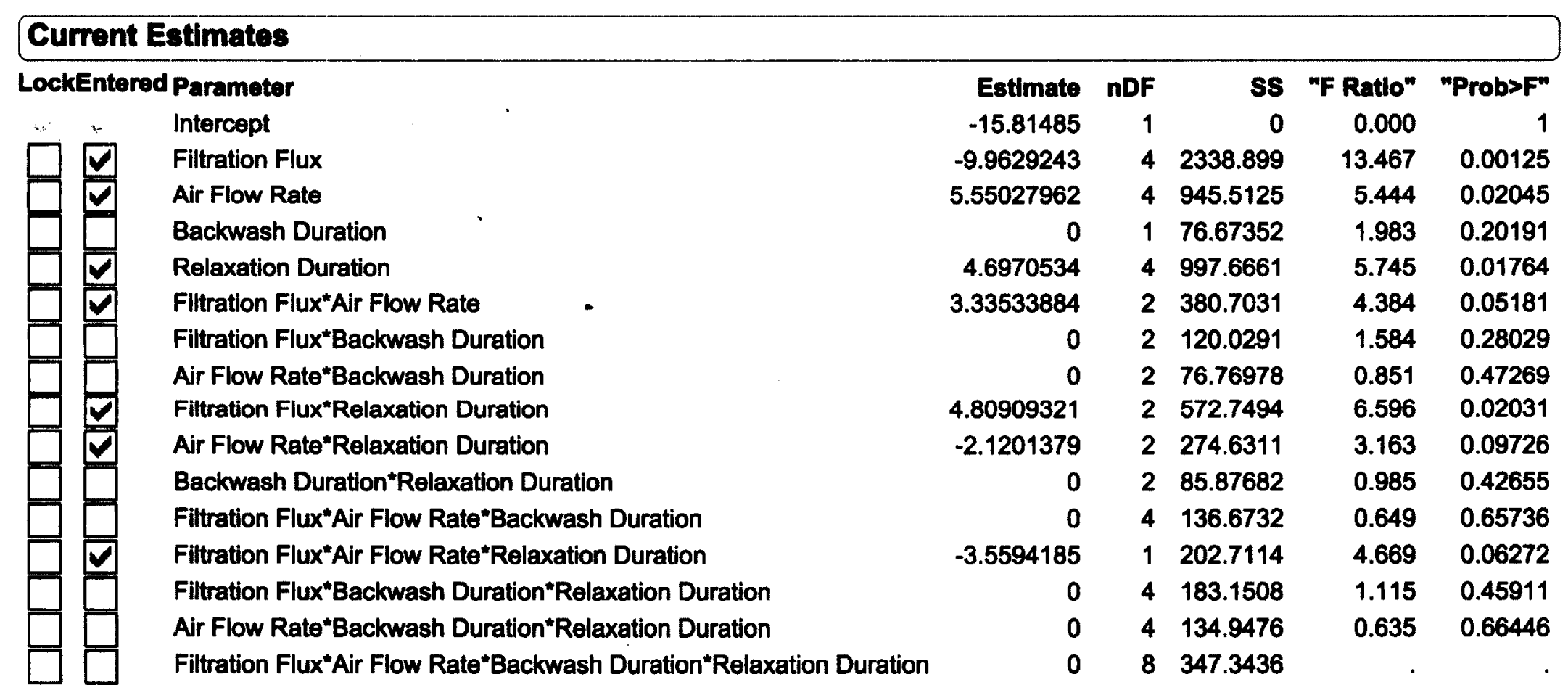




\section{APPENDIX P - Water Quality from Main Phase Low}

\section{Filtration Flux experiments}

\begin{tabular}{|c|c|c|c|}
\hline Sample & $\mathbf{p H}$ & $\begin{array}{l}\text { Turbidity } \\
\text { (NTU) }\end{array}$ & $\underset{(m g / L)}{C O D}$ \\
\hline \multicolumn{4}{|c|}{ 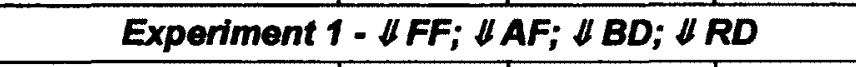 } \\
\hline Feed (Initial) & 7.91 & 30.30 & 18.00 \\
\hline Feed ( 2 days) & 7.91 & 30.23 & 18.00 \\
\hline Feed (5 days) & 7.90 & 26.50 & 16.50 \\
\hline \multicolumn{4}{|c|}{ 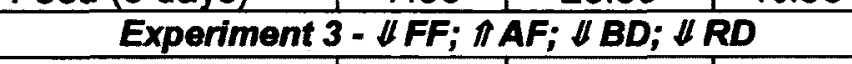 } \\
\hline Feed (Initial) & 7.74 & 30.23 & 18.50 \\
\hline Feed ( 2 days) & 7.73 & 26.00 & 9.50 \\
\hline Feed (5 days) & 7.84 & 27.08 & 13.00 \\
\hline \multicolumn{4}{|c|}{ Experiment 5 - $\Downarrow F F ; \Downarrow A F ; \pi B D ; \Downarrow R D$} \\
\hline Feed (Initial) & 7.96 & 30.67 & 13.00 \\
\hline Feed ( 2 days) & 7.94 & 28.43 & 16.50 \\
\hline Feed (4 days) & 7.90 & 24.90 & 25.00 \\
\hline \multicolumn{4}{|c|}{ Experiment 7 - UFF; $\pi A F ; \pi B D ; \Downarrow R D$} \\
\hline Feed (Initial) & 7.84 & 31.37 & - \\
\hline Feed ( 2 days) & 7.78 & 31.80 & 16.00 \\
\hline Feed ( 5 days) & 7.77 & 30.80 & 15.50 \\
\hline \multicolumn{4}{|c|}{ Experiment 9 - UFF; «AF; UBD; $\pi R D$} \\
\hline Feed (Initial) & 7.75 & 30.97 & 18.00 \\
\hline Feed ( 2 days) & 7.73 & 28.10 & - \\
\hline Feed (5 days) & 7.75 & 26.30 & 16.50 \\
\hline \multicolumn{4}{|c|}{ Experiment 11 - $\Downarrow F F ; \pi A F ; \Downarrow B D ; \pi R D$} \\
\hline Feed (Initial) & 7.73 & 30.93 & 21.00 \\
\hline Feed ( 2 days) & 7.76 & 29.10 & 15.50 \\
\hline Feed ( 5 days) & 7.75 & 28.60 & 12.00 \\
\hline \multicolumn{4}{|c|}{ Experiment 13- $\Downarrow F F ; \Downarrow A F ; \pi B D ; \pi R D$} \\
\hline Feed (Initial) & 7.72 & 30.00 & 17.50 \\
\hline Feed (2 days) & 7.73 & 28.43 & - \\
\hline Feed ( 5 days) & 7.71 & 24.03 & 14.00 \\
\hline \multicolumn{4}{|c|}{ Experiment $15-\| F F ; \Uparrow A F ; \pi B D ; \Uparrow R D$} \\
\hline Feed (Initial) & 7.81 & 33.03 & 21.50 \\
\hline Feed (2 days) & 7.74 & 30.63 & 15.00 \\
\hline Feed (5 days) & 7.79 & 32.30 & 15.00 \\
\hline
\end{tabular}




\begin{tabular}{|c|c|c|c|c|c|c|c|}
\hline Sample & pH & $\begin{array}{c}\text { Turbidity } \\
\text { (NTU) }\end{array}$ & $\begin{array}{c}\text { COD } \\
(\mathrm{mg} / \mathrm{L})\end{array}$ & Sample & pH & $\begin{array}{l}\text { Turbidity } \\
\text { (NTU) }\end{array}$ & $\begin{array}{c}\text { COD } \\
(\mathrm{mg} / \mathrm{L})\end{array}$ \\
\hline \multicolumn{4}{|c|}{ Experiment 1 - $\Downarrow F F ; \| A F ; \Downarrow B D ; \Downarrow R D$} & \multicolumn{4}{|c|}{ Experiment 9 - $\|F F ;\| A F ; \Downarrow B D ; \| R D$} \\
\hline Reactor (Initial) & 7.91 & 30.30 & 18.00 & Reactor (Initial) & 7.75 & 31.0 & 18.0 \\
\hline Reactor (1 day) & 7.85 & 99.17 & - & Reactor (1 day) & 7.71 & 69.5 & - \\
\hline Reactor ( 2 days) & 7.91 & 119.00 & 41.00 & Reactor ( 2 days) & 7.80 & 106.3 & - \\
\hline Reactor ( 3 days) & 7.96 & 86.23 & - & Reactor ( 3 days) & & - & - \\
\hline Reactor (4 days) & 7.93 & 87.35 & - & Reactor (4 days) & 7.72 & 71.2 & $=$ \\
\hline Reactor (5 days) & 7.87 & 84.00 & 31.00 & Reactor (5 days) & 7.77 & 89.6 & 37.5 \\
\hline \multicolumn{4}{|c|}{ Experiment $3-\Downarrow F F ; \rrbracket A F ; \Downarrow B D ; \Downarrow R D$} & \multicolumn{4}{|c|}{ Experiment $11-\|F F ;\| A F ; \Downarrow B D ; \pi R D$} \\
\hline Reactor (Initial) & 7.74 & 30.23 & 18.50 & Reactor (Initial) & 7.73 & 30.93 & 21.00 \\
\hline Reactor (1 day) & 7.73 & 83.17 & - & Reactor (1 day) & 7.71 & 92.50 & - \\
\hline Reactor ( 2 days) & 7.78 & 107.00 & 21.50 & Reactor ( 2 days) & 7.70 & 90.63 & 31.00 \\
\hline Reactor ( 3 days) & 7.80 & 115.00 & - & Reactor ( 3 days) & 7.74 & 92.83 & - \\
\hline Reactor (4 days) & 7.81 & 108.00 & - & Reactor (4 days) & 7.77 & 97.07 & - \\
\hline Reactor (5 days) & 7.89 & 114.50 & 38.00 & Reactor ( 5 days) & 7.77 & 66.07 & 35.50 \\
\hline \multicolumn{4}{|c|}{ Experiment 5 - $\Downarrow F F ; \Downarrow A F ; \pi B D ; \Downarrow R D$} & \multicolumn{4}{|c|}{ Experiment 13 - $\|F F ;\| A F ; \| B D ; \pi R D$} \\
\hline Reactor (Initial) & 7.96 & 30.67 & 13.0 & Reactor (Initial) & 7.72 & 30.00 & 17.50 \\
\hline Reactor (1 day) & 7.91 & 64.13 & - & Reactor (1 day) & 7.70 & 53.30 & - \\
\hline Reactor ( 2 days) & 7.99 & 59.03 & 24.5 & Reactor ( 2 days) & 7.71 & 110.23 & - \\
\hline Reactor ( 3 days) & 7.98 & 50.37 & - & Reactor ( 3 days) & - & - & - \\
\hline Reactor (4 days) & 7.90 & 89.47 & 49.0 & Reactor (4 days) & 7.73 & 45.23 & - \\
\hline Reactor (5 days) & 7.95 & 40.90 & - & Reactor (5 days) & 7.75 & 79.50 & 33.00 \\
\hline \multicolumn{4}{|c|}{ Experiment 7 - $\| F F ; \pi A F ; \pi B D ; \Downarrow R D$} & \multicolumn{4}{|c|}{ Experiment $15-\|F F ; \pi A F ;\| B D ; \pi R D$} \\
\hline Reactor (Initial) & 7.84 & 31.37 & - & Reactor (Initial) & 7.81 & 33.03 & 21.50 \\
\hline Reactor (1 day) & 7.83 & 99.10 & - & Reactor (1 day) & 7.79 & 81.33 & - \\
\hline Reactor ( 2 days) & 7.76 & 105.33 & 36.50 & Reactor (2 days) & 7.74 & 92.83 & 24.50 \\
\hline Reactor ( 3 days) & 7.75 & 137.67 & - & Reactor ( 3 days) & 7.74 & 102.57 & - \\
\hline Reactor (4 days) & 7.79 & 126.00 & - & Reactor (4 days) & 7.76 & 95.27 & - \\
\hline Reactor (5 days) & 7.80 & 122.33 & 70.00 & Reactor (5 days) & 7.79 & 112.33 & 44.00 \\
\hline
\end{tabular}




\begin{tabular}{|c|c|c|c|c|}
\hline Sample & $\begin{array}{c}\text { flow } \\
\text { (mL/min) }\end{array}$ & pH & $\begin{array}{l}\text { Turbidity } \\
\text { (NTU) }\end{array}$ & $\begin{array}{c}\text { COD } \\
(\mathbf{m g} / \mathrm{L})\end{array}$ \\
\hline \multicolumn{5}{|c|}{ Experiment 1 - $\varangle F F ; \Downarrow A F ; \Downarrow B D ; \Downarrow R D$} \\
\hline Permeate (Initial) & 15 & 7.80 & 0.02 & 0.00 \\
\hline Permeate ( 2 days) & 15 & 7.93 & 0.01 & 4.00 \\
\hline Permeate (5 days) & 15 & 7.91 & 0.00 & 3.50 \\
\hline \multicolumn{5}{|c|}{ Experiment $3-\Downarrow F F ; \Uparrow A F ; \Downarrow B D ; \Downarrow R D$} \\
\hline Permeate (Initial) & 15 & 7.88 & 0.02 & 0.50 \\
\hline Permeate ( 2 days) & 15 & 7.88 & 0.00 & 3.00 \\
\hline Permeate ( 5 days) & 15 & 7.88 & 0.01 & 2.00 \\
\hline \multicolumn{5}{|c|}{ Experiment 5 - $\Downarrow F F ; \Downarrow A F ; \Uparrow B D ; \Downarrow R D$} \\
\hline Permeate (Initial) & 15 & 7.80 & 0.03 & 0.5 \\
\hline Permeate ( 2 days) & 15 & 7.96 & 0.02 & 6.0 \\
\hline Permeate ( 4 days) & 15 & 7.94 & 0.03 & 5.0 \\
\hline \multicolumn{5}{|c|}{ Experiment 7- $\Downarrow F F ; \pi A F ; \| B D ; \Downarrow R D$} \\
\hline Permeate (Initial) & 15 & 7.60 & 0.00 & 3.00 \\
\hline Permeate ( 2 days) & 15 & 7.76 & 0.00 & 1.50 \\
\hline Permeate (5 days) & - & 7.76 & 0.00 & 0.50 \\
\hline \multicolumn{5}{|c|}{ Experiment 9 - $\Downarrow F F ; \Downarrow A F ; \Downarrow B D ; \pi R D$} \\
\hline Permeate (Initial) & 15 & 7.57 & 0.00 & 2.0 \\
\hline Permeate ( 2 days) & 15 & 7.58 & 0.01 & - \\
\hline Permeate ( 5 days) & 15 & 7.64 & 0.01 & 1.5 \\
\hline \multicolumn{5}{|c|}{ Experiment 11 - $\Downarrow F F ; \| A F ; \Downarrow B D ; \Uparrow R D$} \\
\hline Permeate (Initial) & 15 & 7.65 & 0.00 & 3.00 \\
\hline Permeate ( 2 days) & 15 & 7.67 & 0.01 & 3.50 \\
\hline Permeate ( 5 days) & 15 & 7.64 & 0.01 & 5.00 \\
\hline \multicolumn{5}{|c|}{ Experiment 13 - $\Downarrow F F ; \Downarrow A F ; \Uparrow B D ; \cap R D$} \\
\hline Permeate (Initial) & 15 & 7.60 & 0.00 & 1.00 \\
\hline Permeate ( 2 days) & 15 & 7.62 & 0.04 & - \\
\hline Permeate (5 days) & 15 & 7.60 & 0.01 & 4.50 \\
\hline \multicolumn{5}{|c|}{ Experiment $15-\|F F ;\| A F ;\|B D ;\| R D$} \\
\hline Permeate (Initial) & 15 & 7.61 & 0.00 & 2.00 \\
\hline Permeate ( 2 days) & 15 & 7.68 & 0.01 & 1.50 \\
\hline Permeate (5 days) & 15 & 7.62 & 0.00 & 0.50 \\
\hline
\end{tabular}




\section{APPENDIX Q - Water Quality from Main Phase High}

\section{Filtration Flux Experiments}

\begin{tabular}{|c|c|c|c|}
\hline Sample & $\mathrm{pH}$ & $\begin{array}{c}\text { Turbidity } \\
\text { (NTU) }\end{array}$ & $\begin{array}{c}\text { COD } \\
(\mathrm{mg} / \mathrm{L})\end{array}$ \\
\hline \multicolumn{4}{|c|}{ Experiment 2 - $\pi F F ; \Downarrow A F ; \Downarrow B D ; \Downarrow R D$} \\
\hline Feed (Initial) & 7.77 & 30.17 & 17.50 \\
\hline Feed ( 2 days) & 7.71 & 25.20 & 16.50 \\
\hline Feed (5 days) & 7.6 & 19.72 & 19.00 \\
\hline \multicolumn{4}{|c|}{ Experiment 4 - IFF; \#AF; UBD; URD } \\
\hline Feed (Initial) & 7.89 & 30.40 & 21.00 \\
\hline Feed ( 2 days) & 7.87 & 30.08 & 16.50 \\
\hline Feed (5 days) & 7.89 & 28.77 & 18.50 \\
\hline \multicolumn{4}{|c|}{ Experiment 6 - $\pi F F ; ~ \Downarrow A F ; \pi B D ; \Downarrow R D$} \\
\hline Feed (Initial) & 7.76 & 30.83 & 21.00 \\
\hline Feed ( 2 days) & 7.74 & 26.23 & 18.00 \\
\hline Feed (5 days) & 7.73 & 18.05 & 14.00 \\
\hline \multicolumn{4}{|c|}{ Experiment 8 - $\pi F F ; \pi A F ; \pi B D ; \varangle R D$} \\
\hline Feed (Initial) & 7.88 & 29.93 & 22.00 \\
\hline Feed ( 2 days) & 7.91 & 30.00 & 18.00 \\
\hline Feed (5 days) & 7.89 & 29.37 & 16.00 \\
\hline \multicolumn{4}{|c|}{ Experiment 10 - $\pi F F ; \Downarrow A F ; \Downarrow B D ; \pi R D$} \\
\hline Feed (Initial) & 7.86 & 31.43 & 17.00 \\
\hline Feed ( 2 days) & - & 25.00 & 11.45 \\
\hline Feed (5 days) & 7.74 & 31.00 & 12.25 \\
\hline \multicolumn{4}{|c|}{ Experiment 12 - $\pi$ FF; $\pi$ AF; $\Downarrow B D ; \pi R D$} \\
\hline Feed (Initial) & 7.89 & 31.70 & 24.00 \\
\hline Feed ( 2 days) & 7.91 & 28.57 & 12.50 \\
\hline Feed ( 5 days) & 7.89 & 26.45 & 11.00 \\
\hline \multicolumn{4}{|c|}{ Experiment 14- - FF; $\Downarrow A F ; \| B D ; \pi R D$} \\
\hline Feed (Initial) & 7.85 & 31.93 & 16.50 \\
\hline Feed ( 2 days) & 7.90 & 24.13 & 13.00 \\
\hline Feed ( 5 days) & 7.89 & 23.40 & 12.50 \\
\hline \multicolumn{4}{|c|}{ Experiment 16 - $\pi$ FF; $\pi$ AF; $\pi$ BD; $\pi R D$} \\
\hline Feed (Initial) & 7.770 & 30.37 & 19.00 \\
\hline Feed ( 2 days) & 7.690 & 28.63 & 12.00 \\
\hline Feed ( 5 days) & 7.74 & 28.88 & 14.00 \\
\hline
\end{tabular}




\begin{tabular}{|c|c|c|c|c|c|c|c|}
\hline Sample & pH & $\begin{array}{l}\text { Turbidity } \\
\text { (NTU) }\end{array}$ & $\begin{array}{c}C O D \\
(\mathrm{mg} / \mathrm{L})\end{array}$ & Sample & pH & $\begin{array}{l}\text { Turbidity } \\
\text { (NTU) }\end{array}$ & $\begin{array}{c}\text { COD } \\
(\mathrm{mg} / \mathrm{L})\end{array}$ \\
\hline \multicolumn{4}{|c|}{ Experiment 2- IFF; $\| A F ; \Downarrow B D ; \Downarrow R D$} & \multicolumn{4}{|c|}{ Experiment 10 - ПFF; $\| A F ; \Downarrow B D ; \Uparrow R D$} \\
\hline Reactor (Initial) & 7.77 & 31.90 & 17.50 & Reactor (Initial) & 7.86 & 31.43 & 17.00 \\
\hline Reactor (1 day) & 7.83 & 73.90 & - & Reactor (1 day) & 7.82 & 91.63 & - \\
\hline Reactor ( 2 days) & 7.79 & 65.98 & 35.00 & Reactor ( 2 days) & - & 90.63 & 37.50 \\
\hline Reactor (3 days) & 7.75 & 68.07 & - & Reactor ( 3 days) & - & - & - \\
\hline Reactor (4 days) & 7.71 & 64.90 & - & Reactor (4 days) & - & - & - \\
\hline Reactor (5 days) & 7.62 & 62.37 & 31.00 & Reactor (5 days) & 7.74 & - & - \\
\hline \multicolumn{4}{|c|}{ Experiment 4 - IFF; $\pi A F ; \Downarrow B D ; \Downarrow R D$} & \multicolumn{4}{|c|}{ Experiment 12- $\pi F F ; \pi A F ; \Downarrow B D ; \Uparrow R D$} \\
\hline Reactor (Initial) & 7.89 & 30.40 & 21.00 & Reactor (Initial) & 7.89 & 31.70 & $24: 00$ \\
\hline Reactor (1 day) & 7.93 & 78.30 & - & Reactor (1 day) & - & - & - \\
\hline Reactor (2 days) & 7.87 & 88.53 & 38.00 & Reactor ( 2 days) & 7.94 & 114.50 & 37.50 \\
\hline Reactor ( 3 days) & 7.9 & 92.80 & - & Reactor ( 3 days) & 7.92 & 84.60 & - \\
\hline Reactor (4 days) & 7.91 & 3 & - & actor (4 days) & 7.95 & 84.20 & - \\
\hline Reactor (5 days) & 7.89 & 81.20 & 36.00 & Reactor ( 5 days) & 7.87 & 70.67 & 14.00 \\
\hline \multicolumn{4}{|c|}{ Experiment 6 - $\Uparrow F F ; \Downarrow A F ; \Uparrow B D ; \sharp R D$} & \multicolumn{4}{|c|}{ 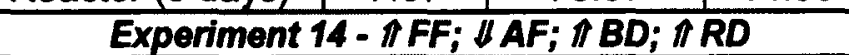 } \\
\hline Reactor (Initial) & 7.76 & 30.83 & 21.00 & Reactor (Initial) & 7.85 & 31.93 & 16.50 \\
\hline Reactor (1 day) & 7.72 & 89.07 & - & Reactor (1 day) & 7.88 & 78.13 & - \\
\hline Reactor (2 days) & 7.73 & 75.53 & 28.50 & Reactor (2 days) & 7.91 & 78.17 & 38.00 \\
\hline Reactor ( 3 days) & 7.70 & 70.97 & - & Reactor ( 3 days) & 7.86 & 74.13 & - \\
\hline Reactor (4 days) & - & - & - & Reactor (4 days) & - & - & - \\
\hline Reactor (5 days) & 7.75 & 86.70 & 31.50 & Reactor ( 5 days) & 7.91 & 56.80 & 26.50 \\
\hline \multicolumn{4}{|c|}{ Experiment 8 - $\pi F F ;\|A F ; \pi B D ;\| R D$} & \multicolumn{4}{|c|}{ Experiment 16 - $\Uparrow F F ; \Uparrow A F ; \pi B D ; \pi R D$} \\
\hline Reactor (Initial) & 7.88 & 29.93 & 22.00 & Reactor (Initial) & 7.77 & 30.37 & 19.00 \\
\hline Reactor (1 day) & 7.88 & 79.33 & - & Reactor (1 day) & 7.77 & 104.33 & - \\
\hline Reactor (2 days) & 7.87 & 89.97 & 39.00 & Reactor ( 2 days) & 7.78 & 104.50 & 38.50 \\
\hline Reactor ( 3 days) & 7.85 & 103.67 & - & Reactor ( 3 days) & 7.72 & 91.70 & - \\
\hline Reactor (4 days) & 7.91 & 90.43 & - & Reactor (4 days) & - & - & - \\
\hline Reactor (5 days) & 7.89 & 104.67 & 45.00 & Reactor (5 days) & 7.76 & 106.00 & 55.50 \\
\hline
\end{tabular}




\begin{tabular}{|c|c|c|c|c|}
\hline Sample & $\begin{array}{c}\text { flow } \\
\text { (mL/min) }\end{array}$ & pH & $\begin{array}{l}\text { Turbidity } \\
\text { (NTU) }\end{array}$ & $\begin{array}{c}\text { COD } \\
(\mathbf{m g} / \mathrm{L})\end{array}$ \\
\hline \multicolumn{5}{|c|}{ Experiment 1 - $/ F F ; \Downarrow A F ; \Downarrow B D ; \Downarrow R D$} \\
\hline Permeate (Initial) & 15 & 7.80 & 0.02 & 0.00 \\
\hline Permeate ( 2 days) & 15 & 7.93 & 0.01 & 4.00 \\
\hline Permeate ( 5 days) & 15 & 7.91 & 0.00 & 3.50 \\
\hline \multicolumn{5}{|c|}{ Experiment 3 - $\|F F ;\| A F ; \Downarrow B D ; \Downarrow R D$} \\
\hline Permeate (Initial) & 15 & 7.88 & 0.02 & 0.50 \\
\hline Permeate ( 2 days) & 15 & 7.88 & 0.00 & 3.00 \\
\hline Permeate ( 5 days) & 15 & 7.88 & 0.01 & 2.00 \\
\hline \multicolumn{5}{|c|}{ Experiment 5- $\| F F ; \Downarrow A F ; \pi B D ; \Downarrow R D$} \\
\hline Permeate (Initial) & 15 & 7.80 & 0.03 & 0.5 \\
\hline Permeate ( 2 days) & 15 & 7.96 & 0.02 & 6.0 \\
\hline Permeate ( 4 days) & 15 & 7.94 & 0.03 & 5.0 \\
\hline \multicolumn{5}{|c|}{ Experiment $7-\Downarrow F F ; \pi A F ; \pi B D ; \Downarrow R D$} \\
\hline Permeate (Initial) & 15 & 7.60 & 0.00 & 3.00 \\
\hline Permeate ( 2 days) & 15 & 7.76 & 0.00 & 1.50 \\
\hline Permeate (5 days) & 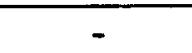 & 7.76 & 0.00 & 0.50 \\
\hline \multicolumn{5}{|c|}{ Experiment 9 - $\Downarrow F F ; \Downarrow A F ; \Downarrow B D ; \pi R D$} \\
\hline Permeate (Initial) & 15 & 7.57 & 0.00 & 2.0 \\
\hline Permeate ( 2 days) & 15 & 7.58 & 0.01 & - \\
\hline Permeate ( 5 days) & 15 & 7.64 & 0.01 & 1.5 \\
\hline \multicolumn{5}{|c|}{ Experiment 11 - $\Downarrow F F ; \Uparrow A F ; \Downarrow B D ; \Uparrow R D$} \\
\hline Permeate (Initial) & 15 & 7.65 & 0.00 & 3.00 \\
\hline Permeate ( 2 days) & 15 & 7.67 & 0.01 & 3.50 \\
\hline Permeate ( 5 days) & 15 & 7.64 & 0.01 & 5.00 \\
\hline \multicolumn{5}{|c|}{ Experiment 13 - $\| F F ; \Downarrow A F ; \pi B D ; \pi R D$} \\
\hline Permeate (Initial) & 15 & 7.60 & 0.00 & 1.00 \\
\hline Permeate ( 2 days) & 15 & 7.62 & 0.04 & - \\
\hline Permeate ( 5 days) & 15 & 7.60 & 0.01 & 4.50 \\
\hline \multicolumn{5}{|c|}{ Experiment $15-\Downarrow F F ; \pi A F ; \pi B D ; \Uparrow R D$} \\
\hline Permeate (Initial) & 15 & 7.61 & 0.00 & 2.00 \\
\hline Permeate ( 2 days) & 15 & 7.68 & 0.01 & 1.50 \\
\hline Permeate ( 5 days) & 15 & 7.62 & 0.00 & 0.50 \\
\hline
\end{tabular}


APPENDIX R - Daily Regression coefficients for 5-day $2^{4}$ factorial Main Phase Experiments

\begin{tabular}{|c|c|c|c|c|c|c|}
\hline Regression Coefficient & Day 0 & Day 1 & Day 2 & Day 3 & Day 4 & Day 5 \\
\hline$\beta_{\mathbf{3}}$ (intercept) & 0 & -9.306 & -11.482 & -13.193 & -14.328 & -15.808 \\
\hline $\boldsymbol{\beta}_{1}$ (FF) & 0 & -5.979 & -7.862 & -8.582 & -9.627 & -9.956 \\
\hline$\beta_{2}$ (AF) & 0 & 2.467 & 3.342 & 3.837 & 4.447 & 5.548 \\
\hline$\beta_{3}$ (BD) & 0 & 1.989 & 3.105 & 2.562 & 2.788 & 2.191 \\
\hline$\beta_{4}$ (RD) & 0 & 2.588 & 2.859 & 3.346 & 3.921 & 4.695 \\
\hline$\beta_{12}$ (FF \& AF) & 0 & 1.381 & 1.980 & 2.329 & 2.943 & 3.333 \\
\hline$\beta_{13}$ (FF \& BD) & 0 & 1.110 & 2.019 & 1.743 & 1.862 & 1.648 \\
\hline$\beta_{14}$ (FF \& RD) & 0 & 2.691 & 3.187 & 3.027 & 4.124 & 4.807 \\
\hline$\beta_{23}$ (AF \& BD) & 0 & -0.933 & -0.782 & -1.075 & -0.422 & 0.080 \\
\hline$\beta_{24}$ (AF \& RD) & 0 & -0.825 & -1.295 & -1.601 & -1.875 & -2.114 \\
\hline$\beta_{34}$ (BD \& RD) & 0 & 0.463 & 0.424 & -0.567 & -0.379 & -0.756 \\
\hline$\beta_{123}$ (FF, AF \& BD) & 0 & 0.670 & 0.838 & 0.605 & 1.168 & 1.019 \\
\hline$\beta_{124}$ (FF, AF \& RD) & 0 & -1.894 & -2.191 & -2.971 & -3.154 & -3.553 \\
\hline$\beta_{134}$ (FF, BD \& RD) & 0 & -0.985 & -1.092 & -1.799 & -1.426 & -1.834 \\
\hline$\beta_{234}$ (AF, BD \& RD) & 0 & -1.079 & -1.566 & -1.739 & -1.737 & -1.747 \\
\hline$\beta_{1234}$ (FF, AF BD \& & 0 & -1.493 & -1.859 & -1.885 & -2.439 & -2.480 \\
\hline RD) & 0 & & &
\end{tabular}

Where FF $\rightarrow$ filtration flux, $\mathrm{AF} \rightarrow$ air flow rate, $\mathrm{BD} \rightarrow$ backwash duration and $\mathrm{RD} \rightarrow$ relaxation duration. 


\section{APPENDIX S - Regression coefficient estimation for 5 day prediction model}

\begin{tabular}{|c|c|c|c|c|c|c|}
\hline \multirow[b]{2}{*}{$\begin{array}{l}\text { Regression } \\
\text { Coefficient }\end{array}$} & \multicolumn{2}{|c|}{ Regression Model 1} & \multicolumn{2}{|c|}{ Regression Model 2} & \multicolumn{2}{|c|}{ Regression Model 3} \\
\hline & Linear & $\mathbf{R}^{2}$ & $\log$ & $\mathbf{R}^{2}$ & $\begin{array}{l}\text { Combination (Linear, } \\
\text { Logarithmic \& } \\
\text { Exponential trends) }\end{array}$ & $\mathbf{R}^{2}$ \\
\hline$\beta$-hato & $-1.6778 x-7.8826$ & 0.9811 & $-3.6281 \operatorname{Ln}(x)-9.1946$ & 0.9948 & $-3.6281 \operatorname{Ln}(x)-9.1946$ & 0.9948 \\
\hline$\beta$-hat & $-1.1663 x-5.0967$ & 0.9596 & $-2.545 \operatorname{Ln}(x)-5.9904$ & 0.9908 & $-2.545 \operatorname{Ln}(x)-5.9904$ & 0.9908 \\
\hline$\beta$-hat 2 & $0.6436 x+1.9144$ & 0.9858 & $1.3833 \operatorname{Ln}(x)+2.4243$ & 0.9876 & $1.3833 \operatorname{Ln}(x)+2.4243$ & 0.9876 \\
\hline$\beta$-hat hat $_{3}$ & $0.1855 x+2.1471$ & 0.2588 & $0.4927 \operatorname{Ln}(x)+2.2194$ & 0.3959 & $0.4927 \operatorname{Ln}(x)+2.2194$ & 0.3959 \\
\hline$\beta$-hat & $0.4488 x+2.0566$ & 0.9768 & $0.9153 \operatorname{Ln}(x)+2.4514$ & 0.881 & $2.2096 e 0.1404 x$ & 0.9897 \\
\hline$\beta$-hat 12 & $0.5035 x+0.8996$ & 0.9896 & $1.0625 \operatorname{Ln}(x)+1.3142$ & 0.9555 & $0.5035 x+0.8996$ & 0.9896 \\
\hline$\beta-$ hat $_{13}$ & $0.198 x+1.1883$ & 0.4109 & $0.5031 \operatorname{Ln}(x)+1.2836$ & 0.5752 & $0.5031 \operatorname{Ln}(x)+1.2836$ & 0.5752 \\
\hline$\beta$-hat 14 & $0.4139 x+2.2225$ & 0.7583 & $0.83 \operatorname{Ln}(x)+2.5978$ & 0.6613 & $2.365600 .1229 x$ & 0.7762 \\
\hline$\beta$-hat h3 & $0.1239 x-1.1129$ & 0.3247 & $0.2247 \operatorname{Ln}(x)-0.9817$ & 0.2317 & $0.1239 x-1.1129$ & 0.3247 \\
\hline$\beta$-hat 24 & $-0.3454 x-0.5354$ & 0.9827 & $-0.7464 \operatorname{Ln}(x)-0.8059$ & 0.9951 & $-0.7464 \operatorname{Ln}(x)-0.8059$ & 0.9951 \\
\hline$\beta$-hat 34 & $-0.3518 x+0.8651$ & 0.7207 & $-0.7451 \operatorname{Ln}(x)+0.5774$ & 0.7008 & $-0.3518 x+0.8651$ & 0.7207 \\
\hline$\beta$-hat ${ }_{123}$ & $0.126 x+0.5053$ & 0.4185 & $0.2378 \operatorname{Ln}(x)+0.6314$ & 0.3231 & $0.126 x+0.5053$ & 0.4185 \\
\hline$\beta$-hat 124 & $-0.4561 x-1.4123$ & 0.9442 & $-0.9623 \operatorname{Ln}(x)-1.7881$ & 0.9113 & $-0.4561 x-1.4123$ & 0.9442 \\
\hline$\beta$-hat ${ }_{134}$ & $-0.2032 x-0.8177$ & 0.5097 & $-0.4594 \operatorname{Ln}(x)-0.9606$ & 0.5651 & $-0.4594 \operatorname{Ln}(x)-0.9606$ & 0.5651 \\
\hline$\beta$-hat ${ }_{234}$ & $-0.2144 x-0.9944$ & 0.7908 & $-0.4981 \ln (x)-1.1346$ & 0.9256 & $-0.4981 \operatorname{Ln}(x)-1.1346$ & 0.9256 \\
\hline$\beta$-hat 1234 & $-0.2863 x-1.2034$ & 0.8982 & $-0.592 \operatorname{Ln}(x)-1.4489$ & 0.8325 & $-0.2863 x-1.2034$ & 0.8982 \\
\hline
\end{tabular}

where $\mathrm{x}=$ time (days); darker text denotes important coefficients 


\section{APPENDIX T - Half Normal Plot for the identification of important effects}

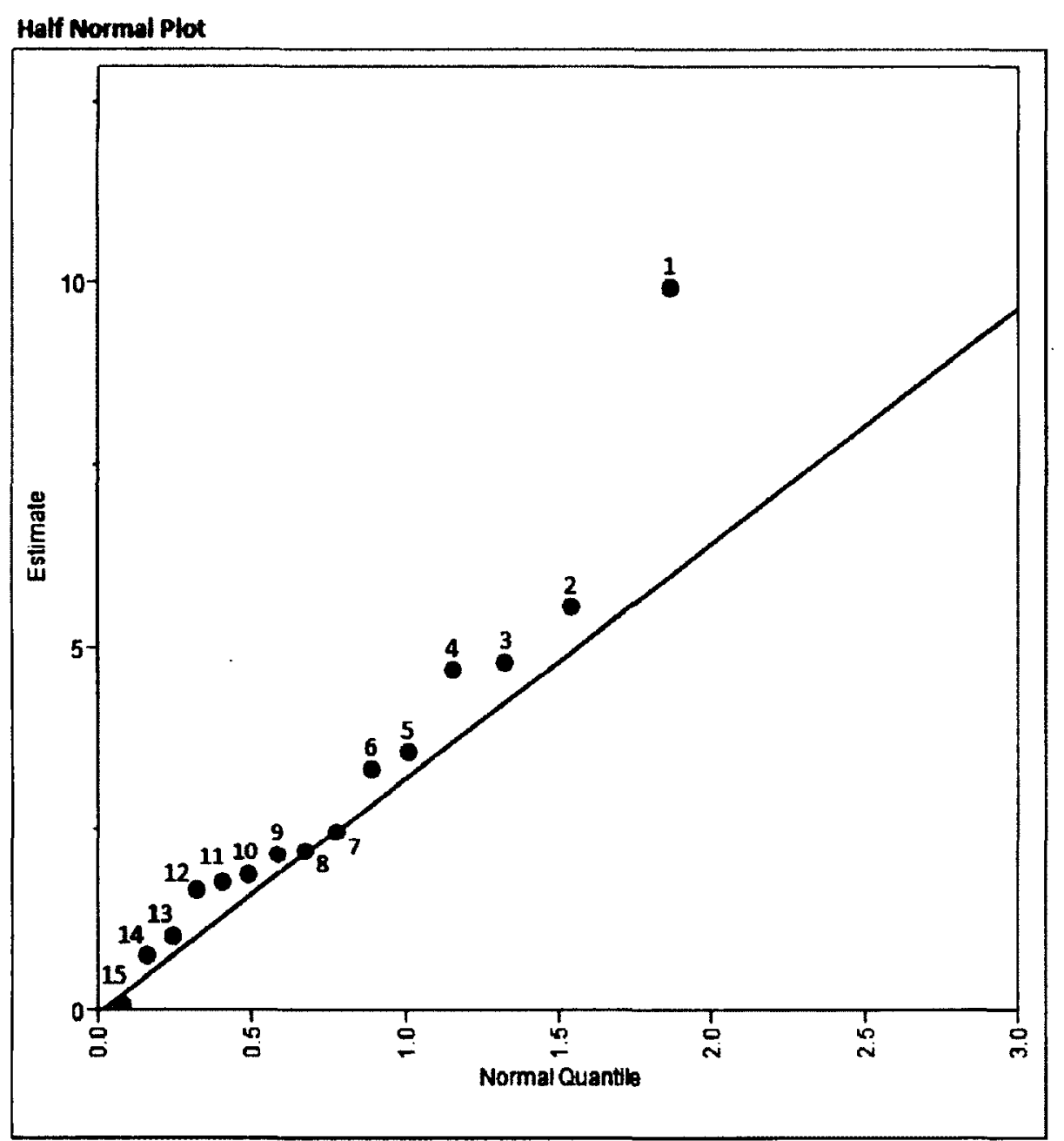

\begin{tabular}{|c|c|c|}
\hline $\begin{array}{l}\text { Rank (based on } \\
\text { marnitude) }\end{array}$ & Main/interaction Effects & Estimate \\
\hline 1 & FF (Filtration Flux) & -9.96 \\
\hline 2 & AF (Air flow rate) & 5.55 \\
\hline 3 & FF"RD & 4.81 \\
\hline 4 & RD /Relaxation duration) & 4.69 \\
\hline 5 & FF"AF"RD & -3.56 \\
\hline 6 & FF=AF & 3.34 \\
\hline 7 & FF"AF"BD & -2.48 \\
\hline 8 & BD (Backwash Duration) & 2.19 \\
\hline 9 & AF*RD & -2.12 \\
\hline 10 & FF"BD*RD & -1.84 \\
\hline 11 & $A F^{*} B D^{*} R D$ & .1 .75 \\
\hline 12 & $F F * B D$ & 1.65 \\
\hline 13 & FF" $^{*} \mathrm{AF}^{*} \mathrm{BD}$ & 1.02 \\
\hline 14 & $B D * R D$ & -0.758 \\
\hline 15 & $A F^{*} B D$ & 0.0776 \\
\hline
\end{tabular}

